INEL-94/01.73

February 1995

An Integrated Systems Approach to Remote Retrieval of Buried Transuranic Waste Using a Telerobotic Transport Vehicle, Innovative End Effector, and Remote Excavator

Ann Marie Smith, LITCO

Phil Rice, LITCO

Randy Peterson, RAHCO International

Reva Hyde, LITCO

Engineering

Laboratory

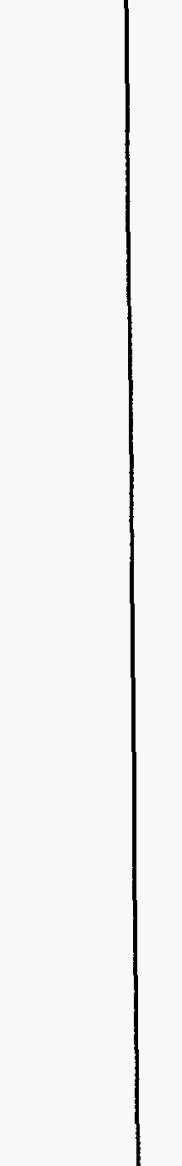

National

Idaho
tional
tory 



\section{DISCLAIMER}

Portions of this document may be illegible in electronic image products. Images are produced from the best available original document. 


\title{
An Integrated Systems Approach to Remote Retrieval of Buried Transuranic Waste Using a Telerobotic Transport Vehicle, Innovative End Effector, and Remote Excavator
}

\author{
Ann Marie Smith, LITCO \\ Phil Rice, LITCO \\ Randy Peterson, RAHCO International \\ Reva Hyde, LITCO
}

Published February 1995

Idaho National Engineering Laboratory Lockheed Idaho Technologies Company Idaho Falls, Idaho 83415

Prepared for the

U.S. Department of Energy

Assistant Secretary for Environmental Management

Under DOE Idaho Operations Office

Contract No. DE-AC07-94ID13223

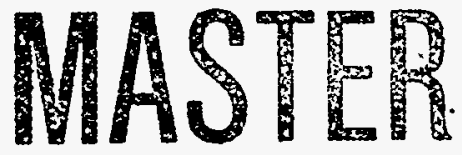




\section{An Integrated Systems Approach to Remote Retrieval of Buried Transuranic Waste Using a Telerobotic Transport Vehicle, Innovative End Effector, and Remote Excavator}

INEL-94/0173 .

Prepared by

Q. M. Smith)

A. M. Smith, Engineering Specialist

Mechanical, Civil, and Industrial Engineering

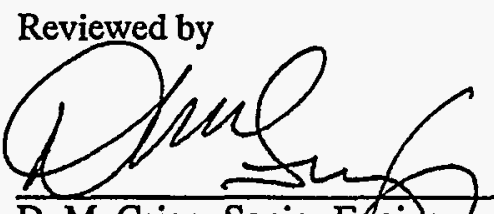

D. M. Grigg, Senior Efginee,

Environmental Restoration Planning and Integration

Approved by

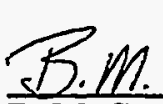

B. M. Gardner, Project Manager

Chemical and Environmental Engineering

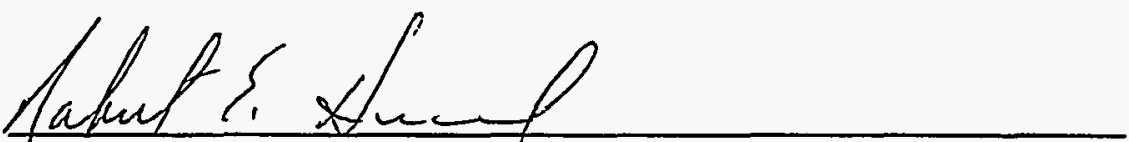

R. E. Heard, Program Manager

Buried Waste Integrated Demonstration

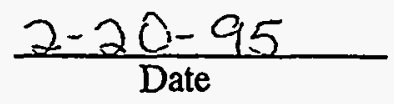

$\frac{2-20-95}{\text { Date }}$

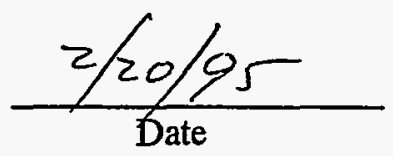




\section{ABSTRACT}

Between 1952 and 1970, over two million cubic feet of transuranic mixed waste was buried in shallow pits and trenches in the Subsurface Disposal Area at the Idaho National Engineering Laboratory Radioactive Waste Management Complex. Commingled with this two million cubic feet of waste is up to 10 million cubic feet of fill soil. The pits and trenches were constructed similarly to municipal landfills with both stacked and random dump waste forms such as barrels and boxes. The main contaminants are micron-sized particles of plutonium and americium oxides, chlorides, and hydroxides. Retrieval, treatment, and disposal is one of the options being considered for the waste.

This report describes the results of a field demonstration conducted to evaluate technologies for excavating, and transporting buried transuranic wastes at the INEL, and other hazardous or radioactive waste sites throughout the U.S. Department of Energy complex.

The full-scale demonstration, conduced at RAHCO International's facilities in Spokane, Washington, in the summer of 1994, evaluated equipment performance and techniques for digging, dumping, and transporting buried waste. Three technologies were evaluated in the demonstration: an Innovative End Effector for dust free dumping, a Telerobotic Transport Vehicle to convey retrieved waste from the digface, and a Remote Operated Excavator to deploy the Innovative End Effector and perform waste retrieval operations. Data were gathered and analyzed to evaluate retrieval performance parameters such as retrieval rates, transportation rates, human factors, and the equipment's capability to control contamination spread. 


\section{SUMMARY}

The development, demonstration, testing, and evaluation of a system for retrieval, conveyance, and dust free dumping of buried waste was funded by the U.S. Department of Energy's Office of Technology Development through the Buried Waste Integrated Demonstration (BWID) Program. The concept and design of the system was developed for the BWID Program by RAHCO International in conjunction with SPAR Aerospace Ltd., and RSI Research Ltd. In the summer of 1994, a full-scale demonstration was conducted at RAHCO's facilities in Spokane, Washington, to evaluate equipment performance and techniques for digging, dumping, and transporting buried waste.

The objective of the demonstration was to explore the effectiveness of methods and equipment to remotely retrieve and transport buried waste away from the digface and control dust generation and contamination spread while dumping retrieved waste. Three technologies were evaluated in the demonstration: (1) an Innovative End Effector (IEE) for dust free dumping, (2) a Telerobotic Transport Vehicle (TTV) to convey retrieved waste from the digface, and (3) a Remote Operated Excavator (REMEX) to deploy the IEE and perform waste retrieval operations. The TTV and IEE were developed and demonstrated by RAHCO with SPAR, and RSI. The REMEX is an existing remote-operated robotic excavator provided by SPAR and modified to accept the IEE. These systems were developed or modified in close coordination with one another to ensure a systems design approach that maximized the effectiveness of the combined technologies.

The IEE is a full-size excavator end effector consisting of a thumb, shovel assembly, and a detachable bucket or integrated transfer module (ITM). The attachable/detachable ITM acts as a detachable loading, handling, and conveyance bucket when digging, handling, and conveying 55-gal drums, dirt, and small debris.

The TTV is a remote-operated, robotic-tracked vehicle capable of maneuvering on rugged terrain and in soft soils as may be expected in buried waste retrieval areas. The guidance and control systems consist of the TTV control system, operator control station, and three radio frequency links between the TTV control system and operator control station. The TTV control system has three separate microprocessor controllers to provide dedicated track control, waste container operation/control, and selected control of the forward and rear-mounted vehicle cameras with pan and tilt units. The TTV also features a waste transport container (WTC) capable of transporting retrieved waste in the form of filled ITMs, $4 \times 4 \times 8$-ft boxes, and many other expected, irregularly shaped, large waste items.

The REMEX is a 40,000-lb class Hitachi EX-200LC standard excavator that has been modified for remote operations by SPAR and RSI. The modifications include the use of coordinated control technology to control the remote operation of the excavator. A four degree of freedom joystick, mounted on the arm of an operator's chair, controls the excavator digging functions. The primary mode of operation is from a remote control operating station via a telemetry cable. The excavator was modified to a front shovel configuration to accept the IEE and thumb for this demonstration.

The demonstration was divided into two phases. Phase I consisted of a full-scale test to dig and dump dry, INEL soils mixed with simulated waste to assess the ability of the innovative end effector to control dust generation and the potential spread of contamination during dumping operations. Phase I of the demonstration was performed inside a building where conditions could be sufficiently controlled to 
simulate a retrieval environment. Dust and rare earth tracer spread data were collected using air monitors. The demonstration included both digging and dumping operations; however, the primary objective was to test the ability of the system to reduce the generation and spread of dust during the dumping operation. Phase II consisted of a full-scale retrieval of simulated buried waste using the TTV, IEE, and REMEX. The purpose of Phase II was to assess the ability of the end effector, waste conveyance system, and REMEX to effectively handle, transfer, and transport the waste from the digface as an integrated system.

Results from the Phase I tests show the IEE to be an effective and reliable system for retrieving and transporting buried waste through the excavating process. The IEE is rugged and well suited for digging heterogeneous buried waste. Retrieval rates of $80 \mathrm{yd}^{3} /$ day or more can easily be achieved. The IEE was effective at handling the various waste forms present in the demonstration. In particular, the flexibility of being able to release the ITM and work with the IEE thumb to pick up objects and place them into the ITM or to move items around on the digface added to the ability of the system to handle a large variety of waste forms. The IEE and thumb were found to be dexterous and capable of handling waste forms ranging from 55-gal drums to loose paper and soil. The overall system integration between the REMEX, IEE, ITM, and WTC was excellent and added to the smooth and successful completion of the Phase I demonstration.

The process of using the IEE resulted in dust spread that was two orders of magnitude below the test goal. The transfer of the ITM from the excavator to the transfer point was accomplished with virtually no visible dust or tracer spread as expected. The Phase I demonstration successfully proved that the concept of eliminating the dumping at the digface is a viable, immensely practical approach to limiting potential contamination spread during buried waste retrieval. Controlling the dust generation at the source of digging combined with the innovative end effector's inherently dust free dumping operation should result in substantially greater than $98 \%$ reduction in dust spread over cases without regard for contamination control.

The Phase II test combined the REMEX, TTV, and ITM system to demonstrate the integrated systems approach of the design in a full-scale retrieval. The TTV and IEE were designed as a system for enhanced performance. The spring bed on the TTV assisted with easy alignment and transfer of empty ITMs from the TTV to the REMEX, while the tapered recess in the WTC assisted with alignment during depositing full ITMs. The systems approach used provided for smooth and easy integration between the TTV, IEE, and REMEX.

The throughput data show that the system had a calculated production rate of $45.42 \mathrm{yd}^{3} / \mathrm{day}$ during the demonstration. With only slight modifications, a single TTV/REMEX system with multiple ITMs would easily achieve 80 to $130 \mathrm{yd}^{3} /$ day and multiple TTVs could greatly increase throughput rates. Lack of sufficient horsepower on the TTV was the single factor that most limited throughput during this demonstration; this is also one of the easiest items to correct.

The capability of the system to handle the various waste forms is excellent. The IEE and TTV integrated well and there was virtually no spillage when loading and transporting the waste. Operator procedures and approaches such as detaching the ITM to see the digface and placing objects directly into the ITM were facilitated by the system's flexibility and enhanced the IEE's ability to handle waste forms and reduce dust generation during retrieval operations. The IEE and thumb were extremely effective in handling and transporting the large objects from the digface. This operation was completed without any drops or slippage of the large objects. 
Piloting the TTV was impaired by the lack of adequate horsepower and uneven power application to the tracks. Work arounds through equipment modifications and modified piloting methods were used to successfully complete the demonstration. The primary effect of this was that the vehicle speed had to be reduced significantly to maintain sufficient horsepower output to easily maneuver. Other than persistent minor engine problems, TTV reliability was excellent.

The TTV control system functioned well throughout the tests. Telemetry tests showed that the control system range easily met the 500 -ft goal. The wireless video worked well and provided a clear video signal to the TTV operator, although there was some interference from the antenna mast in specific orientations and range was limited to less than $250 \mathrm{ft}$. The radio frequency emergency stop malfunctioned and repeatedly shut the TTV engine down, so one redundant relay was bypassed to continue the demonstration. Bypassing this relay did not compromise safety. Ultrasonic sensors mounted on the TTV provided information to the TTV operator to dock at the targets. Some crosstalk between two ultrasonic sensors gave erroneous readings to the operator. These readings were far enough from the actual readings so that the two could be easily distinguished. In addition, the ultrasonic sensors sometimes identified the uneven terrain as an obstacle-a problem that can easily be corrected through sensor placement and/or settings.

The data collected for the maneuverability tests show that the TTV can repeat its placement to the targets within the specified 1 radial foot $77 \%$ of the time. Crosstalk between ultrasonic sensors contributed to the less than expected results. A minor software change would easily eliminate sensor crosstalk, but was not readily field implementable. The TTV had no significant problems negotiating the terrain or the 10 degree sloped hill after the horsepower output was tuned.

The equipment used for this demonstration was designed to investigate concepts for retrieval, dust free dumping, and conveyance of buried waste. Prototype hardware and low cost components were adapted to facilitate an inexpensive, short schedule, proof-of-concept demonstration. The hardware concept and design approach supports a transuranic (TRU) environment retrieval, however due to cost and schedule constraints, no attempt was made to provide fully industrialized equipment. There were no aspects of the equipment design that would make it difficult or prevent it from being upgraded to operate in a TRU buried waste retrieval environment.

The IEE proved that dust free dumping is an efficient, effective means for reducing contamination spread during retrieval operations. The IEE design can provide the same capability as standard end effectors for retrieval operations without the inherent problem of contamination caused by a dusty dumping process. Following transport to a treatment facility, the ITMs can be dumped in a glovebox environment where dust controls and safety systems are more easily implemented. The integrated systems design approach for the development of the REMEX, IEE, and TTV provides an effective solution for remote retrieval and conveyance of buried waste. This demonstration successfully showed that buried waste retrieval can be performed remotely at low cost, at reasonable throughput rates, and with greatly improved safety for the worker and environment.

The data obtained in this demonstration will eventually be used by Environmental Restoration during the feasibility study for parts of the INEL Radioactive Waste Management Complex (RWMC) and, possibly, by additional programs in other locations. Environmental Restoration will also use the data to project additional technology needs for BWID out-year planning. 



\section{ACKNOWLEDGMENTS}

The successful completion of this project was due to the significant contributions and efforts of the entire project team. The authors would like to express their sincere appreciation to those who contributed to the project and to the completion of this report, including Dave Lokhorst and Wayne Chin of RSI Research, Ltd.; Tom Tucker and Joe Sheppard of SPAR Aerospace, Ltd.; and Bill Bauer, Gerry Harris, Guy Loomis, Greg Wachs, Frank Bruneel, Jill White, and Penny Simon of Lockheed Idaho Technologies Company.

In addition, a special thanks go out to these members of the project team for their significant contributions to the project and demonstration: Tom Crocker, Daryl G. Girsberger, Jim Challinor, Pat Burnham, Karen Nishiyama, Rob Larrison, and Jeff Vining of RAHCO International; Derek Foster of RSI Research Ltd.; Clive Holloway of SPAR Aerospace, Ltd.; Randy Kenworthy and Tim Johnston of Lon Gibbey Productions; and Brad Gardner, Brad Griebenow, Lewis Rounds, and John Morrison of Lockheed Idaho Technologies Company. 



\section{CONTENTS}

ABSTRACT

iii

SUMMARY

$\mathbf{v}$

ACKNOWLEDGMENTS

ix

ACRONYMS.

xvii

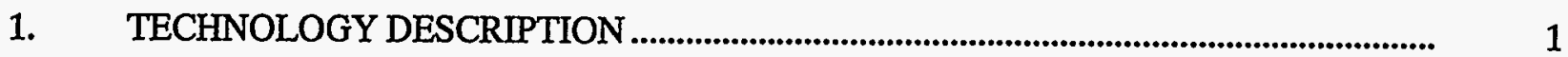

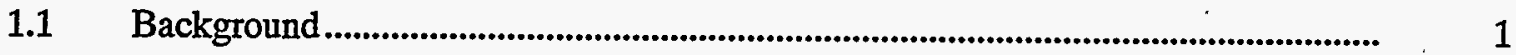

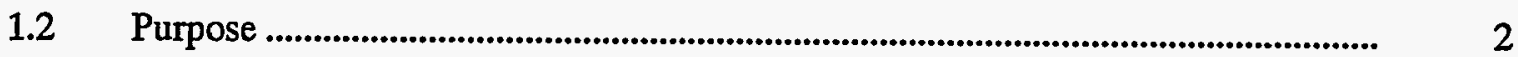

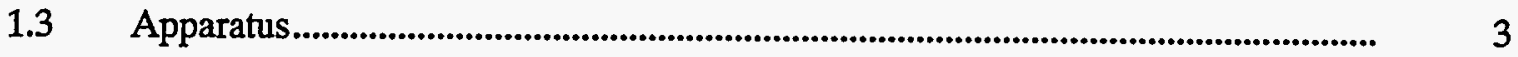

1.3.1 Innovative End Effector................................................................................. 3

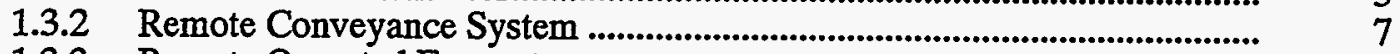

1.3.3 Remote Operated Excavator ......................................................................... 13

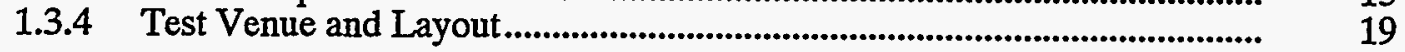

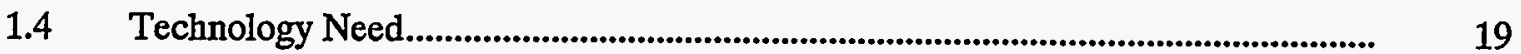

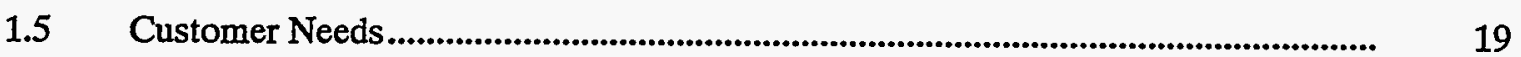

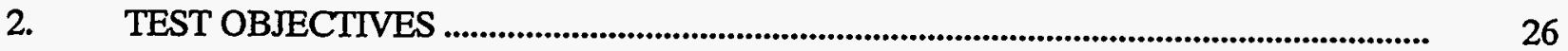

$2.1 \quad$ Phase I Demonstration Test and Data Quality Objectives ......................................... 26

2.1.1 Dust Measurements ..................................................................................... 26

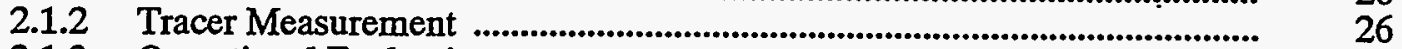

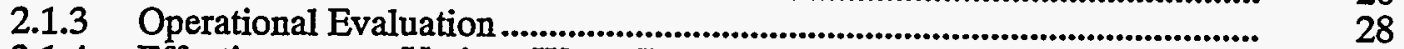

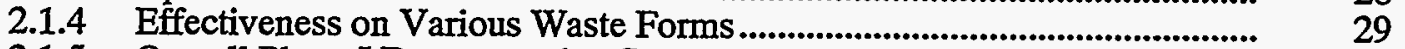

2.1.5 Overall Phase I Demonstration Success .......................................................... $\quad 30$

2.2 Phase II Demonstration Test and Data Quality Objectives ....................................... 30

2.2.1 Throughput .................................................................................................... 30

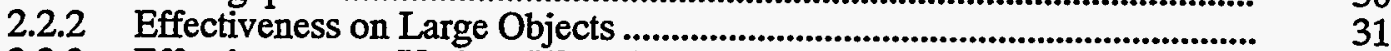

2.2.3 Effectiveness on Various Waste Forms............................................................... 31

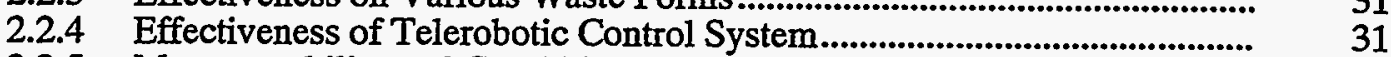

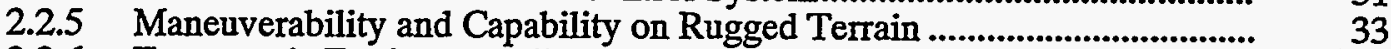

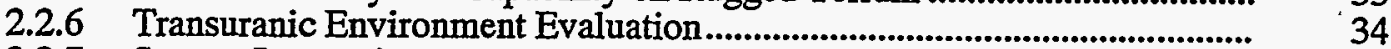

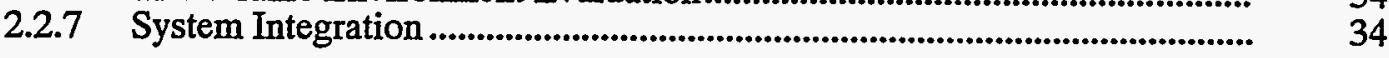

3. EXPERIMENTAL DESIGN AND PROCEDURES ............................................................ 35

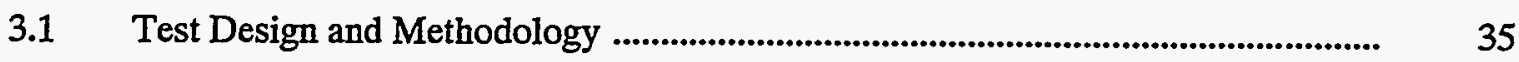

3.1.1 Phase I Demonstration-Dust and Contamination Spread ........................ 35

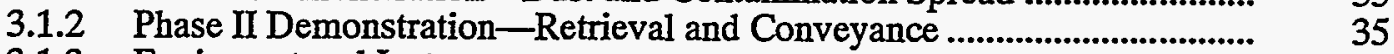

3.1.3 Equipment and Instruments .......................................................................... $\quad 39$

3.2 Deviations from Test Plan .................................................................................. 40

3.3 Deviations from Test Procedure ...................................................................... 41 


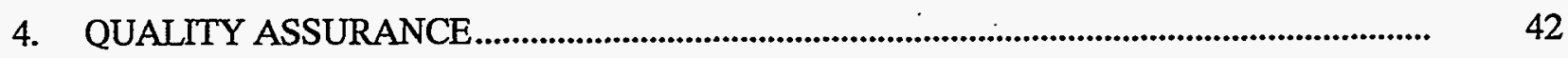

4.1 Quality Control Methods and Procedures ............................................................ 42

4.1.1 Measures Taken ........................................................................................... 42

4.1.2 Effectiveness..................................................................................... 43

4.2 Precision and Accuracy of Measured Data................................................................ 44

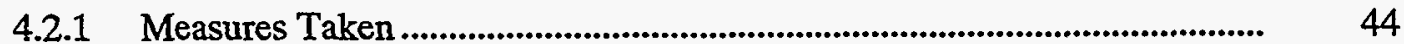

4.2.2 Results and Effectiveness .................................................................... 44

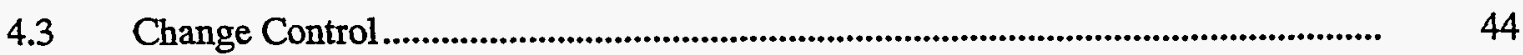

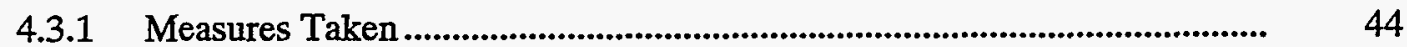

4.3.2 Results and Effectiveness .............................................................................. 45

Internal Audits .................................................................................................. 45

4.4.1 Measures Taken ..................................................................................... 45

4.4.2 Results and Effectiveness ........................................................................... 45

4.5 Subcontractor Quality Assurance Measures............................................................... 45

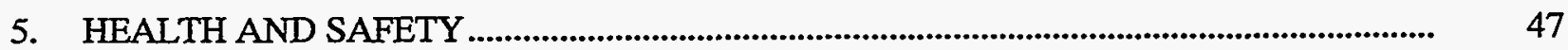

5.1 Safety and Health for Phase I Testing ..................................................................... 47

5.2 Environment, Safety, and Health for Phase II Testing .......................................... 48

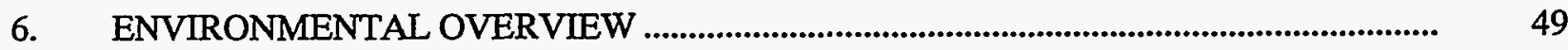

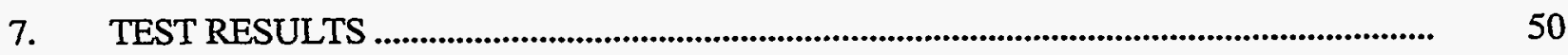

7.1 Phase I Demonstration Test Results ..................................................................... 50

7.1.1 Contamination Control Effectiveness ............................................................ 50

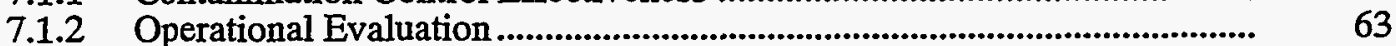

7.1.3 Effectiveness on Various Waste Forms ........................................................ 73

7.1.4 Overall Phase I Demonstration Success ....................................................... 75

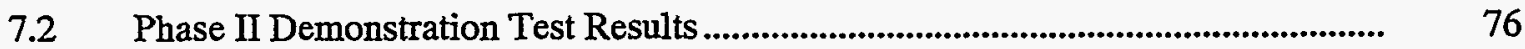

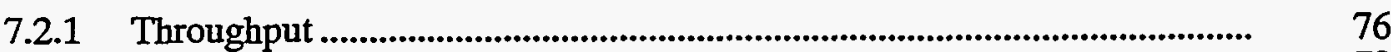

7.2.2 Effectiveness on Large Objects ................................................................. 79

7.2.3 Effectiveness on Various Waste Forms ......................................................... 83

7.2.4 Effectiveness of Telerobotic Control System................................................ 84

7.2.5 Maneuverability and Capability on Rugged Terrain, Precision and 97

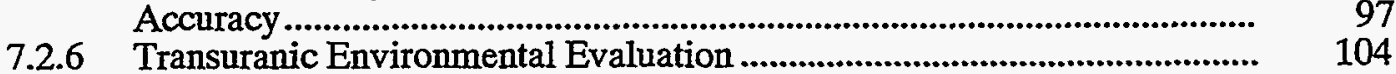

7.2.7 System Integration ...................................................................................... 105

7.2.8 Overall Phase II Demonstration Success....................................................... 111

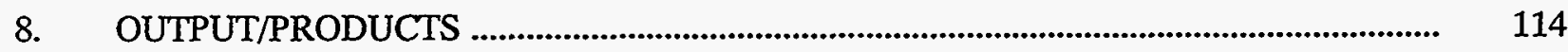

$8.1 \quad$ Theoretical Output of Process ........................................................................................ 114 
8.2 FY-94 Output........................................................................................................... 114

8.2.1 Data Output ...................................................................................... 114

8.2.2 Physical Output ........................................................................................ 114

8.2.3 Technology Transfer and CRADA Opportunities............................................. 114

9. PROBLEMS AND ISSUES FROM THE TESTING .............................................................. 115

9.1 Telerobotic Transport Vehicle ...................................................................................... 115

9.2 Innovative End Effector.......................................................................................... 117

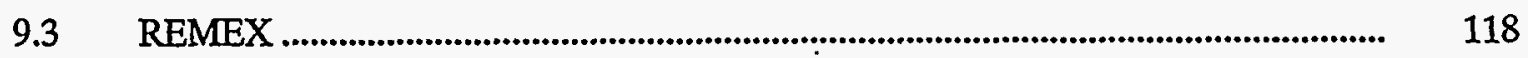

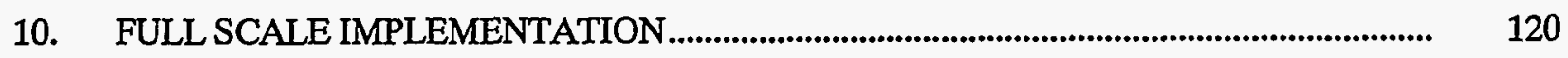

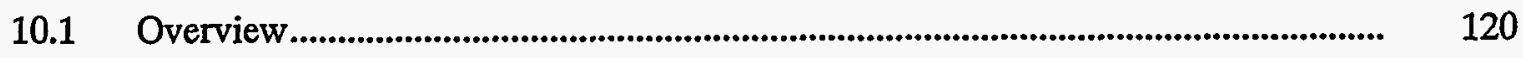

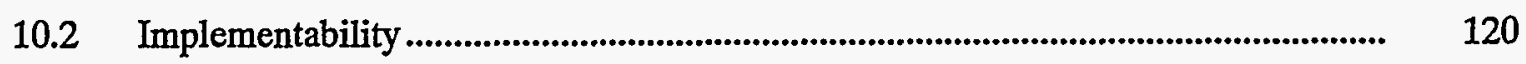

10.2.1 Ability to Construct and Operate the Technology .................................... 120

10.2.2 Reliability of the Technology ........................................................................ 121

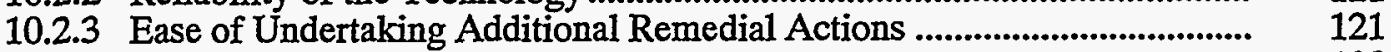

10.2.4 Ability to Monitor Effectiveness of Remedy ............................................. 122

10.2.5 Ability to Obtain Approvals from Other Agencies .................................... 122

10.2.6 Coordination with Other Agencies .................................................................. 122

10.2.7. Availability of Offsite Treatment, Storage, and Disposal Services and 122

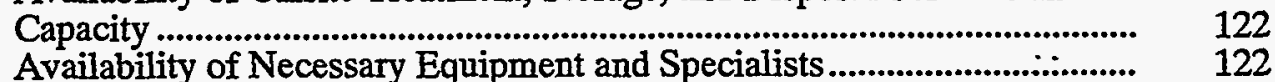

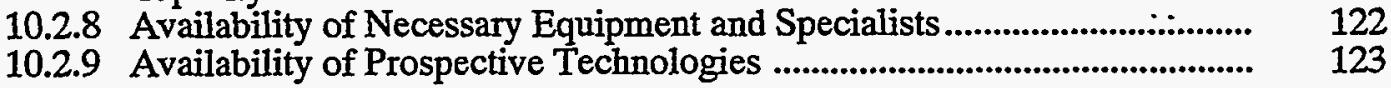

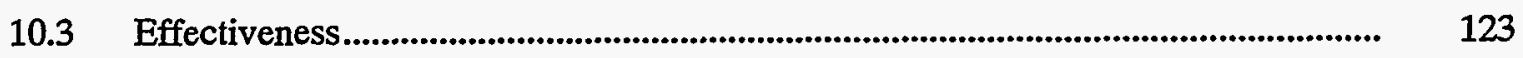

10.3.1 Overall Protection of Human Health and the Environment ........................ 123

10.3.2 Long-Term Effectiveness ............................................................................... 124

10.3.3 Reduction of Toxicity, Mobility, and Volume ........................................... ' 124

10.3.4 Short-Term Effectiveness ................................................................................... 125

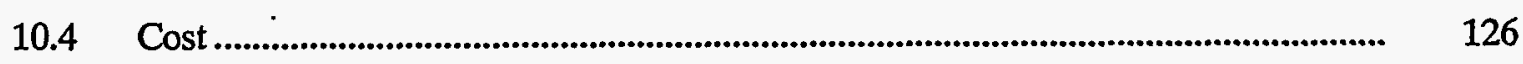

10.4.1 Capital Costs...................................................................................... 126

10.4.2 Operating and Maintenance Costs .................................................................. 127

11. CONCLUSIONS AND RECOMMENDATIONS ........................................................... 129

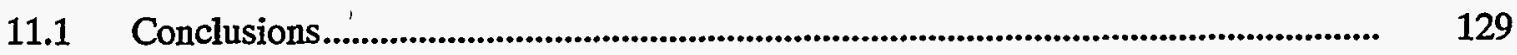

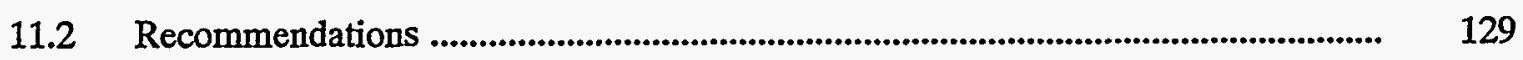

11.2.1 Innovative End Effector........................................................................ 129

11.2.2 Conveyance System .......................................................................... 129

11.2.3 REMEX .............................................................................................. 131

11.2.4 Overall Test Layout and Remote Control Station ............................................ 131

11.2.5 Theoretical/Overall................................................................................ 132

11.2.6 Design Modifications for a TRU Environment ................................................. 132

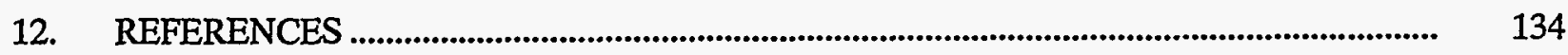


Appendix B—Final Acceptance Test Records

Appendix C-Derivation of the Dust Level

Appendix D-Chemistry Laboratory Report, Dust and Rare Earth Tracer Analysis

Appendix E-Corrected Test Procedures

Appendix F-Dust and Rare Earth Tracer Analysis.

Appendix G-Phase I Detailed Test Data

Appendix H-Phase II Detailed Test Data

\section{FIGURES}

1. Process diagram showing a portion of the waste retrieval process ......................................... 3

2. The IEE is highly integrated with the excavator and remote conveyance system

3. The multipurpose end effector integrates innovative features with proven excavation and retrieval technology

4. The tapered design of the ITM and waste transport container facilitate the transfer process.

5. The ITM is a detachable loading, handling, and conveyance bucket

6. The waste transport container carries the ITMs and can be used to transport large objects including intact $4 \times 4 \times 8$-ft waste boxes

7. The TTV mechanical subsystem consists of a track assembly, main frame, diesel power plant, hydraulic system, and ITM cradle.

8. The Hitachi EX-200LC REMEX was modified to a front shovel configuration for belowgrade excavation

9. The human factors designed control arm is the same in the excavator cab as it is in the remote control station

10. The Phase I test was performed inside a building where wind would not affect the dust and rare earth tracer measurements

11. The Phase I simulated waste pit included INEL soil, 55-gal drums, paper, concrete, and steel beams

12. The Phase II test was performed outdoors and the TTV test path included a 10 degree slope

13. The Phase I test was conducted in an enclosed structure to ensure controlled environmental conditions.

14. The Phase II test involved exhumation of simulated buried TRU waste.

15. The dust measurement graph for test 5 shows the test sample levels were considerably less than target of $1.1 \times 10^{-4} \mathrm{~g} / \mathrm{L}$-air above the detected background levels 
16. The rare earth tracer quantities graph for test 5 shows the praseodymium test sample levels and the background levels detected

17. The test means for the five dust measurement tests clearly show that all of the dust samples taken are significantly (two orders of magnitude) below the target goal of $1.1 \times 10^{-4} \mathrm{~g} / \mathrm{L}$-air

18. The average dust collected for each air monitor during the demonstration clearly shows that HV-9, the monitor closest to the digging, had the highest average dust loading

19. Placement of the air monitors for the Phase I demonstration was intended to isolate the dust generation caused by digging from that caused by dumping.

20. Test and background means for the five rare earth tests show that tracer was

detected confirming the movement of particles of praseodymium from the source.

21. The average tracer collected for each air monitor during the demonstration shows that tracer was detected at levels significantly above background

22. Dust and rare earth tracer data show an almost perfect correlation ( 0.98 with 1.0 being perfect) for the demonstration.

23. Before modifications, the IEE had a large overflow slot and teeth on the bucket and thumb

24. The IEE was more effective at handling the waste forms and filling the ITM after minor modifications were made.

25. The IEE was flexible in its ability to pick up drums in two ways: either by depositing the drums into the attached ITM (as shown) or by using the bucket and thumb to pick up the drum and then deposit it into a detached ITM

26. Attaching the ITM to the IEE is aided by the matching tapered designs

27. The pick-up truck bed was manipulated to size it to fit into the WTC using the IEE ..............

28. The remote control station employed existing equipment and considered human factors design as much as possible:

29. The test site terrain included a 10 degree slope and a variable terrain with soft sandy soil.

30. The TTV's ability to meet the precision and accuracy goal of 1-radial foot was affected by crosstalk between the ultrasonic sensors.

31. The capacity of the WTC allowed it to close over the ITM even when items in the ITM protruded above the top of the ITM

32. The spring bed on the TTV aided in attaching empty ITMs to the IEE because it provided flexibility in all three directions.

33. The TTV docked at the target with the aid of ultrasonic sensors 


\section{TABLES}

1. Potential DOE application areas for the remote excavator/IEE and conveyance systems

2. Data and analysis for the dust measurement for test 5

3. Data and analysis for the rare earth tracer quantities test 5

4. The statistical analysis strongly supports the null hypothesis that the mean dust detected was, for all tests, less than the test goal of $98 \%$ dust removal.

5. The statistical analysis supports the alternative hypothesis that the mean tracer detected was, for most tests, greater than the background levels

6. Summary of data for Phase I testing

7. Summary of production rates and equipment cycle times for Phase II testing

8. Estimated capital equipment costs 


\section{ACRONYMS}

ALARA As low as reasonably achievable

ARAR Applicable or relevant and appropriate requirements

BWID - Buried Waste Integration Demonstration

CERCLA Comprehensive Environmental Response, Compensation, and Liability Act

CPU Central Processing Unit

CX Categorical Exclusion

DOE U.S. Department of Energy

FCC Federal Communications Commission

FY Fiscal year

HI-VOL High-volume air sampler

ICP-MS Inductively Coupled Plasma Mass Spectrometry

IEE Innovative End Effector

INEL Idaho National Engineering Laboratory

ITM Integrated Transfer Module

LITCO Lockheed Idaho Technologies Company

LCD Liquid crystal display

MSDS Material Safety Data Sheets

NEPA National Environmental Policy Act

OTD Office of Technology Development

PPE Personal Protective Equipment

QA Quality assurance

QC Quality control

REMEX Remote Excavator (SPAR's Hitachi EX200LC)

RF Radio frequency

ROD Record of Decision

RWMC Radioactive Waste Management Complex

SDA Subsurface Disposal Area

TRU Transuranic 
TTV Telerobotic Transport Vehicle

VARSA Vehicle Acoustic Ranging System

WTC Waste Transport Container 


\section{An Integrated Systems Approach to Remote Retrieval of Buried Transuranic Waste Using a Telerobotic Transport Vehicle, Innovative End Effector, and Remote Excavator}

\section{TECHNOLOGY DESCRIPTION}

Due to a contractor change at the Idaho National Engineering Laboratory (INEL), this project was initiated under the administration of EG\&G Idaho, Inc., and completed under the administration of Lockheed Idaho Technologies Company (LITCO). The contractor change took place on October 1, 1994. The demonstration was completed on September 30, 1994, with the data analysis and final report completed in January 1995.

\subsection{Background}

Between 1952 and 1970, over two million cubic feet of transuranic (TRU) mixed waste was buried in shallow pits and trenches in the Subsurface Disposal Area (SDA) at the Idaho National Engineering Laboratory (INEL) Radioactive Waste Management Complex (RWMC). Commingled with this two million cubic feet of waste is up to 10 million cubic feet of fill soil. ${ }^{1}$ Approximately $6 \mathrm{ft}$ of clean overburden soil has been placed on top of the buried waste pits and trenches to isolate the waste from surface water runoff and wind erosion. The pits and trenches were constructed similarly to municipal landfills with both stacked and random dump waste forms such as barrels and boxes. The barrels are typically 55-gal drums made of metal. The boxes are typically $4 \times 4 \times 8 \mathrm{ft}$ and constructed of plywood. The drums and boxes contain wastes such as paper, sludge, metal, tools, cloth, wood, asphalt, concrete, and glass. There could also be large objects including storage tanks, vehicles, pipes, beams, and glove boxes. The main contaminants are micron-sized particles of plutonium and americium oxides, chlorides, and hydroxides. ${ }^{2}$ Retrieval, treatment, and disposal is one of the options being considered for the waste. As an aggregate, the retrieved waste will average above $10 \mathrm{nCi} / \mathrm{g}$ for the TRU elements. The waste also contains volatile organics such as trichloroethylene and carbon tetrachloride and cutting oils such as REGAL oil. The waste containers are assumed to be severely deteriorated to the point that many containers are breached, leaking, or even unrecognizable because of deterioration.

The configuration of waste described above and located at the SDA is similar to other buried waste located at U.S. Department of Energy (DOE) sites throughout the United States. In response to the need to remediate these sites, the DOE's Office of Technology Development (OTD) has initiated a comprehensive program to develop and demonstrate advanced remediation technologies that support DOE's environmental restoration objectives. Further, OTD has initiated the Buried Waste Integrated Demonstration (BWID) Program at the INEL to investigate the feasibility of new and existing technologies for the effective in situ treatment, removal, and treatment after removal of buried wastes. BWID will address the complex problems associated with buried waste by initially focusing on buried waste located at the INEL. Technologies that are successfully developed within the BWID Program will result in the transfer of scientifically sound, demonstrated technologies that can be deployed in various Environmental Restoration programs throughout the DOE complex. 


\subsection{Purpose}

The overall objective of the demonstration was to explore the effectiveness of methods and equipment to remotely retrieve and transport buried waste away from the digface and control dust generation and contamination spread while dumping retrieved waste. The data will eventually be used by Environmental Restoration during the feasibility study for parts of the INEL's RWMC and, possibly, by additional remediation efforts at other DOE locations and U.S. Superfund Sites. Data will also be used by Environmental Restoration and BWID to project additional technology needs for BWID out-year planning.

The waste conveyance system and innovative end effector (IEE) are major components of the retrieval related technologies. These technologies directly affect the digging, dumping, and transport processes of the retrieval (see Figure 1).

Previous experiments conducted at the INEL have used both misting systems and directed airflow to control the spread of dust during dumping operations into a funnel. Misting and directed airflow, while practical, require more maintenance and downtime for repair and are cumbersome to deploy, maintain, and move. It is desirable to avoid directed airflow and/or misting systems if possible. Neither of these approaches are ideal because in both cases dust escapes regardless of airflow or misting rate. In addition, the dust that does escape is the respirable fraction thought to carry the plutonium/americium particles. The dumping of dry soil into a funnel/dumpster arrangement has been found to be the primary mechanism for dust generation during the retrieval process. Therefore, improving the method of dumping soil and debris and thereby limiting the spread of dust and contaminants are mandatory.

Regardless of the excavation technique, exhumed waste will have to be conveyed away from the retrieval area to the packaging area or directly to the treatment facility. Conveyance of the retrieved waste from the digface during the fiscal year (FY)-1993 conventional retrieval demonstration was performed by an integrated tool carrier (Caterpillar IT-28) with a forklift attachment. Although the vehicle used in the demonstration was effective in traversing the terrain and lifting the loads, it is not remotely controlled as will be required in a radioactive (hot) retrieval.

To address this need, an IEE and telerobotic transport vehicle were designed and tested during FY-94 by RAHCO International and SPAR Aerospace Ltd. for the BWID program funded by OTD.

The IEE system and remotely operated conveyance system were developed in close coordination with one another to ensure a systems design approach that maximizes the effectiveness of the combined technologies. The following describes the relationship between the demonstrations and the envisioned actual operation:

- The demonstration was limited in the types of waste materials that were included in the excavation process whereas the actual operation would have a full range of waste items and materials that would be encountered.

- The demonstration was performed in a cold environment as opposed to a hot or radioactive environment found in an actual operation. This prevented unnecessary delays or downtime for extensive decontamination during maintenance and possible retooling. 


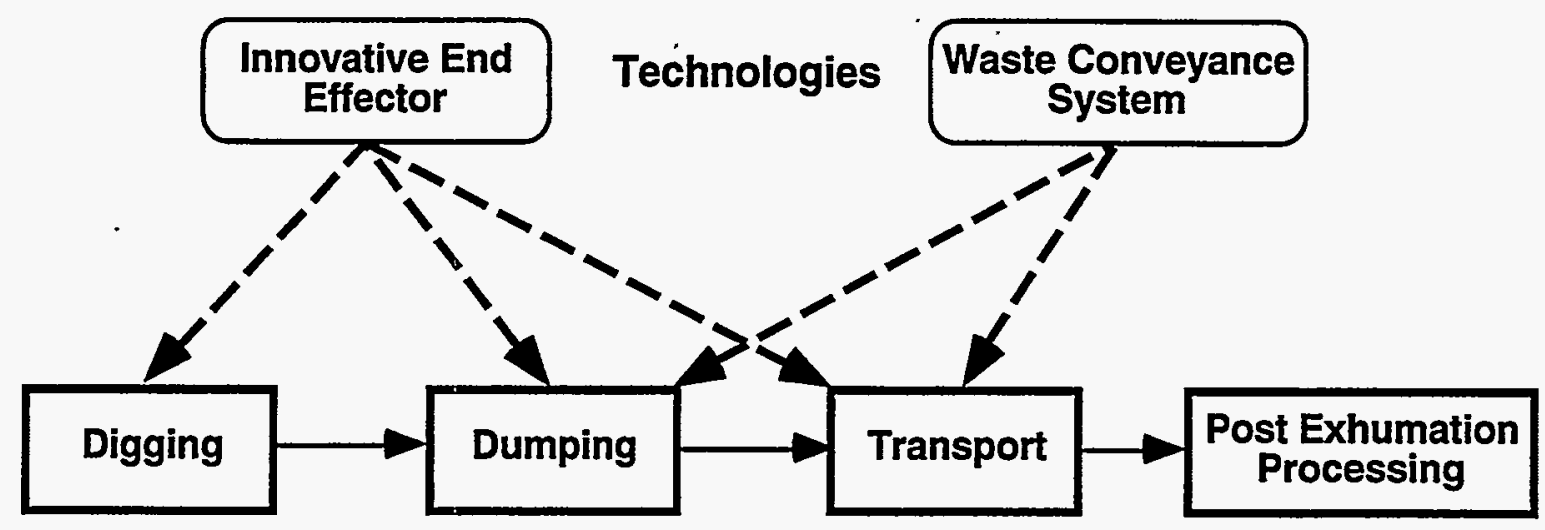

Waste Retrieval Process

Figure 1. Process diagram showing a portion of the waste retrieval process. Waste conveyance and IEE . technologies affect several stages of the retrieval process.

\subsection{Apparatus}

\subsubsection{Innovative End Effector}

A specially designed end effector that features a thumb and an attachable/detachable Integrated Transfer Module (ITM) and a rugged, universal transport container capable of handling up to 9,000 lb of waste were developed to transfer the waste away from the digface (see Figure 2).

The end effector consists of two structural assemblies: the inverted thumb and the shovel assembly (see Figure 3). The necessary actuating linkage and hydraulic cylinders are also part of the end effector. The upper structural assembly consists of a hydraulically actuated thumb. This type of device has been proven to be extremely versatile, dexterous, and productive in handling the expected waste forms. ${ }^{3}$ The front shovel configuration with the thumb on top was chosen to increase digging efficiency, reduce dust generation, and minimize clean up when the shovel digs into the face rather than pulling away from the digface. The lower shovel assembly, consisting of digging teeth, a guide skirt, an overflow opening, and a latching mechanism, has multiple functions. When used with the thumb, it can retrieve large items and guide 55-gal drums into the ITM. It also acts as a shovel to scoop dirt and debris into the ITM. To prevent the ITM from overfilling, the shovel assembly has a small overflow opening between the teeth and top of the ITM. The shovel assembly also has a flared skirt with a 3-in. draft to accommodate easy attachment of an empty ITM to the shovel assembly (see Figure 4). A latch mechanism on each side of the shovel assembly secures the ITM to the assembly. Each latch is equipped with hydraulically actuated latches that lock the ITM to the shovel assembly. This provides a positive, fail-safe connection.

The attachable/detachable ITM acts as a detachable loading, handling, and conveyance bucket when digging, handling, and conveying 55-gal drums, dirt, and small debris (see Figure 5). It is a rectangular, welded steel structure and holds approximately $1.4 \mathrm{yd}^{3}$ of soil and waste, or two 55-gal drums. A flared skirt around the upper portion is similar to that on the shovel assembly and assists the operator in mating the ITM with the shovel assembly. The tapered bottom assists the operator in placing the ITM into the transport container pocket. Slots in the ITM top flanges allow the shovel assembly dogs to be engaged and lock the unit to the shovel assembly. 


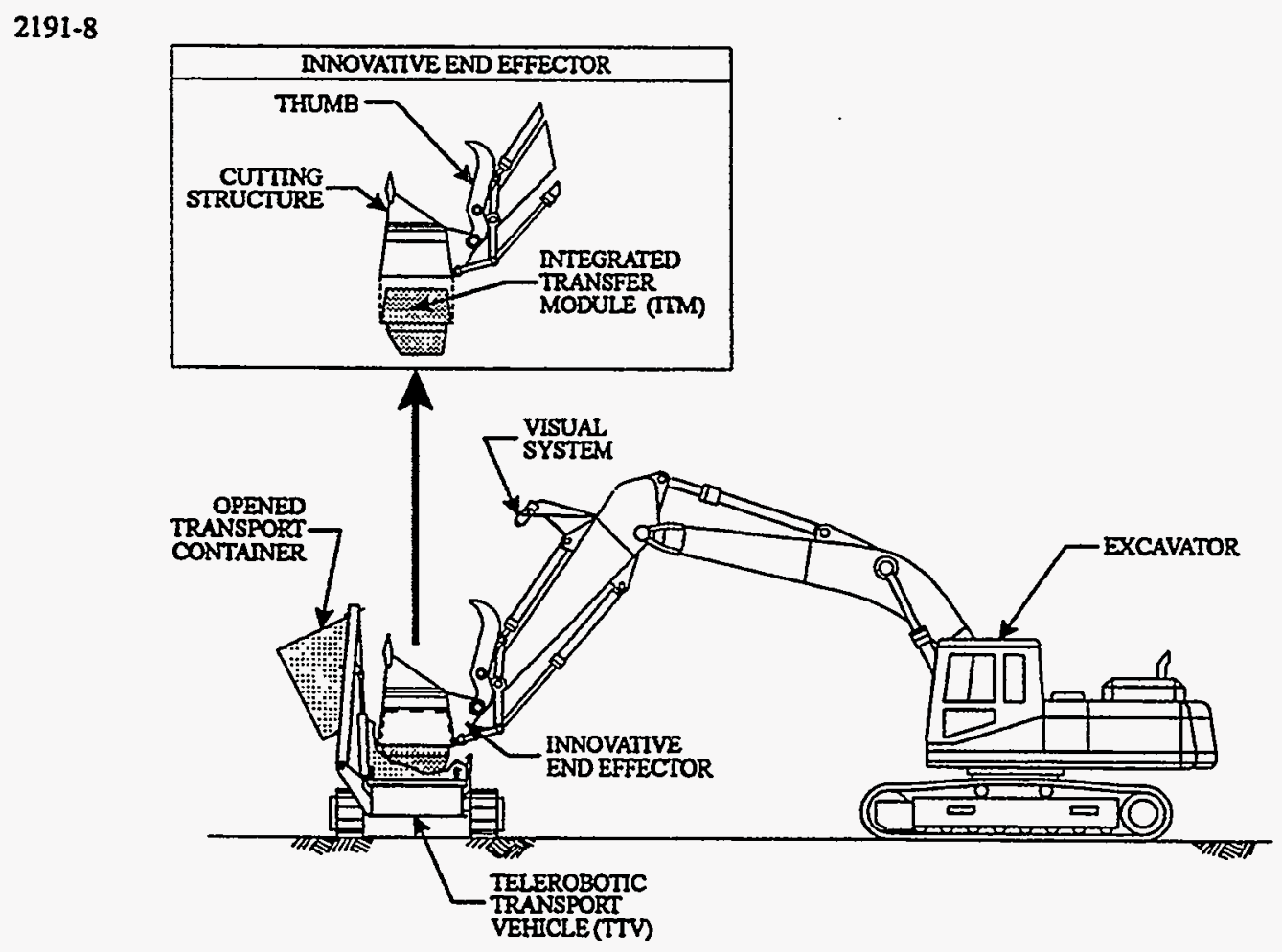

Figure 2. The IEE is highly integrated with the excavator and remote conveyance system.

$2191-4$
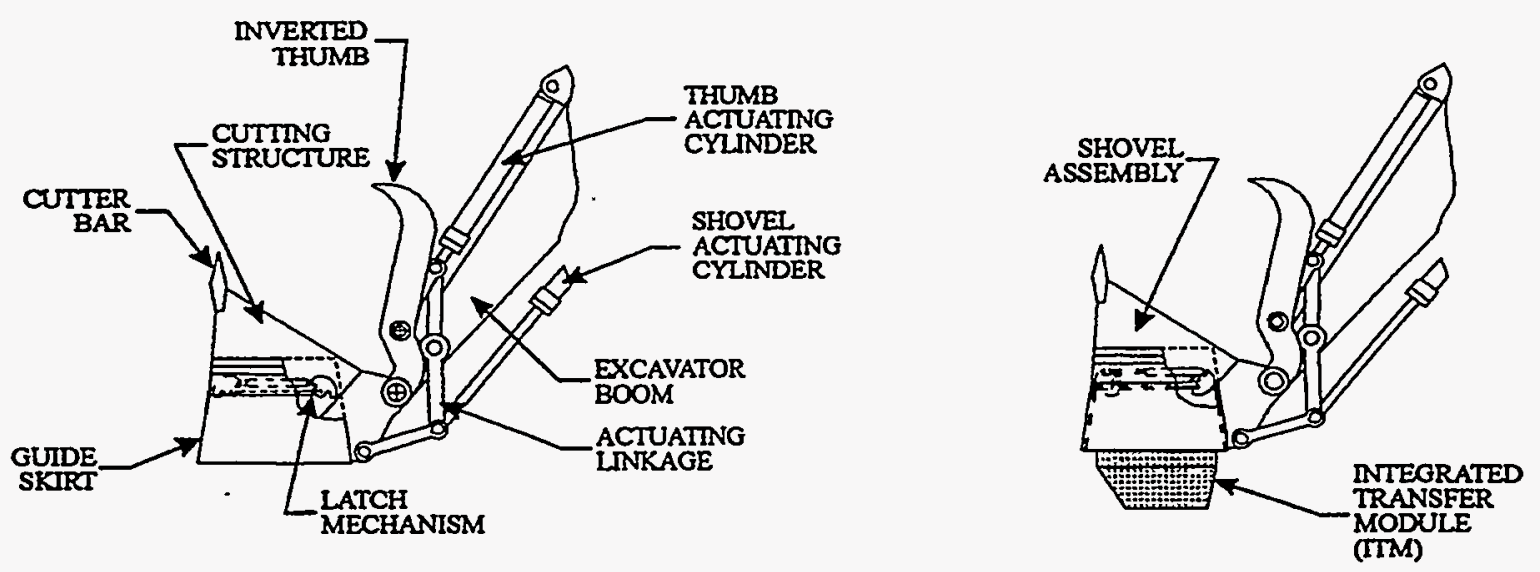

Figure 3. The multipurpose end effector integrates innovative features with proven excavation and retrieval technology. 


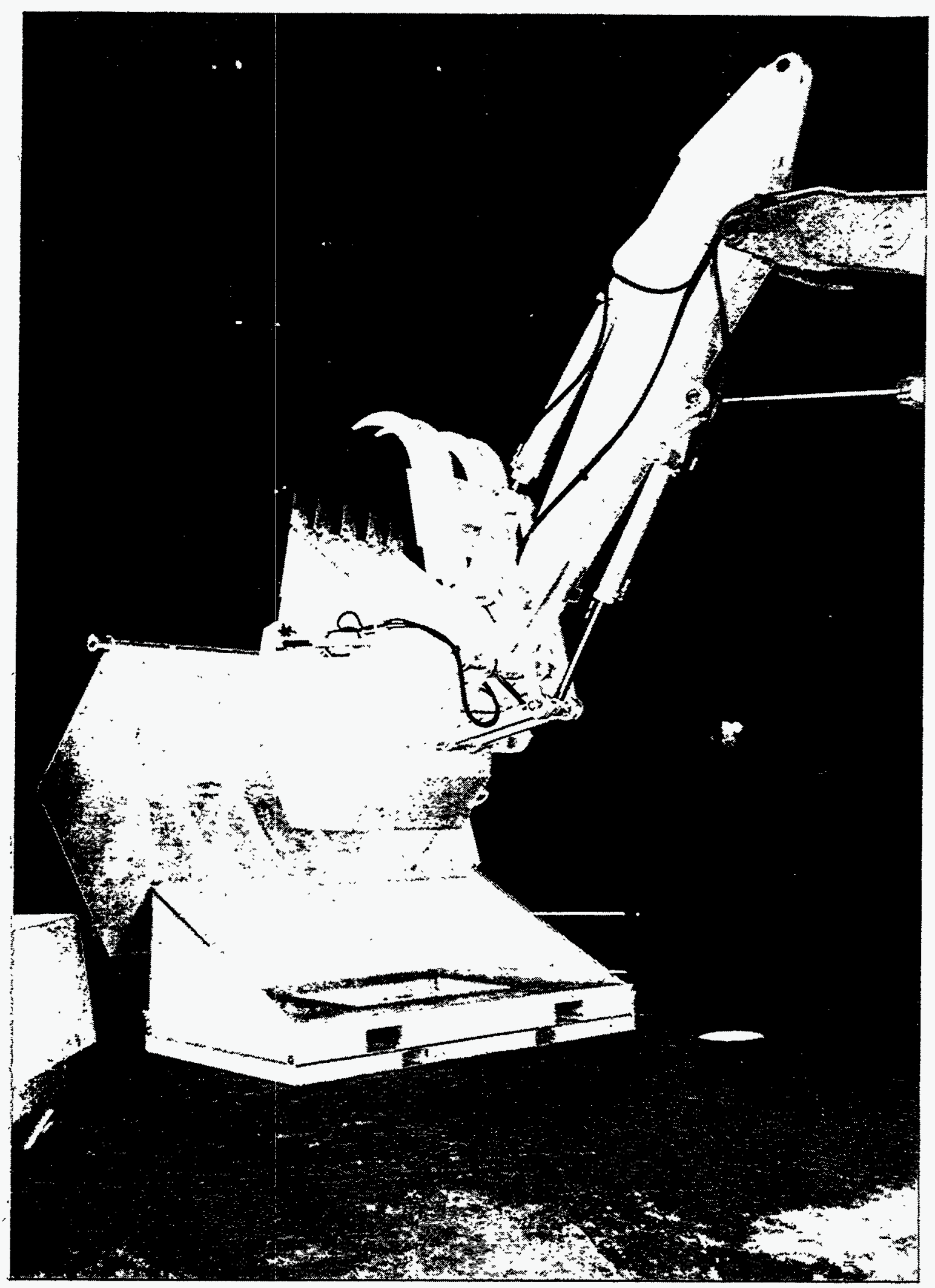

Figure 4. The tapered design of the ITM and waste transport container facilitate the transfer process (INEL 94-1187-7-11). 


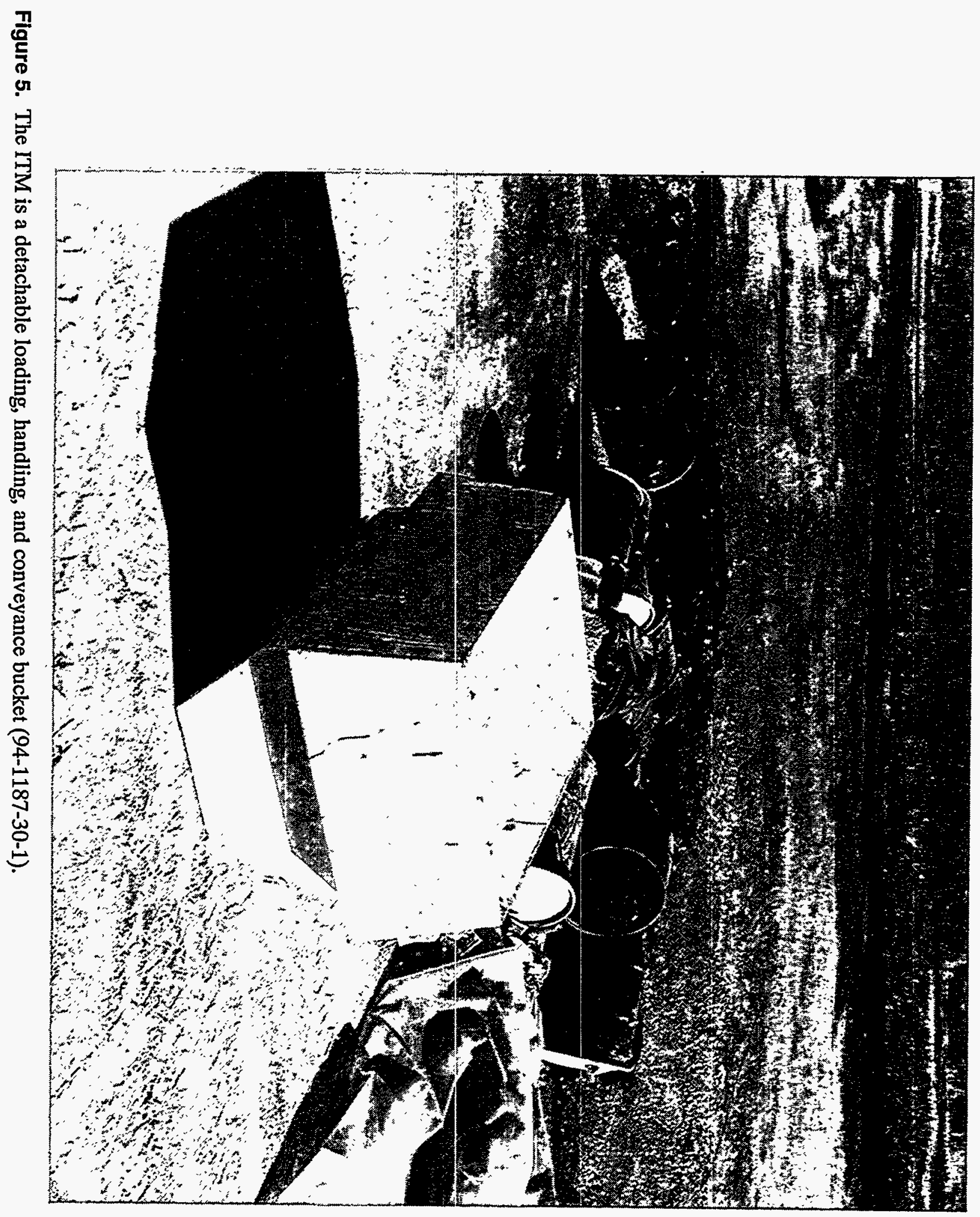


The 9,000-1b capacity transport container receives retrieved waste in the form of filled ITMs, $4 \times 4 \times$ 8-ft boxes, and many other expected, irregularly shaped, large waste items (see Figure 6). After receiving the waste items directly or in an ITM, the container is closed and automatically sealed and locked to contain any dust generated during conveyance. The container is a steel structure approximately $5 \mathrm{ft}$ wide, $5 \mathrm{ft}$ high, and $9 \mathrm{ft}$ long, weighing approximately 2,800 lbs empty. The rectangular lid structure is connected to the base structure by a simple hinge assembly. The lid structure is opened by a lift mechanism to provide frontal access, thus allowing easy placement of the ITM or other waste items by the excavator operator. A tapered pocket, located in the bottom of the container, is configured and sized to provide an easy target for the operator and self-aligns the pocket and ITM during loading. Dust seals, located on all sealing surfaces, are provided to contain the dust generated during conveyance. A springloaded latching mechanism engages the lift bar on the lid to lock and secure the lid during handling, preventing inadvertent opening of the container. In operation, the end effector selectively retrieves the expected waste forms and places the collected waste in a 9,000-lb capacity container. For large waste items, the end effector places the item directly onto the container floor. For dirt, debris, and 55-gal drums, the excavator scoops the materials into the ITM, swings the filled ITM over the transport container, and lowers the ITM into the container pocket where it is detached. An empty ITM, staged on the Telerobotic Transport Vehicle (TTV), is then attached to the end effector and swung back to the digface. Simultaneously, the container lid is closed, and the filled ITM is secured in the container. The ITM is then carried by the TTV to a storage area receiving station where the filled container and enclosed ITM are off loaded. An empty ITM, along with an empty container, are loaded onto the TTV. The TTV then travels back to the digface.

\subsubsection{Remote Conveyance System}

The remote conveyance system was designed and provided by RAHCO. It consists of a trackmounted TTV and a remote operator control station. The TTV transports the loaded/unloaded transport containers and ITMs. The remote operator control station is used for controlling the TTV, remote excavator, and video/audio systems. The TTV is fully integrated with the end effector/waste handling system to provide a waste conveyance solution that eliminates dust generation during conveyance operations. The TTV, as shown in Figure 7, is designed as a multiple function vehicle. First, it conveys full containers and ITMs from the excavation location to the dumping/unloading location. Second, it serves as a transfer station at the excavation site by (a) supporting the transport container, (b) opening, closing, and locking the container lid, and (c) providing a staging platform for the empty ITM. Third, it conveys the empty containers and ITMs from the storage area to the excavation site. The TTV consists of the mechanical, guidance, and control subsystems.

A main frame connects the tracks and supports the loads. One track is mounted rigidly to the frame while the second track is connected by horizontal pivot pin. This minimizes racking of the frame as the TTV travels over irregular terrain.

A nonpowered roller table is located on top of the main frame. On an operational transporter, the rollers will be powered and used for rapid off loading of filled containers and on loading of empty ITMs and containers.

Located at one end of the TTV is an ITM cradle. The cradle is a three-sided steel frame supported on four large springs. This spring-mounted structure allows the ITM to self-align with the end effector during the ITM-end effector attachment operation. 


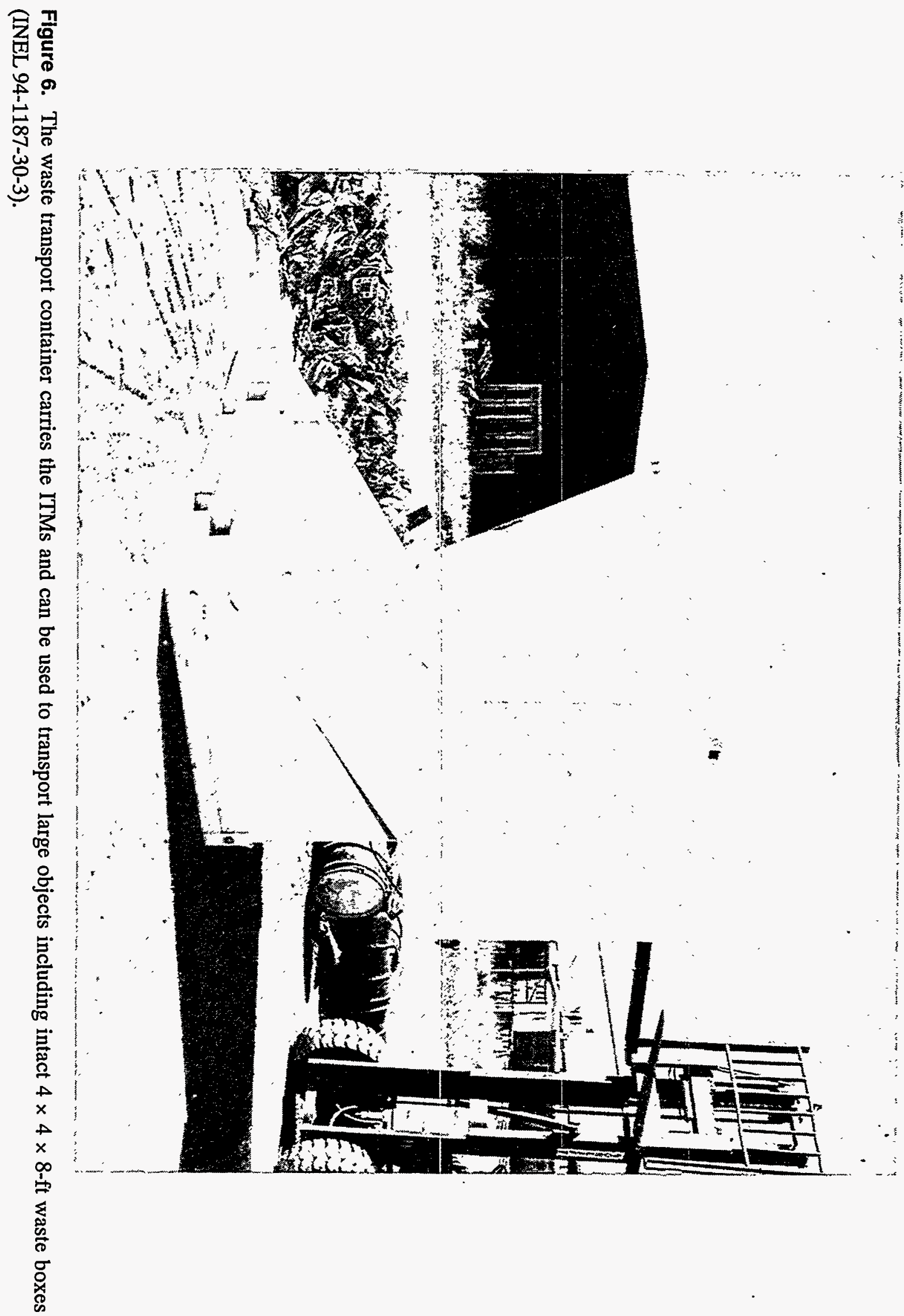




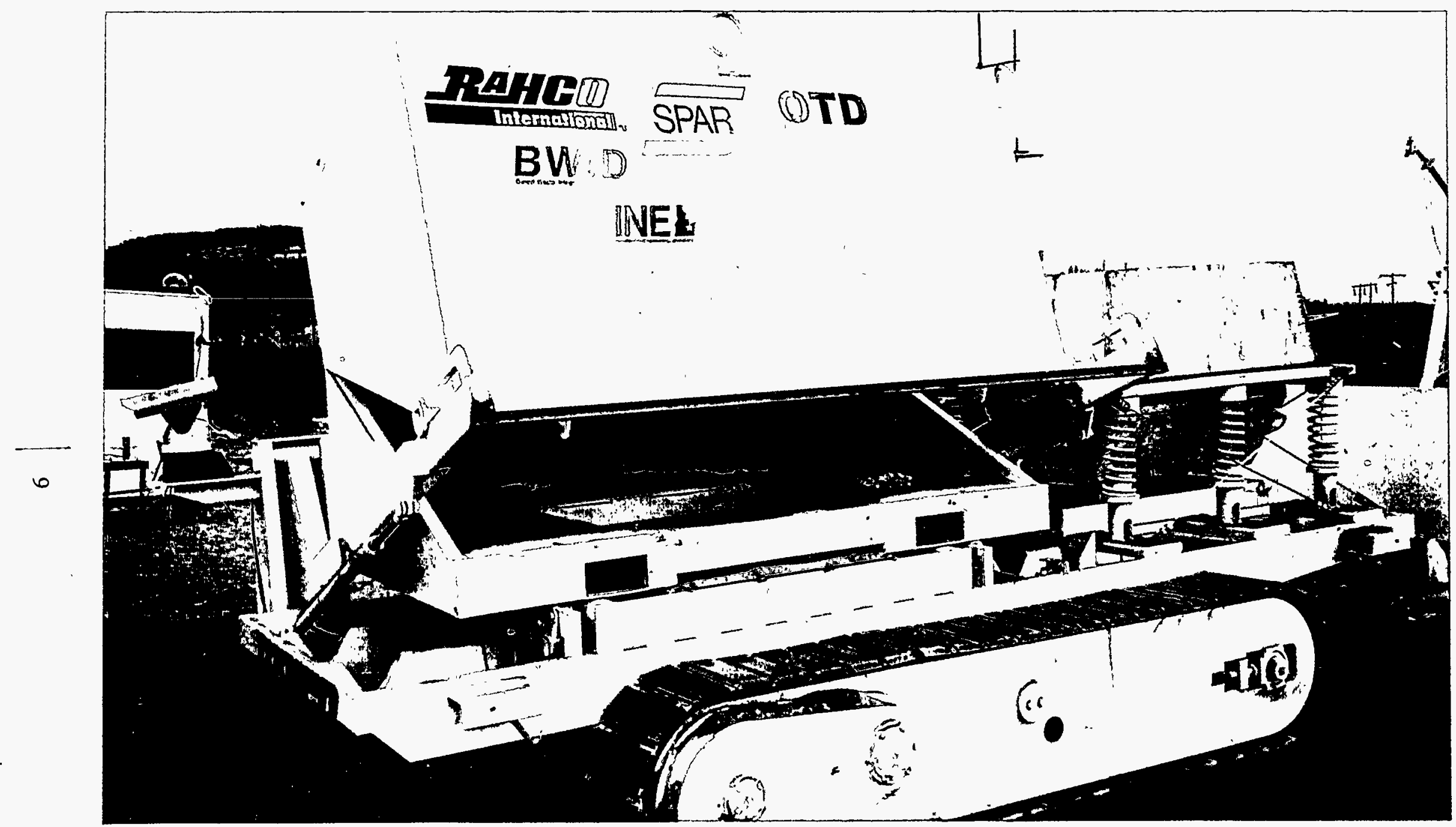

Figure 7. The TTV mechanical subsystem consists of a track assembly, main frame, diesel power plant, hydraulic system, and ITM cradle. The TTV can travel at speeds to $240 \mathrm{fpm}$ on a set of hydraulic motor-powered crawler tracks. The tracks provide stability on slopes and excellent maneuverability. Employing skid steering, the TTV is designed to turn 360 degrees about its own center on a 14-ft wide roadway. The mechanical subsystem uses existing, state-of-the-art, mobile equipment components (INEL 94-1187-8-19). 

A lift table, in the raised position, protrudes between the rollers and holds the transport container above the rollers. This protects the rollers from impact during the loading of waste into the containers.

Two hydraulically actuated lift mechanisms mounted to the TTV main frame release the transport container lid latches and raise the lid structure.

The TTV is powered by a 47-hp, water-cooled, diesel engine. A belt-driven, 24-V dc generator provides electrical power for the control system. The engine is directly coupled to a variable displacement hydraulic pump that powers the TTVs various hydraulic components.

The SPAR-provided guidance and control systems consist of

- TTV Control System

- Operator Control Station

- Three radio frequency (RF) links between the TTV control system and the Operator Control Station

- $\quad$ Remote Operations Control Trailer.

The TTV control system has three separate microprocessor controllers to provide dedicated track control, waste container operation/control, and selected control of the forward and rear-mounted vehicle cameras with pan and tilt units. Each microcontroller has a resident INTEL 80C196K microprocessor running proprietary software and shares a common RF link to the operator control station. These are rugged, field-proven units developed from grapple-yarders used in the logging industry.

The transporter also has on-board sensing systems to provide track speed transduction, a rate gyro for heading and noise filtering around the speed transducers, waste handling logic function switches, and a Vehicle Acoustic Ranging System Array (VARSA).

A video system, comprised of forward and rear-mounted cameras with pan and tilt units, gives the remote operator visibility in forward and reverse directions. The video system is used for steering control and tracking of specially designed acousto-video targets. The targets provide the TTV operator vision tracking to allow execution of the 90 degree turn required to align the waste transport container (WTC) with the excavator and receiving station.

Three vehicle control modes are provided:

\section{Mode 1: Open Loop Track Control}

Tracks are operated from the vehicle-mounted manual control or from the remote operator's joystick if the normal telerobotic control function either fails or is not required.

\section{Mode 2: Telerobotic Control}

Telerobotic control provides resolved control of tracks through a single joystick at the remote operator's station. The joystick commands provide proportional forward/reverse control and left/right directional control. The microprocessor at the operator's station resolves joystick 
commands into track speed commands that are sent by the RF link to the vehicle track microcontroller. The track microcontroller integrates the track speed commands with speed transducer and rate gyro feedback to control the tracks.

\section{Mode 3: Waste Container Operation}

This mode provides loading and unloading while the TTV is stationary. Operator commands from the remote control panel initiate individual control functions such as lid unlatch and open/close and latch. The on-board control system also monitors limit switches to ensure each function has also been completed.

For teleoperated control, the transporter is operated from the remote operator control station. The control station consists of a microprocessor, control panel, RF modem, video monitor/controller, and graphical overlay controller.

The control panel includes

- One joystick providing input commands for the vehicle direction and speed in either mode 1 or 2

- Mode Select Switch allowing Off/Mode 1/Mode 2/Mode 3 selections

- Camera select and pan and tilt switches

- Switches for lid open/close and latching

- Emergency stop button with key lock

- Keypad for setting parameters including acoustic array ranging limits

- Liquid crystal display showing system health monitoring messages and status

- Spread spectrum radio modems providing RF telemetry for operator commands, emergency stop, and video signals.

Operation of the TTV can be carried out using Modes 1 and 2 at the remote operator's station or independently at the TTV itself using a pendant mounted controller. An emergency stop button is also provided on the TTV. For the waste retrieval demonstration, the operator controlled the vehicle in the telerobotic Mode 2 using the single joystick for travel control and the forward or rearward cameras for visibility.

As the TTV approached the excavator, the operator tracked to an acousto-video target set on free standing targets. The ultrasonic sensors were able to transduce range when they were within .6 to $35 \mathrm{ft}$ of the target.

A graphical overlay was displayed on the operator control station monitor. The overlay provided the operator with an image to track the target in to. Boundary conditions on the overlay provide the operator 
with alignment and approximate ranging data, confirming the data provided by VARSA and displayed on the panel's liquid crystal display (LCD). From this, the operator determined the distance and attitude of the vehicle with respect to the targets.

Having reached a designated point with respect to the excavator, the operator commanded the TTV to pivot 90 degrees and align to a free-standing set of targets. This positioned the TTV so the ITM could be placed in the transport container. The TTV was then moved to place the empty ITM at the correct location for attachment to the excavator end effector. The TTV returned to the unloading station where the vehicle was docked to a single, free-standing, acousto-video target.

Additional details about the TTV operation can be found in the TTV Operator's Manual in Appendix A and in Section 7. The detailed Final Acceptance Test Records for the performance tests can be found in Appendix B.

The Remote Operations Control Trailer consisted of a 20-ft, air-conditioned construction trailer. A generator provided electrical power.

\subsubsection{Remote Operated Excavator}

The Hitachi EX-200LC remote-operated excavator (REMEX) is a 40,000-lb class standard excavator that has been modified for remote operations (see Figure 8). Remote operations are accomplished by manipulating the umbilically tethered excavator from a remote operator's work station. A control arm allows for intuitive operations and eliminates the need for the operator to coordinate motions in several directions as required by standard excavators. In-cab operations have also been modified to use the human factors designed control arm (see Figure 9). Cameras mounted on the stick and cab provide video signals for remote operation. The REMEX was also modified for attaching the IEE instead of using a standard backhoe bucket. The system can be operated from a remote location up to $200 \mathrm{ft}$ away from the excavator by tether.

1.3.3.1 Excavator Features. The basic features of the Hitachi 2000 series excavator include

- $\quad$ All Up Weight-40,800 lb (min)

- Overall dimensions

- Stowed length-31 ft, 5 in.

- Cab height-9 ft, 4 in.

- Maximum radius - $30 \mathrm{ft}, 5 \mathrm{in}$. (at ground)

- Maximum elevation-30 ft

- Maximum depth-19 ft, 7 in.

- Vehicle width-9 ft, 10 in.

- Maximum forward speed-3.4 mph

- Automotive power-135 hp Isuzu turbo-diesel engine

- Transmission-2 speed axial piston motor/reducers. 


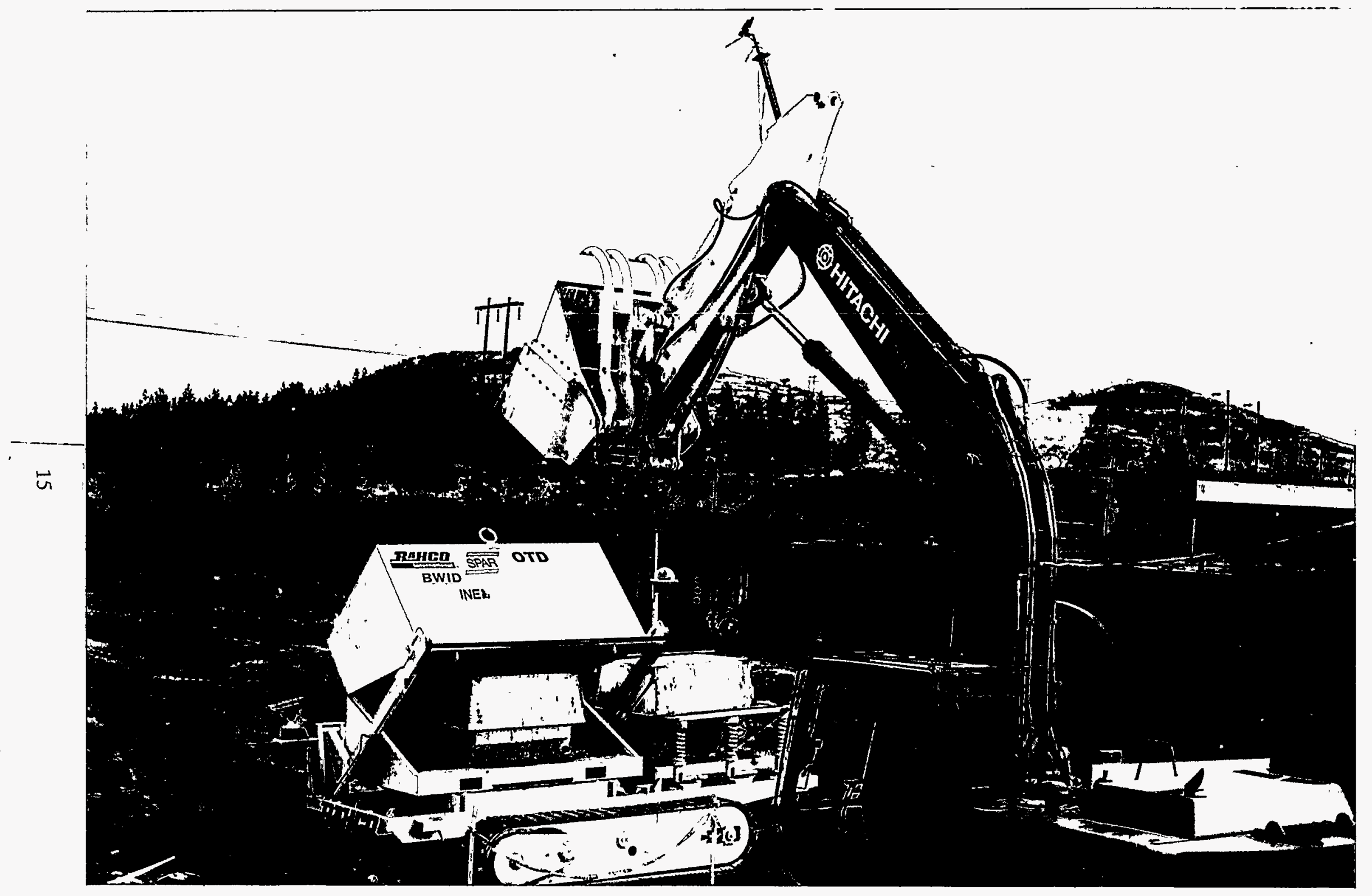

Figure 8. The Hitachi EX-200LC REMEX was modified to a front shovel configuration for belowgrade excavation (INEL 94-1187-28-4). 


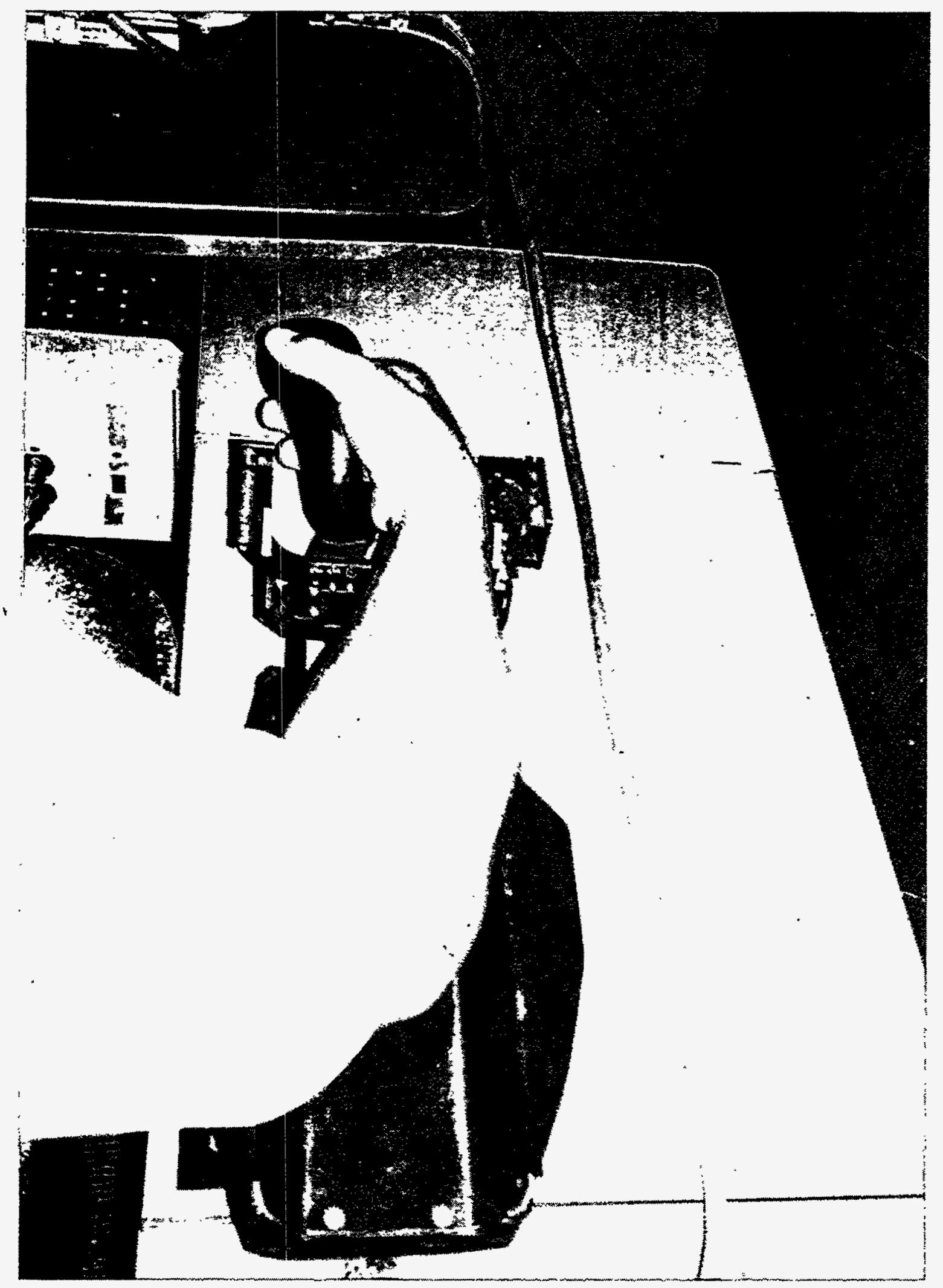

Figure 9. The human factors designed control arm is the same in the excavator cab as it is in the remote control station (INEL 94-1187-12-3). 
The standard excavator.was converted to a remote excavator by replacing the joystick commands for control valves by commands from a four degree of freedom hand controller at a remote operator's workstation. This was accomplished after performing inverse kinematics in a vehicle-mounted VME-based central processing unit (CPU). When operated in coordinated control mode, the CPU performs real-time kinematic transformation from inputs by ruggedized sensors added to the relevant joints and calculates the appropriate joint velocity derivatives to provide coordinated motion. Additional details on the control modes can be found in Section 1.3.3.2.

The remote excavator was further modified to accommodate the IEE, which reversed the bucket operation from backhoe to front shovel and added a thumb and latching mechanism for the ITM. Additional custom hydraulic circuits were designed and implemented together with simple remote controls. A special load limiting circuit was designed for the thumb so that the bucket control remained effective when clasping an object.

1.3.3.2 Excavator Operation. At the operator's remote workstation, a specially developed four degree-of-freedom hand controller was adapted from a unit developed for grapple yarders in the forestry industry. The hand controller was a prototype for this application but has evolved from a practical product. In addition to the hand controller, a dedicated workstation provides power and a command link to and from the excavator through the tether cable. At the workstation, a system-enable-switch is provided as well as a key lock emergency stop button and left and right track control levers. Within the console, another VME CPU manages hand controller coordinate commands in real time to provide proportional command outputs, data, and communications input/output between the workstation and vehicle CPU. The stock track control foot pedals are maintained.

The remote excavator can be operated in three different modes: manual, joint, or coordinated control. Joint control was used during this demonstration. All of the control modes use proportional control. Manual control is an open loop control system where the control signal received by the excavator is proportional to deflection of the joystick. In joint control, the excavator signals are servo controlled, so that the excavator moves with joint velocities that are proportional to the deflation of the joystick. When operating in joint control mode, the excavator control arm has four degrees of freedom. It can move left and right to swing the entire excavator. It moves up and down to control the boom. It moves in and out to control the stick. And lastly, the hand grip controls the pitch. In coordinated control, the control arm acts as an extension of the human arm, allowing for intuitive control. The computer calculates the coordination of the three excavator joints so that all three joints are moved to get a smooth, continuous motion relative to the joystick motion. In other words, with joint control, the operator must move the control arm in three different directions to keep the bucket level. In coordinated control, the operators move their hands in the direction they want the bucket to move, and the computer ensures that all of the joints move as required to implement this motion on the excavator. Coordinated control was not used for the demonstration because time did not allow the excavator to be tuned for the large-sized IEE.

There were four video systems. Two fixed cameras were mounted on tripods and provided orthogonal views of the digface area. There were two fixed cameras on the excavator, one on the stick and one in the cab. The cameras could not be remotely focused or zoomed in and out, and they did not have pan and tilt capability. 


\subsubsection{Test Venue and Layout}

Phase I of the demonstration was performed inside a building to simulate a retrieval environment. Dust and rare earth tracer spread were monitored using high volume (HI-VOL) air monitors. The test bed of simulated waste and INEL soil was constructed using concrete "Enviro blocks" to contain the waste. (Enviro blocks are stackable, mating blocks of concrete approximately $2.5 \times 2.5 \times 5 \mathrm{ft}$.) (See Figures 10 and 11.) The waste was excavated using the REMEX and IEE. The excavator tracks were moved as little as possible to reduce dust generation. The IEE attached to an ITM, dug a bucket of waste, and deposited the ITM for removal by a forklift. A second ITM was staged for the next bucket immediately after deposit of the first ITM. The full ITMs were removed for processing and recycling. HI-VOL air monitors were placed to monitor the spread of dust and rare earth tracer at the digging and dumping locations. A plywood wind break was constructed to reduce the amount of outside wind disturbance when the door was opened for forklift access.

Phase II of the demonstration was performed outside at a simulated waste pit. The excavation was done from belowgrade using the REMEX and the IEE. The TTV received a full ITM and delivered an empty ITM to the belowgrade location. The TTV then traveled to an abovegrade location to deliver the full ITM for processing and recycling and to receive an empty ITM. The remote excavator was run from a remote control station for the first $85 \%$ of the demonstration and from the operator's cab for the last $15 \%$ of the demonstration. See Figure 12 for a detailed layout of the Phase II test location.

\subsection{Technology Need}

The remote conveyance vehicle, REMEX, and IEE address two needs for buried waste retrieval. First, they are designed for remote operation, so that the operators are removed from the retrieval area, reducing the risk of exposure to hazardous and/or radioactive materials and fire and explosion. Second, they are designed to minimize dust during retrieval, primarily during the dumping stage. - Reducing dust reduces the level of airborne contaminants, which reduces the risk to workers and the environment. In addition, lower dust levels allow personnel wearing bubble suits to enter for maintenance activities. The REMEX deployed IEE and remote conveyance vehicle are designed to work together in an integrated fashion so that waste handling, spillage, and dust are all minimized.

\subsection{Customer Needs}

The DOE has established Environmental Restoration Programs that, in part, address the remediation of buried TRU waste located at sites around the United States. The purpose of this project was to develop, with an industrial partner, a control system for installation on a vehicle or other conveyance apparatus capable of negotiating the terrain expected in a waste retrieval area, for the purpose of remotely transporting retrieved waste from the point of excavation to post exhumation processing and an IEE to reduce dust during the dumping operation. There is an immediate need by the INEL Environmental Restoration remediation projects for demonstrations of conveyance systems as production retrieval activities may start by FY-96.

As required by the Comprehensive Environmental Response, Compensation, and Liability Act (CERCLA), alternative technologies must be considered to support the formal Record of Decision (ROD) concerning removal of buried waste. The primary criteria set forth by CERCLA for evaluation of technologies include (a) perform work faster to facilitate remediation schedules and shorten development 
_. . . . 


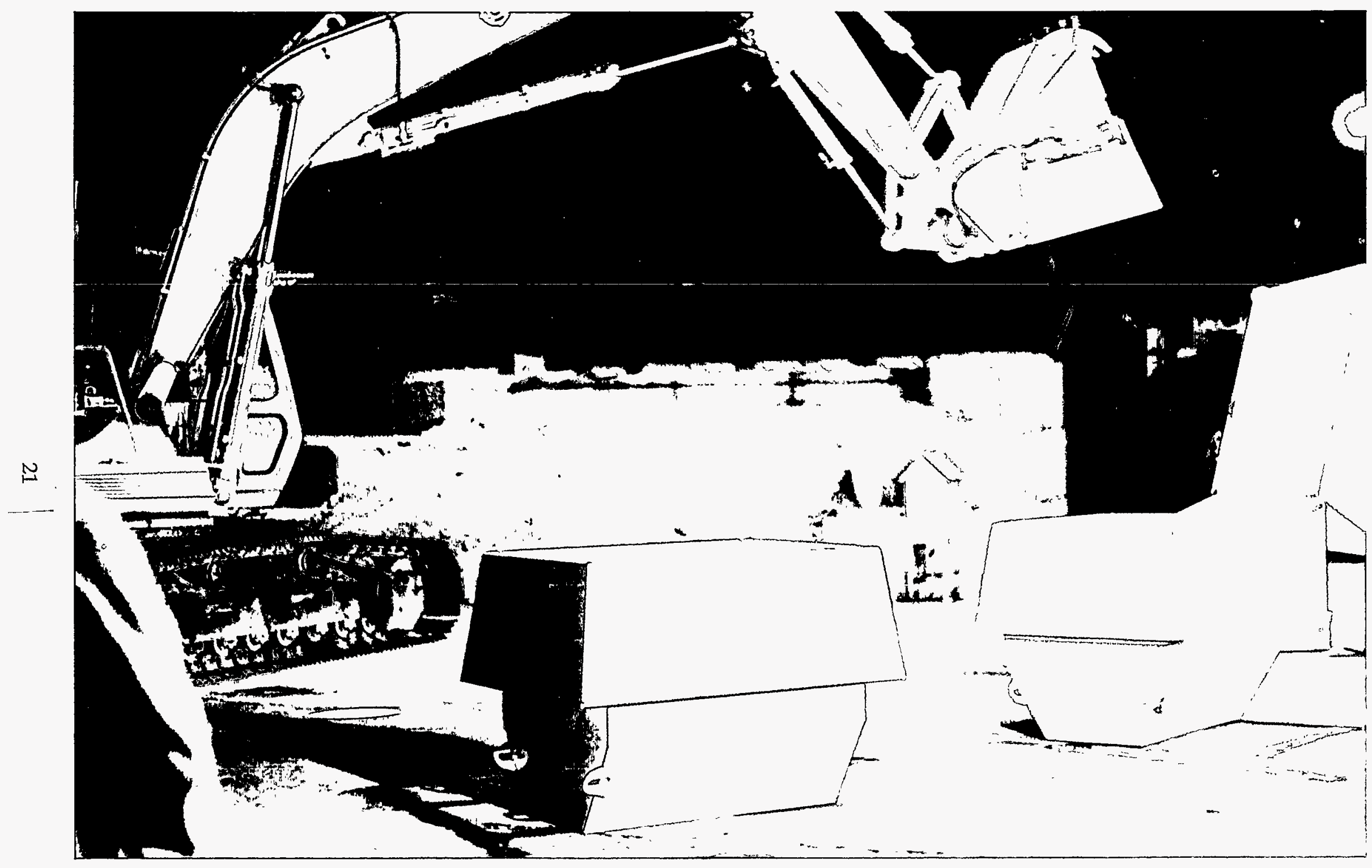

Figure 10. The Phase I test was performed inside a building where wind would not affect the dust and rare earth tracer measurements (INEL 94-1187-20-13A). 



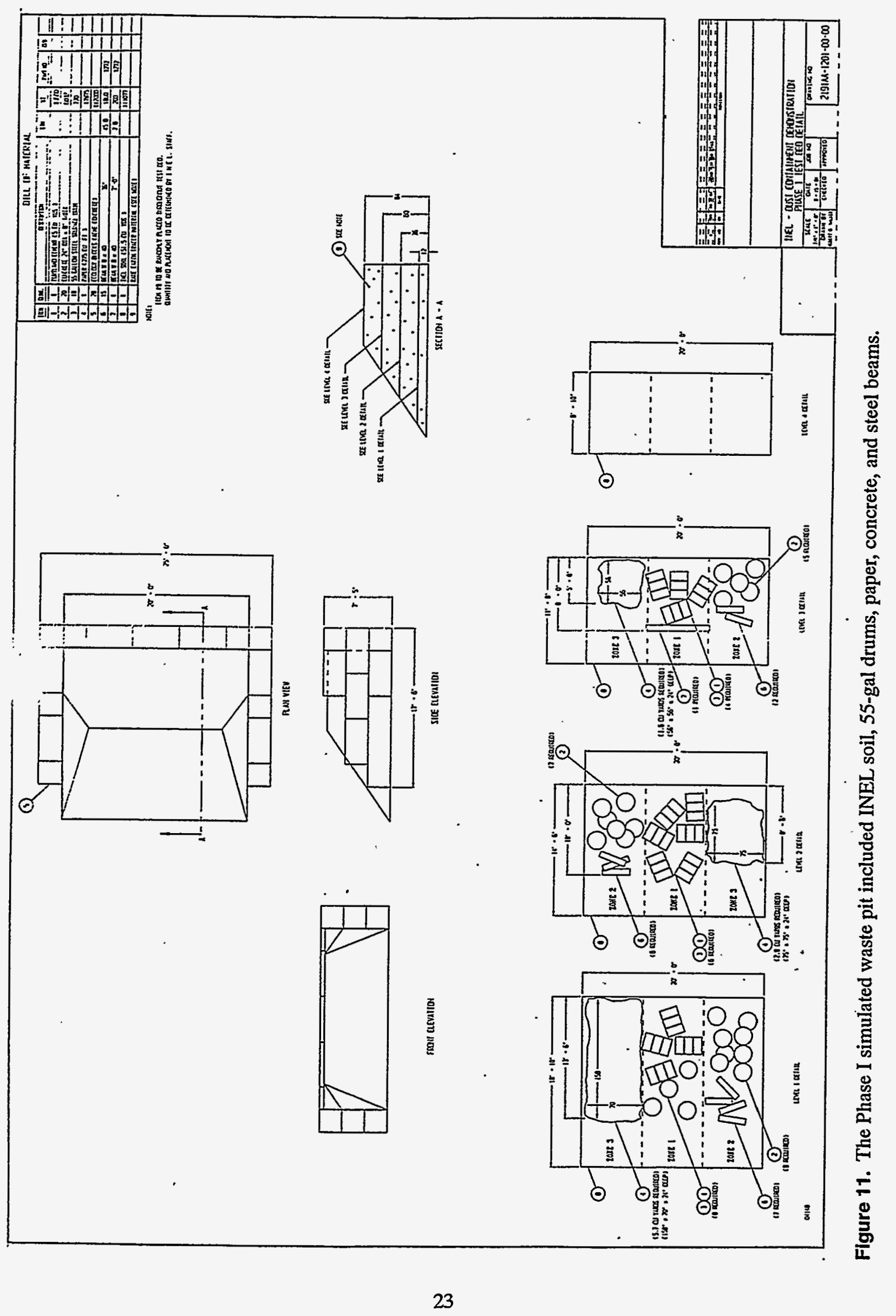




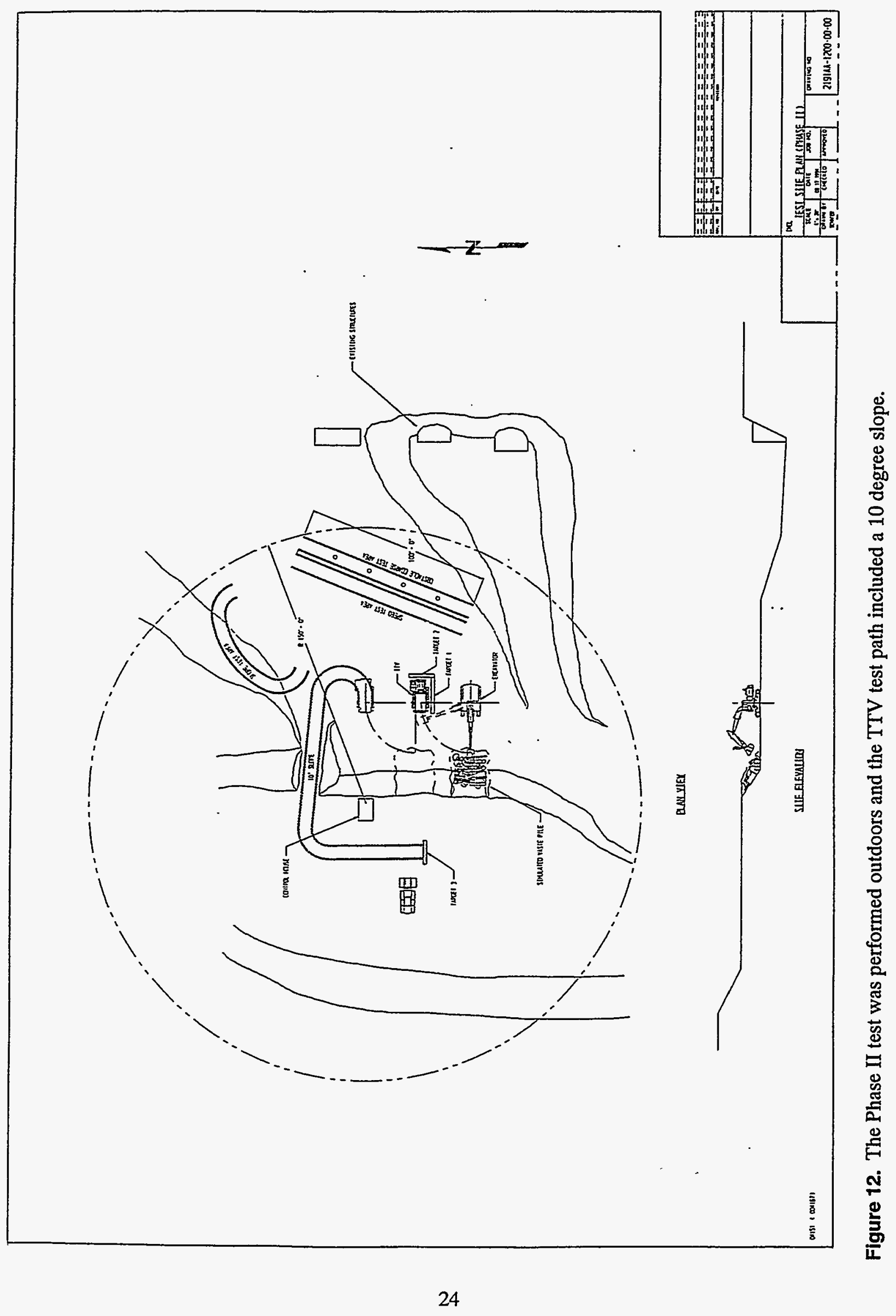


time, (b) use better technologies that reduce waste generated during remediation and allow for lower levels of decontamination, (c) reduce remediation costs, and (d) ensure that the technologies are safe by reducing personnel hazards, are in compliance with required laws and regulations, and improve environmental monitoring and assessment capabilities.

Table 1 depicts the various DOE sites where the IEE and remote conveyance system could be used to remediate DOE waste sites in order to assist with meeting these requirements.

Handling TRU waste during retrieval operations requires applying aggressive contamination control and personnel safety techniques, primarily because of the highly mobile nature of the TRU contaminants and the danger of fire and explosion from various interred hazardous materials: Remote waste retrieval and conveyance systems and dust free dumping will aid in the environmental restoration of DOE buried waste sites by lowering risk to workers and minimizing exposure to radiation and hazardous materials. Reducing operator fatigue and the possibility of errors should reduce risk to the environment. Also reducing dust that may contain airborne contaminants should reduce the risks to workers and the environment.

Table 1. Potential DOE application areas for the remote excavator/IEE and conveyance systems.

Potential application areas: BWL/ER/WM ${ }^{\mathrm{a}}$

\begin{tabular}{|c|c|c|c|c|c|c|c|c|c|c|}
\hline \multirow[b]{2}{*}{ Technology } & \multicolumn{10}{|c|}{ DOE Sites ${ }^{b}$} \\
\hline & AL & $\mathrm{CH}$ & FN & $\mathrm{ID}$ & NV & OR & $\underline{R F}$ & RL & SF & SR \\
\hline Waste Conveyance & $\begin{array}{l}\text { DRAINS } \\
\text { MDA } \\
\text { RSITE } \\
\text { SLF } \\
\text { PX-1 } \\
\text { PX-7 } \\
\text { PX-13 } \\
\text { DRAINS }\end{array}$ & East-5 & $\begin{array}{l}\text { OU1 } \\
\text { OU2-1 }\end{array}$ & $\begin{array}{l}1-3,6 \\
3-11 \\
7-10,13 \\
10 \text {-soils }\end{array}$ & $\begin{array}{l}\text { SIW1 } \\
\text { SIW2 }\end{array}$ & $\begin{array}{l}\text { WAG } 5 \\
\text { Bear Creek } \\
\text { (OU-1) }\end{array}$ & $\begin{array}{l}\text { OU-1S } \\
\text { OU-2S } \\
\text { OU-2B } \\
\text { OU-5/6 } \\
\text { OU-9P } \\
\text { OU-1S }\end{array}$ & $200-17$ & LRHR-S7 & ORWGB \\
\hline & $\begin{array}{l}\text { MDA } \\
\text { RSITE } \\
\text { SLF } \\
\text { PX-1 } \\
\text { PX-7 } \\
\text { PX-13 }\end{array}$ & & OU2-1 & $\begin{array}{l}3-11 \\
7-10,13 \\
10-\text { soils }\end{array}$ & SIW2 & $\begin{array}{l}\text { Bear Creek } \\
\text { (OU-1) }\end{array}$ & $\begin{array}{l}\text { OU-2S } \\
\text { OU-2B } \\
\text { OU-5/6 } \\
\text { OU-9P }\end{array}$ & & & \\
\hline \multicolumn{11}{|c|}{ a. Buried Waste Integrated Demonstration Environmental Restoration Waste Management. } \\
\hline $\begin{array}{l}\text { b. DOE Sites } \\
\text { AL-Albuquerq } \\
\text { CH-Chicago } \\
\text { FN-Fernald } \\
\text { ID-Idaho } \\
\text { NV-Nevada } \\
\text { OR-Oak Ridge } \\
\text { RF-Rocky Flat } \\
\text { RL-Richland } \\
\text { SF-San Francis } \\
\text { SR-Savannah R }\end{array}$ & & & & & 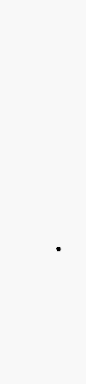 & & & & & \\
\hline
\end{tabular}




\section{TEST OBJECTIVES}

\subsection{Phase I Demonstration Test and Data Quality Objectives}

Phase I consisted of a full-scale demonstration to assess the ability of the IEE to control dust generation and the potential spread of contamination during dumping operations.

The following specific objectives for Phase I of the demonstration are reiterated from the Test Plan. ${ }^{4}$ Note that the REMEX was not specifically addressed in the test objectives because it was offered as a value added feature that was not part of the original scope of work.

\subsubsection{Dust Measurements}

Test Objective: Demonstrate the ability of the equipment and processes to control the generation of dust during dumping operations to a level of $1.1 \times 10^{-4} \mathrm{~g} / \mathrm{L}$ of air above background levels. This level $\left(1.1 \times 10^{-4} \mathrm{~g} / \mathrm{L}\right)$ represents a contaminated dust level that will allow personnel wearing bubble suits to enter a retrieval zone with an airborne source term of $<10 \mathrm{nCi} / \mathrm{g}$. The derivation of the dust level can be found in Appendix C.

Data Quality Objective: Determine with a $95 \%$ confidence level if dust at $1.1 \times 10^{-4} \mathrm{~g} / \mathrm{L}$ of air above background levels was detected.

Uncertainty: The following total uncertainty specifications apply for the measurements:

- Airflow measurements through the particulate samplers: Maintained within $10 \%$ of calibrated value.

The $10 \%$ flow total uncertainty was derived from the accuracy of calibration of the instruments used in this test for particulate air sampling.

- Mass measurements of the air sampler filters within $0.0001 \mathrm{~g}$.

The 0.0001-g total uncertainty was derived from the accuracy of calibration of the instruments used in this test for weighing the particulate air sample filters.

- Soil moisture measurements: Uncertainty will be reported with the data.

Soil moisture is determined by weighing and drying a soil sample. Soil samples are weighed to the nearest $0.0001 \mathrm{~g}$ before and after desiccation.

\subsubsection{Tracer Measurement}

Test Objective: Demonstrate the ability of the equipment and processes to control the spread of contamination.

Data Quality Objective: Determine with a 95\% confidence level if rare earth tracer (praseodymium) was detected above background levels. 
Uncertainty: The following total uncertainty specifications apply for the measurements:

- Airflow measurements through the particulate samplers: Maintained within $10 \%$ of calibrated value.

The $10 \%$ flow total uncertainty was derived from the accuracy of calibration of the instruments used in this test for particulate air sampling.

- Mass measurements of the air sampler filters within $0.0001 \mathrm{~g}$.

The $0.0001-g$ total uncertainty was derived from the accuracy of calibration of the instruments used in this test for weighing the particulate air sample filters.

- Soil moisture measurements: Uncertainty will be reported with the data.

Soil moisture is determined by weighing and drying a soil sample. Soil samples are weighed to the nearest $0.0001 \mathrm{~g}$ before and after desiccation.

- Analytical variation for the rare earth tracer praseodymium:

Instrument detection limit $-0.032 \mu \mathrm{g} / \mathrm{L}$

Standard concentration $=0.200 \mu \mathrm{g} / \mathrm{L}$

Measured 7 aliquots ( 3 reps each) on 3 separate days:

$\begin{array}{lll}\text { Average value } & =0.2424 \mu \mathrm{g} / \mathrm{L} \\ \% \text { Recovery } & =121.2 \% \\ \text { Standard deviation } & =0.0107 \mu \mathrm{g} / \mathrm{L}\end{array}$

Method accuracy and variation example for praseodymium previously used as a surrogate spike:

HI-VOL air sampler (1 set of digestions and analysis)

Actual

$=100 \mu \mathrm{g} / \mathrm{L}$

Average

$=104.77 \mu \mathrm{g} / \mathrm{L}$

Standard deviation

$=3.896 \mu \mathrm{g} / \mathrm{L}$

Minimum

$=98.87 \mu \mathrm{g} / \mathrm{L}$

Maximum

$=110.95 \mu \mathrm{g} / \mathrm{L}$

Note: Analytical variation is the standard instrument variation for the rare earth tracer praseodymium using the INEL Inductively Coupled Plasma Mass Spectrometry (ICP-MS) equipment. The specific analysis detection limits for the data gathered during this test are given in Appendix D. 
Previous studies performed at the INEL have shown that rare earth tracer can act as a surrogate for plutonium to evaluate the spread of contamination. ${ }^{5}$

The rare earth tracer, praseodymium, was chosen for several reasons: (a) of the lanthanide series rare earth elements, praseodymium's typical earth crust level (parts per million) is only exceeded by cerium and neodymium, making it relatively easy to obtain; (b) for the analytical measurements, there are no interfering ions for ${ }^{141} \mathrm{Pr}$; and (c) praseodymium is relatively inexpensive compared to other rare earth tracers.

The praseodymium was spiked in the soil and simulated waste at approximately 5 times background level. Natural background level for praseodymium in the earth's crust is approximately $6 \mathrm{ppm}$. To achieve a level of $30 \mathrm{ppm}(\mu \mathrm{g} / \mathrm{g}$ ), a total of $2 \mathrm{~kg}$ of praseodymium were added to the soil/waste mixture. The following is the calculation for the praseodymium spike:

Total soil $=\quad 50 \mathrm{yd}^{3}$

Total waste

$=16 \mathrm{yd}^{3}$

Total waste volume $=66 \mathrm{yd}^{3}$

Consider only the soil volume for the purposes of calculating the rare earth tracer spike:

$\left(50 \mathrm{yd}^{3}\right) \times\left(27 \mathrm{ft}^{3} / \mathrm{yd}^{3}\right) \times\left(105 \mathrm{lb} / \mathrm{ft}^{3}\right) \times(454 \mathrm{~g} / \mathrm{lb})=64,354,500 \mathrm{~g}$ of soil.

To achieve $30 \mu \mathrm{g} / \mathrm{g}$,

$(64,354,500 \mathrm{~g}$ of soil $) \times\left(30 \times 10^{-6} \mathrm{~g}(\mathrm{Pr}) / \mathrm{g}\right.$ of soil $)=1,930 \mathrm{~g}(\mathrm{Pr})$

The praseodymium was purchased in even quantities, so $2,000 \mathrm{~g}$ was used to spike the soil/waste mixture.

A "mini-test" was run to determine if the soil spike at 5 times background could be easily detected on the filters (i.e., was high enough to be detected above background) and to determine if the assumption that a 1:1 correlation between dust and tracer was reasonable. An EG\&G Idaho, Inc. letter report AMS-23-94a was issued to document this test. Summary results indicated that (probably due to the micron sized particles of praseodymium) the praseodymium was detected at levels approximately 100 times background. This indicates that the $1: 1$ correlation assumption is conservative and the 30 -ppm spike level should be sufficient to provide detection on filters that collect an adequate amount of dust.

\subsubsection{Operational Evaluation}

Test Objective: Evaluate the capability of the system.

Data Quality Objective: Observe the operations of the IEE system and record information concerning: spillage, ability to handle the waste forms, visual estimate of dust generation, reliability,

a. A. M. Smith, Basis for Dust Level, EG\&G Idaho, Inc., Letter Report, AMS-23-94. 
ease of use, ease of maintenance, and any other operational parameters that could be used to determine the system's capability. Documentation shall include photographs, videotape, and logbook entries. Specific items to document include

- Volume of waste removed per excavation cycle

- Waste spillage volume measurements

- Observations on system excavation effectiveness and ability to handle waste forms

- Visual observations of dust generated during the complete excavation and dumping cycle

- Ease of use of the end effector system, including ITM attachment/detachment to end effector and ITM placement into transport container

- Anomalous conditions

- Overall reliability of end effector system

- Frequency and type of maintenance required during the demonstration, both scheduled and unscheduled

- Length of time required for maintenance activities.

Uncertainty: The following total uncertainty specifications apply for the measurements:

- The volume of waste removed per cycle: $10 \%$ of end effector capacity.

The $10 \%$ total uncertainty was derived using engineering judgment. The excavator bucket is capable of holding $1.4 \mathrm{yd}^{3}$. By allowing field personnel to judge the fullness of the bucket, it was determined that this was an achievable goal and would adequately satisfy the data quality objective.

- Waste spillage volume measurements: $0.0002 \mathrm{yd}^{3} / \mathrm{cycle}$.

The $0.0002 \mathrm{yd}^{3} /$ cycle was derived using engineering judgment. It is based on the assumption that measurements of spillage volume can be made to this accuracy by following the specified spillage data gathering procedure. This procedure involves gathering the spilled waste and placing it into a calibrated container.

\subsubsection{Effectiveness on Various Waste Forms}

Test Objective: Determine the capability of the end effector to handle the various objects that could be encountered in an actual operation.

Data Quality Objective: Document the various waste forms encountered and the ability of the system to move these objects through the entire excavation and transport process using photographs, 
videotape, and logbook entries. Document the affect the waste type has on spillage, equipment jams or failure, and on the rate of retrieval for the entire system using photographs, videotape, and logbook entries.

Uncertainty: Not applicable.

\subsubsection{Overall Phase I Demonstration Success}

This phase of the demonstration can be deemed a success if sufficient data and operations experience are gained to assess the capability of this technology to accomplish its goals and if Environmental Restoration is provided with data to perform the feasibility studies. This determination will be made based on the following:

- Collection of sufficient quantitative data to make a determination on the spread of contamination resulting from the application of the equipment and processes

- Collection of sufficient quantitative data to make a determination on the amount of dust generation resulting from the application of the equipment and processes

- Observations regarding overall system performance including: spillage, ability to handle the waste forms, visual estimate of dust suppression, reliability, ease of use, ease of maintenance, and any other operational parameters made and recorded for future reference.

\subsection{Phase II Demonstration Test and Data Quality Objectives}

This phase consisted of a full-scale retrieval/conveyance demonstration. The demonstration included all elements of the REMEX, waste conveyance, and end effector system. The demonstration excavated, transferred, and conveyed simulated waste from the test arena to demonstrate the functionality of the system (e.g., maneuverability and retrieval rates).

The following specific objectives for Phase II of the demonstration are reiterated from the test plan. 4

\subsubsection{Throughput}

Test Objective: To measure the production rate for dumping and conveyance of waste from the digface based on use of the selected equipment and technologies. A goal of $80 \mathrm{yd}^{3} / \mathrm{day}$ in an 8-hour shift will be used for design of the demonstration system. Each conveyed waste container or end effector will be allowed to hold a maximum of approximately $1.4 \mathrm{yd}^{3}$ of simulated buried waste in the form of waste mixed with soil. Some large objects or intact waste boxes may be larger than $1.4 \mathrm{yd}^{3}$.

Data Quality Objective: Document the rate of waste removal and transport as performed during the demonstration by measuring the time of actual excavation operations and number of cubic yards of soil removed and conveyed.

Uncertainty: The following total uncertainty specifications apply for the measurements:

- The volume of waste removed and transported per cycle: $10 \%$ of end effector capacity. 
The $10 \%$ total uncertainty was derived using engineering judgment. The excavator bucket is capable of holding $1.4 \mathrm{yd}^{3}$. By allowing field personnel to judge the fullness of the bucket, it was determined that this was an achievable goal and would adequately satisfy the data quality objective.

- The time required: .25 minute per cycle.

The .25-minute total uncertainty was derived using engineering judgment. This time accuracy was selected because it is easy to measure and provides sufficient accuracy to satisfy the data quality objective.

\subsubsection{Effectiveness on Large Objects}

Test Objective: Determine the capability of the end effector to handle the various typical selected large (large being greater than 55-gal drum) waste forms that could be encountered in an actual operation.

Data Quality Objective: Document the type of large objects encountered and the ability of the system to selectively retrieve, lift, and set these objects to the side of the excavation area using photographs, videotape, and logbook entries. Document the number and type of large objects dropped and the amount of slip for objects successfully moved using photographs, videotape, and logbook entries. Estimate and document in logbook entries the percentage that operator skill and/or experience has on the ability of the equipment to perform these functions.

Uncertainty: Not applicable.

\subsubsection{Effectiveness on Various Waste Forms}

Test Objective: Determine the capability of the end effector to handle the various objects that could be encountered in an actual operation.

Data Quality Objective: Document the various waste forms encountered and the ability of the system to move these objects through the entire excavation and transport process using photographs, videotape, and logbook entries. Document the affect the waste type has on spillage, equipment jams or failure, and on the rate of retrieval for the entire system using photographs, videotape, and logbook entries.

Uncertainty: Not applicable.

\subsubsection{Effectiveness of Telerobotic Control System}

Demonstrate the effectiveness of the remote control system from measurements, observations, and operator interviews during demonstration retrieval operations.

Data Quality Objective: Document the capabilities of the remote control system (e.g., range and signal strength) and the ability of the system to effectively control the remote conveyance system by photographs, videotape, logbook entries, and operator interview records. 
Some data are qualitative by nature but crucial to understanding the effectiveness of the remote control system. In particular, the ergonomics (human factors) design of the operator control station was documented. Each operator was interviewed to determine his/her opinion on the following:

- Is the control joystick easily within reach, does it have appropriate "feel," and are the corresponding motions of the vehicle intuitive

- Are the status indicators (lights, LCD display, and audio signal) easily understood and are warning indicators noticeable

- Are the lid and latch control switches easily within reach and adequate for the purpose

- Is the view provided by the TTV-mounted cameras adequate for the purpose of teleoperation

- Does eye strain result from continuous watching of the video monitor

- Are the camera pan, tilt, zoom, and focus controls easily within reach and intuitive.

Measure the capabilities of the remote control system by recording the following data:

- Telemetry system range: With diagnostic monitors connected to the vehicle control system and the operator station, increase the distance separating the vehicle from the base station and measure the ranges corresponding to successful telemetry throughput of $95 \%, 90 \%, 80 \%$, and $70 \%$. Report transmitter specifications, power, antenna design, etc. (This test was modified; see Section 3.2.)

TV system range: Using a spectrum analyzer, measure the television signal strength (in $\mathrm{dB}$ ) as a function of line-of-sight range from the base station to the TTV. Report transmitter specifications, power, antenna design, etc. (This test was deleted; see Section 3.2.)

- Travel capability: To measure the effectiveness of the remote control joystick, verify maximum speed of the vehicle by time-to-distance measurements. Measure the smallest distance the TTV can move repeatedly in a straight line.

- Vision capability: Record by logbook entries the effective viewing range of the forward and aft cameras.

- Time/motion studies: To make comparisons between manual, telerobotic, and self-guided (future) control of the equipment, time data shall be gathered with respect to the following parameters:

- Connecting and disconnecting the ITM from the excavator.

- Transport time between the loading station and waste receiving station over a fixed path of known length. 
- Reliability: Record how many loading/unloading cycles can be accomplished before humàn intervention (other than normal teleoperation) for control or other problems is required. Record the reason for any human intervention.

Because the operational safety of the TTV is of the utmost importance, the following were documented:

- Test and document by logbook entries the function of the emergency kill switch on the control station and conveyance vehicle.

- Test and document by logbook entries, photographs, and videotape the function of the collision avoidance system (using obstacles of varying size and position), the motion alarm system, the motion indicator light, and any other safety features of the design.

- Anomalies such as the number of times the conveyance system responds differently than expected to the sent signal, the number of times the remote conveyance system receives an unintended signal, and/or the ability of the control system to regain control if such a malfunction occurs.

- Any other system malfunctions.

- Safeguards designed to prevent human error.

- Operator errors, the response of the system to such errors, and the procedures necessary to correct them.

Uncertainty: Not applicable or will be reported with the data.

\subsubsection{Maneuverability and Capability on Rugged Terrain}

Test Objective: Determine the maneuverability and ability of the conveyance system to operate on rugged terrain encountered during demonstration retrieval operations. Determine the precision and accuracy of the guidance system by measuring the difference between the expected and actual location of the TTV.

Data Quality Objective: Document the maneuverability on rugged terrain of the remote conveyance system used in the demonstration by direct measurements and photographic evidence. Determine with a 95\% confidence level that for any specified target location, the average placement of the TTV was within 1 radial foot of that target location. Document the level of variability in the terrain and the affect these variations have on vehicle maneuverability by direct measurements and photographic evidence.

Uncertainty: The following total uncertainty specifications apply for the measurements:

- Location of reference point on TTV to target location: 0.25 in.

The 0.25 -in. uncertainty was derived from engineering judgment. The uncertainties involved with this distance include the fact that the measurement using a tape measure for the location of the TTV when parked at the excavator or receiving station ignores any contribution from 
differences in elevation between reference marks on the TTV, excavator, and receiving station. However, the specified test site is relatively flat and the error introduced by ignoring the difference in elevation is negligible. Therefore, a 0.25 -in. uncertainty was selected because it is easy to measure and would adequately satisfy the data quality objective.

- $\quad$ Slope of TTV path on simulated hill: $10 \%$.

The $10 \%$ slope uncertainty was derived based on the assumption that a surveyor's transit and stadia will be used to survey the simulated hill. The hill topography will be documented using this equipment.

\subsubsection{Transuranic Environment Evaluation}

Estimate the capability of the system for operation in a TRU-contaminated environment (including deployment, maintenance, repairability, reliability, and operating cost).

Data Quality Objective: Determine the capability of the system to be used in a TRU contaminated environment by documenting through logbook entries, photographs, and videotape the following items:

- Ruggedness of the equipment

- Frequency and type of maintenance required during the demonstration, both scheduled and unscheduled

- Length of time required for maintenance activities

- $\quad$ Ease and speed of deployment

- Ability of the system to easily correct for control system errors and/or operator errors.

Uncertainty: Not applicable.

\subsubsection{System Integration}

Test Objective: Demonstrate the smooth integration of the IEE and remote conveyance system.

Data Quality Objective: Document the level of integration of the remote conveyance and IEE system and the affect that they have on the smooth operation of the system using photographs, videotape, and logbook entries. Specific items to document include

- How well the remote conveyance system receives or picks up the IEE or transport container

- How well the placement system (i.e., targets) works

- To what extent system integration (e.g., fit and smooth operation) was built into the design of the two systems (i.e., are the designs concurrent and integral to each other so that overall system performance is improved).

Uncertainty: Not applicable. 


\section{EXPERIMENTAL DESIGN AND PROCEDURES}

During the demonstration, some of the test procedures were changed to accommodate the demonstration configuration and time limitations. The changes are detailed in this section, and the corrected test procedures are in Appendix E.

\subsection{Test Design and Methodology}

\subsubsection{Phase I Demonstration-Dust and Contamination Spread}

The Phase I demonstration consisted of a full-scale test to assess the ability of the IEE to control dust generation and the potential spread of contamination during dumping operations. The test was performed at RAHCO's facility under controlled environmental conditions (such as would occur inside a retrieval containment structure). The demonstration included both digging and dumping operations; however, the primary objective was to test the ability of the system to reduce the generation and spread of dust during the dumping operation.

This demonstration consisted of digging and dumping dry, INEL soils mixed with simulated waste using the RAHCO-designed IEE. The demonstration was performed in an indoor facility where environmental conditions (especially air circulation) were controlled so that effective dust and rare earth tracer measurements could be taken. Figure 13 illustrates the general arrangement for the Phase I test. The digging and dumping operations were separated so that the effects of dust generated from the digging on the dumping portion of the test could be accounted for (because the dust generated during dumping was of interest). The subcontractor elected to develop an end effector instead of a dust free dumping system, so the test consisted of the digging phase and whatever actions were required to deposit the end effector for the conveyance system to transport. The demonstration consisted of a series of digging and dumping actions from a pile of approximately $50 \mathrm{yd}^{3}$ of mixed soil and simulated waste. The soil and waste were spiked with a rare earth tracer (a rare, nonhazardous, nonradioactive element) to provide an easy method to analyze and evaluate samples. Dust measurements were made by EG\&G Idaho. The samples were analyzed to determine how the generation and suspension of dust could be minimized during the dumping operations. These results are given in Section 7.

\subsubsection{Phase II Demonstration-Retrieval and Conveyance}

The Phase II demonstration consisted of a full-scale retrieval of simulated buried waste using the TTV, IEE, and REMEX. The purpose of this phase was to assess the ability of the end effector, waste conveyance system, and REMEX to effectively handle, transfer, and transport the waste from the digface. This phase of the demonstration was also performed at RAHCO's facility, but was performed outdoors. The demonstration included all elements of the waste conveyance and end effector systems. The demonstration excavated, dumped, and conveyed simulated waste from the test area to demonstrate the functionality of the system (e.g., maneuverability and retrieval rates). Figure 14 illustrates the general arrangement for the Phase II test. During the retrieval activities, observations and measurements were taken to determine how efficiently the equipment excavated the waste, worked around or handled large objects, and conveyed the waste from the excavation site. Assessment of the conveyance system's maneuverability, precision, accuracy, and flexibility was made. Observations were also made of the remote control system, system integration between the remote conveyance system and IEE, and the effect 


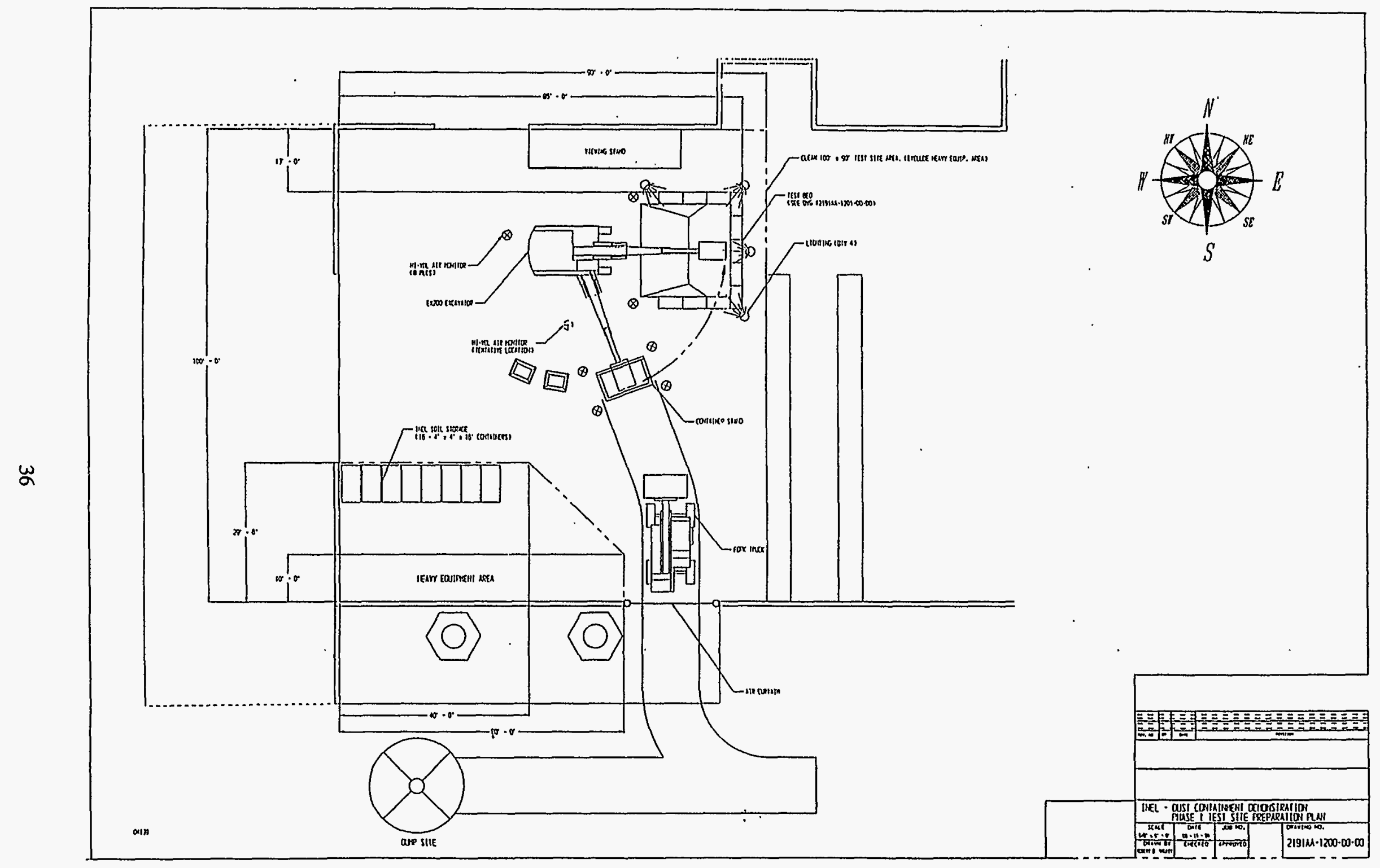

Figure 13. The Phase I test was conducted in an enclosed structure to ensure controlled environmental conditions. The primary purpose of the Phase I test was to determine the amount of dust generated during waste dumping and level of potential contamination spread. 


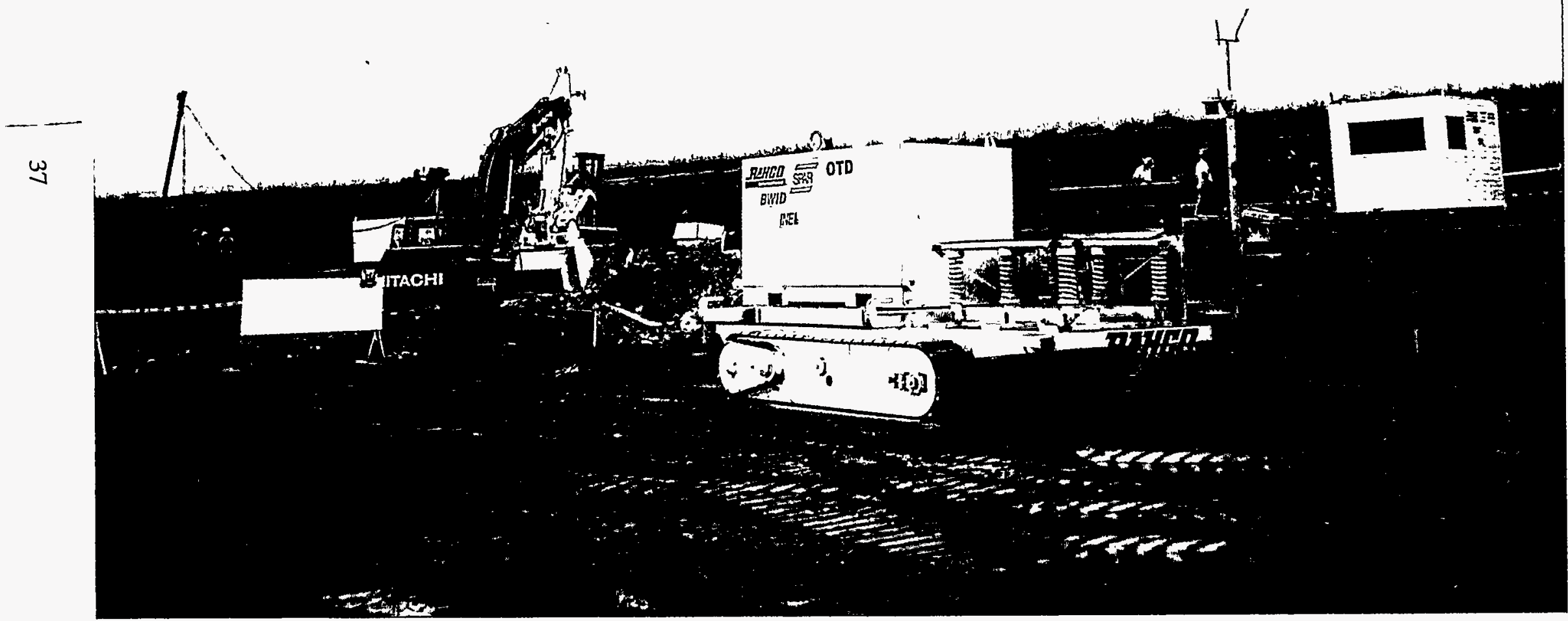

Figure 14. The Phase II test involved exhumation of simulated buried TRU waste. The primary purpose of the Phase II test was to demonstrate the remote conveyance system and examine the integration between the conveyance system, IEE, and REMEX (INEL 94-1187-8-9). 
- 
of system integration on overall system performance. No dust measurements were made during this demonstration; however, qualitative data were gathered on the ability of the system to control dust generation during these operations. The test results are given in Section 7.

\subsubsection{Equipment and Instruments}

RAHCO provided all utilities and services, such as water, power, phone, and sanitary, at the work location. RAHCO supplied forklifts to move the transfer containers or receptacles as necessary during the demonstration. RAHCO was responsible for all transporting, fuel, maintenance, and operation of all machines. SPAR working in conjunction with RSI Research, Ltd., provided the REMEX and TTV sensor, vision, and control systems.

RAHCO was also responsible for providing operators for the machines. The operators designated for the excavator at the start of the test were available throughout the duration of the demonstration. This ensured continuity of operation and consistency of comments and feedback.

RAHCO provided approximately $16 \mathrm{yd}^{3}$ of simulated waste forms in the following proportions:

- Paper/cloth-50\%

- 55-gal drums filled with dry Portland cement-30\%

- $\quad$ Metal-10\%

- Concrete/asphalt-10\%.

RAHCO also included a variety of large objects for the Phase II test: a pickup truck bed, $15 \mathrm{ft}$ lengths of carbon steel pipe of varying diameter and schedule (maximum diameter $16 \mathrm{in}$. and maximum schedule 40), $15 \mathrm{ft}$ lengths of carbon steel structural shapes of varying weight (maximum $40 \mathrm{lb} / \mathrm{lineal}$ foot weight), and a maximum of $200 \mathrm{ft}$ of 1 -in. (or less) steel cable (in maximum 50 -ft lengths).

EG\&G Idaho provided approximately $43 \mathrm{yd}^{3}$ of dry INEL soil from the RWMC and dry lake bed areas. The soil was placed in closed $4 \times 4 \times 8$-ft transport boxes and shipped to RAHCO's testing site, where it was stored inside of the Phase I demonstration building. EG\&G Idaho provided the rare earth tracer (praseodymium) that was used to spike the simulated waste pit. Praseodymium is a nonradioactive, nonhazardous material. The HI-VOL particulate air samplers were also provided by EG\&G Idaho. EG\&G Idaho personnel assisted with the demonstration and data collection activities.

Prior to construction of the waste pit, background praseodymium levels were measured in the demonstration building. See Appendix $\mathrm{E}$ for the test procedure. The waste pit was constructed using the waste forms described in Section 3.1.1, praseodymium, and INEL soil. Details of pit construction can be found in Figure 11. 


\subsection{Deviations from Test Plan}

Deviations from the test plan $^{4}$ were noted in the master copy of the test plan and approved by the RAHCO project engineer and the LITCO principal investigator. These changes included the following:

- TV system range and signal strength were not measured using a spectrum analyzer because the low power TV system selected may have made it difficult to obtain valid data with a spectrum analyzer. In order to meet Federal Communications Commission (FCC) regulations, the TV system used was a low power, shorter range system than the one originally proposed. Instead, observations on the TV signal strength were made during the demonstration, during maneuverability testing, and during the TTV telemetry range test. The telemetry range test was conducted by starting the TTV at the remote control station and then driving a distance of $500 \mathrm{ft}$. At set intervals of 10 seconds, the distance traveled and number of data packets received were recorded. Since the TTV sends signals at a continuous frequency, the number of lost information packets can be inferred from the number of good signals received. As a double check, the number of errors (corrupt or unreadable data packets received) was monitored as the vehicle returned to the control station.

- The test plan specified that $66 \mathrm{yd}^{3}$ of soil and waste would be moved during the Phase I test. Due to a reduced amount of INEL soil being shipped to RAHCO (43 instead of $50 \mathrm{yd}^{3}$ ), the actual amount of waste and soil was approximately $59 \mathrm{yd}^{3}$.

- Although the test plan specified no line-of-sight person to assist with remote operation, during the early stages of Phase II testing, the video signals were inadequate to allow for TTV operation without aid. A line-of-sight person provided radio guidance to the TTV operator during the first stages of Phase II. Following repositioning of one of the cameras, and learning that the excavator cameras could assist the TTV operator, the radio communications regarding directions were discontinued. In addition, during the maneuverability test, the video signal disappeared at some locations so radio contact was used to ensure safe operation.

- The function of the emergency kill switch on the control station supervisor's belt module, and conveyance vehicle was tested during performance testing (prior to the start of the demonstration) rather than during the demonstration. This was done to ensure that the emergency kill switch would work before starting the demonstration.

- A detailed test procedure that was written by RAHCO and a HI-VOL air monitor test procedure, written by EG\&G Idaho (see Appendix E) were inserted into the master copy of the test plan $^{4}$ before starting the demonstration. These procedures were dependent on the details of the test layout, which was not available at the time of the test plan release.

- The volume of waste moved during Phase II was approximately $50 \mathrm{yd}^{3}$ rather than 300 to $400 \mathrm{yd}^{3}$ as anticipated in the test plan. This was because the increased path length and limited TTV engine horsepower limited the volume that could be moved, and the test schedule required completion by September 30, 1994. 
- The collision avoidance test specified in the test plan was not completed because of crosstalk between the ultrasonic sensors.

- The Portland cement was dry rather than sludge because of a miscommunication between the INEL and RAH CO.

\subsection{Deviations from Test Procedure}

Deviations from the test procedure were noted in the master copy of the test plan ${ }^{4}$ and approved by the RAHCO project engineer and LITCO principal investigator. An updated copy of the actual test procedure is included in Appendix E. In summary, the changes to the test procedure included the following:

Phase I:

- Background readings were taken for 60 minutes rather than 30 minutes to ensure an adequate reading.

- Ten $\mathrm{yd}^{3}$ per test were moved rather than 13 to $15 \mathrm{yd}^{3}$ to limit the amount of waste moved so that an adequate number of test cycles could be conducted.

- Each test ran for 1 to 2 hours rather than 3 to 4 hours to limit exposure to the diesel exhaust and limit the amount of waste moved so an adequate number of test cycles could be conducted.

- After the first test, the WTC was not used to allow for easier ITM dumping operations outside of the test area. The ITMs were moved by forklifts directly without being placed in the WTC.

- Spillage was estimated visually because there was so little spillage that placing it in a calibrated container and measuring it was not feasible.

- The level of rare earth tracer spiking was more than the amount specified in the test plan because the amount of INEL soil was reduced. The test plan specified spiking at $30 \mu \mathrm{g} / \mathrm{g}$, and the actual test spiking was at $36 \mu \mathrm{g} / \mathrm{g}$.

Phase II:

- Speed testing, maneuverability, turning radius, and ability to detect obstacles were all done as a part of performance testing rather than during each cycle of the demonstration to optimize throughput during the actual waste removal and transport and to obtain an accurate cycle time readings.

- The TTV did not mark its path of travel because there was not enough time to measure its ability to follow the path for each cycle.

- The ITMs, rather than the transport containers, were removed from the TTV during each cycle to facilitate the demonstration. 


\section{QUALITY ASSURANCE}

The Quality Level for this project was Quality Level 3, as defined in the EG\&G Idaho Quality Manual. ${ }^{6}$ All work performed during testing adhered to the EG\&G Idaho Quality Manual and to RAHCO's Quality Assurance Program. This includes documentation of all aspects of the project from engineering through customer sign off.

\subsection{Quality Control Methods and Procedures}

\subsubsection{Measures Taken}

EG\&G Idaho procedures for the logbook and data management were followed during the tests. Data in the logbook were recorded in ink and signed by the recorder and an independent verification person.

All testing was performed in accordance with an approved test plan, approved test procedures, and with qualified personnel (as defined in the procedures). The test procedures specified sensor and measurement systems to be used (e.g., model number, make, and calibration). Measurement uncertainty for data taken against the test objectives was documented.

The particulate air samplers were positioned at several locations around the test area. The preweighed particulate filters were removed from the petri dishes, placed in the filter holder, and the retaining ring was attached. The sampler filter was handled using tweezers to minimize contamination of the filter from moisture or natural skin oils from the hand. The filter number was noted in the logbook along with the sampler location and number. After the test run was completed, the particulate filters were removed from the air monitors using the same procedures that were used to install them and placed in their original petri dishes. The filters were kept together and protected from damage or contamination. A total of five complete test runs were done for Phase I of the demonstration. The test used 11, 4-in. filters as follows:

Each test used

3 filters for background measurements +8 filters for test samples, equaling 11 filters for each test run:

$11 \times 5=55$ total test filters

Laboratory analysis used:

8 filters for laboratory blanks for the entire analysis

5 filters for laboratory spikes

$$
55+13=68 \text { total filters }
$$

Two extra sets (total of 30 filters) were prepared as backup for use if a test had to be scrubbed for some reason. The back up filters were not used.

Routine monitoring was performed during the time that the HI-VOL air samplers were operational in the field. During this check, the person ensured that each sampler was operational and running within operational limits of the flow. In addition, the person conducted a routine safety inspection of the 
equipment and general area to ensure that no unsafe conditions occurred. Any changes made to the sampler were noted in the field logbook. Data recorded included manometer readings, temperature, barometric pressure, date, time, and record of action taken.

Data quality was verified using peer review by a person knowledgeable of data, assumptions, methodology, and individual measurement analysis to verify that data were within the performance specifications detailed in the test plan.

Quality assurance of samples collected was verified using several methods. All filters used for sampling were weighed on a balance having a current calibration certification from the EG\&G Idaho Standards and Calibrations Laboratory. HI-VOL air monitors had current calibrations and calibration data from the Standards and Calibrations Laboratory before use except as noted below. The calibration information was not referenced in the project logbook per the test plan but was kept on separate sheets and will be kept in the project file. See Appendix F for a summary of the calibration data. Note that the HI-VOL samplers are really just correlated and not actually calibrated because the output is a performance characteristic curve describing the function of each HI-VOL rather than an actual calibration to a standard.. The calibration procedure provides performance characteristic curves regarding the airflow at various motor speeds. Because the motors compensate to keep airflow constant as the filter becomes loaded, the motor speeds are somewhat representative of dust loading. Analytical results were verified by the use of prepared standards, use of duplicate samples, and submission of blanks with each ICP run, not with each sample set as stated in the test plan. ${ }^{4}$ The standards, duplicates, and blanks were not submitted as a blind to the analytic laboratory. Instead, blanks were submitted so that they could be used for spiking and reagent checks to ensure the quality assurance of the laboratory procedures.

The analytical quality control consisted of the preliminary interference study to determine if other interfering elements or polyatomic species present problems during ICP-MS analysis for the rare earth tracer. Included in each batch of samples was a minimum of four blank filters from the same batch used for sampling, two of which were spiked with known quantities of rare earth tracer and a reagent blank for a reagent impurity check.

\subsubsection{Effectiveness}

The logbooks were effective for noting progress, changes, and problems encountered. They served as the repository of all data. Review of the logbooks provided a check of the data. There were no consequences to keeping the calibration data separate from the logbook.

The changes in the number of blanks did not jeopardize the tests in any way. In fact, this change reduced the cost for analyzing the filters while preserving quality assurance. The stringent controls on the analytical methods contributed to quality assurance.

Two of the HI-VOLs required the measurement of new performance characteristic curves, or recalibration. HI-VOL \#4 was calibrated before the tests with the wrong filter. HI-VOL \#5 also required recalibration as the results from the sampler were erratic. HI-VOL \#5 was only used during the first test and was replaced by HI-VOL \#2. The recalibration of HI-VOL \#4 showed that this HI-VOL has an unusual airflow versus pressure curve, with an unusually high airflow trend. Although this behavior is 
unusual, it errs on the conservative side because it means that this HI-VOL had a higher airflow than the others and would have more dust as a result.

\subsection{Precision and Accuracy of Measured Data}

\subsubsection{Measures Taken}

Dust and Rare Earth Tracer Data: Instrument batch quality control samples consisted of an independent calibration standard check and continuing calibration standard and blank checks every ICP run. A post-digestion spike also was included in every ICP run. These quality control samples provided precision and accuracy estimates for the analytical methodology.

Maneuverability (Precision and Accuracy Data): The use of standard measuring tapes for the measurement of distances between reference marks provided data sufficient to meet the data quality objective.

Throughput: The inside of the ITMs was marked in increments of $1 / 2,3 / 4$, and $7 / 8$ full to assist with visual volume estimates.

\subsubsection{Results and Effectiveness}

The measurements were effective in determining the precision and accuracy. The maneuverability measurements were taken to the nearest $1 / 2 \mathrm{ft}$ rather than nearest $0.25 \mathrm{in}$. as reported in the test plan. This change was made due to the uncertainty of the positions of the fixed markers and the speed with which measurements needed to be taken to keep up with the vehicle motions. Docking measurements were taken to the nearest inch instead of the nearest 0.25 in. due to the variability in terrain.

\subsection{Change Control}

\subsubsection{Measures Taken}

Test Plan: Changes to test procedures were limited to those necessary to meet test objectives and were approved by the RAHCO project engineer and INEL principal investigator and were documented per the test plan. The effects of any changes on the test objectives were minimized to the greatest extent possible. The corrected test procedures are discussed in Section 3 and contained in Appendix E.

Performance Testing: Performance testing for maneuverability, speed, and telemetry (not including quantitative video signals) of the conveyance system that was to be performed before Phase II was actually performed during the Phase II testing. This change was a result of insufficient time to perform these tests before the start of the demonstration. A low power video system was used to meet FCC requirements, and therefore, the spectrum analyzer was not used to measure video signal strength.

Equipment Changes: Equipment changes occurred to correct problems and facilitate testing. These equipment changes are discussed in Section 7 since they affect the test results. 


\subsubsection{Results and Effectiveness}

Test Plan: Because of time limitations for performance testing and the demonstration configuration, certain test procedures required onsite modification. By allowing procedures to be redlined, the appropriate flexibility allowed testing to continue in a reasonable manner and within a reasonable schedule. The approvals of all changes ensured that changes were not random and would not jeopardize the test objectives. One change included operating the excavator manually for the last day of the tests. This allowed comparison of remote versus manual operations under the conditions described in Section 3.

Performance Testing: There were no consequences to performing the performance tests during the actual Phase II tests instead of before these tests.

\subsection{Internal Audits}

\subsubsection{Measures Taken}

BWID sent a quality assurance/quality control (QA/QC) Team Leader to the test site to review the tests. The review was held by BWID to ensure quality procedures were being followed. The reviewer inspected the readiness review checklists, performance test checklists, test procedures, data recording plans and sheets, and methods of data collection.

Other project reviewers included the BWID coordinator, BWID project manager, BWID technical liaison, and an BWID technical independent reviewer.

\subsubsection{Results and Effectiveness}

The BWID QA/QC reviewer reported that logbooks were being kept and proper procedures followed during the observed tests. The reviewer found that all testing was being performed in a satisfactory manner.

The other project reviewers expressed that the tests were successful and that all tests were being performed in a professional and safe manner.

\subsection{Subcontractor Quality Assurance Measures}

A complete QA program is in place and operating at RAHCO. This includes documentation of all aspects of the project from engineering through customer sign-off. Qualified people are involved at every level to form the QA inspection team.

Vendors and subcontractors are inspected and questioned on their QA programs. When vendor or raw material items are received, a receiving inspection form is filled out to ensure the item meets the contractual specification. Any defects or discrepancies are recorded, and the defective item is tagged and set aside to await disposition. If a subcontractor is used for overflow work, they are required to meet or exceed RAHCO quality standards as determined by the QA manager. Progress inspection visits ensure that quality is maintained to contract standards, schedule is maintained, and different machine subcomponents are integrated. All inspections are documented and filed. 
Engineering drawings and calculations are recorded and filed after undergoing a strict sign off procedure. As work progresses through the shop, the project engineer and shop worker as well as the QA department are responsible for quality. In-process inspections check and record all critical dimensions, weld quality, and paint. If there is a problem, a rejection notice is issued and a corrective action request is sent out. No item passes to the next phase until the corrective action request is signed and returned. The QA manager monitors all operations to ensure that approved engineering changes are incorporated. After the assembly inspection and the functional testing, a final inspection is performed and recorded.

Onsite testing inspection took place at RAHCO's test facility. During Phase I and Phase II testing, any modifications required were recorded and approved by the RAHCO project engineer and INEL principal investigator.

Packaging and shipping documentation is recorded by the shipping and receiving department. Vendor product and warranty information is assembled, recorded, and filed at RAHCO. All documentation of the QA program is directed through and filed under the supervision of the RAHCO quality manager. Any inquiries, corrections, or questions pertaining to these records are channeled through the RAHCO quality manager. 


\section{HEALTH AND SAFETY}

Safety is considered the highest priority for operations by the companies involved in this demonstration. DOE wants risks to workers to be removed to the greatest extent possible. Providing operators with the ability to control the equipment at a safe distance from the waste is the most reliable method of protecting them from the hazards of conventional excavation and waste transport. All work performed during testing adhered to the EG\&G Idaho Safety Manual and RAHCO's Safety Plan.

The RAHCO safety engineer conducted a safety walk-down of the test area daily. There were no safety discrepancies or concerns during the demonstration. However, before the tests, when RAHCO was preparing the pit and area, several concerns arose that were not raised as an issue until the demonstration. These concerns involved not wearing personal protective equipment (PPE) during preparation of the Portland cement drums. There were no injuries. During the demonstration, a safety meeting was held each day.

\subsection{Safety and Health for Phase I Testing}

Before the construction of the test bed, safety and health issues were addressed in the Demonstration Activity Hazard Analysis. Potential hazards were studied concerning the waste pile construction, actual test, and cleanup. The demonstration activities were deemed "Routinely Accepted by the Public."

During the steps of Phase I, the number of participants and observers was kept to a minimum. People who took an active role in the building and spiking of the simulated waste were required to first attend a safety meeting. At this meeting, it was explained that praseodymium (a rare earth tracer) would be spread in the simulated waste. The rare earth tracer is an irritant and can be irritating to eyes, respiratory system, and skin. Because the concentrations to be used in the demonstration were significantly greater than natural background, certain precautions were taken. Depending on potential level of exposure, PPE use and procedures were required as discussed in the test plan. ${ }^{4}$ The Material Safety Data Sheet (MSDS) for praseodymium was onsite at all times and explained to the participants. The praseodymium was stored in a locked room before spreading. All people in the area during the spiking process were required to wear respirators with cartridge filters, tight fitting (neck, cuffs, ankles) coveralls, hard hats, safety glasses with side shields, and rubber gloves. No contact lenses were allowed. Leather gloves were used when personnel worked with the machinery.

During the actual Phase I test, all participants and observers were given a safety orientation before being allowed into the test building. This consisted of pointing out that the viewing area was the balcony, safety glasses and hard hats were required, contact lenses were not recommended, and the building is a nonsmoking area. Only preapproved people were allowed on the test floor during the test. Their names and functions were maintained on a list, and they wore green identification badges during the test. Fire extinguishers and first aid kits were pointed out. Primary and secondary emergency phones were located and the reporting procedure was reviewed. Primary and secondary emergency escape routes were located. During the test, communications were verbal and by hand. Respirators, ear plugs (optional), safety glasses, hard hats, coveralls, rubber gloves, and an eye wash station were provided. BWID personnel wore optional steel-toed safety shoes. There was a written lock out/tag out procedure to follow when working on the excavator. It was also recommended that when leaving an area, personnel should wash their hands and faces and shower as soon as possible afterward. 
Electrical power for the demonstration was provided by RAHCO Building 1 power outlets. Fuses had to be repeatedly replaced on the excavator. The test director powered everything down while the fuses were replaced. No other electrical work was required.

The diesel exhaust from the excavator was ducted directly from the exhaust pipe to the outside atmosphere. The duct overheated and broke several times during testing and tests continued without venting the diesel. The test building is large and to limit exposure, the test durations were shortened as much as possible. The forklifts were kept off as much as possible to reduce the exhaust.

\subsection{Environment, Safety, and Health for Phase II Testing}

The Phase II safety and health issues were identical to Phase I with the exception that the viewing area was a roped off area and the engine exhaust was exhausted direct to the open air. There were flags to indicate the wind direction so exposure to potential dust clouds could be avoided. The first aid, safety equipment, and fire extinguisher station was located on the field site. All safety rules pertained to Phase I and Phase II.

Electrical power for the TTV control system was provided by a $24-\mathrm{V}$ dc generator belt-driven by the TTV diesel engine. Personnel did not access the TTV control system for any reason while the TTV engine was operating.

Electrical power for the remote control operations trailer was provided by a mobile generator. The power was turned on only for the time that power was needed during the testing. Access to the area around the generator set and the power lines were administratively controlled. Power lines were routed away from personnel pathways. Fuses had to be repeatedly replaced on the excavator. The test director powered everything down while the fuses were replaced.

The remote control operations trailer had an air conditioner to provide cooling. It was found that the air conditioner was positioned poorly relative to the door. When exiting the trailer, the air conditioner was on the left, at head level. Since the trailer door opened facing the TTV path, and the door hinges were opposite of the air conditioner, one tended to turn directly into the AC unit when exiting the trailer. This was reviewed in safety review meeting, and the air conditioning unit was marked with safety tape. No safety issues occurred as a result of this condition.

During measurement time-outs during the demonstration, the test director ensured via radio communication that all operations had ceased, equipment was in standby mode, and the area was clear for measurements. The test director was the only person authorized to give the "all clear" for measurement time-outs.

The TTV was equipped with several emergency stop functions. An emergency stop switch was located on the vehicle and on the remote control station. In addition, a supervisor's emergency stop belt module was used by the test director or designee. Additional details on the function of the emergency stop safety features is included in Section 7.2.4.3 H. 


\section{ENVIRONMENTAL OVERVIEW}

Many steps were taken to protect environment and minimize waste during the demonstration. In the simulated waste pit, recycled paper, used I-beam sections, and previously used large objects were used in the simulated waste pile. The waste was removed from test site and disposed at a landfill in accordance with local regulations. Following removal of the waste, the test site was restored to its original condition. During the nonactive portions of the demonstration, the waste was covered with plastic to prevent wind from spreading it from the test site. To simulate radioactive elements and monitor the spread of simulated airborne contaminants, praseodymium, a nonhazardous, nonradioactive rạe earth tracer was used.

In addition, the demonstration test site was a previously disturbed area and there were no previous contaminants on the test site. The test site was not located near streams, bodies of water, or residential areas.

National Environmental Policy Act (NEPA) documentation was approved as a Categorical Exclusion (CX) for the tests. The sources of impacts included air emissions, solid waste, water use/diversion, excess noise levels, chemical storage/use, soil surface disturbance, and petroleum storage. NEPA documentation was revised as the project changed (e.g., when the location of the demonstration was changed from the INEL to Spokane, Washington $\left.{ }^{b, c, d}\right)$.

b. J. F. Graham, "RWMC Cold Test Pit Waste Conveyance and IEE Demonstration (RWMC-93-018)," EG\&G Idaho, Inc. Interoffice Correspondence to B. M. Gardner, JFG-04-93, November 4, 1993.

c. J. F. Graham, "RWMC Cold Test Pit Waste Conveyance and IEE Demonstration (RWMC-93-018, Rev. 1)," EG\&G Idaho, Inc. Interoffice Correspondence to B. M. Gardner, JFG-02-94, January 11, 1994.

d. J. F. Graham, "Remote Conveyance and IEE/Dust Free Dumping System Demonstrations (RWMC-93-018, Rev. 2)," EG\&G Idaho, Inc. Interoffice Correspondence to B. M. Gardner, JFG-09-94, May 19, 1994. 


\section{TEST RESULTS}

Although the remote excavator was not part of the original test plan, ${ }^{4}$ it was a value-added item to the demonstration. Because of the opportunity to use the remote excavator, the opportunity to evaluate some aspects of its performance was taken. This information is included in Section 7.2 and throughout Section 7 .

\subsection{Phase I Demonstration Test Results}

This section presents results from the remote conveyance and IEE demonstrations for Phase I. This section is divided into five test objectives. The five objectives include (1) dust measurements and tracer measurements (Section 7.1.1), (2) operational evaluation, (3) effectiveness on various waste forms, and (4) overall Phase I demonstration success. The summary of the results, discussion, and conclusions are presented for each objective. Where appropriate, examples of data and analysis are included, with the remainder of the data and analysis included in the appendices. The Chemistry Laboratory ICP-MS report on the dust and rare earth tracer analysis is in Appendix D.

\subsubsection{Contamination Control Effectiveness}

During the Phase I testing, a total of five retrieval/dumping tests were performed to establish the magnitude of dust spread and the amount of tracer spread above background. Dust and tracer spread during these tests is analyzed for comparison of dust and plutonium spread during actual retrieval of TRU pits and trenches; therefore, evaluation of these parameters should give at least a qualitative indication of efficiency for contamination control during these operations. In these tests, the REMEX with IEE was used to dig in a specially prepared pit containing simulated waste material intermingled with dry INEL soil. Interlaced with the soil/waste matrix was the tracer praseodymium oxide used as a surrogate for plutonium/americium oxide, which occurs in actual transuranic waste pits. The soil used in this pit was extremely "dry" unconsolidated soil (moisture content averaged $8.23 \%$ by mass), considered a worst case for aerosolization of the finer soil particles and airborne contamination spread. The tracer was doped in the soil waste matrix at about 5 times background or $36 \mu \mathrm{g}$ of tracer per gram of soil (36 parts per million). This doping level is approximately 13 times the expected plutonium content of the INEL TRU pits and trenches (assumed 1,000 kg of plutonium/americium oxide in 8 million cubic feet of soil/waste matrix) and therefore should represent a conservative case for tracer spread evaluation during the digging/dumping operation associated with the Phase I testing.

7.1.1.1 Data and Data Reduction. Figure 15 shows a sample graph of the dust measured for the test backgrounds and test samples for one of the five test runs. The raw data, calculation of the mean and standard deviation, and the t-test analysis for this same test are presented in Table 2. The analytical technique used along with the complete analysis and data sets for each test run can be found in Appendix F.

Figure 16 shows a sample graph of the rare earth tracer measured for the test backgrounds and test samples for one of the five test runs. The raw data, calculation of the mean and standard deviation, and the $t$-test analysis for this same test are presented in Table 3 . The analytical technique used along with the complete analysis and data sets for each test run can be found in Appendix F. 


\section{Dust Measurement - Test \#5}

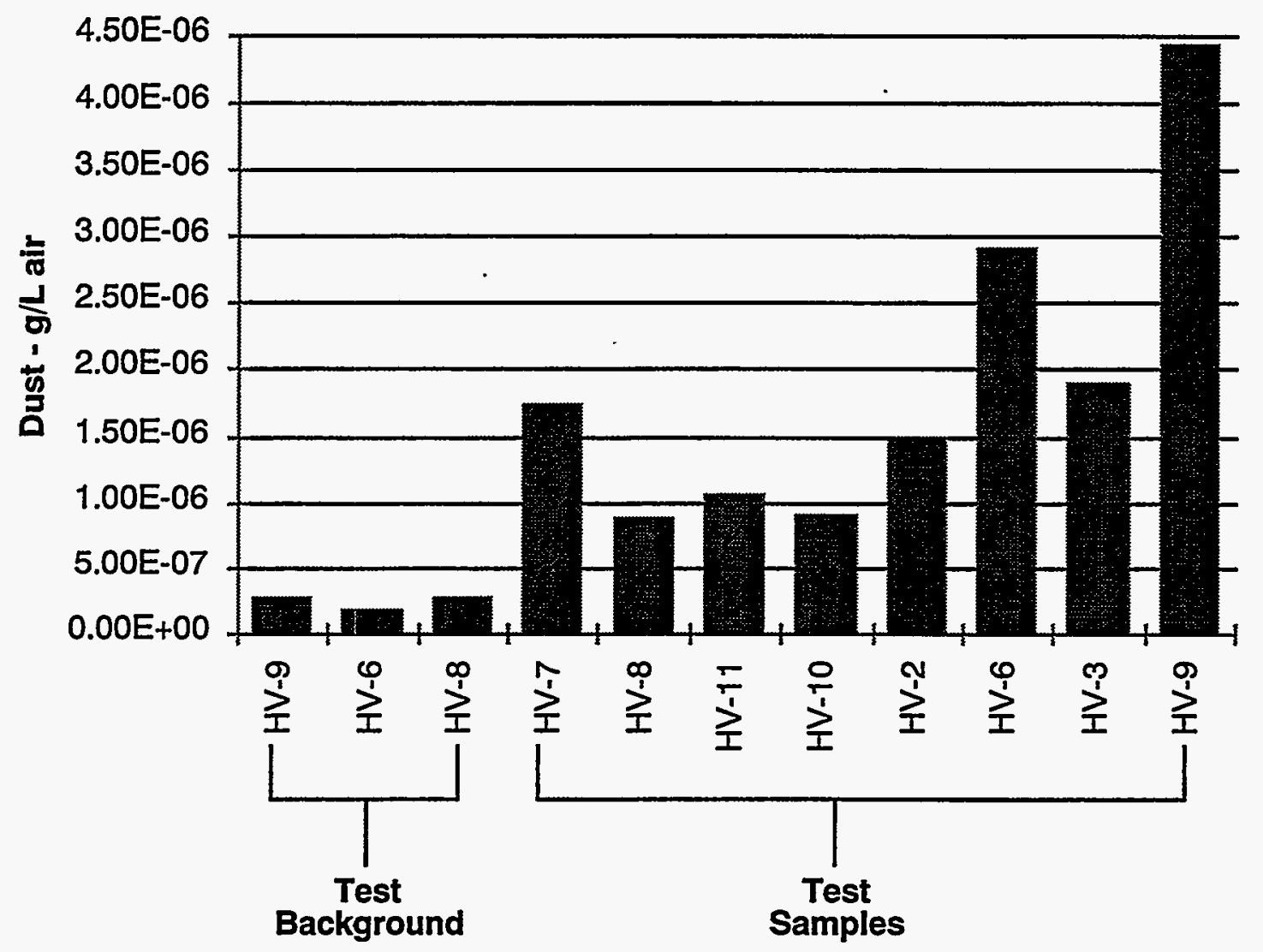

Air Sampler

Figure 15. The dust measurement graph for test 5 shows the test sample levels were considerably less than target of $1.1 \times 10^{-4} \mathrm{~g} / \mathrm{L}$-air above the detected background levels. 
Table 2. Data and analysis for the dust measurement for test 5 .

\begin{tabular}{|c|c|c|c|}
\hline Description & & Monitor ${ }^{\mathrm{a}}$ & $\begin{array}{c}\text { Dust loading } \\
\text { (g/L-air) }\end{array}$ \\
\hline \multirow{3}{*}{ Test background } & & HV -9 & 2.63E-07 \\
\hline & & HV-6 & $1.87 \mathrm{E}-07$ \\
\hline & & $\mathrm{HV}-8$ & 2.83E-07 \\
\hline \multirow[t]{9}{*}{ Test samples } & & $\mathrm{HV}-7$ & $1.75 \mathrm{E}-06$ \\
\hline & & $\mathrm{HV}-8$ & $8.85 \mathrm{E}-07$ \\
\hline & & HV-11 & $1.06 \mathrm{E}-06$ \\
\hline & & $\mathrm{HV}-10$ & 9.15E-07 \\
\hline & & HV-2 & $1.49 \mathrm{E}-06$ \\
\hline & & $\mathrm{HV}-6$ & 2.92E-06 \\
\hline & & $\mathrm{HV}-3$ & $1.89 \mathrm{E}-06$ \\
\hline & & HV -9 & $4.42 \mathrm{E}-06$ \\
\hline & \multicolumn{2}{|c|}{ Basis: test background } & \\
\hline Test mean & $=$ & $1.92 \mathrm{E}-06$ & \\
\hline Test std. dev. & $=$ & $1.21 E-06$ & \\
\hline nd & $=$ & 8 & \\
\hline$n u=(n d+n b-2)$ & $=$ & 9 & \\
\hline Critical 1-sided $\mathrm{t}(.95, \mathrm{nu})$ & $=$ & 1.83 & \\
\hline Pooled std. dev. & $=$ & 1.07E-06 & \\
\hline Critical value $t$ statistic & $=$ & -252.18 & \\
\hline Calculated P-value & $=$ & N/A & \\
\hline Background mean & $=$ & $2.44 \mathrm{E}-07$ & \\
\hline Background std. dev. & $=$ & $5.06 \mathrm{E}-08$ & \\
\hline nb & $=$ & 3 & \\
\hline (Test mean/background mean) & $=$ & 7.84 & \\
\hline
\end{tabular}


Rare Earth Tracer Quantities - Test \#5

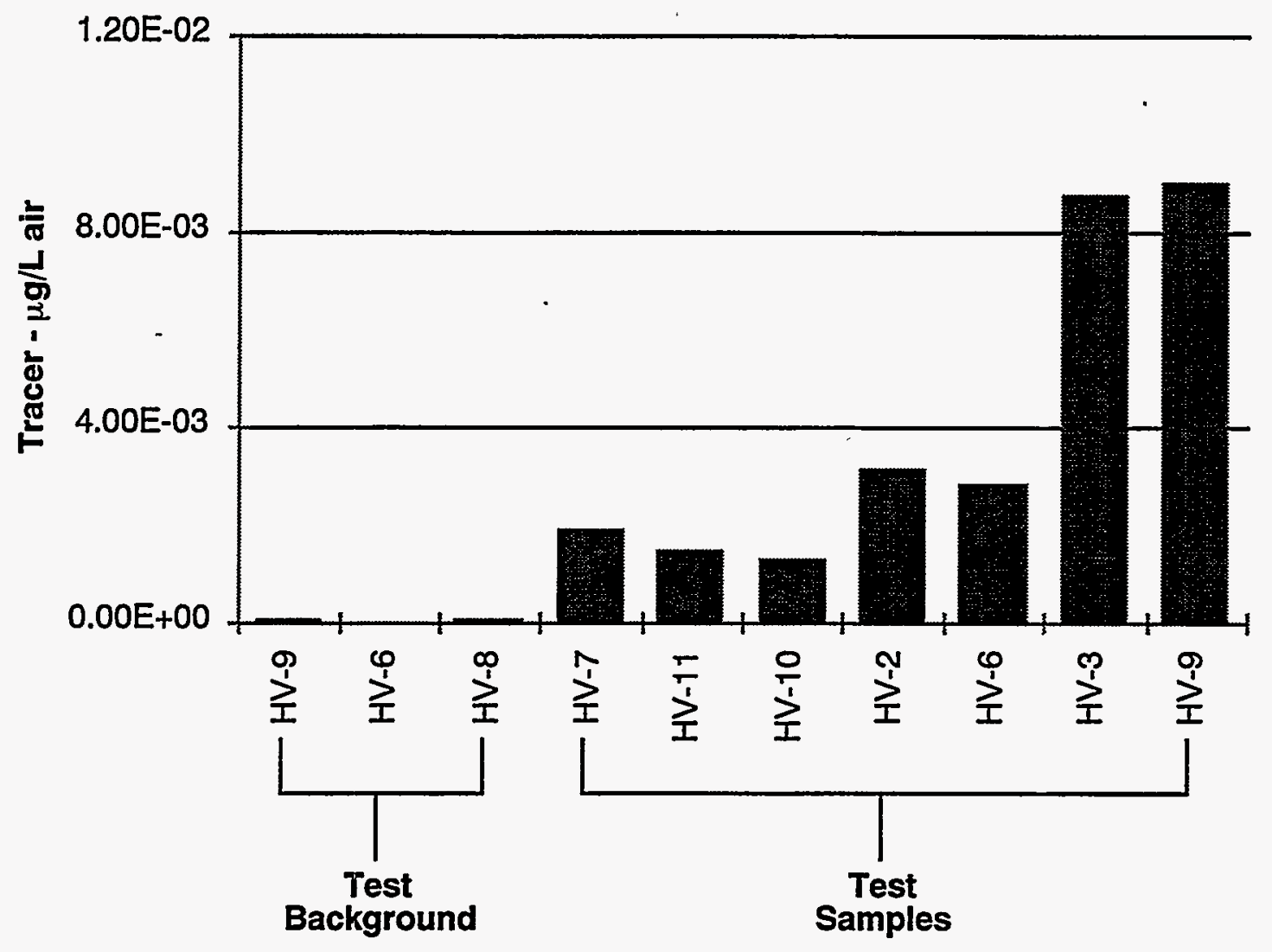

Air Sampler

Figure 16. The rare earth tracer quantities graph for test 5 shows the praseodymium test sample levels and the background levels detected. 
Table 3. Data and analysis for the rare earth tracer quantities test 5.

\begin{tabular}{|c|c|c|c|}
\hline Description & & Monitor $^{\mathrm{a}}$ & $\begin{array}{c}\text { Tracer loading } \\
(\mu \mathrm{g} / \mathrm{L} \text {-air })\end{array}$ \\
\hline \multirow[t]{3}{*}{ Test background } & & HV-9 & 3.60E-05 \\
\hline & & $H V-6$ & $1.70 \mathrm{E}-05$ \\
\hline & & $\mathrm{HV}-8$ & 3.01E-05 \\
\hline \multirow[t]{8}{*}{ Test samples } & & HV -7 & 1.93E-03 \\
\hline & & HV-11 & $1.48 \mathrm{E}-03$ \\
\hline & & $\mathrm{HV}-10$ & 1.32E-03 \\
\hline & & $\mathrm{HV}-2$ & 3.19E-03 \\
\hline & & $\mathrm{HV}-6$ & $2.84 \mathrm{E}-03$ \\
\hline & & $\mathrm{HV}-3$ & 8.78E-03 \\
\hline & & $\mathrm{HV}-9$ & 9.01E-03 \\
\hline & \multicolumn{2}{|c|}{ Basis: test background } & \\
\hline Test mean & $=$ & $4.08 \mathrm{E}-03$ & \\
\hline Test std. dev. & $=$ & $3.36 \mathrm{E}-03$ & \\
\hline nd & $=$ & 7 & \\
\hline Degrees of freedom & $=$ & 6 & \\
\hline Critical 1-sided $\mathrm{t}(.95, \mathrm{nu})$ & $=$ & 1.94 & \\
\hline Pooled std. dev. & $=$ & N/A & \\
\hline Critical value $\mathbf{t}$ statistic & $=$ & 3.19 & \\
\hline Calculated P-value & $=$ & N/A & \\
\hline Background mean & $=$ & 2.77E-05 & \\
\hline Background std. dev. & $=$ & $9.72 \mathrm{E}-06$ & \\
\hline $\mathrm{nb}$ & $=$ & 3 & \\
\hline (Test mean/background mean) & $=$ & 147.25 & \\
\hline
\end{tabular}

a. HV-\# = HI-VOL air sampler. For a diagram of HI-VOL placement, see Figure 19.

7.1.1.2 Data Analysis. The data quality objective for dust measurements was to determine whether dust collected exceeded the background by $1.1 \times 10^{-4} \mathrm{~g} / \mathrm{L}$-air. The $1.1 \times 10^{-4} \mathrm{~g} / \mathrm{L}$-air was based on a $98 \%$ dust removal for aerosolized soil in glove box experiments (see Appendix A). In these experiments, sieved soil (50 mesh) was aerosolized with air in the glove box environment and collected on filters. The $98 \%$ dust removal was chosen as a comparative value based on calculations of assumed source term in the SDA TRU pits and trenches $(450 \mathrm{nCi} / \mathrm{g})$ and past experience at the INEL with allowing personnel wearing bubble suits to enter the areas $(10 \mathrm{nCi} / \mathrm{g}$ or $2 \%$ of the source term).

The data quality objective for the rare earth tracer was to determine with a $95 \%$ confidence level if rare-earth tracer (praseodymium) was detected above background levels. 
To assess whether the dust and rare earth tracer detected on the air sample filters exceeded the background level of dust and tracer in the air (by whatever amount was desired) a statistical t-test was employed. This procedure tests the hypothesis of equality of the mean dust or tracer detected during operations to the background dust and tracer or target goal. The complete analytical procedure, data, and results are presented in Appendix F.

\subsubsection{Results.}

7.1.1.3.1 Dust-Since the t-statistic calculated in all cases is less than the critical 1-sided $t$ test (see Table 4), then the null hypothesis (Mean Dust Detected $\leq$ Background Dust $+1.1 \times 10^{-4} \mathrm{~g} / \mathrm{L}$-air) is not rejected. Thus, the average amount of dust detected on the air sample filters was not greater than $1.1 \times 10^{-4} \mathrm{~g} / \mathrm{L}$ of air above background levels for any of the tests in the demonstration. This conclusion is intuitively obvious since the observed dust loadings were two orders of magnitude less than the target . goal of $1.1 \times 10^{-4} \mathrm{~g} / \mathrm{L}$-air.

The dust measurement for test 1 experienced the highest average amount of dust detected at $5.54 \times$ $10^{-6} \mathrm{~g} / \mathrm{L}$-air compared with the lowest, test 5 at $1.92 \times 10^{-6} \mathrm{~g} / \mathrm{L}$-air. The elevated level for test 1 is probably due to operator inexperience (see Section 7.1.2). See Figure 17 for a comparison of test and background averages.

Examination of the detailed dust spread data for each of the samplers (see Appendix F) shows mixed results. For tests 1,2 , and 5, samplers 7 and/or 9, adjacent to the digging operation were higher than samplers 3,5,6,8,10, and 11 (adjacent to the dumping operation) as expected; however for tests 3 and 4, the opposite trend occurred (compare Figures F-1 through F-5 in Appendix F). The explanation for this phenomena is that the local interior building wind currents carried the small-sized particles (less than $20 \mu$ ) generally from the digging area to the dumping region. Additionally, the dumping operation is really a "deferred" dumping operation and only involves placing the filled ITM on the carrier. This operation is inherently "dust free" except for surficial soils attached to the outer bucket. It was obvious from visual observations and agreed upon by all those witnessing the demonstration that virtually all dust generated and collected on the air monitors came from the digging operations. Therefore, it is concluded that the source of dust spread for the subject demonstration was almost entirely from the digging

Table 4. The statistical analysis strongly supports the null hypothesis that the mean dust detected was, for all tests, less than the test goal of $98 \%$ dust removal.

\begin{tabular}{ccccc}
\hline $\begin{array}{c}\text { Test } \\
\text { number }\end{array}$ & Calculated t statistic & $\begin{array}{c}\text { Critical 1-sided } \\
t(.95, \text { nu) }\end{array}$ & Null hypothesis & $\begin{array}{c}\text { Do not reject } \\
\text { or reject }\end{array}$ \\
\hline & & & & \\
1 & -45.408 & 1.89 & $H_{O}: u_{d} \leq u_{b}+1.1 \times 10^{-4}$ & Do not reject \\
2 & -209.22 & 1.83 & $H_{o}: u_{d} \leq u_{b}+1.1 \times 10^{-4}$ & Do not reject \\
3 & -267.68 & 1.83 & $H_{O}: u_{d} \leq u_{b}+1.1 \times 10^{-4}$ & Do not reject \\
4 & -199.49 & 1.83 & $H_{O}: u_{d} \leq u_{b}+1.1 \times 10^{-4}$ & Do not reject \\
5 & -252.18 & 1.83 & $H_{O}: u_{d} \leq u_{b}+1.1 \times 10^{-4}$ & Do not reject \\
\hline
\end{tabular}




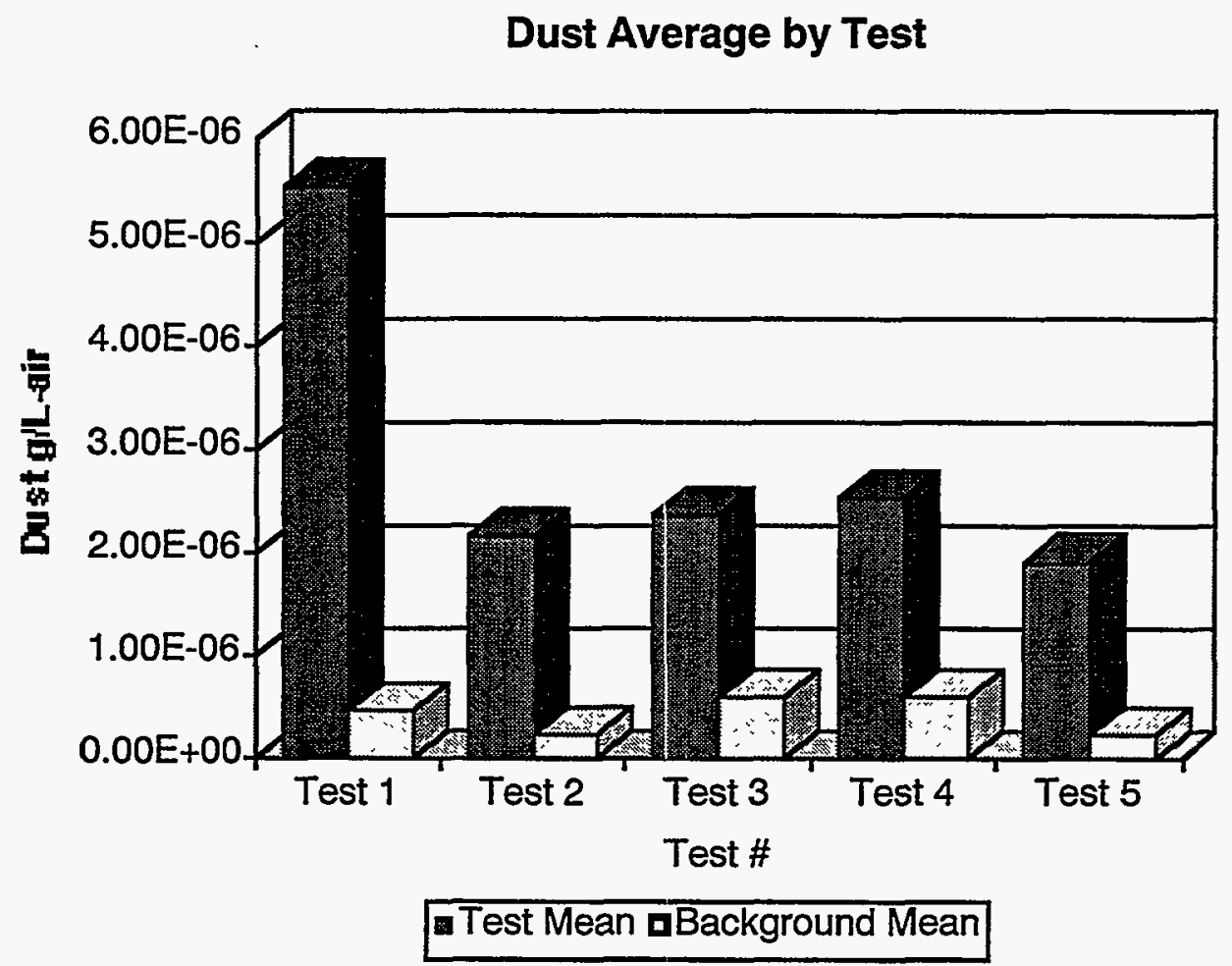

Figure 17. The test means for the five dust measurement tests clearly show that all of the dust samples taken are significantly (two orders of magnitude) below the target goal of $1.1 \times 10^{-4} \mathrm{~g} / \mathrm{L}$-air.

operation and variable small wind currents inside the building carried the dust to the various filters in a variable manner. This statement is supported by the data displayed in Figure 18, which shows the average dust collected for each air monitor across all the tests. HV-9 (the air monitor closest to the digging in the path of the movement of the dust) shows significantly higher dust loadings than the rest of the air monitors. HV-9 was closest to the dig pile and along the direction of the prevailing movement of the dust generated during digging (see Figure 19). This also supports why HV-7 has one of the lowest average dust loadings. Although it was also next to the pile, the dust cloud was always moving away from this air monitor and the waste wall partially shielded the monitor.

As previously discussed, much more dust was generated during the first test than any of the rest. The reason for this is that the operator of the excavator had little practice with the IEE before the start of the demonstration and also had no experience digging in a waste pile like the one built for this demonstration. After the operator gained some experience and was instructed by the test conductors on how to more effectively retrieve the waste, the amount of dust generated was significantly reduced for the remainder of the demonstration.

7.1.1.3.2 Rare Earth Tracer-The t-statistic calculated in all but one case (test 1 ) is less than the critical 1-sided t-test (see Table 5). The null hypothesis (Mean Praseodymium Detected = Background Praseodymium) is therefore rejected in favor of the alternative hypothesis (Mean 


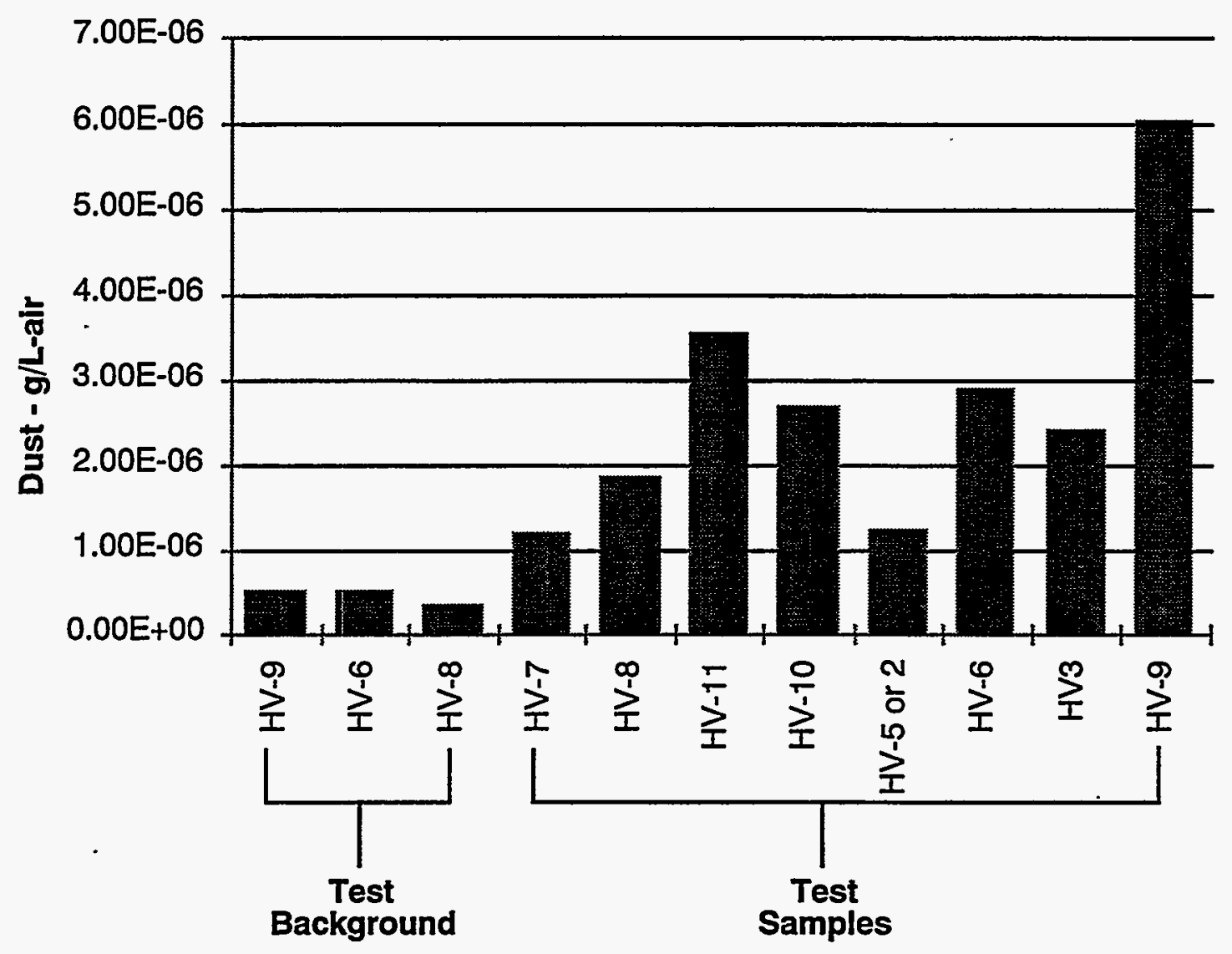

Monitor Number

Figure 18. The average dust collected for each air monitor during the demonstration clearly shows that HV-9, the monitor closest to the digging, had the highest average dust loading. This clearly supports the visual observations that virtually all dust generation resulted from digging. Dumping (or placement of the ITM) created virtually no dust. 


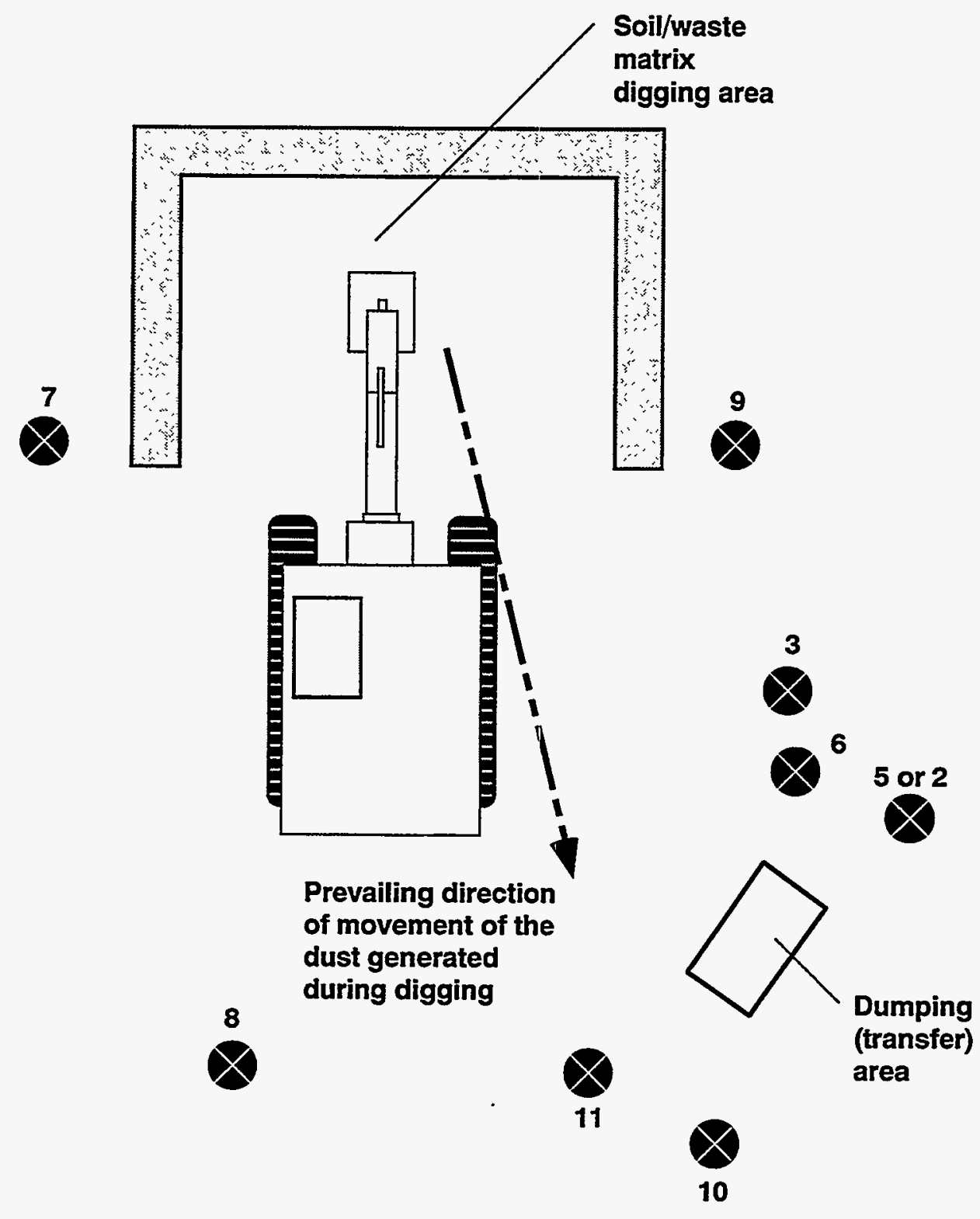

X940502

Figure 19. Placement of the air monitors for the Phase I demonstration was intended to isolate the dust generation caused by digging from that caused by dumping. Prevailing air currents in the building carried the dust from the digging zone out across the test arena toward the dumping zone. However, the air monitor data (especially from HV-9) along with visual data clearly show that the dumping operation was performed virtually dust free. 
Table 5. The statistical analysis supports the alternative hypothesis that the mean tracer detected was, for most tests, greater than the background levels.

\begin{tabular}{ccccc}
$\begin{array}{c}\text { Test } \\
\text { number }\end{array}$ & Calculated t statistic & $\begin{array}{c}\text { Critical 1-sided } \\
\mathrm{t}(.95, \mathrm{nu})\end{array}$ & Null hypothesis & Do not reject or reject \\
\hline & & & & \\
1 & 2.12 & 2.13 & $H_{o}: u_{d}=u_{b}$ & Do not reject \\
2 & 3.30 & 1.83 & $H_{o}: u_{d}=u_{b}$ & Reject \\
3 & 5.16 & 1.83 & $H_{o}: u_{d}=u_{b}$ & Reject \\
4 & 4.29 & 1.83 & $H_{o}: u_{d}=u_{b}$ & Reject \\
5 & 3.19 & 1.86 & $H_{O}: u_{d}=u_{b}$ & Reject \\
\hline
\end{tabular}

Praseodymium Detected $>$ Background Praseodymium). The average amount of Praseodymium detected on the air sample filters was greater than background levels for four of the five tests in the demonstration. Failure to determine that the tracer detected exceeded the background levels for the first test is a statistical problem caused by the failure to meet the data quality objective for this test. This was due to a power failure that caused several of the air monitors to prematurely shut down. Statistically speaking, the lack of enough data and the high variance of the data collected made it impossible to determine if the tracer detected was higher than background. Practically speaking, the data obviously show that the tracer levels detected were above background. This is indicative of a failure to meet a data quality objective (i.e., the quality of the data was not enough to make a statistically significant determination of the results).

The rare earth tracer measurement for test 1 was the highest average amount of tracer detected at $5.44 \times 10^{-3} \mu \mathrm{g} / \mathrm{L}$-air compared with the lowest, test 3 at $1.59 \times 10^{-3} \mu \mathrm{g} / \mathrm{L}$-air. See Figure 20 for a comparison of the test averages and background averages.

For all sampler positions, analysis of the filters for tracer concentration showed that tracer was spread to all sampler positions during the combined digging/dumping operation. All filter positions showed tracer concentrations above background as shown in Figure 21. For all five tests, the ratio of the test mean data to the background mean data is greater than 1 . The range of rare earth tracer concentrations on the filters was between 9.18 and 224.19 times the background levels. Again, as with the dust data, the average tracer collected for each air monitor during the demonstration (see Figure 21) clearly shows that HV-9, the monitor closest to the digging, had the highest average tracer loading. This clearly supports the visual observations that virtually all dust generation resulted from digging. Dumping (or placement of the ITM) created virtually no dust.

Note: Only simple statistical techniques were used to examine the relationships between each dust monitor and across tests. Assumptions about the variance across tests were made based on similar patterns of dust and tracer loading for each test and upon visual observations. More sophisticated statistical techniques could yield interesting data relationships; however, the capability of the author to perform these tests (minimal), and the availability and cost of statisticians to perform more thorough analysis, prohibited expansion of the analysis. A statistician was consulted and reviewed the results. There did not seem to be a great deal of value in performing a more in-depth statistical study of the results. This will be left to the reader. 


\section{Rare Earth Tracer Average by Test}

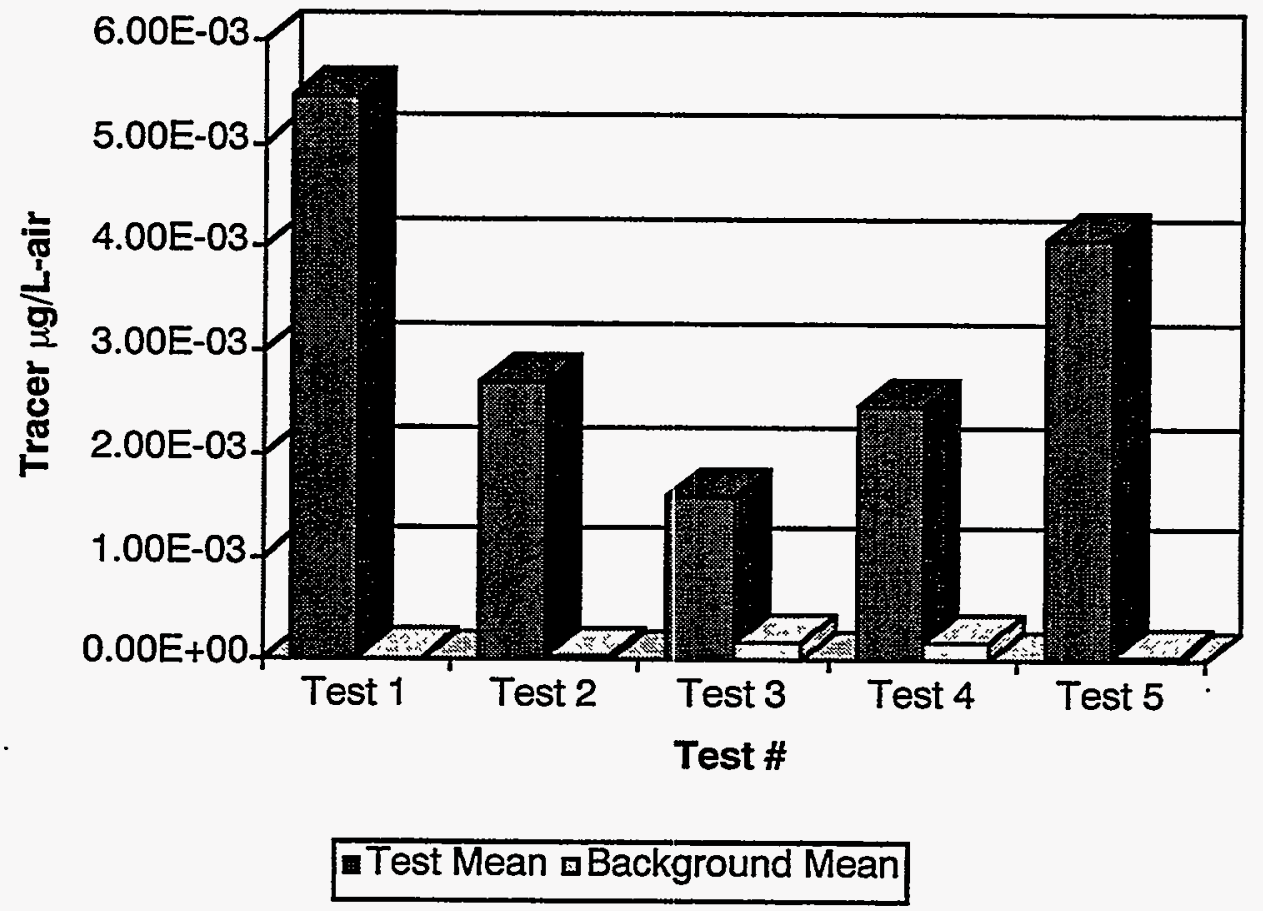

Figure 20. Test and background means for the five rare earth tests show that tracer was detected, confirming the movement of particles of praseodymium from the source. 


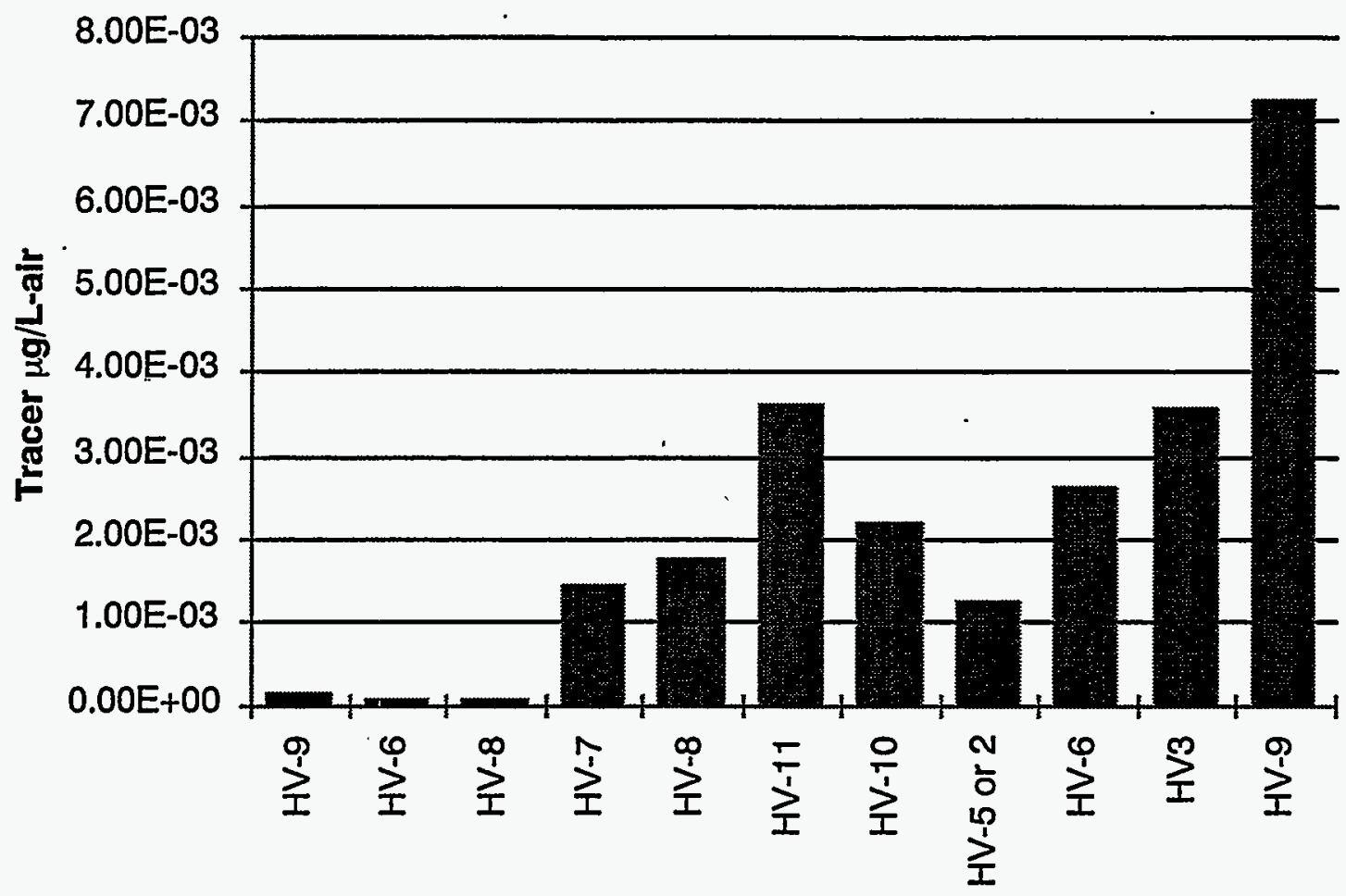

Monitor Number

Figure 21. The average tracer collected for each air monitor during the demonstration shows that tracer was detected at levels significantly above background. The significance of this is that it confirms the source of the dust and indicates potential contamination spread.

A more detailed examination of the data presented in Appendix F shows that virtually all filters in each of the five tests showed tracer collection above the background values. Again, as with the dust spread data, examination of the individual filters showed mixed results. It would be expected that the samplers located nearer to the inherently dusty digging operation (sampler 7 and 9) would show more tracer concentration than the samplers located nearer the transfer point for the ITM; however, the data shows for tests 1,2 , and 5, sampler number 9 had higher concentrations, but the samplers nearer the transfer point had higher concentrations for tests 3 and 4. There is considerable variation in the ratio of test mean to background mean from test to test. There is also considerable variation between average tracer collected for each monitor across the tests (see Figure 21). One speculation for this result is the variability of how tracer was placed in the simulated pit. From one test to another, the digging occurred in various regions of the pit involving potentially different waste materials. These different materials could have caused more difficulty in the digging operation - more or less mechanical shock - resulting in more or less dust spread and, therefore, tracer spread. In addition, the tracer was not spread homogeneously throughout the waste pile, but was applied in layers with more concentrated on waste forms than on loose soil. The variation in measured tracer amounts might possibly be related to the specific area and waste forms being excavated. Again, as with the dust spread measurement, visual observations of the process showed that no visible dust (or tracer) was seen for the transfer of the ITM, however, for the digging operation, considerable dust was generated. Visual data supports that even though sampler numbers 7 and 9 were located near the digging operation, prevailing laminar air currents inside the building carried dust and tracer either away from the sampler (as is the case with HV-7) or into the sampler (as happened 
with HV-9). This explanation assumes that tracer and dust move together and in approximately the same manner. Analysis of the correlation between dust and tracer loadings on the filters (discussed in Section 7.1.1.4) supports this assumption.

7.1.1.4 Conclusion. Based on the original data quality objective, the overall process of using the IEE resulted in dust spread that was less than that considered for the success criteria, indicating a positive proof-of-concept. For the original data quality objective, the mean of all tests was a factor of 44 less than the success criteria. Examining the detailed data in Appendix F shows that virtually every sampler met the criteria of collecting less dust than $1.1 \times 10^{-4} \mathrm{~g} / \mathrm{L}$-air, supporting the idea that the criteria set forth in the test plan was successfully met and a positive proof of concept was shown.

On an overall basis the transfer of the ITM from the excavator to the transfer point was accomplished with no visible dust or tracer spread as expected. However, when combining the inherently dusty operation of digging with the dumping/transfer operation, there was considerable (although lower than the target goal) dust and tracer spread throughout the entire test area. The source of the dust generation was the digging operation alone, which caused tracer spread above background in all sampler locations. The data for dust spread measurements showed that virtually all samplers met the original criteria of being below $1.1 \times 10^{-4} \mathrm{~g} / \mathrm{L}$-air (by 2 orders of magnitude); however, when compared to sampler data taken in similar digging and dumping experiments in INEL full-scale baseline experiments with no contamination control applied, the IEE resulted in dust spread measurements on the same order of magnitude $\left(1 \times 10^{-6} \mathrm{~g} / \mathrm{L}\right)$. This is due to the inherently dusty digging operation regardless of end effector, the very dry nature of the subject test soil compared to the INEL full-scale baseline soil, and the fact that no contamination control was used at the digface. The data for the tracer spread showed that virtually all sampler positions were above background levels of the tracer. Again, the tracer spread is due to the inherently dusty digging operation regardless of end effector.

It is recommended that a positive form of dust control be applied at the digging site to reduce the overall dust and tracer spread during the digging operation. Controlling the dust generation at the source of digging combined with the IEE's inherently dust free dumping operation should result in substantially greater than $98 \%$ reduction in dust spread over cases without regard for contamination control. This 98\% dust control should result in an acceptable reduction in contaminant spread during TRU retrieval operations for areas similar to the INEL SDA pits and trenches (see Appendix A). However, when looking at "as low as reasonably achievable" (ALARA) criteria, these test results indicate that combining active dust control during the digging operations with this dust free dumping system, significantly more stringent criteria (such as $1.0 \times 10^{-6} \mathrm{~g} / \mathrm{L}$-air) should be applied. These criteria should be considered for use in the test and data quality objectives for BWID follow-on tests.

The purpose of performing rare earth tracer analysis has traditionally been to confirm the source of potential airborne contamination spread (dust). An interesting finding in this demonstration is the almost perfect correlation ( 0.98 with 1.0 being perfect) between dust and rare earth tracer spread (see Figure 22). Because of past difficulty in controlling dust sources, it is necessary to perform the rare earth tracer study. The implications of this are that if performing a demonstration inside a structure that sufficiently controls the environment, and only a single source of potential dust exists, then the rare earth tracer study need not be performed. This can lead to a tremendous cost savings because the ICP-MS analysis of the filters for rare earths is expensive. 


\section{Dust and Rare Earth Tracer Correlation}

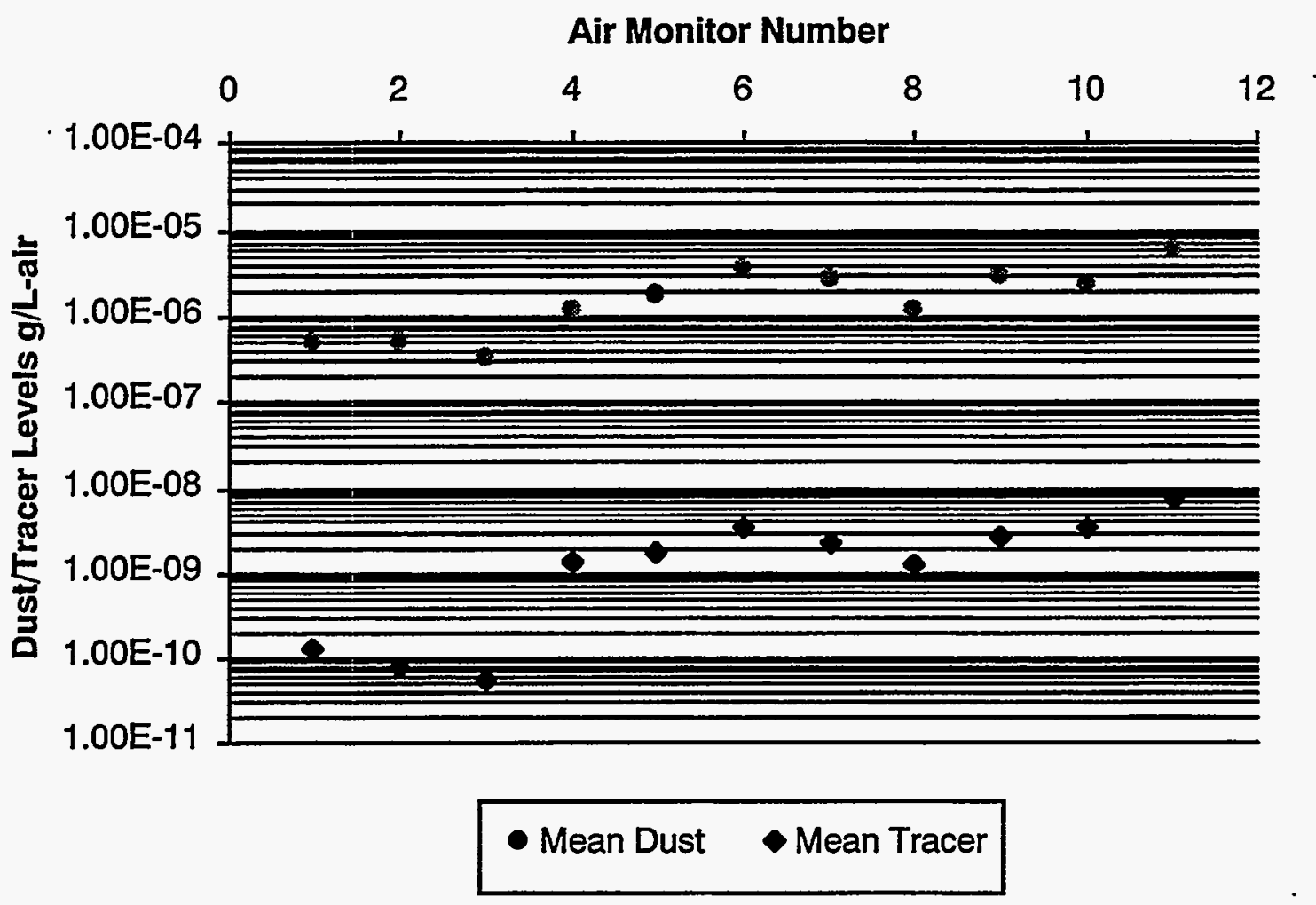

Figure 22. Dust and rare earth tracer data show an almost perfect correlation ( 0.98 with 1.0 being perfect) for the demonstration.

Correlations were calculated and plotted between the soil moisture content and average amount of dust and rare earth tracer measured on the filters for each test (see Appendix F). The correlation coefficient for soil moisture versus dust was -0.98 (significant for a levels of 0.05 and 0.01 ), and for soil moisture versus tracer was -0.66 (not significant) indicating on the surface that a strong correlation exists between soil moisture and dust generation. This should be correct and probably is; the more soil moisture, the less dust. This should also be true of tracer, but the data do not bear this out. This is probably due to the lack of a sufficient number of data points, variability across the data, and the nonhomogeneous application of the tracer to the waste pile.

\subsubsection{Operational Evaluation}

One of the data quality objectives for Phase I testing was to observe the operations of the IEE system and record information concerning: spillage, ability to handle waste forms, visual estimate of dust generation, reliability, ease of use, ease of maintenance, volume of waste removed per excavation cycle, and rate of removal. This section discusses data collection, reduction, analysis, results, and conclusions.

7.1.2.1 Data and Data Reduction. Phase I consisted of five tests. A summary of the test time, volume of waste removed, removal rate, and spillage is given in Table 6. Appendix G presents raw test data. 
Table 6. Summary of data for Phase I testing.

\begin{tabular}{ccccc}
\hline Test number & $\begin{array}{c}\text { Test time } \\
\text { (minutes) }\end{array}$ & $\begin{array}{c}\text { Volume removed } \\
\left(\mathrm{yd}^{3}\right)\end{array}$ & $\begin{array}{c}\text { Removal rate }^{\mathrm{b}} \\
\left(\mathrm{yd}^{3} / \mathrm{h}\right)\end{array}$ & $\begin{array}{c}\text { Spillagec }^{\mathrm{c}} \\
\left(\mathrm{yd}^{3}\right)\end{array}$ \\
\hline 1 & 57 & 4.04 & 4.25 & None \\
2 & 56 & 8.27 & 8.86 & 0.00060 \\
3 & 47 & 9.29 & 10.30 & 0.00015 \\
4 & 27 & 10.38 & 23.10 & 0.00180 \\
5 & 47 & 9.01 & 11.50 & 0.00030 \\
& & & \\
a. Total volume removed $=41.0 \mathrm{yd}^{3}$ & & & \\
b. Total volume spilled $=0.0042 \mathrm{yd}^{3}$ & & & \\
c. Average removal rate $=11.6 \mathrm{yd}^{3} / \mathrm{h}$ &
\end{tabular}

The spillage and volume of waste removed per excavation cycle was estimated and recorded in the logbooks for each cycle. A cycle is the time it took from picking up the empty ITM from the transfer station until releasing the full ITM to the transfer station. Spillage volume was estimated. visually. The volume of waste was estimated by using volume markers inside the ITMs. These markers were at $1 / 2$, $3 / 4$, and $7 / 8$ of a full ITM. A full ITM has a volume of $1.4 \mathrm{yd}^{3}$. The measurement was recorded by visually looking at the nearest marker and estimating the volume that was above or below the marker. The estimated accuracy of the volume measurements is $\pm 1 / 8$ of an ITM or $0.175 \mathrm{yd}^{3}$.

The rate of removal was calculated by dividing the volume retrieved by the time it took to remove that volume. The volume and times were recorded for each cycle. Time was recorded to the nearest second using a digital watch. The accuracy of the time measurement is estimated to be \pm 1 second. The average removal rate for each test was obtained by adding the total volume retrieved and dividing that volume by the total time it took to retrieve that waste.

Other information was qualitatively assessed. This information included the ability of the IEE to handle waste forms, a visual estimate of dust generation at the digface and transfer location, reliability of the IEE, ease of use of the IEE, and ease of maintenance of the IEE. Qualitative information regarding how well the IEE handled the types of waste forms was recorded in the logbooks. A visual estimate of dust generation was noted and was qualitatively described in the logbooks. Data for evaluating the reliability and maintainability of the systems was taken by documenting failures and maintenance activities.

7.1.2.2 Data Analysis. The data in Table 6 represents the averages from each test. The complete data set is given in Appendix G. The data were recorded for each cycle for each test. Each test represents the average of all of the cycles for that test. No data were judged invalid or unreasonable. 
Again, the rate of removal was calculated by dividing the volume retrieved by the time it took to retrieve that volume.

7.1.2.3 Results. There were two key factors that played a role in the success of Phase I testing: operator proficiency and design of the IEE. The results of the Phase I tests for evaluating the operations are given below.

\section{A. Spillage}

The test data in Appendix $G$ show the various waste forms removed and the spillage that was generated during each cycle. Out of $41 \mathrm{yd}^{3}$ of waste removed, there was only an estimated $0.0042 \mathrm{yd}^{3}$ of spillage. All spillage occurred within 1 to $2 \mathrm{ft}$ of the area and probably would have all been contained in the WTC had it been used. The WTC was not used for most of the Phase I testing to allow for easier ITM removal.

\section{B. Removal Rates}

Table 6 shows the average removal rates for each test. The test 1 removal rate was substantially lower than the others. After test 1 , modifications were made to the IEE (as discussed under item C) that allowed the IEE to operate more efficiently. The removal rates from the other four tests approached or exceeded the goal of $80 \mathrm{yd}^{3} /$ day. These removal rates were for the cycle time of getting the ITM, filling the ITM, and releasing the ITM at the transfer station and did not include any conveyance time. Another interesting result was the removal rate from test 4 of over $23 \mathrm{yd}^{3} / \mathrm{h}$. The crew performed this test just before closing and was highly motivated to perform the test faster.

\section{Ability of the IEE to Handle Waste Forms}

The design of the IEE proved to be effective in excavating all of the various waste forms. There were a few design modifications done to the IEE to improve its efficiency. The first modification involved reducing the overflow opening on the IEE. Initially, the waste would fall through the opening so that only $25 \%$ of the waste being excavated was captured in the ITM. This spillage created high dust levels at the digface. The second modification involved replacing the end effector ripper teeth with a grader bar and also adding a plate to the tip of the thumb. Figures 23 and 24 show the IEE before and after modification. The teeth and the thumb punctured drums and also caused debris to hang up on the IEE. Once modifications were completed, the IEE efficiently handled all waste forms. Figure 25 shows the IEE picking up drums and a beam. The test data show that the rate of removal more than doubled after these modifications were completed (after test 1), as shown in Table 6. Additional details on the ability of the IEE to handle the specific waste forms encountered is in Section 7.1.3.

\section{Dust Generation}

The operator began the demonstration with only 2 hours of practice with the IEE. This practice consisted of mating the IEE to the ITMs, latching and unlatching the ITMs, and general 


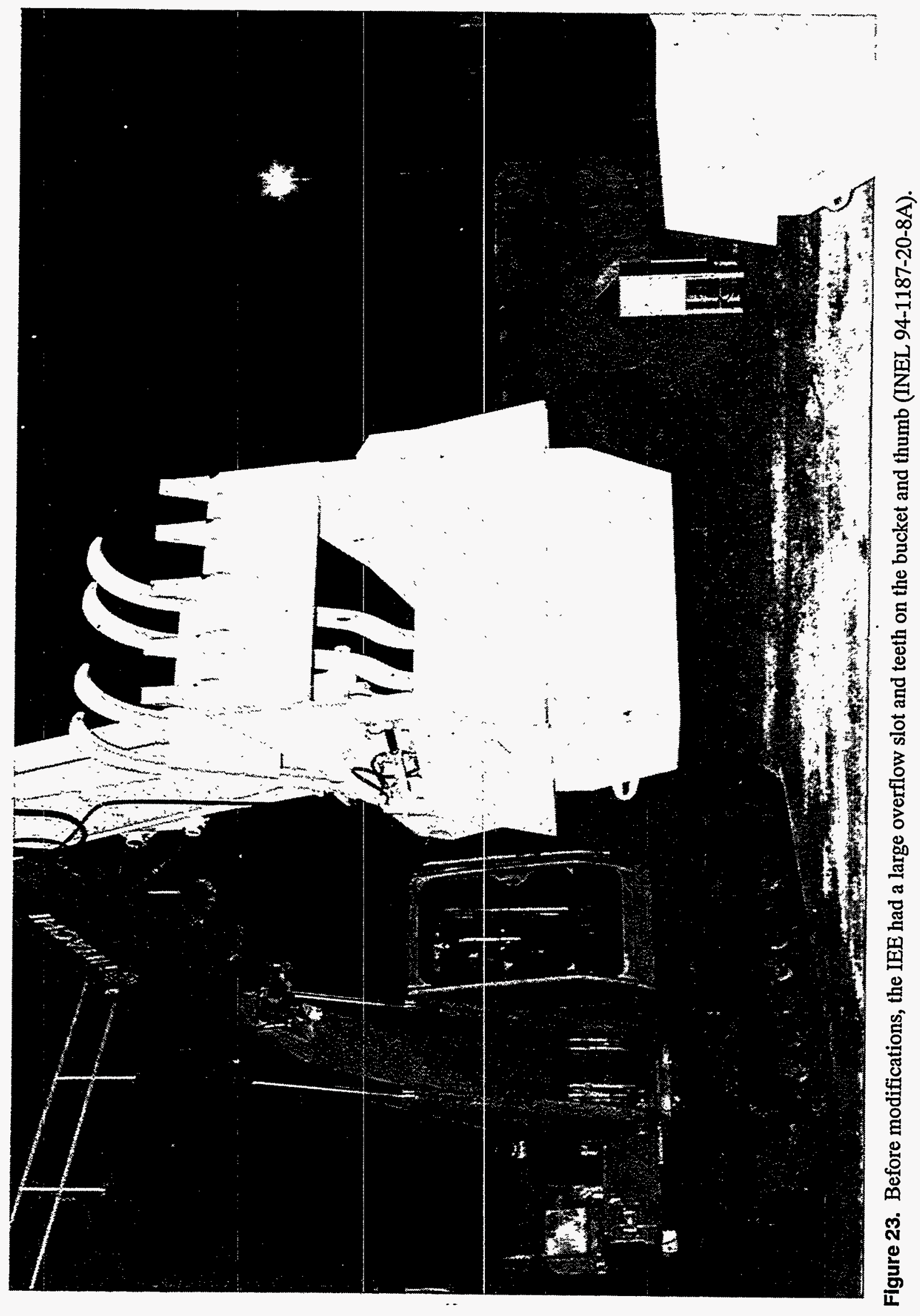



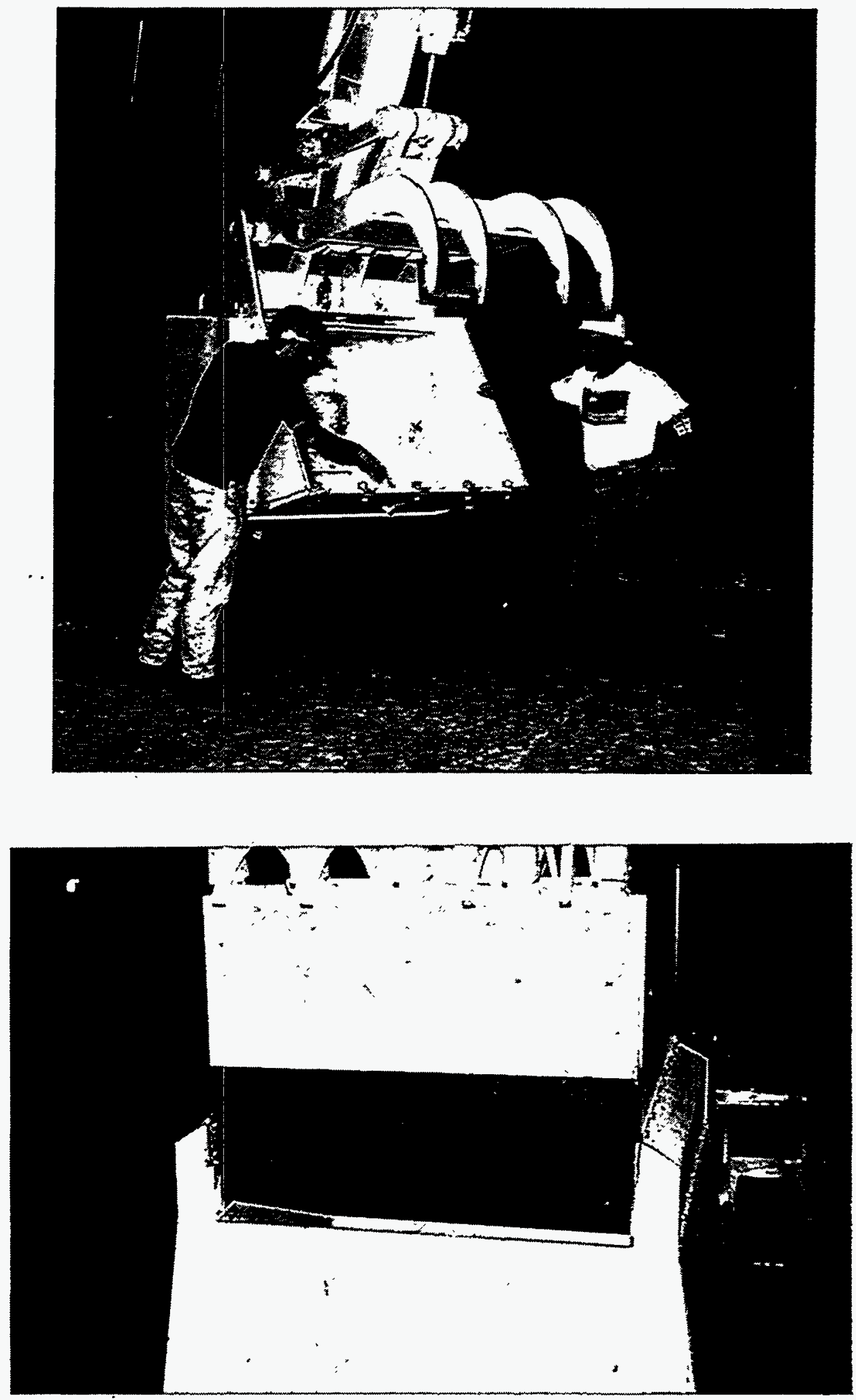

Figure 24. The IEE was more effective at handling the waste forms and filling the ITM after minor modifications were made (INEL 94-1187-18-12 and 94-1187-32-1). 


$$
\text { -....... - }
$$




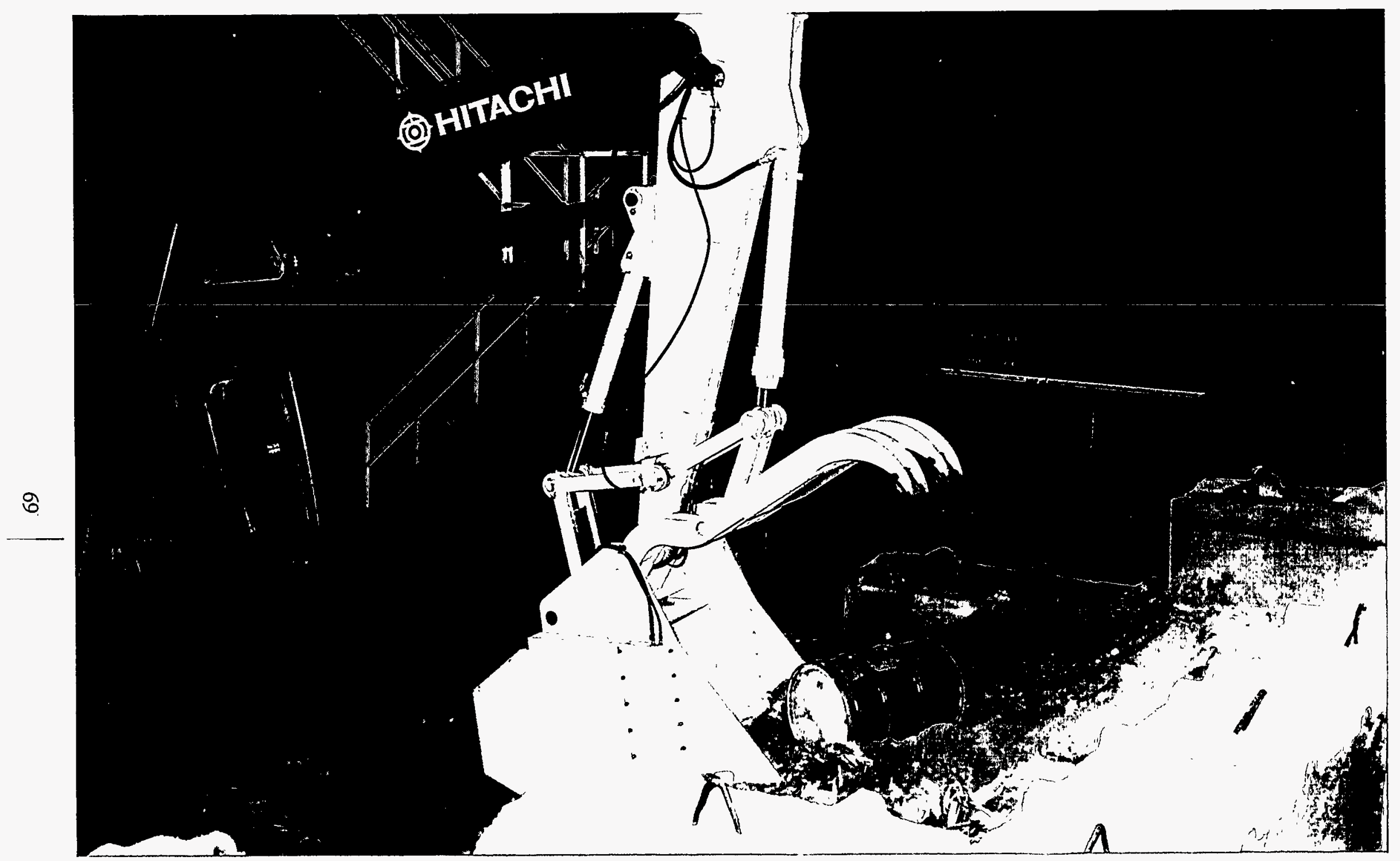

Figure 25. The IEE was flexible in its ability to pick up drums in two ways: either by depositing the drums into the attached ITM (as shown) or by using the bucket and thumb to pick up the drum and then deposit it into a detached ITM. The drums were filled with dry Portland cement (INEL 94-1187-29-4). 

operation of the excavator. The operator had no actual excavating practice with this equipment before testing. Consequently, during test 1 the end effector breached several drums which created large amounts of visible dust at the digface. Although the operator had difficulty at the digface during test 1 , the transfer operation was accomplished with little spillage. The ITM placement into the WTC was successful each cycle, and there was virtually no visible dust generated during the transfer cycles. Figure 4 shows an ITM being deposited into the WTC. Any dust generated during the transfer operation was completely contained by the WTC. See Table 6 for a summary of spillage from each test. Note that the WTC was not used after test 1 because the manual WTC dumping operation was delaying the excavating progress. Following test 1 , the operator's ability improved rapidly. The operator became more confident and moved the excavator in a fluid motion. It was found that with the operator in the excavator cab, the ITM bucket blocked the operator's view of the digface. This impaired the ability to use the thumb to pick up specific objects and the ability to see how much and what type of waste was entering the ITM. To compensate for this, the operator found that it was effective to detach the ITM and use the empty IEE to "arrange" the digface, or to pick up objects with the thumb and set them into the separated ITM. The process of moving items around on the digface to prepare for an ITM attached scoop generated visible dust at the digface.

Overall, the operator adapted quickly to the new system. With experience, the operations became more fluid and the dust generated at the digface was reduced. More importantly, the dust spread at the transfer stage was minimal (see test results in Appendix F). All spillage occurred within 1 to $2 \mathrm{ft}$ of the area and probably would have been contained in the WTC had it been used.

The system was operated in a front shovel configuration rather than a backhoe configuration. The front shovel configuration provides an advantage in dust control at the digface because the disturbed waste tends to fall into the bucket. The disadvantages in terms of reduced visibility and the resulting increased dust perhaps outweigh the initially expected advantages. Remote operations with a camera system that provided several different views of the digface would alleviate this problem.

E. Reliability of the IEE

Overall reliability of the IEE was high. Once the initial design modifications were completed and the maintenance issues resolved, the IEE operated continuously for the remainder of Phase I testing as well as Phase II testing. The rugged, stout design of the IEE held up well to the rough task of excavating the various waste forms and to the bumps and knocks of the retrieval process.

F. Ease of IEE Use

Mating of the IEE to the ITM was successful and was easily repeated every cycle. The flared skirting on the IEE and ITM proved to be an effective design and assisted with the attachment. Figure 26 shows the IEE mating to the ITM. Although the latching and unlatching mechanisms work well, the cycle time of 16 seconds per cycle could be reduced in future iterations. 


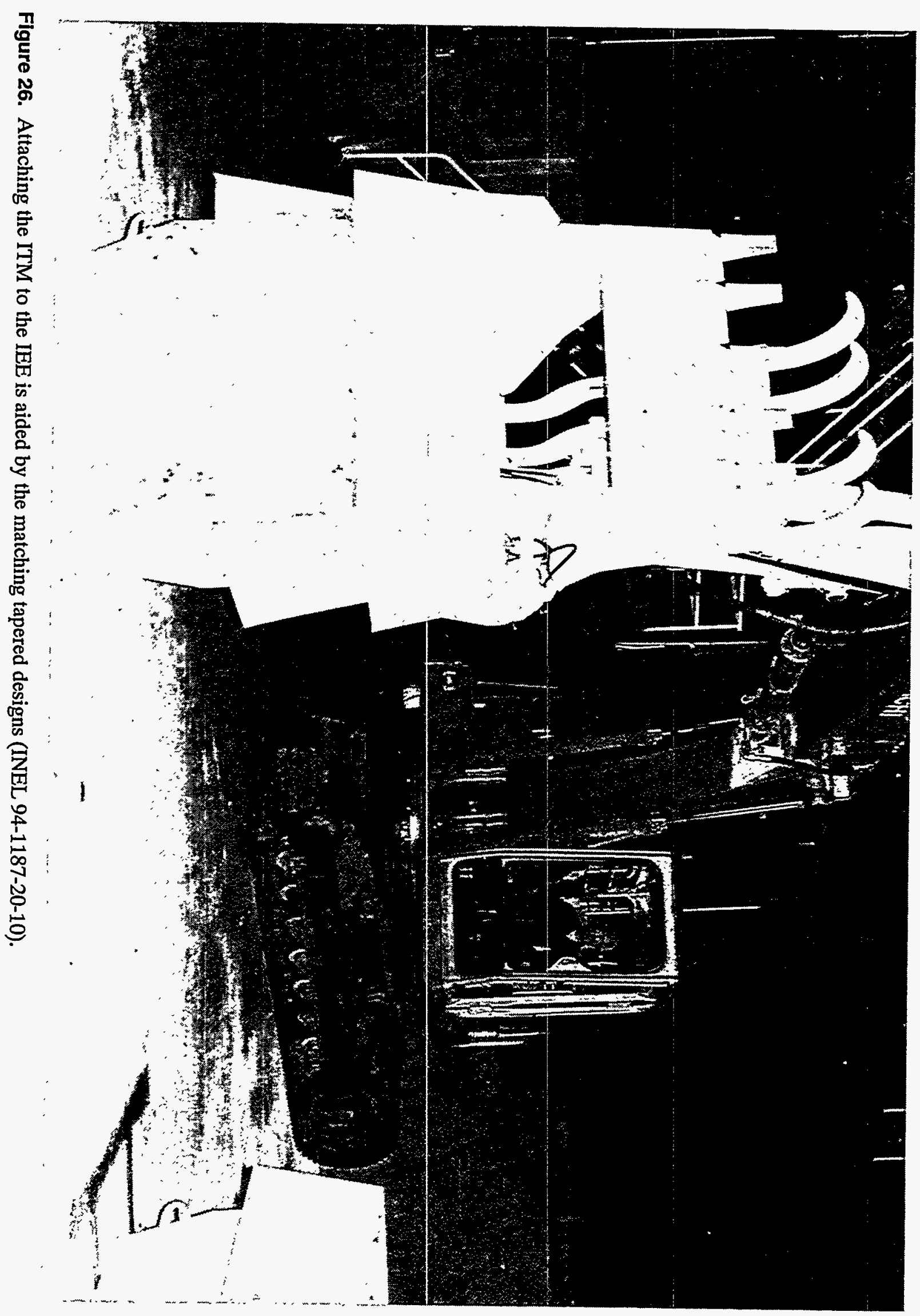


The IEE is easy to use as shown by the operator's ability to become proficient in a short period of time. The operator's visibility of the digface was limited by the ITM. By removing and not using the IEE as part of the end effector, the visual impairment was eliminated and in fact visibility was better than could be expected from a stand and shovel. The operator was able to operate in this mode and to move some objects into the ITM using only the thumb and end effector. For remote operations, this is not an issue because the remote cameras provide views other than those of the operator in the cab. The operator's depth perception and other human factors aspects of the IEE and vision system are discussed in greater detail in Section 7.4.

\section{G. Ease of IEE Maintenance}

The modifications done to the IEE to improve its efficiency created unscheduled maintenance outages that were completed in 2 hours. Another unscheduled maintenance item was that three of four bolts sheared off at the end effector joint sensor. This was due to excessive friction torque transferred between the joint pin and joint sensor. The bolts were replaced and the joint was greased. This was completed in 2 hours. Lastly, a series of blown fuses on the excavator caused a $1 / 2$-hour outage. The total time for unscheduled maintenance was 4.5 hours. These unscheduled maintenance repairs were due to the fact that the equipment was prototype and had not been tested before. There were no scheduled maintenance outages because all scheduled maintenance and fueling was done before the demonstration start time.

Although there were no scheduled maintenance outages, in a production model for use in a TRU environment it is recommended that all IEE pin joints be greased on a regular basis by an automatic greasing system or that a maintenance free design be developed. All grease fittings are accessible and easy to maintain for standard operations. See Section 10.6 for scheduled maintenance time estimates. During Phase II, some routine maintenance was performed, including greasing the IEE pin joints, and daily fuel filter changes for the TTV diesel engine (1/2 hour).

7.1.2.4 Conclusion. In conclusion, the IEE overall system performance for spillage, ability to handle waste forms, visual estimate of dust generation, reliability, ease of use, ease of maintenance, volume of waste removed per excavation cycle, and rate of removal was deemed a success in the Phase I demonstration. The IEE system is an effective and reliable system for removing waste forms and transporting them through the entire excavating process. The IEE is rugged and suitable for digging all kinds of buried waste and can achieve a removal rate of $80 \mathrm{yd}^{3} /$ day. The Phase I demonstration successfully proved that the concept of eliminating the dumping at the digface is a viable, immensely practical approach to limiting potential contamination spread during buried waste retrieval. The IEE was easy to use and maintain. Overall, the Phase I demonstration and the performance of the IEE were extremely successful.

\subsubsection{Effectiveness on Various Waste Forms}

The data quality objective for this portion of Phase I was to document the various waste forms encountered and the ability of the IEE to move these objects through the entire excavation and transport process. 
7.1.3.1 Data and Data Reduction. For the Phase I demonstration, the specific waste forms were; INEL soil, paper, steel beams, 55 -gal metal drums, and 2-ft diameter $\times 8$-in. thick concrete rounds. Figures 10 and 11 show the test bed layout and the contents of the test bed. Appendix G gives a complete description of the types of waste forms removed during each cycle of each test. All data collected were qualitative and were recorded in the logbooks.

7.1.3.2 Data Analysis. No data analysis was required. Results on the effectiveness of the IEE for retrieving the various waste forms are given in Section 7.1.3.3 below.

7.1.3.3 Results. The results of the Phase I tests for evaluating the waste forms that the IEE could handle are given below.

A. INEL Soil

The INEL soil did not produce any difficulties for the IEE during the excavating or the transporting process. At first, however, the IEE's ability to retain a full load was restricted by the size of the overflow opening. This problem was corrected by restricting the size of the overflow slot as described in Section 7.1.2.3,C.

The IEE entered the digface cleanly without generating dust, but when exiting the digface there was some dust generation. When the IEE completed the dig, the operator would give the IEE a shake to clean off excess debris. This was also a source of dust generation. For an actual retrieval, contamination control at the digface will be essential in limiting the dust generated at the digface. No visible dust was generated when moving the IEE from the digface to the WTC. Only small amounts of dust were created when placing the ITM into the WTC. This dust was primarily caused by soil catching on the edges of the teeth and/or bucket and falling off when the ITM transfer was being done. Any spillage generated at this point was contained inside the envelope of the WTC. No dust was generated when moving the IEE to the ITM loading area, but light dust was created when mating the IEE to the empty ITM. Overall, the IEE was effective in handling soil and in not generating and spreading dust, especially at the transfer site.

B. Paper

Excavation of paper had nearly the same characteristics as the INEL soil, with the following exception. The IEE was originally equipped with ripper teeth. These ripper teeth would cause paper to hang up onto the IEE and potentially drop off while swinging the excavator boom. The teeth were changed to a grader bar as described in Section 7.1.2.3, C, which reduced this problem.

Once modified, the IEE was effective in excavating and transporting paper. At times, loose paper would hang up on the edges of the IEE, and fall off during placement of the IEE into the WTC or when mating to an empty ITM. However, once the paper was loaded into the ITM and compressed, it remained in place and did not contribute to spillage. Generally, the IEE did well in excavating paper. 


\section{Steel Beams}

There were two different lengths of structural steel used in this demonstration. There was one 7-ft long piece and 15, 3-ft long pieces. The IEE was used to pull the 7-ft beam from the waste pile and place it in the WTC. The IEE thumb was dexterous enough to grip the beam, extract it, and place it in the WTC. During this process, dust was generated at the digface when picking up the beam (primarily because of loose soil that rested on the beam and the general disruption of the waste pile), and no dust spread or spilled when transporting the 7-ft steel beam to the WTC. The 3-ft pieces of steel were easily placed inside the ITMs. This was done by using the thumb or by actively capturing them while digging. Dust was generated when the 3-ft pieces dropped from the thumb into the ITM.

D. 55-gal Drums (Barrels)

Initially, as the IEE dug into the digface, drums were being breached, which sent out a large plume of dust. The plume was believed to be primarily Portland cement dust from the dry Portland cement in the drums. There were three factors that contributed to the breached drums: the ripper teeth, operator inexperience, and the fact that the operator's view of the digface was obstructed by the ITM. The ripper teeth were replaced as defined in Section 7.1.2.3, C. Through operator experience, a new method for retrieving drums was discovered. This method was to detach the ITM and use the IEE to organize the digface to expose the drums. With the ITM removed, the operator had an unobstructed view of the drums and could grab them with the thumb and place them into an empty ITM located on the floor. Light dust was generated at the digface and at the ITM, but as a result of this second method, no further drums were breached. It was shown that the ITM is able to easily hold two 55-gal drums at a time.

E. Cement Rounds

Nearly all of the cement rounds were excavated with a mixture of paper and dirt. The cement rounds did not create any difficulty during excavation. The operator did show the dexterity of the IEE by using the thumb to pick a cement round out of the pile and place it on the floor. The operator then picked another cement round out of the pile and placed it onto the first cement round. Finally, the operator picked both cement rounds up together and placed them into an empty ITM.

7.1.3.4 Conclusion. The IEE was effective in handling all of the waste forms. The thumb was shown to be effective and dexterous at handling the various waste forms. When an object such as a drum fell from the thumb or digface into the ITM, it created some visible dust at the digface, however, it was somewhat contained by the IEE. Dust generation at the transfer location was minimal. The various objects were successfully retrieved by the IEE and transferred to the transfer station. None of the objects were dropped and only a small amount of spillage occurred.

\subsubsection{Overall Phase I Demonstration Success}

The test plan for Phase I states that Phase I could be deemed a success if sufficient data and operations experience were gained to assess the capability of the technology to accomplish its goals and if 
Environmental Restoration was provided with data to perform Feasibility Studies. The determination was to be based on the following:

- Collection of sufficient quantitative data to make a determination on the spread of contamination resulting from the application of the equipment and processes.

- Collection of sufficient quantitative data to make a determination on the amount of dust generation resulting from the application of the equipment and processes.

- Observations regarding overall system performance including spillage, ability to handle the waste forms, visual estimate of dust suppression, reliability, ease of use, ease of maintenance, and any other operation parameters are made and recorded for future reference.

7.1.4.1 Results. This report provides Environmental Restoration with technological data to perform Feasibility Studies. Sufficient data were gathered supporting this test to provide Environmental Restoration with data. Contamination control data are discussed in Section 7.1.1; operational data, in Section 7.1.2; and waste form handling, in Section 7.1.3.

7.1.4.2 Conclusion. The Phase I demonstration was extremely successful, not only in terms of data collection, but in terms of performance of the IEE. The dust and contamination spread testing showed that the IEE is effective in controlling the generation and spread of dust at the dumping or transfer location. As with any excavation not using contamination control methods, dust was generated at the digface. The average dust level was below the 1.1E-4 test goal.

The IEE system is an effective and reliable system for removing waste forms and transporting them through the entire excavating process. The IEE is rugged and suitable for digging all kinds of buried waste. The IEE was effective at handling the various waste forms present in the demonstration. In particular, the flexibility of being able to release the ITM and work with the IEE thumb to pick up objects and place them into the ITM or to move items around on the digface added to the ability of the system to handle a large variety of waste forms. The IEE thumb and design were found to be dexterous and capable of handling waste forms ranging from 55-gal drums to loose paper and soil. The overall system integration between the REMEX, IEE, ITM, and WTC was excellent and added to the smooth and successful completion of the Phase I demonstration.

\subsection{Phase II Demonstration Test Results}

\subsubsection{Throughput}

The data quality objective for this portion of Phase II testing was to document the rate of waste removal and transport as performed during the demonstration by measuring the time of actual excavation operations and number of cubic yards of soil removed and conveyed. A goal of $80 \mathrm{yd}^{3} / \mathrm{day}^{\text {in }}$ an 8 -hour shift was used for design of the demonstration system. The throughput tests included all types of waste that were encountered during tests (i.e., waste that fit into the ITM and large objects). An ITM holds a maximum of approximately $1.4 \mathrm{yd}^{3}$ of simulated buried waste mixed with soil.

7.2.1.1 Data and Data Reduction. Phase II consisted of three tests. A summary of the average excavator cycle time, average conveyance cycle time, average total cycle time, average volume of waste 
in the ITM, and a production rate is given in Table 7. A more complete description of test results can be found in Appendix $\mathrm{H}$.

The total volume and times were recorded for each cycle. Time was recorded to the nearest second using a digital watch. The accuracy of the time measurement is estimated to be \pm 1 second.

The volume of waste removed per excavation cycle was estimated and recorded in the logbooks for each cycle. A conveyance cycle is the time it took for the TTV to travel from the loading station to the unloading station, deliver a full ITM, receive an empty ITM, and travel back to the loading station. An excavator cycle is the time it took for the REMEX to deposit a full ITM and receive an empty ITM, not including any digging or actual excavation time. The digging cycle took place simultaneously to the conveyance cycle and times were not recorded because the data would be redundant to data collected during Phase I testing. The volume of waste was estimated by using volume markers inside the ITM. These markers were at $1 / 2,3 / 4$, and $7 / 8$ of a full ITM $\left(1.4 \mathrm{yd}^{3}\right)$. The measurement was recorded by visually looking at the nearest marker and estimating the volume that was above or below the marker. The estimated accuracy of the volume measurements is $\pm 1 / 8$ of an ITM or $0.175 \mathrm{yd}^{3}$.

The average production removal rate for each test was obtained by adding the total volume retrieved and dividing that volume by the total time it took to perform those cycles.

7.2.1.2 Data Analysis. The data in Table 7 represents the averages from each test. The complete data set is given in Appendix H. The data were recorded for each cycle for each test. Each test represents the average of all of the cycles for that test. The first test was not included in the analysis because there were insufficient data.

Again, the rate of removal was calculated by dividing the volume retrieved by the time it took to retrieve that volume. The calculation for the rate of removal did not include time-outs for unscheduled maintenance or precision and accuracy measurements. The rate of removal was calculated by extrapolating the actual rate of removal into an 8-hour day. For instance, if the actual demonstration time for 1 day was 4 hours, the actual rate of removal during that period was doubled when considering the achievable throughput rate for an 8-hour day.

7.2.1.3 Results. There were several key contributors to the production rates obtained during the demonstration: operator proficiency, TTV speed, excavator vision system, and manual dumping operation at the docking station.

The excavator operator easily adapted to the remote control system for excavating the waste. This learning process was accelerated by the in-cab experience gained during Phase I of the demonstration. The excavating cycle was done in parallel with the conveyance cycle. The conveyance cycle time was lengthened by the reduced TTV speed and the increased path length, so the excavation cycle was completed well in advance of the conveyance cycle.

The TTV operator learned rapidly, navigating the TTV exceptionally well. First of all, the operator had to become familiar with the TTV travel path by using the TTV onboard vision system, which proved to be inadequate because of the narrow field of view and slow pan and tilt camera functions. The lack of 
Table 7. Summary of production rates and equipment cycle times for Phase II testing.

\begin{tabular}{|c|c|c|c|c|c|}
\hline Test date & $\begin{array}{c}\text { Average } \\
\text { excavator } \\
\text { cycle time } \\
\text { (min) }\end{array}$ & $\begin{array}{c}\text { Average } \\
\text { conveyance } \\
\text { cycle time } \\
\text { (min) }\end{array}$ & $\begin{array}{l}\text { Average total } \\
\text { cycle time } \\
\text { (min) }\end{array}$ & $\begin{array}{c}\begin{array}{c}\text { Average } \\
\text { volume in ITM } \\
\left(\mathrm{yd}^{3}\right)\end{array} \\
\end{array}$ & $\begin{array}{c}\text { Production } \\
\text { rate } \\
\left(\mathrm{yd}^{3} / \text { day }\right) \\
\end{array}$ \\
\hline $9 / 27 / 94$ & \multicolumn{5}{|c|}{-Insufficient data for average calculations__a } \\
\hline $9 / 28 / 94$ & 3.13 & 9.51 & 12.81 & 1.30 & 48.71 \\
\hline $9 / 29 / 94$ & 2.61 & 8.51 & 11.26 & 1.02 & 43.48 \\
\hline Average & 3.30 & 9.38 & 12.68 & 1.20 & 45.42 \\
\hline
\end{tabular}

an overview camera view impaired the TTV navigation as well. Secondly, the operator had to gain experience in using the vision system and the ultrasonic distance sensors to align the TTV to the targets at each docking station. Finally, the operator had to learn to coordinate with the excavator operator during the ITM load and unload cycles. Once the TTV operator gained experience in these areas, the average conveyance cycle time was reduced by 1 minute as shown in Table 7.

The calculated throughput was a little more than one half of the $80 \mathrm{yd}^{3} / \mathrm{day}$ goal as described in the test plan. There are three reasons for this reduction in throughput. First, the TTV engine horsepower was not adequate. Second, the TTV track hydraulic flow was reduced by $50 \%$; as a result of this reduction, the TTV travel speed was reduced by $50 \%$. This hydraulic modification was done to compensate for the lack of engine power when the TTV was attempting turns and to improve the TTV maneuverability. (See Sections 9 and 11 for more details on lack of engine power and proposed modifications.) Third, the TTV travel path was $100 \mathrm{ft}$ longer than the original test plan specified path length, increasing the conveyance cycle time. With the appropriate horsepower engine, original hydraulic flow rates, and the path length prescribed in the test plan, ${ }^{4}$ the $80 \mathrm{yd}^{3} /$ day goal should be easily achievable.

The excavator vision system consisted of four video cameras. These cameras were positioned as follows; one camera was mounted on the arm of the excavator for a view of the IEE, one camera was mounted on the excavator cab, one camera showed a right angle view of the digface, and one camera showed a right angle view of the TTV positioned for load and unload of the ITMs. The right angle cameras proved to be the most effective because they gave the operator depth perception, which helped reduce the excavation and load/unload cycle times. The data in Table 7 show that as the operator became more proficient at using the vision system, the cycle times decreased. Note that the excavator cycle time consisted of loading and unloading the ITMs from the TTV. When operating the excavator manually in the cab, the operator was able to reduce the excavator cycle time by 1 minute over remote operations. The excavator operator indicated that remote operations were preferred over in-cab operations because of the improved communication with the TTV operator and because the right angle camera views provided a better view of the digface than the in-cab perspective.

A fork lift operation was used to manually load and unload the ITMs at the TTV docking station. This method is not indicative of an actual production operation. The average estimated load and unload 
cycle time was 2 minutes, which was included in the conveyance cycle time. A different load and unload method would help decrease the conveyance cycle time and increase the production rate.

7.2.1.4 Conclusion. The throughput tests for Phase II included the total cycle time for both the excavation and conveyance tasks. The calculated throughput was $45.42 \mathrm{yd}^{3} / \mathrm{day}$, which is lower than the $80 \mathrm{yd}^{3} /$ day (8-hour shift) throughput goal as defined in the test plan. There was not a significant difference in excavation cycle times for in-cab versus remote operations. The calculated average throughput from the test data includes operator increased cycle time due to inexperience, decreased TTV speed due to insufficient horsepower and corresponding hydraulic flow rates, and an increased path length. With corrections to these areas, it is expected that the system would easily meet the objective of $80 \mathrm{yd}^{3} / \mathrm{day}$, and could easily achieve 80 to $130 \mathrm{yd}^{3} / \mathrm{day}$. In addition, should greater throughput be desired, the TTV speed can be increased, and/or additional vehicles could be used. A detailed discussion of modifications that could be made to increase the throughput can be found in Section 11.

\subsubsection{Effectiveness on Large Objects}

The data quality objective was to document the capability of the IEE to handle a variety of large objects (large being greater than a 55-gal drum) and to document the number and type of large objects dropped and the amount of slip for objects successfully moved.

7.2.2.1 Data and Data Reduction. For the demonstration, the specific large objects were one truck bed, two 15-ft lengths of structural steel, and two 15-ft lengths of pipe ( 8 and 12 in. diameter). All data collected were qualitative and were recorded in the logbooks. Note that the large objects discussed here were not included in Phase I testing.

7.2.2.2 Data Analysis. No data analysis was required. Results of IEE retrieval of various waste forms are given in Section 7.2.2.3 below.

7.2.2.3 Results. The results of IEE effectiveness in handling large objects are given below.

\section{A. Truck Bed}

There were no difficulties in retrieving the truck bed out of the digface. The IEE and thumb successfully grabbed the truck bed without dropping or slippage and then transported it to the side of the excavation area. The IEE was then used to compact the truck bed into a size suitable for transporting in the WTC. The IEE successfully loaded the compacted truck bed into the WTC as shown in Figure 27.

\section{B. Fifteen Foot Lengths of Steel and Pipe}

Once again, there were no significant difficulties in retrieving and transporting the structural steel or the pipes. The IEE was effective in handling these large objects without dropping or slippage. However, the front shovel configuration used did impact the operator's visibility of the digface, especially from in-cab operations. This affected the operator's ability to see what the thumb was grasping, and at times created some dust at the digface. 


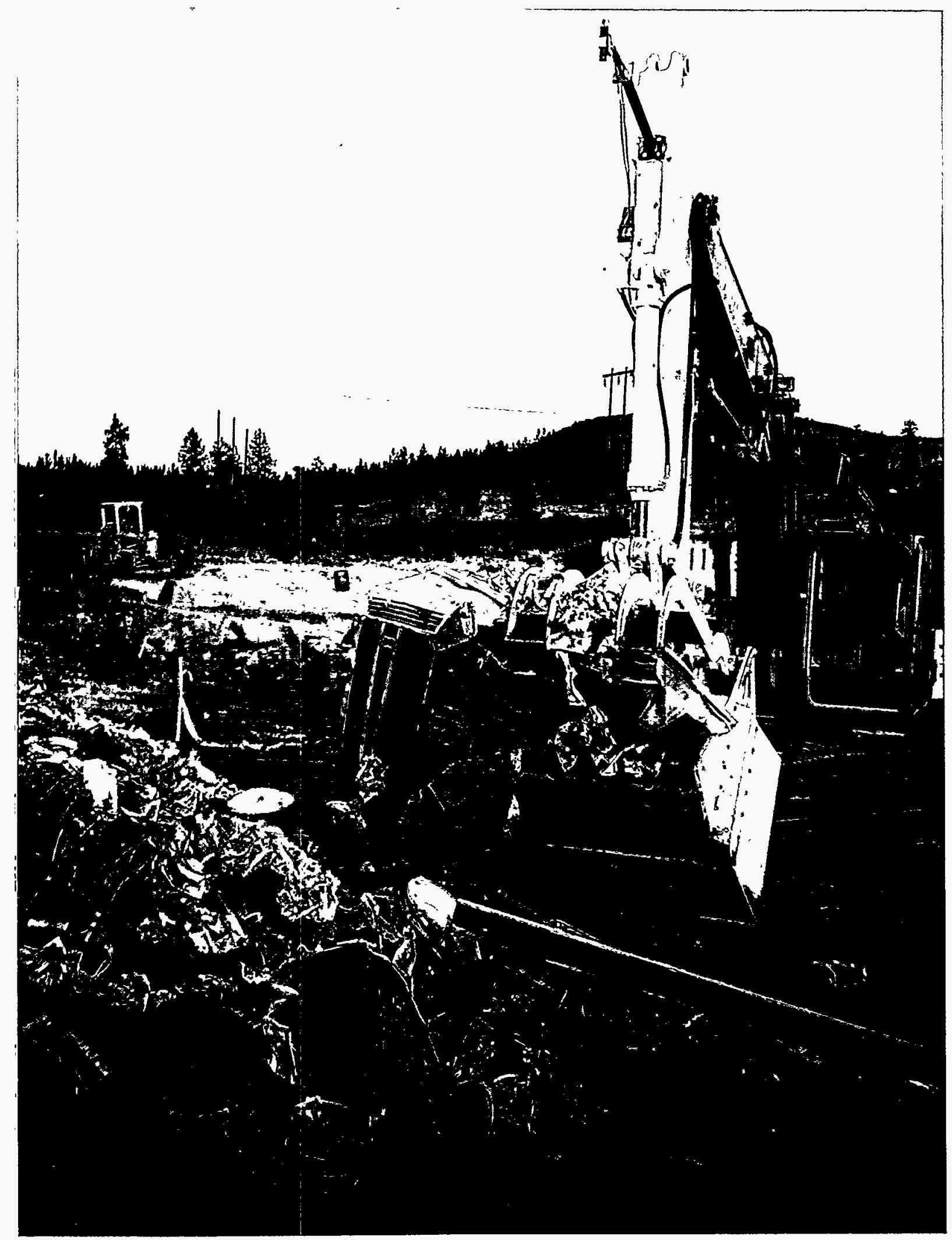

Figure 27. The pick-up truck bed was manipulated to size it to fit into the WTC using the IEE (INEL 94-1187-12-23). 

7.2.2.4 Conclusion. The IEE and thumb were extremely effective in handling and transporting the large objects from the digface: This operation was completed without any drops or slippage of the large objects. However, the front shovel configuration used had an affect on the operator's visibility of the digface. With a backhoe configuration visibility would be improved, as would the operator's ability to use the thumb to grasp objects with less dust generation.

\subsubsection{Effectiveness on Various Waste Forms}

The data quality objective was to document the various waste forms encountered and the ability of the system to move these objects through the entire excavation and transport process.

7.2.3.1 Data and Data Reduction. The specific waste forms used were the same ones used during the Phase I testing. The large objects discussed in Section 7.2.2 were not included in the Phase I testing and were added for Phase II testing. See Section 7.1.3.1 for a complete description of the waste forms. In addition to these waste forms, four 50 - $\mathrm{ft} \times 1 / 4$-in. diameter wire ropes were added to the waste pile for Phase I testing. All data collected were qualitative and were recorded in the logbooks.

7.2.3.2 Data Analysis. No data analysis was required. Results of the IEE for retrieving the various waste forms are given in Section 7.2.3.3 below.

\subsubsection{Results.}

A. Waste Forms From Phase I Testing

These waste forms were handled without difficulty. See Section 7.1.3 for a detailed discussion of the IEE's ability to handle these waste forms. The ability to transport these waste forms was excellent. The TTV had no trouble transporting the loaded ITMs to the dumping station and there was no spillage during the transportation process.

B. Wire Rope

The wire rope proved to be more difficult to handle than the other waste forms. The IEE could extract the rope from the digface, but could not completely contain the rope in the ITM. This made it difficult to load the ITM into the WTC. Through experience the operator found that it was easier to detach the ITM and use the IEE to load the rope into the ITM. The IEE and the thumb were used to thread the rope into the ITM. This excavation option demonstrates the flexibility of the IEE/ITM system. This flexibility provides a significant advantage over standard bucket configurations and expands the range of tasks and waste forms that the IEE can successfully retrieve and prepare for conveyance away from the digface.

7.2.3.4 Conclusion. The capability of the system to handle the various waste forms is excellent. The IEE and TTV integrated well and there was no spillage when loading and transporting the waste. Although the wire rope was more difficult to handle, with operator experience and the flexibility of the system, it became manageable. Operator procedures and approaches such as detaching the ITM to see the digface and place objects into the ITM were facilitated by the system's flexibility and enhanced the system's ability to handle waste forms and reduce dust generation during retrieval operations. In addition, other improved operator procedures and approaches to using the system's flexibility should enhance contamination control and safety even further during retrieval operations. 


\subsubsection{Effectiveness of Telerobotic Control System}

The data quality objective for this portion of Phase II was to document the capabilities of the remote control system and the ability of the system to effectively control the remote conveyance system by photographs, videotape, logbook entries, and operator interview records. To document the capabilities of system, the following were demonstrated and documented: the ability of the system to effectively control the remote conveyance system, evaluation of the telemetry system (e.g., range and signal strength), vehicle speed, vision system, human factors aspects, collision avoidance system, reliability, and safety.

The REMEX was not part of the original scope of work and was a value-added feature, so there were no test objectives regarding the REMEX. Although the REMEX was not part of the tests, observations regarding the performance of the REMEX are discussed throughout Section 7.

7.2.4.1 Data and Data Reduction. Details on the data and data reduction for the telerobotic control system are given below.

A. Ability of the System to Effectively Control the Remote Conveyance System

The information in this section is primarily based on TTV operator and design team observations.

B. Evaluation of the Telemetry System (e.g., range and signal strength)

The telemetry range test was conducted by starting the TTV at the remote control station and then driving a distance of $500 \mathrm{ft}$. At set intervals of 10 seconds, the distance traveled and number of packets of data received were recorded. Two out of 28 data points were missed and not used in the analysis. The time it took to travel the $500 \mathrm{ft}$ was 280 seconds. The number of packets sent was consistent over the entire range. The system is not synchronized, so a set number of packets per time period is not expected. The data gathered were whether a consistent amount of data were received over the entire telemetry system range.

C. Vehicle Speed

Vehicle speed was determined by remotely driving the TTV a certain distance and dividing that distance by the time it took to traverse that distance. The distance the vehicle was remotely driven was $100 \mathrm{ft} \pm 1 \mathrm{ft}$. Time was measured using a digital watch and had an uncertainty of \pm 1 second. The test was repeated two times traveling forward and two times traveling reverse.

D. Vision System

The effectiveness and capability of the vision system was measured qualitatively throughout the demonstration, with observations recorded in the logbooks. In addition, qualitative data on the range of the vision system was taken during the telemetry test (see Section 7.2.4.1, B).

\section{E. Human Factors}

The information included for the human factors evaluation is based primarily on operator feedback and observations of the test team. 
F. Collision Avoidance System

Informal testing of the collision avoidance system was done, and it was found that the completion of this test was not feasible because of difficulties with the ultrasonic sensors. Crosstalk between the ultrasonic sensors led to erroneous readings and would have prevented the vehicle from avoiding obstacles as designed.

G. Reliability

The number of loading/unloading cycles completed without human intervention was noted throughout the demonstration and recorded in the logbook.

H. Safety

The effectiveness of the safety systems was tested before the start of the demonstration during a performance test. Items tested included the supervisor's belt module RF emergency stop, vehicle on-board $R F$ emergency stop, remote control station hydraulic stop (emergency stop), motion warning siren, and engine active flashing light signal. In addition to the performance test, qualitative data on the performance of the safety features were taken throughout the demonstration and recorded in the logbooks.

7.2.4.2 Data Analysis. Data analysis information for the telerobotic control system is given below.

A. Ability of the System to Effectively Control the Remote Conveyance System

No data analysis required. The results are presented in Section 7.2.4.3.

B. Evaluation of the Telemetry System (e.g., range and signal strength)

Since the TTV sends signals at a continuous frequency, the number of lost packets of information was inferred from the number of good signals received.

For items C, Vehicle Speed; D, Vision System; E, Human Factors; F, Collision Avoidance System; $\mathrm{G}$, Reliability; and $\mathrm{H}$, Safety, no data analysis required. The results are presented in Section 7.2.4.3.

7.2.4.3 Results. Results for the effectiveness of the telerobotic control system are given below.

A. Ability of the System to Effectively Control the Remote Conveyance System

TTV Piloting

During the first week of operation, the TTV was tuned to provide a straight-line speed of approximately $300 \mathrm{fpm}$ when the tracks were unloaded. During tuning, it was noted that the left track required larger electrical signals to both the forward and reverse proportional solenoid valves to achieve the same track speeds as the right track (the right track is the one on the same side as the engine). It has not been determined whether this disparity is caused by the mechanical condition of the tracks, hydraulic track motors, hoses, shuttle valves, or solenoid valves (differences in return spring characteristics, relief valve settings, etc.). 
Using pendant control, there was an estimated control lag of approximately $200 \mathrm{~ms}$ (this was not confirmed with a stopwatch) from control input to vehicle response. Under remote control, however, the control lag was almost doubled (once again, this was not verified with a stopwatch).

It was recognized early on that the 47 horsepower engine was not enough to easily turn the vehicle. Steps were taken to improve vehicle-turning including increasing the hydraulic pressure to $3,500 \mathrm{psi}$, reducing the hydraulic flow, and modifying the grousers on the vehicle tracks. All possible combinations of proportional valve settings for both tracks were attempted, and it was found that the vehicle turned best when the contact patch between the tracks and the ground was smallest, such as when the TTV was on a low mound of sand. This was true regardless of the speed of the vehicle. Each time the TTV commenced a turn, the tracks would plow sand that would oppose any further movement in that direction. The engine was set at the maximum operating speed to try to overcome these problems, but the power required to turn would stall the engine. Eventually the demonstration was performed at a higher pressure setting on the hydraulic pump but with a much lower flow rate to compensate. With more available torque on the track motors, the TTV turned more readily but traveled at a much-reduced straight-line speed. The grousers on the vehicle tracks were modified to improve maneuverability as described in Section 7.2.5.

The control mode of the vehicle affected the effectiveness of the control system to control the vehicle. The following comments refer to operation on a level surface:

Under direct track control, the tracks are controlled in an open-loop manner. The open-loop gains were, at first, set low to avoid stalling the engine under certain conditions. This control mode revealed the mismatching of the left and right tracks; the left track would always move faster than the right track, unless they were both stalled in the sand.

Under robotic track control, each track is controlled in a feedback loop. A track whose actual velocity lags its desired track velocity (determined by joystick commands) will result in a larger signal to the corresponding proportional valve to match the desired track velocity. Even when the maximum allowable gains were set very high for both tracks, the right track still lagged behind the left track in straight-line motions of the TTV. This velocity mismatch, although somewhat less severe than in direct track mode, was apparent regardless of the TTV velocity.

The tracks did not provide equal power for locomotion or turning. The left track provided less power than the right track. The cause of this problem is not known to date but is probably a combination of control system tuning, hydraulic imbalance, and mechanical resistance in the tracks. With this power imbalance, it would be expected that the vehicle would be able to turn to the right but, perhaps, not to the left. In fact, the opposite occurred; when stationary, the vehicle was unable to turn right but able to turn left. In considering the vehicle weight distribution, this can be explained as follows. For left turns, the left track has enough torque to move backwards, and the right track is able to move slowly forward to keep from digging in. For right turns, the left track has enough torque to move forwards, but the right track cannot stay in motion due to the concentration of weight on the right side of the vehicle, so the TTV is 
unable to turn right. For straight line travel, the TTV tends to veer to the left when traveling forward and backwards due to the weight distribution and track power imbalance. It is expected that all of these problems might be unnoticeable if the vehicle horsepower were adequate.

The TTV did not turn right with both tracks moving in the same direction, no matter what forward or reverse speed. The only time that a turn could be made is when the inside track was given a command to reverse its track direction. A left turn, however, could be made at a slow angular acceleration (on the order of $1 \mathrm{deg} / \mathrm{s}$ ) when both tracks were traveling in the same direction. Regardless of the desired turning direction, the inside track would move almost as fast as the outer track, as long as its corresponding proportional valve was open enough to allow flow to the track motor. Consequently, the only effective way to maneuver the TTV was to send it on paths made up of polylines. The TTV traveled in straight lines, and each time a target or a landmark was reached, the joystick is deflected in an attempt to spin the TTV about its axis. Each turn was made with full control deflection to take advantage of the TTV's angular momentum; a partial joystick deflection often left the TTV short of its desired heading from plowing up sand on its tracks.

B. Evaluation of the Telemetry System (e.g., range and signal strength)

The telemetry system is a spread spectrum system operating between 902 and $928 \mathrm{MHz}$. The system was tested throughout the demonstration, and a performance test of the signal strength was conducted.

During the telemetry test, the amount of signal degradation was gauged by the change in value of signal received. It was found that there was no degradation of signal (i.e., no loss of data packets received) over the 500-ft range. As a double check, the number of errors (data packets received that were corrupt or unreadable) was monitored as the vehicle returned to the control station. This information confirmed outgoing data indicating no loss of signal over $500 \mathrm{ft}$.

C. Vehicle Speed

During the first week of operation, the TTV. was tuned to provide a straight-line speed of approximately $300 \mathrm{fpm}$ when the tracks were unloaded. The TTV achieved a measured speed of about $240 \mathrm{fpm}$ on the ground with neither a container nor an ITM on board. The vehicle speed was later reduced by the reduction in horsepower that provided more torque for turning and improved maneuverability (see Section 7.2.4.3, A). The speed was not measured with the reduced horsepower.

D. Vision System

\section{On-Board Cameras}

The TTV cameras have 1/3-in. CCDs with a 6 to $60 \mathrm{~mm}, 10 \mathrm{X}$ zoom, and auto-iris lenses. When set at the widest angle, this combination produces roughly a 30 degree view cone. This narrow field of view limited the effectiveness of the TTV cameras. The focus controls were seldom used because the focus is automatically set at infinity when the zoom lens is at the widest angle setting. 
A bent piece of threaded rod was attached to the base of the pan and tilt units to provide a pan reference to the camera. When used in conjunction with the video overlay generator that created the crosshairs on the video display, it was relatively easy to pan the camera so that it points along the longitudinal vehicle axis.

The on-board camera views were not as useful for navigation as anticipated because of the narrow field of view and slow pan and tilt functions. The pan and tilts were used extensively at first to survey the terrain and the TTV's orientation on the path. The pan and tilt functions were slow, which limited the usefulness of the TTV cameras. After several circuits, however, it was found that it was more convenient to follow the TTV's old tracks on the ground, rather than repeating the time-consuming pan and tilt operations to confirm the TTV's location. In addition, when the REMEX was not busy, the REMEX operator raised the boom on the REMEX to provide an overview of the test site on the REMEX control station display. This overview screen was helpful in navigating the TTV along its path.

For the most part of the final days of testing, the cameras were set to point along the longitudinal axis of the vehicle and slightly down so that the reference rod was barely visible at the bottom center of the screen. The zoom lenses were also left at the widest angle setting.

The cameras provided a relatively high-resolution image, with good color reproduction to help in terrain recognition. The auto-iris performed well in all light levels.

\section{Wireless Video}

The wireless video system is a low-power system that is able to transmit a video and audio signal at up to $250 \mathrm{ft}$ at a frequency of $2.4 \mathrm{GHz}$.

The video transmitter was mounted on a sheet of 0.125-in. metal, with a custom-shaped plastic dome to protect the unit from splashing rain and dust. A 1.0-in. vertical pipe was also mounted about $1 \mathrm{ft}$ away from the centerline of the transmitter. This pipe was used to secure the $900 \mathrm{MHz}$ telemetry antenna and the $150 \mathrm{MHz}$ RF Emergency Stop antenna about $2 \mathrm{ft}$ above the video transmitter unit.

The video receiver was mounted on a wooden platform placed on top of the trailer, with only the $900 \mathrm{MHz}$ base station telemetry antenna mounted on the same platform. The telemetry antenna was mounted about $1 \mathrm{ft}$ horizontally and $1 \mathrm{ft}$ vertically away from the video receiver unit, using a thin sheet metal bracket provided with the base station antenna.

During the telemetry test, the range of the video system was evaluated. The video system range was $250 \mathrm{ft}$, so it was expected that the signal would not carry over the 500 -ft telemetry test range. The video signal degraded at $200 \mathrm{ft}$ and was completely lost at $246 \mathrm{ft}$. However, the video signal returned at various times from 250 to $500 \mathrm{ft}$, which was probably caused by signal reflections. Since the vehicle path was straight and radio contact was maintained, safety was not compromised while running the vehicle without video signals. 
When the TTV was within about a 200 -ft radius of the receiver, the video signal was crisp and clear most of the time. Occasionally snow appeared on the screen. In addition, the video signal became almost unusable at a certain orientation of the TTV. This was probably due to interference from the antenna mast. The video signal was only slightly cluttered with snow when the vertical pipe (antenna mast) on the TTV was aligned anywhere between the video transmitter on the TTV and receiver on the trailer. However, the video signal was noisiest when the pipe was' aligned perfectly on the far side of the video transmitter on the TTV, and also when the TTV was brought out of range from the receiver. The cause of this has not been determined but could be caused by the signal reflecting off the pole and coming back to the antenna in such a phase as to cause cancellation of the desired signal.

\section{Remote Control Station Displays}

It is helpful to be able to see the TTV from a view other than the ones provided by the cameras on the TTV. When loading an ITM from the excavator, the view from the excavator cab-mounted camera was the most effective in aligning the TTV with the ITM for placement into the WTC. This camera also provided confirmation of the lid/latch status lights on the TTV remote station.

The video targets were helpful in confirming that the TTV was perpendicular to the video target. At a distance, lining up the TTV involved aiming for a point on the far side of the video target, driving for this point, and turning onto the target when the TTV was within $15 \mathrm{ft}$. At closer distances, alignment could be made only through small movements until the video target pattern shown on the video monitor was satisfactory.

\section{E. Human Factors}

\section{Qualitative Evaluation of the Control Stations}

The control station equipment was built using existing equipment and was not designed specifically for human factors. However, wherever possible, the configuration took human factors design into account (see Figure 28). The television monitors were located at an appropriate height for operator viewing, and the controls were conveniently located. Adding swivel plates to the television screens would improve the station versatility as well as aiding the ease of communication between the two operators because they viewed each other's screens occasionally to get a better overview of the demonstration.

The control station was the only indoor location at the test site, so people tended to be inside the trailer. The operator concentration did not seem to suffer, but it would be preferable to have a separate indoor location for nonoperator personnel, or for personnel needing a break. In addition, a soundproof glass wall between visitors and operators would reduce distractions.

Two operators were used for the demonstration: one TTV operator and one remote excavator/IEE operator. In addition, a test director, two forklift operators, and several data recorders participated in the test. 



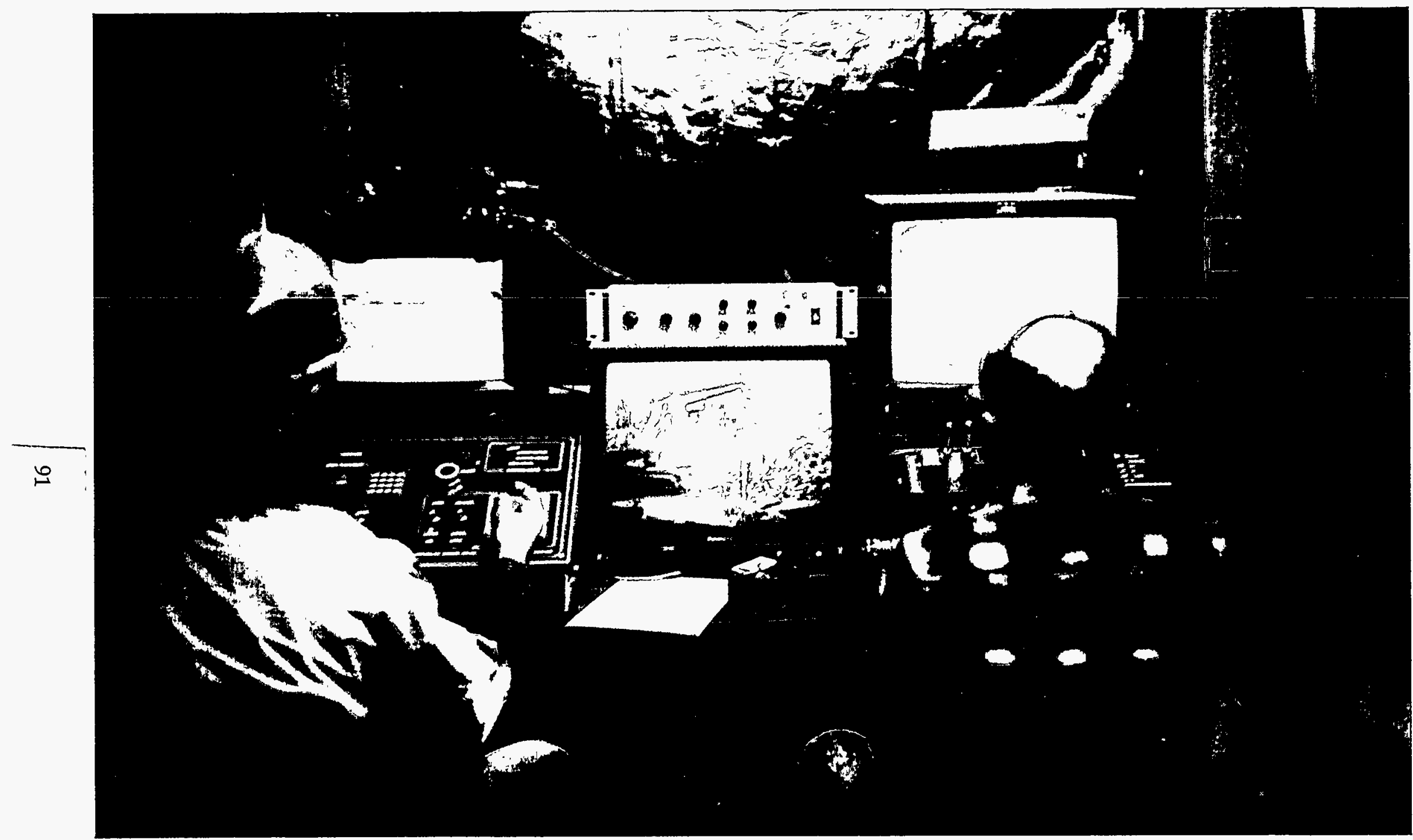

Figure 28. The remote control station employed existing equipment and considered human factors design as much as possible. The proximity of the remote operator control stations aided in operator communication and allowed the operators to use each other's camera views in lieu of an overview camera (INEL 94-1187-1-15). 
The excavator/IEE operator was experienced operating heavy equipment. When interviewed, the operator indicated that previous experience with heavy equipment was not particularly helpful in becoming adept at using the joystick control arm and in operating the excavator. It did appear that previous experience with a waste pit and handling the waste forms was an advantage for the operator. The excavator learning curve for Phase II was shorter than Phase I because the operator became familiar with joystick control arm while in the cab during Phase I. It has been recommended that all learning to run remote operations with remote control should begin with time in the cab using the joystick control arm.

The TTV operator had no previous experience with operating remote vehicles. The TTV operator was intimately familiar with the TTV control system design, which helped immensely with troubleshooting and interpreting the vehicle performance. The TTV operator had extremely good eye to hand coordination, as well as dexterity with joystick controls. These capabilities should be considered when selecting remote vehicle operators for future tests.

\section{Controls}

REMEX - The REMEX joystick operating system is much easier to learn than a standard excavator control system. Once the joystick operating system is learned, it does not provide much advantage over standard control configuration for an operator experienced with standard control configurations. However, it is helpful to be able to perform all operations with one hand. In the future, if the system is upgraded to use coordinated control where the computer assists with coordination of the motions, it is believed that this system would provide a significant advantage over standard operating systems. The joystick operating system could be improved by decreasing the length of stroke for each motion. Fine control would be easier to use.

The REMEX operator indicated that the hand grip in the remote control station was uncomfortable. The grip was too large, and the control arm in the cab with the trigger underneath was preferable. The coordinated control arm is a prototype and would need to be upgraded to an industrial system for production units.

A test was done to determine whether acoustic signals from the excavator position at the digface would aid in excavator remote operations. A radio that was set on a different frequency was placed in the excavator $\mathrm{cab}$, and the headset was given to the remote operator. It was determined that rather than aiding in remote operations, the acoustic signal distracted from the operator's concentration by providing information that was not necessary and did not contribute to the ability to dig the waste remotely.

The REMEX operator indicated a preference for using joint control over coordinated control (which was not available for this test). It appears that experienced operators tend to indicate a preference for maintaining control of exactly what the excavator will do relative to the operator's hand motions, rather than having a computer coordinate the motions to create the effect desired by the operator's hand motions. In general, it appears that experienced operators would usually prefer to have all of the control directly linked to their motions, while new operators would prefer to have computer assistance to coordinate the motions. However, it is 
expected that once coordinated control is available, the ease of operations with the computer assisting with aspects such as level crowd control may be preferable to all operators.

Conveyance system-The pocket in the WTC for the ITMs is effective because it self aligns and locks into place even if the alignment is slightly off. The ITM cradle on the TTV with springs and cables works extremely well. Without the spring bed and the flexibility it provides, loading an empty ITM would be extremely difficult.

As with the excavator, the motions of the joystick were too large and fine control would have been easier to use.

\section{Displays}

The displays for both systems aided operators in accomplishing waste retrieval, transfer, and transport. Figure 28 shows a picture of the operator stations for both the excavator system (right hand side of figure) and conveyance system (left hand side of figure). Below is a discussion of some general observations that apply to both vision systems. Following the discussion is information on the excavator system and conveyance system displays and vision system interface.

Glare from fluorescent lights in the control trailer as well as the opening door contributed to the video display inadequacies. One of the two fluorescent light bulbs was removed to reduce the glare. The location of the door was directly behind the video displays. Future control trailers should position the door so that opening and closing it does not add glare to the video displays and include a lighting design that reduces glare.

When the sun was at a low angle, the direct sunlight entering the fixed video cameras activated the auto iris function of the camera and the lens closed to compensate for the bright light. The lens closed to the point that the image was too dark to be able to distinguish what was being seen, effectively shutting down the demonstration for a period of time.

The alignment of the excavator/TTV was easier when the excavator was very close to the TTV; the TTV would then move forward and back to get the exact alignment for ITM transfer. It was easier for the TTV to move forward and back for exact alignment than for the REMEX to move left and/or right and maintain alignment with the WTC's ITM recess (i.e., if the REMEX swung the bucket to the right or left to match the TTV position, the ITM would no longer be parallel with the recesses in the WTC, so several motions would be needed to move the REMEX and IEE left or right, while moving the TTV was simply a forward/reverse motion. The alignment of the TTV and excavator for loading and unloading ITMs relied on close communication between the TTV and excavator operators, as well as sharing views from both video systems. The TTV operator often looked at the REMEX boom camera view on the REMEX operator's workstation during this alignment process.

REMEX displays-The REMEX vision system consisted of single views in the direction of the operation, as well as stationary cameras with right angle views of the digface. During testing, the REMEX operator was queried about the operator interface. The operator noted that more 
cameras would be helpful so different views and angles could be seen. The operator also expressed that the vision system needed higher resolution cameras to see the detail on the digface and reduce operator fatigue. In addition, the excavator cameras were black and white, which significantly affected the depth perception for the excavator operator. The lack of depth perception affected the operator's ability to efficiently excavate the digface. The operator found that by using the two right angle camera views, the depth of the digface could be seen. The operator indicated that using color cameras would provide additional cues to depth perception.

The REMEX camera system was not designed for outdoor use. The camera system should be a rugged, weather-ready system.

While operating the excavator from the cab, the ITM (bucket) obstructed the view of the digface so the operator could not see what the thumb was gripping. Adding a camera view in the cab would alleviate this problem. During the demonstration, for in-cab operations, the IEE without the ITM attached was used to manipulate the digface between ITM loads.

Conveyance system displays-The TTV pan and tilt functions on the cameras were only used the first few runs to learn the terrain. Once the operator was familiar with the path, the pan and tilt units were not used. The pan and tilt functions on the TTV cameras were slow, and the field of view was very narrow. These factors contributed to the little use of the TTV cameras. However, it also became apparent that the on board cameras are limited in their field of view because of their position on the TTV. Overview cameras would provide adequate detail once the TTV path was learned and would provide more information about the general position and direction of the TTV. Because there were no overview cameras during this demonstration, the TTV and excavator operators worked together and found that the excavator cameras could be used to obtain an overview picture by swinging the excavator boom to various positions. This overview picture was helpful to the TTV operator because it provided a view of the overall TTV position and direction relative to the work site. However, during an actual excavation using several TTVs, the excavator did not have time or perhaps the vertical latitude to provide this function. The operators noted that more cameras including overview cameras would be helpful so that different views and angles could be seen.

The TTV station would benefit from a dual screen so both the overview and TTV cameras could be viewed simultaneously.

\section{Communications}

The test director was in constant radio communication with both operators. Radios were also used by the data recorders and the person operating the emergency stop switch. All radios operated on the same channel, so that only one person can talk at a time, and the communication was heard by all radio wearers. Although the radios worked well, this system could be improved to improve safety. A system where the test supervisor has an override channel that can interrupt other conversations to deliver an emergency message would improve safety. 
Radio communications were essential to the safety and success of the demonstration. Only through radio link could the test director have communicated with the equipment operators to give emergency directions. The test director was equipped with a master kill switch to shut off the equipment, so safety was not jeopardized. However, a situation might arise where communication would be preferable to an emergency shut down, so the radio link is essential.

As mentioned previously, the operators compensated for the inadequacies in the video system to some degree by sharing video images and using the excavator camera to obtain an overview picture. Verbal communication was also essential, which was aided by a relatively quiet environment in the control trailer.

\section{Environment}

The dimensions of the control station trailer were $11 \times 20 \mathrm{ft}$. An air conditioner was used for cooling when needed. No heating was available, and it was not required. The air conditioning unit successfully reduced humidity in the control trailer when many people were in the trailer. The trailer was lit by an overhead fluorescent light fixture which, as discussed previously, added to the undesirable glare on the video display terminals. The trailer had three windows which were covered completely to eliminate light and prevent personnel from viewing the test. The operators' chairs were ergonomic office chairs that fit well with the control workstation. The trailer was carpeted, which added to the noise reduction within the work area. However, the noise from the generator was audible in the trailer. The operator's workstation was positioned at one end of the trailer, with both control stations side by side. This aided in communications between the operators. The operators were positioned with their backs to the door, which reduced the distraction of other people entering the trailer.

F. Collision Avoidance System

Due to crosstalk between the ultrasonic sensors, the test of the collision avoidance system could not be completed.

G. Reliability

Throughout the demonstration no reliability failures occurred. A total of 45 load/unload cycles were completed without failure.

H. Safety

\section{RF Emergency Stop}

An emergency stop using the on-board TTV emergency stop consists of shutting off all electrical control of the track and lid/latch solenoids, as well as unloading the hydraulics pump and cutting the fuel to the diesel engine. An emergency stop using the remote control station Hydraulics Off (emergency stop) button will shut off all electrical control of the track and lid/latch solenoids and unload the hydraulics pump while leaving the diesel engine running. 
The vehicle on board and remote control station emergency stop functions worked well when tested and used.

The supervisor's belt module RF emergency stop has three methods of executing an emergency stop on the TTV: by pressing the large red EMERGENCY STOP button on top of the portable transmitter, activating the tilt switch by tilting the transmitter more than 30 degrees from vertical, or by turning off the transmitter power. The first method activates the emergency stop relay, while the other two methods activate a transmitter-on relay. Both relays are reset by pressing the RESET switch on the front of the transmitter.

The supervisor's belt module RF emergency stop unit worked flawlessly until the final few days of the demonstration, when at first, it would occasionally perform an emergency stop that activated the transmitter-on relay. As the frequency of these occurrences increased, the transmitter-on relay was bypassed, so that only the emergency stop relay was used. This worked for 1 day, until the emergency stop relay behaved erratically, similar to the behavior of the transmitter-on relay the day before. On the final day of the demonstration, the transmitter was switched on to reset the emergency stop relay and promptly turned off to leave this relay in its reset state.

\section{Other Safety Features}

The motion siren and flashing engine active signals worked flawlessly throughout the demonstration.

7.2.4.4 Conclusion. Piloting the TTV was impaired by the lack of adequate horsepower and uneven power to the tracks. Nevertheless, these were both worked around through equipment modifications and creative piloting methods so that the TTV was successfully used for the demonstration. The telemetry test showed that the control system range easily met the 500-ft goal. The TTV on-board cameras provided adequate video signals to pilot the TTV. It was found that following several trips across the path, due to the narrow field of view an slow pan and tilt functions, it was easier to guide by the previous vehicle tracks and use the REMEX provided overview of the test site for piloting. The wireless video worked well and provided a clear video signal to the TTV operator. There was some interference from antenna mast. The RF emergency stop malfunctioned and repeatedly shut the TTV engine down, so one relay was bypassed to continue the demonstration. The ultrasonic sensors provided information to the TTV operator to dock at the targets. Although some crosstalk between the ultrasonic sensors gave erroneous readings, the readings were far enough from the actual readings so that the two could be

distinguished. The crosstalk between the ultrasonic sensors prevented completion of the test of the collision avoidance system. The TTV reliability was excellent, with no failures during the demonstration. Overall, the TTV control system worked well, with some difficulties. Fortunately, the difficulties were worked around through equipment modifications and creative operator methods.

\subsubsection{Maneuverability and Capability on Rugged Terrain, Precision and Accuracy}

The data quality objective was to document the maneuverability of the remote conveyance system used in the demonstration on rugged terrain by direct measurement. The test was to determine with a 95\% confidence level that for any specified target location, the average placement of the TTV was within 
1 radial foot of that target, and to document the level of variability in the terrain and the affect these variations had on vehicle maneuverability.

\subsubsection{Data and Data Reduction.}

A. Placement

The terrain encountered in Phase II testing was mostly soft sandy soil, with areas of rough broken up soil. Figure 29 shows the variability of the terrain. The topography of the test area consisted of two benches. The upper and lower benches were connected by a 10 degree sloped road. The TTV had a 300-ft long pathway that encompassed the upper and lower benches, as well as the 10 degree sloped road. Figure 12 shows the layout of the Phase II test site. The TTV docking station and targets were located at either end of the pathway.

Two methods were used to measure the TTV's position relative to the targets. The remote TTV operator used the onboard ultrasonic sensors to determine position to the targets. The other method consisted of manually measuring this distance using a measuring tape. A comparison of the data can be found in Appendix $\mathrm{H}$.

The ultrasonic sensors were mounted at the corners of the vehicle, with one pair facing forward and the other pair facing rearward. Each sensor is also pointed slightly down from horizontal, with various angles of pitch for each sensor (for no particular reason). The sensors are "pinged" in order: (1) forward left sensor, (2) forward right sensor, (3) rear right sensor, and (4) rear left sensor. The cycle repeats with the same sensor order.

\section{B. Maneuverability Course}

In addition to data gathered during the demonstration, performance testing was done to gather information about the TTV performance. A maneuverability test was conducted by setting up a track with markers, and driving the TTV through the path and timing the cycle. The path was $20 \mathrm{ft}$ wide, approximately $220 \mathrm{ft}$ long, in an oval pattern. The effect of operator proficiency was measured using the demonstration TTV operator for the first six cycles through the path and four other operators for the next four cycles. In addition, during the first six runs (with the experienced TTV operator), half of the cycles were done in direct track control, and half done in remote track control. All of the cycles with the novice operators were done in remote track control mode. Novice operators had not operated the TTV before this test. The measurements were taken by two people using a tape measure. They measured the distance form the centerline of the vehicle to the outside marker post on the course as the vehicle passed each marker post. The data in Appendix $\mathrm{H}$ show the distance by which the vehicle path varied from the intended path for each test.

C. Turning Radius and Ability to Climb 10 Degree Grade

Last, the vehicle turning radius was measured. The vehicle was turned about the smallest axis possible, and the radius was measured using a measuring tape. The performance of the vehicle while climbing the 10 degree grade was noted in the logbook during the demonstration. 


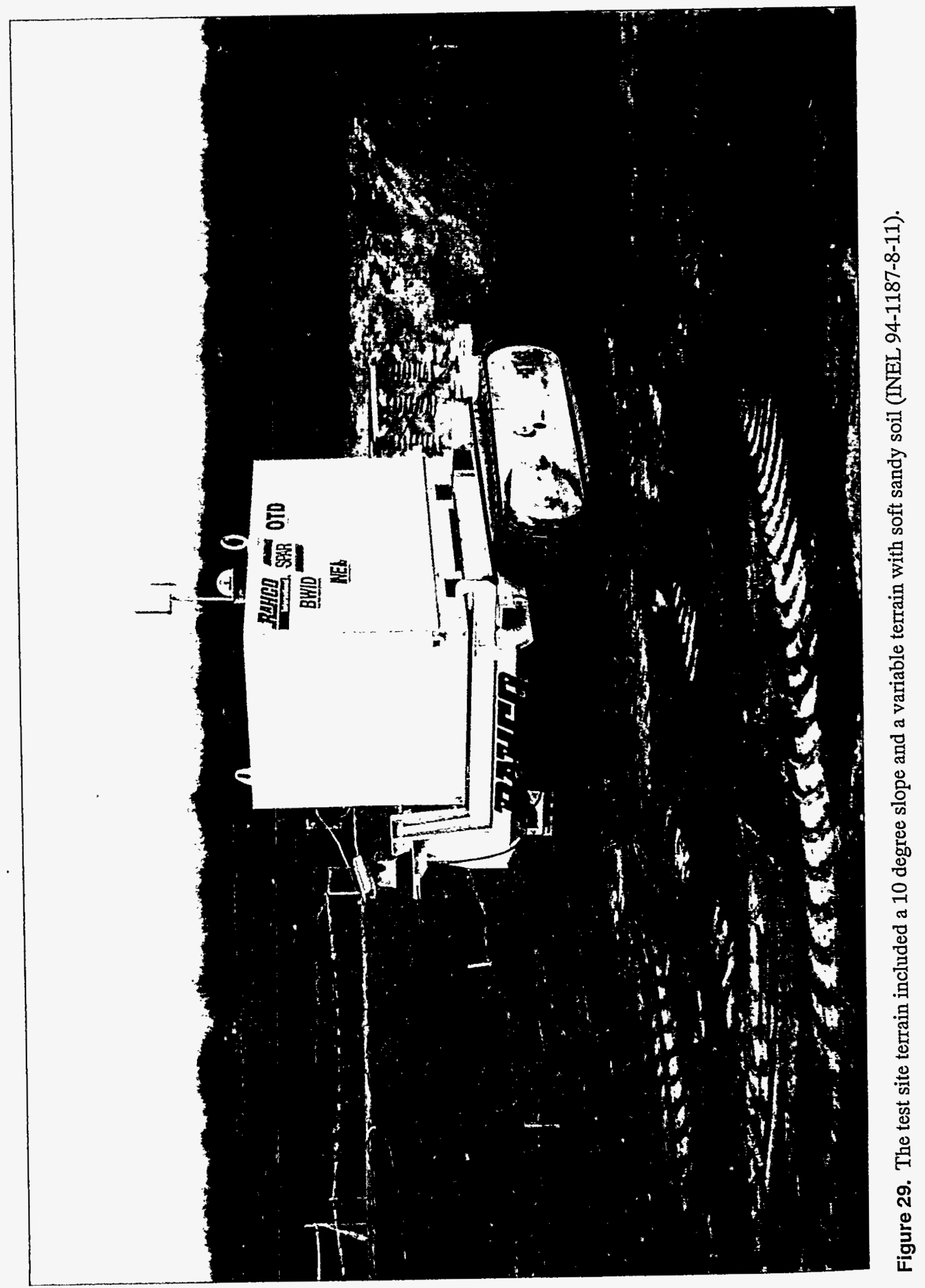



$\cdot$ 


\subsubsection{Data Analysis.}

A. Placement

There is a discrepancy between the measurements from the two methods used to measure position because the ultrasonic sensors were not accurate. This inaccuracy was caused by crosstalk between the sensors. A detailed description of sensor crosstalk is covered in the next paragraph. Due to this sensor problem, it was difficult for the operator to repeat the TTV's position to the targets. Consequently, $77 \%$ of the time the vehicle was placed within the 1-radial foot goal. Figure 30 shows a scatter gram of the TTV placement relative to the load station target. Note that the manual distance measurements taken from the load station were used in determining this percentage. The load station had two targets at right angles to each other, thus allowing two directions for measurement. These measurements were used to calculate the above accuracy. The first measurement taken during each test was used as the baseline measurement for that test, and all other measurements were compared to the first, or baseline, measurement.

At certain distances from the $4 \times 8$-ft plywood targets, the ultrasonic distance readings from one sensor often disagreed with the other sensor. The sensor with the questionable reading was always the one whose pinging sequence follows the one with the more reasonable (verified by manual measurement) reading. This occurred more often when the TTV was loaded with a container and an ITM. It suggested that the ping from the first sensor must have been reflecting back and forth between the large target and the container/ITM on the vehicle, and this. stray ping was being read by the second sensor before the ping from the second sensor (the genuine one) was received by the second sensor. To test this hypothesis, the cycle time was changed by doubling the time between successive pings. At this setting, similar discrepancies in the readings persisted, but they occurred at different distances from the target. Another problem was that sometimes the readings were smaller than the measured distances because the TTV was tilted so that a sensor was aimed at the diagonal braces on the plywood target.

Another way of determining if the TTV was perpendicular to the target was to line up the TTV until a pair of sensors reported similar distances. If interference among the pair of sensors occurred, the TTV could be moved backwards or forwards to avoid the crosstalk region, then the vehicle was rotated until the ultrasonic readings matched each other. The final TTV position was adjusted using the most reliable sensor.

B. Maneuverability Course

An average and standard deviation was calculated for each test run in the maneuverability performance test.

C. Turning Radius and Ability to Climb 10 Degree Grade

The turning radius data were averaged for the three cycles performed. No data analysis was required on the ability of the vehicle to climb the 10 degree grade. 


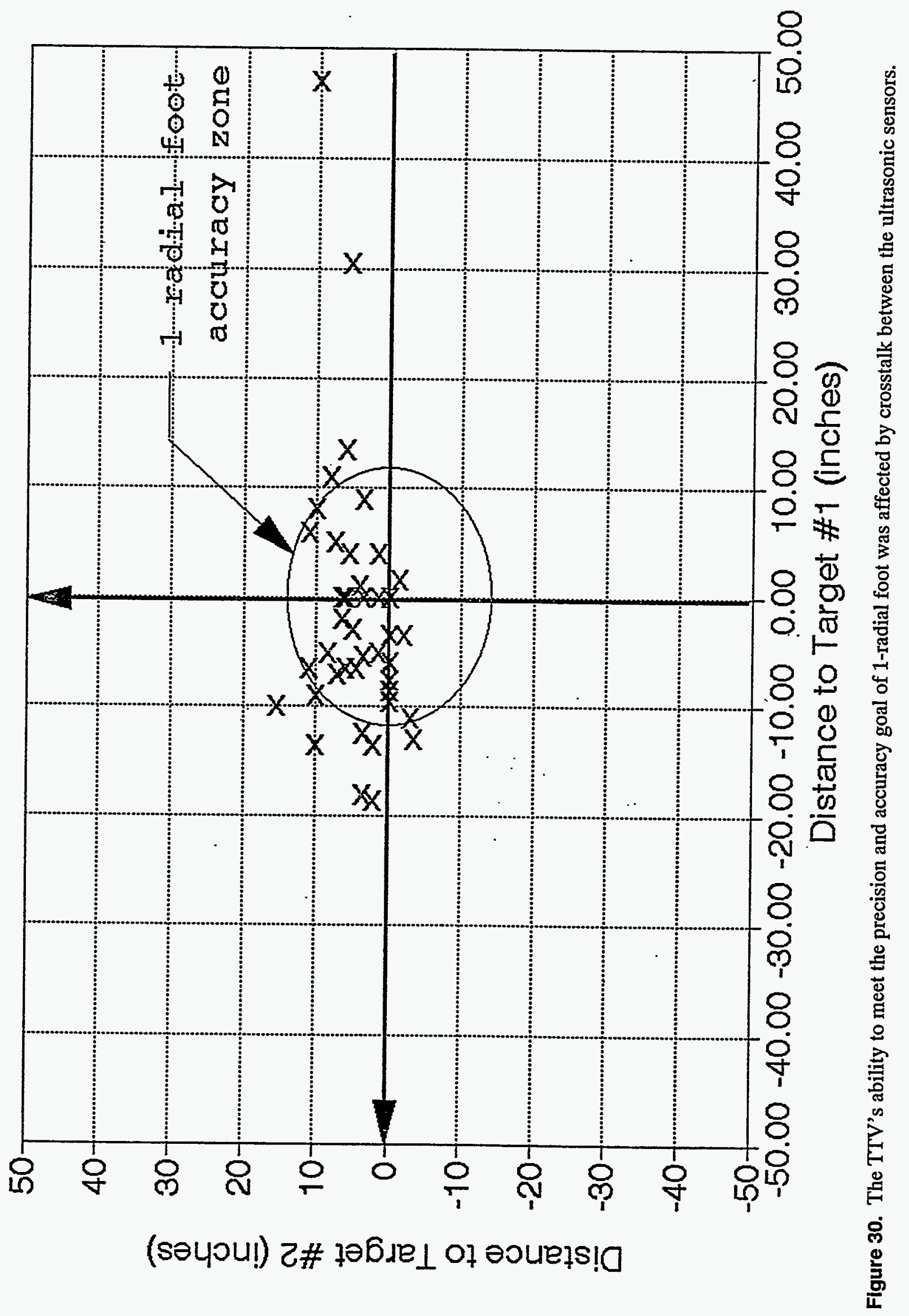




\subsubsection{Results.}

A. Placement

The performance of the TTV in the soft sandy soil terrain was excellent. It had good traction in the extremely soft soil at the demonstration site.

The vehicle was able to be positioned within the 1 radial foot goal $77 \%$ of the time. The inability of the vehicle to meet this goal $100 \%$ of the time was due to crosstalk between the ultrasonic sensors.

B. Maneuverability Course

The maneuverability performance test results showed that with an experienced operator, there was no significant difference between direct track and remote track control modes. However, operating in direct track mode required more skill and effort on the part of the operator. Even with an experienced operator, there was a learning curve. The first pass through the path the operator learned the path and was slightly further off of the desired path $(.7 \mathrm{ft})$, while on the remaining passes the operator maintained consistent distances from the desired path. The overall placement with the experienced operator was within $2.7 \mathrm{ft}$ of the desired path. Novice operators who had never operated the TTV, generally had more difficulty maneuvering through the path and were consistently further from the desired path than the experienced operator. On average, the novice operators were $4.9 \mathrm{ft}$ from the desired path. All ten of the cycles were completed in similar amounts of time. The lack of sufficient TTV horsepower affected the ability to navigate the TTV through the course because the TTV travel consisted of a series of polylines as stated in Section 7.2.4.3 A. See Appendix $H$ for additional details.

The vehicle maneuverability was also affected by the tracks of the vehicle. The original grousers had cleats that tended to dig into the soft soil and increase the friction load on the TTV. With the under powered engine, the affect of the grousers was magnified. Near the beginning of the demonstration, the cleats were covered with flat plates to improve vehicle maneuverability. The affect of the cleats on the maneuverability was significant. However, the maneuverability was still affected by the tracks of the vehicle throughout the demonstration.

During the testing, a problem occurred that necessitated deviation from the test plan. The video signal was consistently lost when the TTV was between markers 4 and 5 on the test course, leaving the operator with no data or visual information about the TTV. At these times, some cues for direction were provided to the operators by hand held radio until the camera views returned in order to prevent the vehicle from causing an unsafe situation. It is believed that the loss of camera signals was due to interference from the TTV antenna mast and other objects in the test area.

C. Turning Radius and Ability to Climb 10 Degree Grade

To determine its turning radius, the vehicle was turned about its axis. The goal was to turn 360 degrees on a 20 -ft wide path. As mentioned in Section 7.2.4.3 A, the vehicle did not turn to the right. The following data apply to the left hand turns that were executed. The vehicle was 
turned through 360 degrees three times during the performance test. The measured turning diameters were 21,22 , and $23 \mathrm{ft}$. This was primarily due to the insufficient horsepower of the vehicle and the uneven power to the tracks. Because of these two difficulties, the vehicle was not able to rotate about its center as designed, with one track moving forward and one track moving backward. Instead, the vehicle was skid steered by holding one track stationary and moving the other one forward, effectively rotating the vehicle about the end of the stationary track. With modifications to the horsepower and track control, the vehicle would easily meet the 20 - $\mathrm{ft}$ turning diameter goal and would actually turn about a $14 \mathrm{ft}$ diameter.

During each cycle of the demonstration, the vehicle climbed the 10 degree grade. With every cycle, the vehicle repeatedly climbed the grade without slipping.

7.2.5.4 Conclusion. Overall, the TTV did well in traversing the terrain encountered in Phase II testing. The TTV was positioned at the load station target within the 1-radial $\mathrm{ft}$ zone $77 \%$ of the time. The inability of the TTV to position within 1 radial $\mathrm{ft}$ every time was due to crosstalk between the ultrasonic sensors. Although the sensor crosstalk created problems, with minor adjustments as described in Section 11, the TTV placement could easily be improved to meet the 1-radial $\mathrm{ft}$ goal all of the time. During the maneuverability course test, it was found that while following a path, the operator learns the path on the first pass, and the ability to maneuver closely to the desired path is improved on subsequent passes. Operator proficiency plays an important role in vehicle maneuverability. In addition, the vehicle maneuverability would be greatly improved with adequate TTV power and equal track control. The vehicle did not quite meet the 20 -ft turning diameter goal because of the difficulties with inadequate horsepower and track control. Because of these factors, the vehicle was unable to turn by turning both tracks as designed but was skid steered using one stationary and one moving track. The vehicle was turned about an average of an $11 \mathrm{ft}$ radius, or a $22 \mathrm{ft}$ diameter. The vehicle had no difficulty climbing the 10 degree slope.

\subsubsection{Transuranic Environmental Evaluation}

The data quality objective was to determine the capability of the system to be used in a TRU-contaminated environment based on the following items: deployment, maintenance, repairability, reliability, and operating costs.

7.2.6.1 Data and Data Reduction. No data reduction was required. Qualitative data was gathered during the demonstration and throughout the design process.

7.2.6.2 Data Analysis. The data gathered and results presented represent the best knowledge and estimates of the demonstration team. However, a detailed analysis by an expert in TRU environment operations is suggested before design and/or deployment in a TRU environment.

7.2.6.3 Results. (The equipment was designed with operation in a TRU environment in mind, but due to prototype equipment and budget and schedule constraints, no attempt to industrialize the equipment was made. Additional design features would be required for evaluation for and deployment in a TRU environment. However, it would be feasible to design the equipment for operations in a TRU environment.) This section reviews the data gathered with regard to deployment, maintenance, repairability, reliability, and operating costs, and discusses several features that should be addressed for 
TRU environment operations. Additional suggestions for redesign for a TRU environment are provided in Section 11.

The ability of the equipment to be deployed remotely, the TTV to function on variable terrain, and the system integration are advantages in terms of deployment in a TRU environment. For deployment in a retrieval scenario, the remote excavator would need to be controlled by $R F$ link rather than a tether. (This control mode is an option offered by SPAR.) The maintenance, repairability, and reliability of the equipment in a TRU environment would need to be taken into consideration while designing for a TRU environment. From this test, it was determined that maintenance issues such as lubricating the joints on the REMEX and IEE would need to be addressed, either through a maintenance free design (such as self lubricating joints), or a design that takes maintenance in a bubble suit into consideration. The repairability of the equipment was not an issue during the demonstration because few repairs were needed, and repairs were not done wearing bubble suits as would be required in a TRU environment. Design for a TRU environment would need to include design for ease of repair while wearing bubble suits. In terms of reliability, this test, although short in duration, showed no reliability issues or problems with the equipment. Reliability studies would need to be considered in designing for a TRU environment.

7.2.6.4 Conclusion. The equipment used for Phases I and II testing was designed to test the concepts that were proposed in the original test plan and was not designed for a TRU environment. This equipment was successful in proving those concepts. Design of the equipment to operate in a TRU environment is feasible, and some possibilities for this design are discussed in Section 11. The cost to design and operate this system can be determined when specific requirements for operating in the TRU environment are defined.

\subsubsection{System Integration}

The data quality objective was to document the level of integration of the remote conveyance and IEE system and the affect that had on the smooth operation of the system.

7.2.7.1 Data and Data Reduction. No data reduction was required. Qualitative data were gathered during the demonstration and recorded in the logbook, on video tape, and in photographs.

7.2.7.2 Data Analysis. No data analysis was required. The results for system integration are presented in Section 7.2.7.3.

7.2.7.3 Results. There were three key factors involved in the successful integration of the remote conveyance and the IEE system: the remote conveyance system's ability to receive and deposit ITMs, the TTV placement system, and the integral design of the three systems-TTV, IEE, and REMEX.

The entire system consisting of the TTV, WTC, ITM, IEE, and REMEX worked extremely well together. Once the TTV was docked at the loading station, the TTV remote operator opened the WTC lid. Next, the remote excavator placed the loaded ITM into the WTC, detached from the full ITM, and then positioned itself for receiving an empty ITM. Figure 31 shows the IEE depositing a full ITM into the WTC on the TTV. The empty ITM was located on the TTV spring bed. The spring bed acted as an alignment tool for the IEE and was instrumental in helping the IEE mate to the empty ITM. Figure 32 shows the IEE retrieving an empty ITM from the TTV spring bed. With the help of the TTV placement system and the video system, the average cycle time for this operation was only 3.3 minutes. With some 


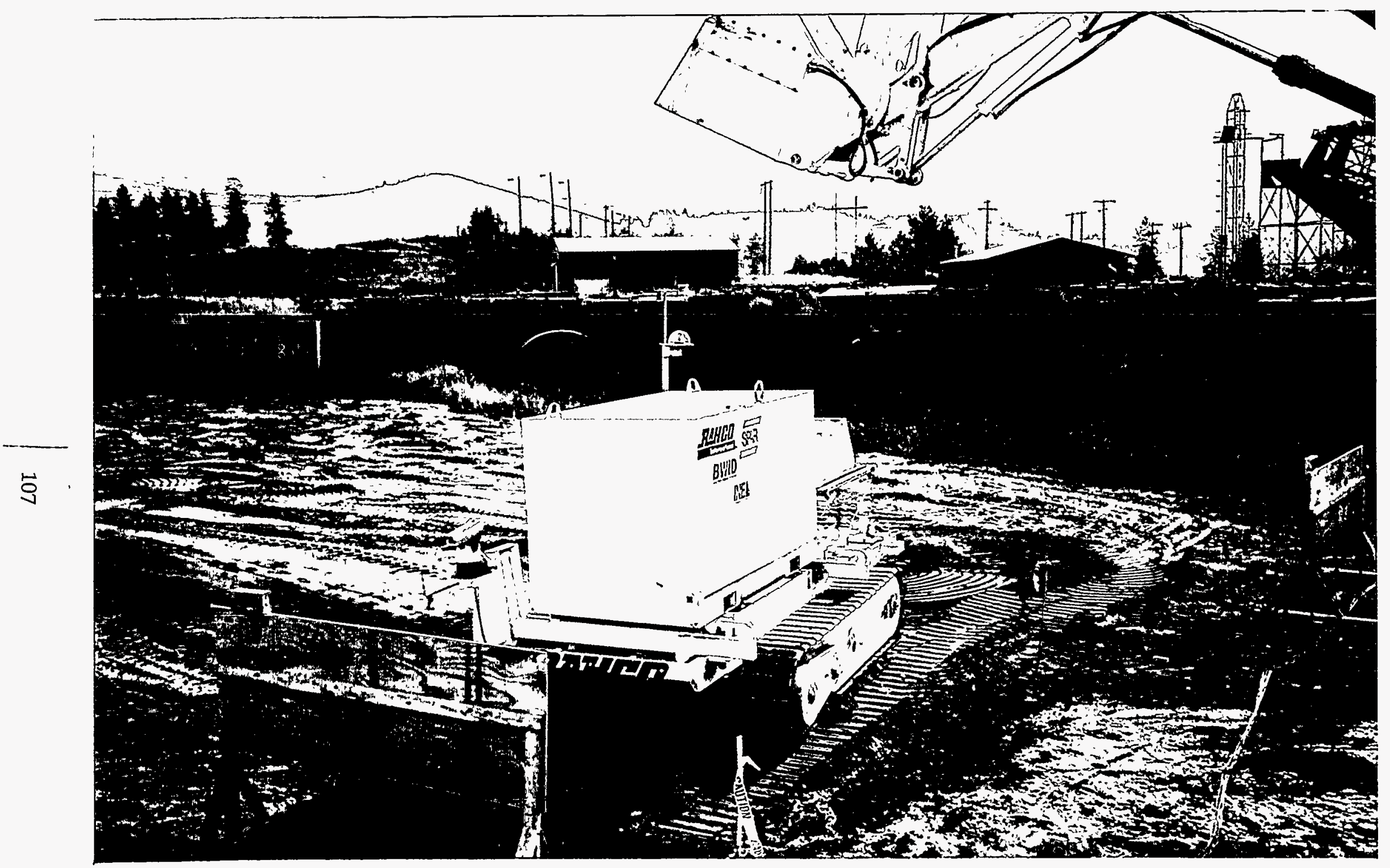

Figure 31. The capacity of the WTC allowed it to close over the ITM even when items in the ITM protruded above the top of the ITM (INEL 94-1187-1-10). 


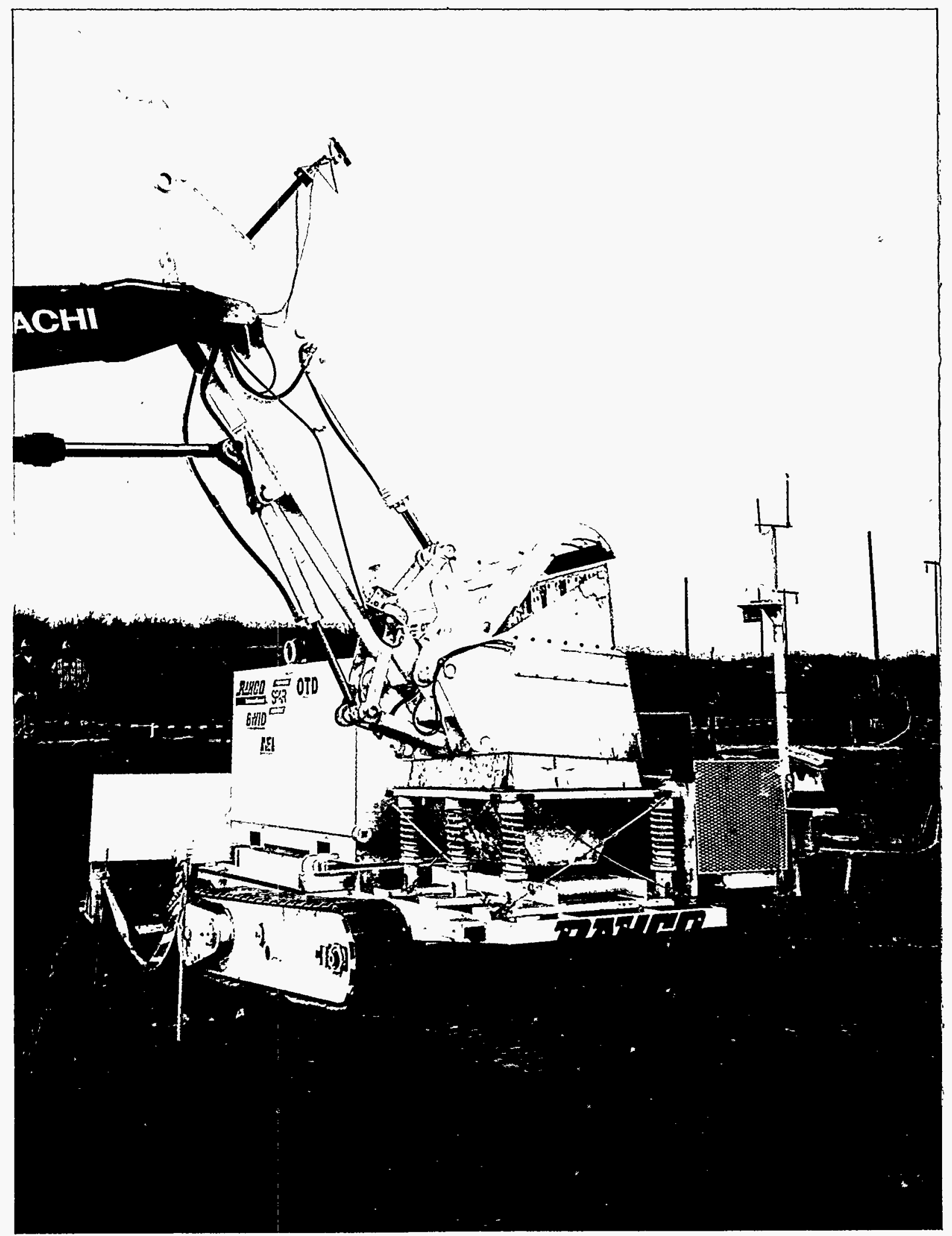

Figure 32. The spring bed on the TTV aided in attaching empty ITMs to the IEE because it provided flexibility in all three directions (INEL 94-1187-3-10). 
system improvements including a better vision system for the REMEX operator, this time could be easily reduced.

The TTV placement system consisted of targets at the docking stations, distance sensors (ultrasonic sensors), and video cameras. Figure 33 shows the TTV docked at the receiving station. Although the ultrasonic sensors were not as accurate as anticipated in the test plan, this did not negatively affect the integration of the system. The operator used the visual targets and the ultrasonic sensors to navigate the TTV to the target. Once the TTV was aligned with the target, the TTV operator and excavator coordinated with each other to accurately load and unload the ITM onto the TTV. Figure 28 shows the proximity of the remote operator control stations. Other video cameras strategically placed around the test site were helpful in the process.

The design of the IEE and TTV are complimentary to each other, which contributed significantly to the overall system performance. The flared skirting on the IEE and ITM were effective for mating the IEE to the ITM. The cradle design in the base of the WTC was essential for the integral placement of the ITM into the WTC. Also, the spring bed on the TTV proved to be an effective alignment device for the IEE to mate to the empty ITM.

7.2.7.4 Conclusion. The system proved to be highly integrated due to the systems approach of the design. The TTV and IEE mechanical systems were designed for full integration and worked well together. The spring bed on the TTV assisted with easy alignment and transfer of empty ITMs, while the tapered recess in the WTC assisted with alignment during depositing full ITMs. The target and video system worked well and was necessary for successful integration of the complete system. The systems approach used provided for smooth and easy integration between all three pieces of equipment, the TTV, IEE, and REMEX.

\subsubsection{Overall Phase II Demonstration Success}

The test plan states that Phase II could be deemed a success if sufficient data and operations experience were gained to assess the capability of the technology to accomplish its goals and if Environmental Restoration was provided with data to perform feasibility studies. The determination would be based on the following:

- Information regarding the ability of the entire system to effectively retrieve and transport waste is gathered and recorded for future use

- Sufficient data are gathered and recorded to evaluate the throughput of the system

- Sufficient data are gathered and recorded to evaluate the ability of the system to handle various waste forms and large objects

- Sufficient data are gathered and recorded to evaluate the maneuverability of the remote conveyance system on rugged terrain 


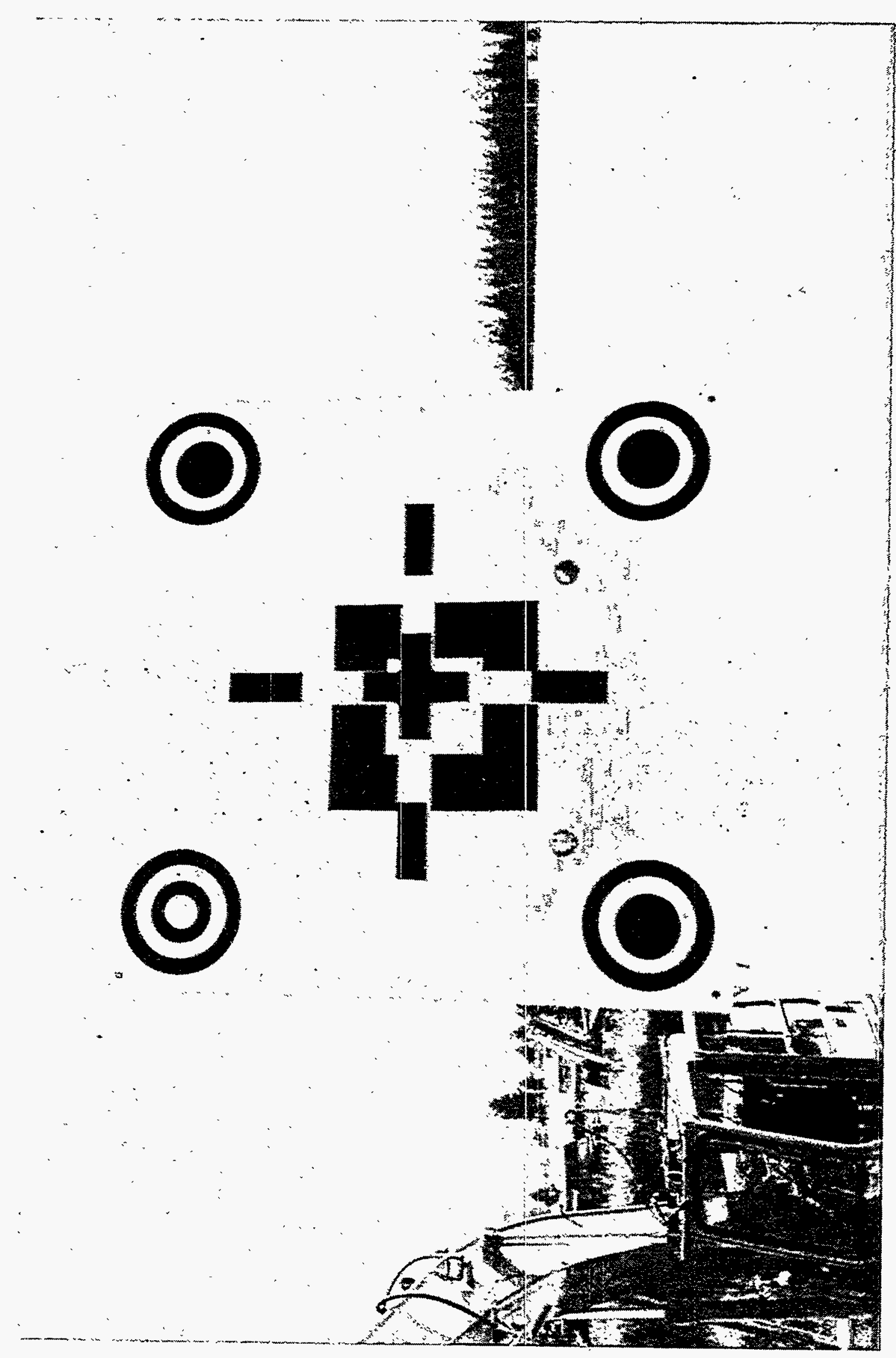

롴

:

5

矛

ت

这

焉宾

豙

व

号

蛋

홀

웜

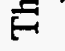

क

을 응

क

光

号

득 종

넝

뭉

응

S.

可

可

氙

을

정

롱

氙

용

点

0 년

E

लं

\%

ญ

혼 를 
- Information regarding the system integration between the remote conveyance system and the IEE is gathered and recorded for future use

- Information regarding the ability of the system to operate in a TRU-contaminated environment is gathered and recorded for future use.

7.2.8.1 Results. This report provides Environmental Restoration with technological data to perform feasibility studies. The ability of the system to effectively retrieve and transport waste was discussed in Section 7.2.4; the throughput achieved was provided in Section 7.2.1; the ability of the system to handle various waste forms was discussed in Section 7.2.2 and 7.2.3; data regarding the maneuverability were discussed in Section 7.2.5; the successful integration of the systems was reviewed in Section 7.2.7; and the ability of the system to operate in a TRU environment was discussed in Section 7.2.6.

7.2.8.2 Conclusion. The Phase II demonstration was extremely successful, not only in terms of data collection but also system performance. Information regarding the ability of the entire system to effectively retrieve and transport waste was gathered and recorded.

The systems throughput was recorded for each cycle and is documented in Appendix $H$. These data show that the conveyance system had a calculated production rate of $45.42 \mathrm{yd}^{3} /$ day during the demonstration. Although this throughput is less than anticipated, it can be shown that at optimum operating conditions, the system would easily reach 80 to $130 \mathrm{yd}^{3} /$ day.

Enough data were gathered to prove the system could sufficiently handle all of the various waste forms and large objects that were encountered during testing. The data were documented through various means; videotape, photographs, and logbook entries. Appendix $\mathrm{H}$ shows the raw data gathered and recorded about types and volumes of waste removed.

The data collected for the maneuverability tests showed that the TTV can repeat its placement to the targets within the specified 1 radial foot $77 \%$ of the time. The crosstalk between the ultrasonic sensors contributed to the target placement results. Appendix $\mathrm{H}$ presents the raw data for this test. The TTV travel over the terrain is well documented with both photographs and videotape. The test terrain was soft sand. The TTVs 300-ft pathway consisted of rough, broken-up terrain and a 10 degree sloped hill. The TTV had no problem negotiating this type of terrain or the 10 degree sloped hill.

System integration consisted of how well the conveyance system received and picked up the ITMs, how well the target system worked, and how well the IEE and TTV systems were integrated. Once again, the above subjects were well documented with photographs and videotape. The design of the system was excellent in regards to how well the IEE integrated with the TTV. The system performance was improved because of the design of this integrated system. In addition, it provides flexibility in the final application because the ITMs are versatile, flexible WTCs that can be adapted to work within many sorting and/or repackaging systems as well as creative excavation procedures. 


\section{OUTPUT/PRODUCTS}

\subsection{Theoretical Output of Process}

The output product of the IEE and telerobotic transport vehicle is an ITM containing untreated excavated buried waste. The ITMs would probably be shipped to a waste characterization and sorting facility where the waste could be sorted and repackaged and/or treated for final disposal. At the characterization and sorting facility, the ITMs would probably be dumped and sorted using robotic equipment or a glove box. If the ITMs need to be kept in interim storage while waiting for waste characterization and treatment, the ITMs could be made of less cost by material such as plastic. If a large number of ITMs are required during this process, it may be necessary to make the ITMs of less cost by material, possibly a disposable material. If the characterization and/or treatment facility is removed from the excavation building, a sealing lid would need to be made for the ITMs to prevent leakage during transport. If a bridge or gantry crane will be used (rather than a forklift) to move the ITMs within an interim storage area, a special sealing lid with crane hook attachments could be made.

\subsection{FY-94 Output}

\subsubsection{Data Output}

The data output of the project included this Technology Evaluation Report, Test Plan ${ }^{4}$, As-built drawings, photographs, videotapes, logbooks, and the project file (EG\&G Project \# WTD-CONVEY082-94 and WTD-BUCKET-083-94).

\subsubsection{Physical Output}

The physical results of the demonstration include the equipment, which is owned by and located at RAHCO, and the residual waste from the demonstration. The equipment includes the IEE and TTV. The REMEX that was used during the demonstration was on loan from SPAR Aerospace. The residual waste from the demonstration consists of the simulated waste used for the demonstration. This nonhazardous, nonradioactive waste was disposed of in accordance with local regulations at a landfill.

\subsubsection{Technology Transfer and CRADA Opportunities}

RAHCO International has applied for a patent for the IEE. RAHCO and SPAR are pursuing other waste management opportunities with other utilities, U.S. Government departments, other countries, and with private industry. Whenever possible, the project team is pursuing Corporation Research and Development Agreement (CRADA) opportunities to expand the use and applications for this technology. 


\section{PROBLEMS AND ISSUES FROM THE TESTING}

While testing of the REMEX/IEE and conveyance systems was completed successfully, occasional issues or problems arose during the tests that required schedule, equipment, or test plan changes. The effects of these problems and issues on the results of the tests were managed so that data objectives could be fulfilled. Several lessons were learned that will be useful for the BWID FY-95 integrated demonstration as well as future hazardous and radioactive waste retrieval and transport activities. This section discusses the issues and problems that occurred for the TTV, IEE, REMEX, control station, and demonstration layout. Specific recommendations to address these issues and problems are given in Section 11.

\subsection{Telerobotic Transport Vehicle}

The TTV was successful in meeting the objective of receiving waste from the remote excavator and transporting the waste from the digface with little dust generation. Following is a list of several issues regarding the TTV.

- The TTV maneuverability was impaired by several factors. First and foremost, the maneuverability was affected by the 47 horsepower engine in that it was insufficient power to turn a radius fluently. Because of the lack of power, the operator was not able to use the joystick for multidirectional movement but instead operated either forward/reverse or left/right separately. One solution that the operator used during the FY-94 tests was to move the vehicle forward and provide brief signals to turn while maintaining the forward momentum. Secondly, the tracks did not provide equal power for locomotion or turning. The left track provided less power than the right track. The cause of this problem is not known to date but is probably a combination of control system tuning, hydraulic imbalance, and mechanical resistance in the tracks. With this power imbalance, it would be expected that the vehicle would be able to turn to the right but perhaps not to the left. In fact, the opposite occurred; the vehicle was unable to turn to the right, but able to turn left. In considering the vehicle weight distribution, this can be explained as follows. For left turns, the left track has enough torque to move backwards, and the right track is able to move very slowly forward to keep from digging in. For right turns, the left track has enough torque to move forwards, but the right track cannot stay in motion due to the concentration of weight on the right side of the vehicle, so the TTV is unable to turn right. For straight line travel, the TTV tends to veer to the left when traveling forward and backwards due to the weight distribution and track power imbalance. It is expected that all of these problems may be unnoticeable if the vehicle horsepower were adequate. Third, the maneuverability was affected by the tracks on the vehicle. When turning in a stationary position, the tracks caused dirt to pile up and due to the insufficient engine power, the TTV was not able to traverse these piles. During testing, the cleats on the grousers were covered with flat plates that aided in maneuvering the TTV. This modification to the grousers improved the maneuverability significantly, but the vehicle was still impaired by the lack of necessary engine power. It is believed that if the appropriate engine power had been available, the effect of the tracks on the vehicle maneuverability would not have been an issue. Finally, it should be noted that the tests were performed in soft soil, which impacted the maneuverability of the TTV.

- The RF emergency stop on the supervisor's belt module malfunctioned repeatedly as discussed in Section 7. The supervisor's belt module RF Emergency Stop has three ways to stop the 
TTV. First, if the hand held RF Emergency Stop module is tilted beyond 15 degrees, it activates a relay to stop the TTV. Second, if the radio signal from the RF emergency stop to the TTV is lost, a relay on the TTV will open, cutting power to the hydraulics. Lastly, the module has a power switch that deactivates the radio link, and the vehicle senses no radio link and stops. It is not clear why the RF emergency stop malfunctioned during the test. In order to continue the test (the module repeatedly cut the power to the TTV during operations), the tilt power switch relay was first disconnected. In this mode, the RF emergency stop still functioned, with the exception of the tilt function. This mode eliminated the fail safe that cuts power to the TTV if the power to the module (battery) is lost, or if the module is turned off, but still allowed the module to shut the TTV down with the power switch button. The operators were made aware of this and extra caution was used. Operational procedures were reviewed with the RAHCO safety engineer to ensure safe operations. Later in the demonstration, the TTV received false signals from the Emergency Stop module, particularly when it was near the excavator or the remote control trailer. It is believed that these false signals were caused by radio interference.

- On one cycle, the thumb on the excavator was not aligned properly to clear the WTC due to the inadequate video views, and the thumb caught the WTC lid as it moved away after releasing the full ITM. The following day, after several cycles, the WTC lid would not close completely. At the time, it was not clear if the cause of this was the thumb contacting the lid on the previous day. It was speculated that it could have been caused by the lid opening arms not contacting the WTC simultaneously, so a bar was added to lock the two lid opening arms together. This bar inadvertently smashed the "lid closed" sensor by pulling the lid arms further in than they had previously been located. It is now believed that the stress induced on the WTC by the thumb caused a misalignment that led to metal fatigue, resulting in the WTC lid not closing fully.

- The ultrasonic sensors provided some erroneous readings due to sensor crosstalk. Fortunately, the data were so far from the actual distance that erroneous readings were easily detected. The sensor with the questionable reading was always the one whose pinging sequence followed the one with the more reasonable (verified by manual measurement) reading. This occurred much more often when the TTV was loaded with a container and an ITM. It suggests that the ping from the first sensor must have been reflecting back and forth between the large target and container/ITM on the vehicle, and this stray ping was being read by the second sensor before the ping from the second sensor (the genuine one) was received by the second sensor.

- The TTV operator's ability to clearly see what was going on was somewhat limited. The vision system was inadequate due to the limited field of view of the TTV cameras, the lack of an overview camera, and the slow pan and tilt features on the TTV cameras. Initially, to compensate for this, a line-of-sight person provided directions via radio to assist the TTV operator. It was soon found that the excavator cameras could be used to provide an overview for TTV operation and the line-of-sight person was no longer used. In order to compensate for the lack of overview cameras, the TTV operator and excavator operator worked in close coordination, using the excavator boom camera to get an overall view. The operators noted that more cameras including overview cameras would be helpful so that different views and angles could be seen. Lastly, the TTV station would benefit from a dual screen so both the overview and TTV cameras could be viewed simultaneously. Another problem was that when the TTV 
antenna mast was directly in line with the TV antenna and the remote control station, the video signal became snowy and/or flickered.

- Both the TTV and REMEX operator's vision was affected by the following factors. The fluorescent lights in the trailer created glare on the video screens, limiting visibility and increasing operator eye strain. Also, when the sun was at a low angle, the direct sunlight entering the video camera made it impossible to see through the cameras because the camera's auto iris function shut the lens to the point that no light entered.

- There were two issues that arose due to software problems on the TTV. When the TTV was in either direct or remote control modes and the camera pan and tilt functions were used, the TTV sometimes traveled when no travel signal was given. This problem was compensated for during the FY-94 demonstration by only operating the pan and tilt functions when the TTV was in lid/Iatch control mode, which disables the TTV propulsion. The second problem could have caused operator confusion about movement of the TTV. When the TTV forward camera was switched on, the TTV joystick responded to up motion with a forward movement of the TTV. However, when the TTV reverse camera was switched on, the TTV responded to an upward motion of the joystick by traveling backward. It was found that when operating the TTV remotely, the vehicle orientation (front or back) really did not affect the operation. In other words, from the remote operator's point of view, the vehicle does not have a "front," or a "back." It was determined that whatever camera was active was the "forward view" for the TTV, and it was desirable to always have the joystick respond to an upward motion with a forward motion from the remote operator's camera's point of view.

- Twice, when the pendant control module was connected or disconnected, a network failure of the on-board TTV control system occurred. After this type of malfunction, the system must be rebooted and several parameters re-entered. This malfunction does not cause any unsafe behavior.

- The latch that holds the WTC onto the TTV was only a two position latch, so that it either held the WTC onto the TTV or it held the WTC lid closed. When the TTV is traveling, it necessary to latch both the lid and the WTC to the TTV. For this demonstration, the latch was always left in the position where it held the WTC onto the TTV and the lid was not latched closed.

- The final issue was the throughput. The throughput was primarily affected by the TTV's limited horsepower, which reduced maneuverability and speed, and by the increased path length for the test. The limited vision system affected the throughput by limiting the speed of docking the TTV at the stations. The lack of color cameras affected the REMEX cycle times slightly.

\subsection{Innovative End Effector}

The IEE was also successful in meeting its objectives, including retrieving waste and transferring the waste to the TTV with little dust generation. Following is a list of several issues regarding the endeffector. Many of these issues were addressed in greater detail in Section 7.

- The ability to handle the various waste forms was affected by the teeth on the bucket and thumb, as well as the REMEX size. Some debris tended to get hung up on the end effector teeth, so during the demonstration, flat plates were added to cover the teeth on the bucket and 
the thumb. The REMEX is of an adequate size to handle the IEE when the excavator bucket is near the REMEX body. However, when the boom was fully extended with a full ITM, the IEE was too heavy for the REMEX, causing the REMEX to tilt. This affected the size and weight of waste forms that it could handle.

- During the first part of testing, the IEE affected the throughput that was achievable. The end effector had an overflow slot so that excess waste could be directed through the slot, limiting the volume of waste in the ITM. Unfortunately, the overflow slot was too big, causing most of the waste to be directed out of the ITM. During the initial stages of testing, a plate to partially cover the overflow slot was designed, built, and put over the slot. The plate was tapered to assist in directing the waste into the ITM. This plate corrected the problem and following its implementation the IEE was able to fill the ITM completely.

- For in-cab operations, the IEE interferes with the operator's view of the digface. During the demonstration, the operator compensated for this by viewing and arranging the digface with the IEE without an ITM attached, then returned with the IEE and ITM to remove a scoop of waste. It was suggested than an in-cab camera view of the digface would aid in operations should incab operations be required.

\subsection{REMEX}

The REMEX was successful in retrieving the waste. Following is a list of several issues regarding the REMEX.

- The REMEX operator's vision was somewhat limited. First, the dark lines on the WTC that were used to aid in ITM placement were not sufficient aid because the visibility in the video displays was limited. In addition, when the sun was at a low angle, the shadows from the REMEX boom shaded the waste pile and affected ability to see the waste and the digging operation. The REMEX operator noted that more cameras would be helpful so different views and angles could be seen. The operator also expressed that the vision system needed higher resolution cameras to see the detail on the digface and reduce operator fatigue. The operator indicated that using color cameras would provide additional cues to depth perception.

- The REMEX sensor that detects bucket curl position was bolted to the bucket pin. The pin was missing a grease fitting, and the lack of lubrication caused a torque to develop that was transferred to the sensor bolts. As a short term fix two of the bolts were replaced, and the bucket linkage was welded to the pin so that the weld would hold the tension rather than transferring it to the bolts. Following the Phase I test, this temporary solution was replaced with a permanent solution where the four bolts were all replaced, a grease fitting was added to the pin, and the pin was greased.

- The REMEX track speeds were unequal; the left track was very weak. This affected the turning because it over swung. The drive system on the REMEX left track is old and worn out and should be replaced for future use.

- The REMEX camera system was not designed for outdoor use. Because no inclement weather occurred during the demonstration, this did not cause difficulties during the demonstration. The 
cameras were only covered for weather protection during one night. However, the inability of this system to withstand outdoor use could have presented significant difficulties. The camera system should be a rugged, weather-ready system.

- There were repeated fuse failures. At one point during the tests, the REMEX functions were redistributed to different power busses. Fuses still failed. A short in the sensor on the bucket that shows the ITM attach/not attach was located. The wire was cut, which disabled the sensor in order to continue the tests. This was done towards the end of the Phase II demonstration.

- The drums full of Portland cement were filled with dry cement powder rather than wet cement (sludge) because of a miscommunication. Although the dry cement was not planned, it proved valuable in understanding the mechanisms that might be involved in buried waste retrieval. The 55-gal drums full of dry cement were easily bent and became deformed by the end effector as they were moved around the digface in preparation for removal. The dents in the drums pressurized the contents so that when the drum was later punctured by the end effector, the contents sprayed out in a $30-\mathrm{ft}$ plume of dry cement. This possible method of contamination spread might otherwise not have been considered during buried waste retrieval. 


\section{FULL-SCALE IMPLEMENTATION}

\subsection{Overview}

The TTV and REMEX are systems that would be useful for both retrieval and transport of radioactive and hazardous materials. Because the system is remotely operated, it provides protection for personnel. Considerations for full-scale implementation are discussed in this section. The section is divided into implementability, effectiveness, and cost to aid Environmental Restoration in comparing this technology to others during remedial investigations. These sections are based on the actual equipment demonstrated in FY-94 and that if the suggested modifications are implemented, the throughput rate of the systems would improve.

\subsection{Implementability}

Implementability includes the following:

- Ability to construct and operate the technology

- Reliability of the technology

- Ease of undertaking additional remedial actions, if necessary

- Ability to monitor effectiveness of remedy

- Ability to obtain approvals from other agencies

- Coordination with other agencies

- Availability of offsite treatment, storage, and disposal services and capacity

- Availability of necessary equipment and specialists

- Availability of prospective technologies.

\subsubsection{Ability to Construct and Operate the Technology}

As discussed throughout this report, the systems were successfully designed, built, and tested during FY-94. Section 7 discusses to operator's ability to operate the equipment. For the purposes of comparing these technologies to others, the following should be noted. Both the IEE and conveyance system were custom assemblies. The excavator was an off-the-shelf item that was modified for remote control and to add the joystick control arm for in-cab operations. Now that the designs have been completed, the lead time for the IEE is estimated to be 3 months, and the lead time for a duplicate conveyance system is estimated to be 4 months. The uncertainty on these lead times would stem from multiple orders and is estimated to be \pm 1 month. The modifications recommended for the equipment (see Section 11) would affect the lead times as further engineering is required. It is estimated that the initial order for the modified equipment will take 7 months. 
Another issue that may affect the ability to operate any technology is power. The remote control station air conditioning power could be provided by a generator or by line power and therefore is versatile enough that it should not adversely affect the ability of the technology to operate at various sites.

\subsubsection{Reliability of the Technology}

Reliability as discussed here focuses on the likelihood that technical problems associated with implementation will lead to schedule delays. During the tests conducted, no significant failures occurred other than what would be expected with prototype equipment, making this section an unknown. However, several technical factors should be addressed during set up of the equipment to ensure no schedule delays. These factors include ensuring proper power, routing wiring so that no interference from other electrical cords occurs, ensuring no RF interference, ensuring wires for remote excavator controls are routed or replaced with RF links, and planning paths so that the wiring is not in the path of the remote excavator and conveyance systems.

Recurring fuse failures on the REMEX led to two causes. First, it was found that signal load on one bus was too high, causing a power overload. During the FY-94 demonstration, this problem was corrected by routing some signals to alternate busses. This problem will need to be addressed in future iterations to improve reliability. Secondly, a short circuit on an ITM attach sensor was found. This problem was also repaired in the field during the FY-94 demonstration. It is recommended that the ITM attach prototype sensors be replaced with more rugged sensors for future iterations of the IEE.

Factors that affected the reliability of the systems in FY-94 included having a highly compressed schedule and tight budget constraints; prototype hardware packaging and installation; and using low cost, prototype sensors. The sensors and prototype hardware packaging and installation could be engineered to improve reliability. There is no technical reason why reliability could not or would not be equal to or significantly better than commercially available industrial heavy equipment in a production design.

Scheduled maintenance included lubricating the excavator and refueling both the excavator and conveyance systems. Scheduled maintenance required approximately $1 / 2$ hour/day.

\subsubsection{Ease of Undertaking Additional Remedial Actions}

The technologies discussed in this report allow the waste to be retrieved and transported from the digface. They do not in any way affect the future treatment of the waste. However, waste assay may not be possible while the waste is contained in an ITM. The only technology that may be precluded by use of these systems is in situ treatment. If other technologies are used in conjunction with these (such as spraying the digface with fixants or surfactants to further limit airborne dust), these additional technologies may affect the characterization or treatment technologies selected.

Removal of the buried waste and placement into ITMs (small $1.4 \mathrm{yd}^{3}$ packages) should aid in subsequent sorting and repackaging activities. In addition, the ITM is a versatile, easy to handle container that is well suited to interim storage or attaching a lid for transport. The ITM could be made of a lower cost or disposable or recyclable material if required to limit decontamination processes. Another option would be to transfer the waste to another container so the ITMs could be reused without decontamination each cycle. 


\subsubsection{Ability to Monitor Effectiveness of Remedy}

The effectiveness of the remedy can be monitored several ways. First, the IEE must remove the waste. The effectiveness of this can be measured several ways. First of all, the video images can show operators where they have dug and allow them to see if waste is visible. The second method would be to use characterization technologies (such as BWID Digface Characterization) to determine if additional waste remains. The end effector must also minimize potential airborne contamination spread. This can be monitored by using air monitors such as those described in this report and used in the tests or by continuous air radiation monitors for a hot retrieval. The waste conveyance system should receive the waste with minimal or no dust spread and convey the waste from the digface with no loss of waste. The effectiveness of the TTV to receive the waste with little or no dust generation can again be monitored using air monitors. The waste conveyance system is fully contained and will not lose any of the waste once the lid is closed.

\subsubsection{Ability to Obtain Approvals from Other Agencies}

The permits and approvals required from other agencies for full-scale operation would depend on the site to be remediated. There would not be any special permits required for the excavator/end effector or the conveyance vehicle. The NEPA documentation should address the fumes produced by the vehicles.

\subsubsection{Coordination with Other Agencies}

Coordination with other agencies would depend on the site to be remediated. For example, at the INEL, Environmental Restoration would need to coordinate with DOE, the State of Idaho, and Environmental Protection Agency (EPA) Region 10. Other sites would need to coordinate with agencies listed in their agreements with the State(s). The technologies discussed in this report would not require additional coordination with other agencies.

\subsubsection{Availability of Offsite Treatment, Storage, and Disposal Services and Capacity}

Both the excavator/end effector and conveyance systems would not preclude any of the available offsite treatments, storage facilities, or disposal services from being used. The waste being retrieved for a specific site would dictate which of these services could be used. Once that was determined, the availability of those services could be addressed.

\subsubsection{Availability of Necessary Equipment and Specialists}

The availability of the necessary equipment is discussed in Section 10.2.1. During FY-94, the operators who were used had been previously trained for heavy equipment operation. However, with the Remote Control Station and remote excavator joystick control arm, previous experience with heavy equipment was not a requirement for the operators. For full-scale implementation in a radioactive environment, we suggest the operators have training for responding to incidents and emergencies based on the configuration of the buried waste, have experience with excavating waste piles, and have training on the remote equipment in a nonhazardous, nonradioactive environment. A risk analysis should be performed to determine the training requirements for each specific site and equipment combination. 


\subsubsection{Availability of Prospective Technologies}

The systems were demonstrated in September 1994. This equipment is currently being modified as

discussed in this report. A full-scale BWID of the upgraded equipment is currently scheduled for the summer of 1995.

The BWID Program teamed with RAHCO and SPAR to develop the remote conveyance system and IEE demonstrated in FY-94. SPAR provided the TTV control system and REMEX. RAHCO designed and built the IEE and TTV mechanical systems. The REMEX was not part of the originally requested scope of work but was added in the proposal as a value-added item. The REMEX was developed before FY-94 by SPAR.

\subsection{Effectiveness}

Effectiveness entails (a) overall protection of human health and the environment, (b) compliance with applicable or relevant and appropriate requirements (ARARs), (c) long-term effectiveness and permanence, (d) reduction of toxicity, mobility, and volume through treatment, and (e) short-term effectiveness. Compliance with ARARs is site specific and is, therefore, omitted from this discussion. The other areas of effectiveness are discussed below.

- Overall Protection of Human Health and the Environment

- How the technology provides human health and environmental protection.

- Long-Term Effectiveness

- Magnitude of residual risk and

- Adequacy and reliability of controls.

- Reduction of Toxicity, Mobility, and Volume

- Treatment process used and materials treated

- Amount of hazardous materials destroyed or treated

- Degree of expected reductions in toxicity, mobility, and volume

- Degree to which treatment is irreversible

- Type and quantity of residuals remaining after treatment.

- Short-Term Effectiveness

- Protection of community during remedial actions

- Protection of workers during remedial actions

- Environmental impacts

- Time until remedial action objectives are achieved.

\subsubsection{Overall Protection of Human Health and the Environment}

10.3.1.1 How the Technology Provides Human Health and Environmental Protection. These systems are an improvement over conventional excavators and waste transport vehicles for two reasons. First, the equipment is remote, reducing the risk to workers. Second, the equipment works in 
concert to control dust, controlling the amount of airborne contamination spread. Therefore, these systems protect both human health and the environment.

\subsubsection{Long-Term Effectiveness}

10.3.2.1 Magnitude of Residual Risk. Magnitude of residual risk refers to the residual risk remaining from untreated waste or treatment residuals at the conclusion of remedial activities. The risk would be a function of the waste being retrieved and the treatment being used and cannot be addressed at this time. However, the retrieval/transport system would affect whether or not all the waste was removed from the site. Because of this, it is recommended that a sizing method be available onsite to reduce the waste into a size that the end effector and waste conveyance system can handle.

10.3.2.2 Adequacy and Reliability of Controls. The adequacy and reliability of controls refers to the management of treatment residuals or untreated wastes that remain at the site. Again, the treatment residuals would depend on the specific waste and treatment method chosen. However, the retrieval/transport system would affect whether or not all the waste was removed from the site. Because of this, it is recommended that a sizing method be available onsite to reduce the waste into a size that the end effector and waste conveyance system can handle. Alternatives to the sizing method would be to assess containment systems and institutional controls to ensure that any exposure to human and environmental receptors is within protective levels.

\subsubsection{Reduction of Toxicity, Mobility, and Volume}

10.3.3.1 Treatment Process Used and Materials Treated. The IEE and conveyance vehicle do not treat the waste so this section is not applicable. However, it is important to note the types of wastes the systems can retrieve and transport and any limitations of the systems. As previously discussed, there were a few design modifications done to the IEE to improve its efficiency. The first modification involved reducing the overflow opening on the IEE. Prior to this modification, the waste tended to fall through the opening creating dust and only capturing $25 \%$ of the waste being excavated. The second modification involved replacing the end effector ripper teeth with a grader bar and also adding a plate to the tip of the thumb. The teeth and the thumb breached drums and also caused debris to hang up on the end effector. Once these modifications were completed, the IEE became efficient in handling all of the waste forms. The test data shows that the rate of removal more than doubled after these modifications were completed. The conveyance system is only limited in the size of materials it can handle. The TTV can carry $4 \times 4 \times 8$-ft boxes and/or large objects in the WTC without an ITM. The systems should be able to retrieve any hazardous of radioactive materials including sludges, slurries, dirts, and debris.

10.3.3.2 Amount of Hazardous Materials Destroyed or Treated. The IEE and conveyance vehicle do not treat the waste so this section is not applicable to this technology.

10.3.3.3 Degree of Expected Reductions in Toxicity, Mobility, and Volume. The IEE/excavator and waste conveyance system do not change the waste toxicity or volume. The systems do address the mobility of the waste in a positive way in that they retrieve the waste and transport it from the contaminated area for subsequent storage or treatment. In a negative sense, the small fraction of waste that becomes airborne may have an increased mobility. 
10.3.3.4 Degree to Which Treatment is Irreversible. The IEE and conveyance vehicle do not treat the waste so this section is not applicable. However, it is important to note that the end effector and conveyance system actions are totally reversible in that the waste could be put back into the pit if desired.

10.3.3.5 Type and Quantity of Residuals Remaining after Treatment. The IEE and conveyance vehicle do not treat the waste so this section is not applicable. However, it is important to note the secondary wastes that would be produced as a result of using the systems. The types of wastes include

- Hydraulic fluid

- Diesel fuel

- Bearing grease

- Residuals from decontamination process

- Gloves

- Bubble suits

- Rags

- Air filters (oil quenched)

- Fuel filters

- Exhausts (proportional to engine speed).

The volume of the contaminated material is dependent on the number of hours that the equipment is operated.

\subsubsection{Short-Term Effectiveness}

10.3.4.1 Protection of Community During Remedial Actions. This aspect of short-term effectiveness addresses any risk that results from implementation of the technologies. For these systems, the following risks should be considered: airborne dust generated from the excavator at the digface during transfer to the conveyance system and from driving both vehicles; and risks associated with transporting the wastes. As discussed in Section 7, the systems work together to control the spread of airborne contamination by eliminating the dust during the transfer. Dust was still generated at the digface but lessened as the operator gained experience using the equipment. This dust could be further reduced through the use of contamination control at the digface. The vehicles would drive in the area and could potentially spread contamination from that activity. Finally, the waste conveyance system would transport the waste from the digface to some area for unloading. The transportation distance and path should be assessed to ensure that the community is protected at all times. If transporting the conveyance system out of the containment building, the ITMs would need lids with seals or the WTCs would need seals and a lid latch mechanism. All transport vehicles would need to be decontaminated before exiting the building. 
10.3.4.2 Protection of Workers During Remedial Actions. These technologies are remote and, therefore, protect the workers from the hazards and radiation at the digface. The only time that workers may be required to enter the area is for scheduled and unscheduled maintenance or for unplanned spill cleanup.

10.3.4.3 Environmental Impacts. Environmental impacts refer to the adverse impacts that occur during the construction and implementation of a technology. These impacts would be dependent upon the specific site being remediated and administrative controls over the area. Both remote systems would not adversely effect the environment. Both systems would generate noise and exhaust fumes and would require a path. These hazards are considered minimal and standard in the construction environment.

10.3.4.4 Time Until Remedial Action Objectives are Achieved. The results in Section 7 indicate that with the existing power limited, prototype system, approximately $45.4 \mathrm{yd}^{3} /$ day of waste was retrieved and transported using these systems. With the modifications proposed in Section 11, the system will be able to retrieve far greater than the BWID objective of $80 \mathrm{yd}^{3} /$ day.

\subsection{Cost}

Cost includes the following:

- $\quad$ Capital costs

- Operating and maintenance costs.

The systems discussed above are remote and are estimated to have a useful life of 10,000 hours. Therefore, these systems could protect personnel from radiation for that period of time (maintenance time is not considered in operating hours). These systems replace a minimum of two operators. DOE-established dose limits are limited even more by the contractor. The current administrative control during routine conditions is $1 / 5 \mathrm{rem} / \mathrm{year}$, and it is recommended that workers not be exposed to more than $300 \mathrm{mrem} / \mathrm{week}$. It is impossible to draw concise conclusions regarding the amount of exposure reduction without knowing the exact site conditions.

For example, if operators are exposed to 100 mrem/8-hour shift, and assuming two operators at a time, the remote equipment over its useful life would reduce exposure by $250 \mathrm{rem}$. The following sections provide a discussion regarding the costs for these remote systems. The total capital costs for production versions of both systems are estimated to be between $\$ 1,000,000$ and $\$ 2,000,000$. Based on the above assumptions and this particular example, the cost efficiency of these systems is between $\$ 4,000$ and $\$ 8,000 /$ person-rem.

\subsubsection{Capital Costs}

Capital costs consist of direct (construction) and indirect (nonconstruction and overhead) costs. Direct costs include construction costs (materials, labor, and equipment to prepare for use), equipment costs, land and site development costs, buildings and services costs, relocation expenses, and disposal costs. Indirect capital costs include engineering expenses, license or permit costs, startup and shakedown 
costs, and contingency allowances. Table 8 depicts the estimated capital equipment costs associated with the IEE/excavator and the conveyance system. Because of unknown and only rough order of magnitude estimates, only the equipment costs are included.

Land and site development costs may be required for operating the conveyance system and for pavement for the conveyance to prevent or limit dust generation/contamination spread. The buildings and services refer to costs that are additional costs that may be required as a result of using these technologies. This is difficult to estimate because the site conditions are unknown and so are the other technologies that are required. The building, if required, would need to be big enough to house the equipment and effectively maneuver within it and may require seals and filters for contamination control. In addition, some type of air lock may be required for the conveyance system. Both systems are diesel powered and the fumes may need to be dealt with if personnel needed to enter the building. Relocation expenses refer to costs for temporary or permanent accommodations for affected nearby residents. This cost would depend on the site that was being remediated and would not be affected by the equipment itself. Disposal costs include the costs for transporting and disposing of the waste material. This cost is also site specific and therefore cannot be estimated at this time. However, the only added waste as a result of these systems that would need to be treated/disposed would be the waste generated from decontaminating the equipment and from maintenance of the equipment (used fluid, oil, broken parts, etc.).

Indirect capital costs for engineering expenses would include demonstration support, program management, performance of the tests, quality assurance, safety, and shipping expenses. Licenses and permits would be obtained based on the particular site being remediated. These costs would not be affected by the remote systems. Startup and shakedown costs are the estimated costs to ensure the remote systems are operational and functional. Contingency refers to money to cover costs resulting from unforeseen circumstances such as adverse weather, waste variability not anticipated, equipment repair, explosion, and fire.

\subsubsection{Operating and Maintenance Costs}

Operating and maintenance costs are incurred after construction costs and are necessary to ensure the continued effectiveness of a remedial action. The following are considered operating and maintenance

Table 8. Estimated capital equipment costs. Costs are subject to change and depend on the level of complexity of requirements for each machine and each specific site to be remediated.

\begin{tabular}{cc}
\hline \multicolumn{1}{c}{ Type of cost } & Estimated cost \\
\hline Equipment costs/excavator: & \\
IEE & $\$ 35,000-\$ 50,000$ \\
ITM & $\$ 2,500-\$ 3,500$ \\
Control system & $\$ 230,000-\$ 300,000$ \\
Excavator & $\$ 300,000-\$ 500,000$ \\
Equipment costs/conveyance: & \\
SGV & $\$ 300,000-\$ 400,000$ \\
WTC & $\$ 10,000-\$ 15,000$ \\
Control system & $\$ 300,000-\$ 350,000$ \\
\hline
\end{tabular}


costs: operating labor, maintenance materials and labor, auxiliary materials and energy, disposal of residues, purchased services, administrator, insurance, taxes, licensing, maintenance reserve and contingency funds, rehabilitation, and periodic site review.

The expected useful life as discussed earlier is approximately 10,000 operating hours for both systems. The operation costs for the life cycle of the technologies are difficult to estimate because their use would ultimately be affected by the site they are being used at and the equipment with which they are being deployed. 


\section{CONCLUSIONS AND RECOMMENDATIONS}

Successful demonstration and testing of the remote conveyance system, IEE, and REMEX was performed in September 1994. This testing was the first testing of the integrated systems and showed the status of the technology and the capabilities of the systems. Test conclusions and recommendations for future development of the systems follow.

\subsection{Conclusions}

A remote conveyance system and a remote excavator equipped with a detachable end effector were successfully demonstrated and tested. The remote systems accomplished remote retrieval, transfer, and transport of simulated buried waste while limiting dust generation at the dumping/transfer location. The prototype systems showed that waste could be successfully handled remotely and in fact, controlled the amount of potential airborne contamination spread. Furthermore, because the systems are remote, personnel that would otherwise be in the hazardous or radioactive area are protected from these hazards. In addition, there is no waste generated as a result of manual operations, such as bubble suits and gloves, that would require subsequent treatment and disposal. When the recommended modifications (see Section 11.2) to the systems are made, the remote systems will improve the retrieval rate to approximately $130 \mathrm{yd}^{3} /$ day. These systems (once modified for use in a TRU environment) will be able to be used by DOE Environmental Restoration to remediate contaminated buried waste sites across the DOE complex.

\subsection{Recommendations}

Recommendations regarding the IEE, REMEX, conveyance system, vision systems, and controls/displays are given below. These recommendations are based on the issues and problems encountered during testing as well as engineering judgment.

\subsubsection{Innovative End Effector}

- A short occurred in the ITM attach sensor during the FY-94 demonstration. The ITM prototype sensors should be replaced with more rugged sensors.

- Although the following did not occur during the demonstration, it is suggested that these issues be addressed before deployment in an actual retrieval. A method should be developed for handling an overfull ITM bucket in case the WTC will not close on an overfull ITM. If the ITM drops debris into the WTC and fills up its loading slot/position, a method should be in place to clear the ITM slot.

\subsubsection{Conveyance System}

- A more powerful TTV engine would improve maneuverability and increase speed of the TTV. The diesel engine used in FY-94 was 47 horsepower; it is recommended that an engine of 100 or more horsepower be used on this vehicle for future use.

- The TTV tracks did not provide equal power for locomotion or turning. The cause of this problem is not known. It is recommended that the cause be identified and corrected so that the tracks perform equally. 
- Due to a software problem, when the TTV was in either direct or remote control modes and the camera pan and tilt functions were used, the TTV sometimes traveled when no travel signal was given. This software problem will need to be corrected.

- The crosstalk between the ultrasonic sensors should be addressed so that accurate readings and obstacle avoidance can be achieved.

- In order to improve vehicle maneuverability, the cleats on grousers were covered with flat plates. Future grousers could use a rubber track to further improve maneuverability.

- The RF Emergency Stop malfunctioned repeatedly, as discussed in Section 7. This system would need to be improved for future operations.

- The TV antenna should be moved to the top of mast for the TV signal because when the antenna is between the mast and the remote control station, the video signal flickers due to this interference.

- The latch that holds the WTC to the TTV or the WTC lid closed should be exchanged for a three position latch so that it can hold both the lid closed and the WTC on to the TTV at the same time.

- The TTV needs to have a remote starter relay to enable the vehicle to be started remotely.

- The TTV could be upgraded to a self-guided vehicle. This would reduce operating costs.

- An overview camera should be used to provide the TTV and REMEX operators with additional information for navigation and operations. The TTV cameras should be upgraded for a wider field of view and faster pan and tilt functions. A dual screen should be implemented on the TTV display so that the TTV and overview cameras could both be seen at the same time.

- The TTV and REMEX control systems should employ fine control (smaller overall movements) on all functions.

- When the TTV forward camera was switched on, the TTV joystick responded to up motion with a forward movement of the TTV. However, when the TTV reverse camera was switched on, the TTV responded to an upward motion of the joystick by traveling backward. The software should be corrected so that the TTV responds in the same direction from the operator's perspective whichever camera is activated.

- Dark lines were added to the WTC to aid in ITM placement just before the FY-94 demonstration. It would be helpful to add improved dark lines/targets to aid in ITM placement.

- The simulated roller table on the TTV should be modified to a functional roller table for an actual retrieval. 
- If the site specific application allows, a paved floor for the TTV should be used to control dust generation and potential contamination spread.

\subsubsection{REMEX}

- The REMEX successfully integrated with the IEE to successfully retrieve and transfer the simulated waste. However, the REMEX capacity was marginal. For example, while transferring a full ITM at full boom and stick extension, the back half of the REMEX tracks would lift off of the ground. Using a larger capacity excavator would improve the range and ability to handle waste forms at full extension.

- The excavator should be repaired so that the tracks provide equal power.

- The excavator cameras were not sealed for weather resistance. These cameras should be able to resist dust and inclement weather.

- The existing coordinated control of the REMEX arm should be tuned to handle the IEE and implemented.

- Coordinated control for the thumb should be developed and implemented.

- The repeated fuse failures on the REMEX during the FY-94 demonstration suggest that the signal distribution should be checked to ensure that the circuitry is adequate for the signal level. The short found in the ITM attach sensor could have been the cause of the repeated failures, so this may prove to be only a double check.

- Moving the ITM latch/lock and thumb control onto the joystick control arm would be helpful. A thumb operation with a rocker switch trigger and an ITM latch switch on top of the grip could be implemented. In addition, the REMEX remote control station hand grip should be replaced with a smaller, more comfortable unit such as the one in the REMEX cab.

- The REMEX cameras need pan and tilt capability to allow operators to view what they need to at all times. The cameras should also be upgraded for higher resolution and color views.

- For REMEX operations from the REMEX cab, a camera should be added to show the operator a view of the digface. (The digface view is obstructed by the IEE.)

\subsubsection{Overall Test Layout and Remote Control Station}

- Forklifts were used to remove the ITMs from the TTV and dump them during the FY-94 demonstration. An improved method for removing and processing the ITMs should be developed for future uses of this system.

- The communications radios should include a supervisor's override channel. With this feature, in the event of an emergency, the supervisor would not have to wait for other conversations to cease before providing direction and notification of the emergency. 
- A human factors designed remote control station should be designed and implemented for actual retrieval scenarios.

- Future trailer designs should take traffic patterns into account in the placement of the heating, ventilating, and air conditioning systems.

- Additional ITMs and WTCs should be placed near the excavator to increase excavation flexibility and efficiency. The spare ITMs could be used to load individual objects such as drums and cable, while the WTCs could be used to load large objects such as $4 \times 4 \times 8$-ft boxes and pick-up truck beds.

- For an actual retrieval scenario, it would be useful to have an additional robotic arm that could assist with manipulating items such as loose wire.

\subsubsection{Theoretical/Overall}

- As changes and modifications are made to the system, care should be taken to ensure that the system strengths, in particular the system integration and overall solution approach are not lost.

- The possibility of converting to a backhoe configuration rather than the front shovel configuration used in FY-94 should be considered. The front shovel configuration provides the advantages of less dust generation because the dirt tends to fall into the bucket, and increased safety because the excavator is located belowgrade and does not have the risk of falling into the waste pit or into air pockets in the waste pit. The backhoe configuration provides advantages in contamination control because the operations can take place from an abovegrade location, limiting the vehicle traffic on the contaminated surfaces.

- Some thought should be given by the BWID and Environmental Restoration Programs to further develop these systems into self-guided vehicles versus developing a bridge crane or using railroad tracks. These other methods may further limit the spread of contamination. The current designs for the ITM/WTC would be directly applicable to modifications for either a crane or railed system.

- Multiple TTVs could be used to reduce the excavator wait time and further increase throughput in an actual retrieval scenario.

\subsubsection{Design Modifications for a TRU Environment}

The following information is based on observational input; a more detailed evaluation should be made by a qualified person trained in the TRU environmental field before modifying or deploying this equipment in a TRU environment. There is insufficient data regarding operating the equipment in an TRU environment to give a cost estimate at this time.

- All of the equipment should be rugged enough to handle all condition encountered in the TRU retrieval environment, as well as, conditions encountered during decontamination of the equipment. The decontamination process may consist of power washing or spraying with $\mathrm{CO}_{2}$. Therefore, all electrical and hydraulic connections need to be water proofed and rugged enough 
to handle high pressure water. Hydraulic tanks, hydraulic cylinders, and fuel tanks should also be water tight. In addition, the entire design should include smooth surfaces and eliminate nooks and crannies so that contamination is easy to remove.

- The reliability of the equipment is highly important. The equipment needs to operate continuously with minimal unscheduled maintenance delays. This reliability can be designed into the equipment. Examples of these designs are proper shielding for electronics and video systems, TRU resistant hydraulic seals and glands, replace hydraulic motors with electric motors, and replace diesel engine with battery operated electric power plant.

- With equipment designed for TRU environment, the maintenance duration and frequency should be minimized. Modifications would need to be made to allow for maintenance and repair by personnel wearing bubble suits. The maintenance schedule for the equipment during the Phase II demonstration was as follows: $q / w$ hour to grease the IEE pin joints and daily fuel filter changes for the TTV diesel engine ( $1 / 2$ hour). A dirty fuel tank was the cause of the daily fuel filter changes. With a new fuel tank, the daily fuel filter changes could be eliminated.

- The existing system has a back-up fail safe system. This system consist of a pendant to manually operate the TTV in case of a remote control mode failure. Additional backup systems that would allow the TTV to be removed from the retrieval area could be considered. System safety could be further increased by using electric motors as opposed to hydraulics because the hydraulic fluid could become contaminated by TRU waste.

- System health monitoring would be useful in a TRU environment. The diagnostics used for system health monitoring would inform the operators of the real-time operating conditions of the equipment. Examples of the type of diagnostics needed are engine revolutions per minute, engine oil pressure, engine coolant temperature, electronics temperature, vehicle speed, and position information. 


\section{REFERENCES}

1. D. A. Arrenholz, and J. L. Knight, A Brief Analysis and Description of Transuranic Wastes in the Subsurface Disposal Area of the Radioactive Management Complex at INEL, EGG-WTD-9438, EG\&G Idaho, Inc., February 1991.

2. L. C. Meyer, Transuranic Contamination Control Using Electrostatic Curtains (Proof-of-Principle Experiments), EGG-WTD-9336, EG\&G Idaho, Inc., November 1990.

3. D. J. Valentich, Full Scale Retrieval of Simulated Buried Transuranic Waste, EGG-WTD-10895, EG\&G Idaho, Inc., September 1993.

4. P. M. Rice, A. M. Smith, and R. Peterson, Test Plan for the Remote Conveyance and IEE Demonstration, EGG-WTD-11185, EG\&G Idaho, Inc., August 1994.

5. L. C. Meyer, et al., La-oxides as Tracers for $\mathrm{PuO}_{2}$ to Simulate Contaminated Aerosol Behavior, EGG-WTD-11161, EG\&G Idaho, Inc., April 1994.

6. EG\&G Idaho, Inc., Quality Manual.

7. EG\&G Idaho, Inc., Safety Manual. 


\section{Appendix A}

\section{Telerobotic Transport Vehicle Operator's Manual}




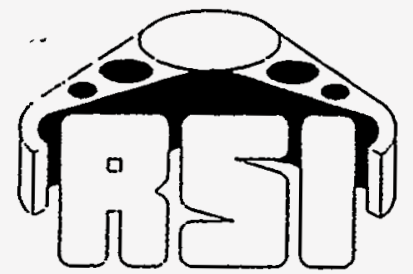

RSI Research Ltd

Project:

Document \#:

B3050 - Telerobotic Transport Vehicle

Title: 3050-000-MNL02

Author: Operator's Manual D. Lokhorst, B. Roy, W. Chin

\begin{tabular}{|c|c|c|c|c|l|}
\hline Rev. & Date & By & Chk. & Apr. & \\
\hline 0 & $6 / 2 / 94$ & DL & & & Release \\
\hline 1 & $12 / 1 / 94$ & BR & DoC- & AC & Issued for INEL demonstration report \\
\hline & & & & & \\
\hline & & & & & \\
\hline
\end{tabular}

1.0 Introduction 2

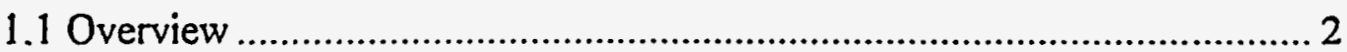

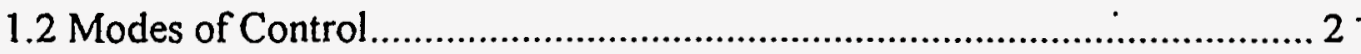

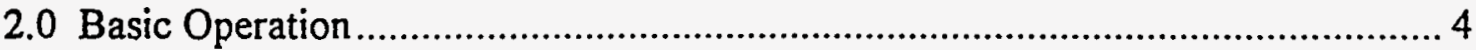

2.1 Capabilities of the Belly-pack ................................................................... 4

2.2 Connecting and Disconnecting the Belly-pack ............................................ 4

2.3 Step-by-step Startup Procedure............................................................... 4

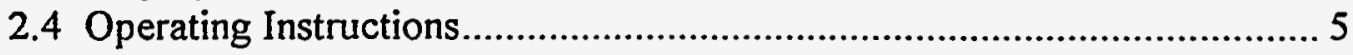

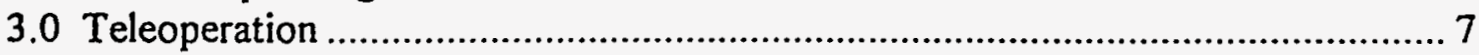

3.1 Teleoperator's Station Control Panel ....................................................... 7

3.2 Step-by-step Remote Operating Procedure................................................ 9

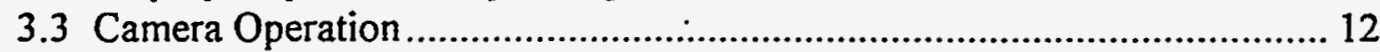

3.4 Special Features .................................................................................... 12 


\subsection{Introduction}

\subsection{Overview}

The Telerobotic Transport Vehicle (TTV) is provided with a computer control system. The vehicle can be operated from a "belly-pack" connected by cable to the vehicle, or from a remotely located control panel called the Teleoperator's Station.

This manual describes how to operate the TTV from both the belly-pack and from the teleoperator's station.

\subsection{Modes of Control}

The vehicle has three modes of control. It can be switched between these modes of control from the teleoperator's station. Descriptions of each mode are given below.

\section{Mode 1: Direct (Open Loop) Track Control}

This mode is the only mode available for use with the belly-pack. It is also available for remote controlled operation if Mode 2 control (described below) is not desired.

In this mode, sensory feedback is ignored by the automatic control system. The tracks are controlled in an open loop manner from the joystick controls (belly-pack or teleoperated via telemetry).

\section{Mode 2: Telerobotic (Closed Loop) Track Control}

This mode of operation is intended for use during normal remote controlled driving. Closed loop control of the tracks is provided. The vehicle responds to continuously updated desired linear and angular velocity commands received via telemetry. The on-board track speed sensors and the rate gyroscope are used for feedback to the servo control system.

In this mode, the ultrasonic range sensor array is monitored continuously. If the sensors indicate that objects are within preset ranges, a warning indicator will illuminate on the operator's console. These warning ranges are entered by keypad at the Teleoperator's Station. 


\section{Mode 3: Lid/Latch Control}

This mode of control is intended for use during loading and unloading, while the vehicle is stationary. Commands from the operator (via telemetry) will initiate the individual functions to be operated (e.g. lid open). The on-board control system will monitor the available limit switches for safety and to indicate completion of the task. 


\subsection{Basic Operation}

\subsection{Capabilities of the Belly-pack}

The TTV control system is provided with a belly-pack for controlling the tracks, lid, and latch. The belly-pack is intended to be used when it is not desirable to operate under remote control (when driving the TTV on or off a truck, for example).

The belly-pack is equipped with the following controls:

(1) a single 2-axis joystick for controlling the tracks

(1) a three position switch for controlling the latch

(1) a three position switch for controlling the lid

(1) an emergency stop switch

\subsection{Connecting and Disconnecting the Belly-pack}

WARNING! Turn the power OFF before connecting or disconnecting the belly-pack from the control box.

\section{Connecting the Belly-Pack}

1) Ensure that the key-switch is in the OFF position, i.e. ensure that power to the control system is disabled.

2) Connect the cable from the belly-pack to the connector on the TT.V electronics enclosure.

\section{Disconnecting the Belly-Pack}

1) Ensure that the key-switch is in the OFF position, i.e. ensure that power to the control system is disabled.

2) Disconnect the belly-pack connector from the control electronics enclosure on the TTV.

\subsection{Step-by-step Startup Procedure}

1) Connect the belly-pack as described above. 
2) Move the Mode Select switch (located on the TTV on-board electronics enclosure) to the OFF position.

3) Ensure that the Emergency Stop switch on the TTV is not depressed.

4) Ensure that the Emergency Stop switch on the belly-pack is not depressed.

5) Allow the track control lever to return to center.

6) Turn on the diesel engine if it is not already on.

7) Switch the key to the on position:

6) Move the Mode Select switch to the LOCAL position. The warning siren will activate as soon as the switch is placed in LOCAL. This indicates that the tracks are now active.

WARNING! All personnel, including the operator, should remain well clear of the TTV whenever the siren is activated.

7) The TTV is now under control of the belly-pack. Follow the instructions in the following section.

8) When the operation is complete, press the STOP button on the belly-pack to kill the engine. It is now safe to approach the machine.

\subsection{Operating Instructions}

\section{Stopping the Vehicle in an Emergency}

Whenever the belly-pack is connected, the red STOP switch is functional. Pressing the STOP switch will disable the hydraulic system by interrupting electrical power to the electrohydraulic control valves. Pressing the STOP switch will also kill the engine by activating a solenoid which will cut diesel fuel.

\section{Driving the Vehicle}

Use the joystick to drive the vehicle forward, reverse, and to turn left or right.

Forward travel corresponds to deflection of the joystick away from the operator. Reverse travel corresponds to deflection of the joystick toward the operator. The rate of turn of the vehicle corresponds to the deflection of the joystick to the left or right.

If at any time the TTV is out of control, releasing the joystick will allow it to automatically return to its neutral position stopping motion of the tracks. If a 
serious malfunction occurs immediately press the Hydraulics Off or Stop switch to abruptly disable TTV hydraulics and stop the engine.

\section{Opening and Closing the Lid and Latch}

Two switches are provided on the belly-pack for activating the lid and latch. The lid/latch switches should only be operated when the TTV is stopped.

WARNING! The lid and latch switches are active whenever the Control Mode Selector is in the LOCAL position. It is up to the operator to operate the lid controls in a safe manner. 


\subsection{Teleoperation}

\subsection{Teleoperator's Station Control Panel}

The Teleoperator's Station provides the operator with complete control of the TTV. The Teleoperator's Station is illustrated in RSI Research drawing 3050-400-001C. As shown in the drawing, there are controls for driving the vehicle, for moving the cameras, and for opening and closing the lid and latch. The following paragraphs give a brief overview of the various features of the control panel.

\section{Hydraulics Off Button}

The Hydraulics Off switch is intended to immediately put the vehicle into a safe state. When this switch is depressed, the hydraulic pump pressure is unloaded (i.e. sent to tank), and the power to the electrohydraulic valves is interrupted. This will instantly stop the vehicle. Unlike the on-board and belly-pack mounted Emergency Stop switches, the panel mounted Hydraulics Off button does not cut fuel to the diesel engine.

NOTE: In the event of a telemetry failure, the Hydraulics Off switch may not function correctly. Fail safety systems on the TTV itself will slow and stop the vehicle in the event of a telemetry failure.

\section{Control Mode Switch}

The type of control exercised by the operator from the teleoperator's station is selected by the Control Mode switch at the upper left of that console. For travel, the operator selects either direct track control or robotic track control. With the TTV stopped the operator selects lid/latch control to open and close the container and facilitate loading and unloading of the ITM.

\section{Track Control Joystick}

The motion of the TTV tracks is controlled using the large joystick at lower right on the Remote Console. TTV forward and reverse motion is caused by forward and backward motion of the stick while angular motion is caused by left and right motion of the stick. The joystick is calibrated through the keypad by a technician. Joystick deadband should be edited if necessary prior to calibration. 


\section{The LCD Display}

The teleoperator's station is equipped with a 4 row by 40 column liquid crystal display (LCD). A typical display is shown below:

\begin{tabular}{|llllll|}
\hline Status: OK & \multicolumn{3}{c}{ front } & \multicolumn{2}{c}{ rear } \\
LTrack: -22 & Range: 20 & 12 & 347 & 347 \\
RTrack: +136 & Alarm: 120 & 120 & 200 & 200 \\
Vehicle in telerobotic control & & \\
\hline
\end{tabular}

There are several types of data displayed. The field labeled "Status" displays the current operating status of the control system.

The values beside the "LTrack" and "RTrack" labels display the current speed of the left and right tracks, respectively, in $\mathrm{ft} / \mathrm{min}$.

The values beside "Range" display the current readings from the four ultrasonic sensors. The values beside "Alarm" indicate the current settings of ranges at which the collision avoidance system will be activated.

The bottom line of the display shows status information and operator messages.

\section{The Keypad}

The teleoperator's station is provided with a 16-button keypad. The keypad has the layout shown below:

$\begin{array}{llll}1 & 2 & 3 & \text { CLR } \\ 4 & 5 & 6 & <- \\ 7 & 8 & 9 & \rightarrow \\ * & 0 & \# & \text { ENTR }\end{array}$

The keypad is used to enter range set points for the acoustic sensor array. It is also uscd extensively in Technician's Mode, for diagnostics and calibration.

\section{Indicators and Warnings}

The control panel has several indicators and warnings, including: 
1) Lid (Open / Lid Closed indicators - indicates the state of the limit switches on the container lid.

2) Latch Open / Latch Closed indicators - indicate the state of the latch limit switches.

3) Alarm indicator - indicates the presence of objects to be avoided.

\section{Camera Controls}

The two TTV cameras are controlled using the cluster of controls on the lower left side of the Remote Console.

1) Select - Either the front or rear camera is selected using the select switch and subsequent pan, tilt zoom and focus changes will apply only to that camera.

2) Pan and Tilt - The pan and tilt joystick determines the horizontal and vertical angle while the zoom and focus change those functions on the selected camera.

3) Zoom - Shows the selected camera image at greater or lesser magnification.

4) Focus - Adjusts the selected camera image focus from far to near.

\subsection{Step-by-step Remote Operating Procedure}

\section{Preparation of the Remote Station}

1) Move the Control Mode select switch to the OFF position.

2) Ensure that the Hydraulics Off switch is not depressed.

3) Make sure that the joystick is allowed to return to center.

4) Turn the power switch on. Verify that the POWER indicator light is on.

5) Enter the 4-digit operator password at the LCD prompt, and press ENTR on the keypad. If this is successful, the display will be similar to the following:

\begin{tabular}{|lrlrrrr|}
\hline Status: & OK & & front & \multicolumn{2}{c|}{ rear } \\
LTrack: & 0 & Range: & 20 & 12 & 350 & 350 \\
RIrack: & 0 & Alarm: & 120 & 120 & 200 & 200 \\
Remote & Control & OFF & & & & \\
\hline
\end{tabular}

\section{Preparation of the Vehicle}

1) Move the Mode Select Switch (located on the TTV on-board electronics enclosure) to the OFF position. 
2) If attached, discomnect the belly-pack from the TTV, following the instruction in Section 2.2.

3) Ensure that the Emergency Stop switch on the TTV is not depressed.

4) Perform a mechanical inspection of the vehicle. Start the diesel engine, and wait the appropriate warm-up period.

5) To enable the remote control system, move the Mode Select Switch to the REMOTE position. At this time, the amber warning light should begin to flash.

WARNING! When the amber light is flashing, the remote station has been enabled. When the warning siren is heard, it indicates that the TTV is under active control from the remote station. Immediately move away from the vehicle.

\section{Driving the Vehicle: Mode 1 - Direct Track Control}

1) Verify that the REMOTE ENABLED indicator is lit. This will be on as soon as telemetry to the teleoperator's station verifies that the Mode Select switch on the TTV is in the REMOTE position.

2) Move the Control Mode select switch to the Direct Track position. The TTV is now under remote direct track operation.

3) Forward travel corresponds to deflection of the joystick away from the operator. Reverse travel corresponds to deflection of the joystick toward the operator. The direction of travel of the vehicle corresponds to the deflection of the joystick to the left or right.

Because direct track control has no feedback component the amount of deviation of the joystick is directly related to the amount of signal to the servo valves controlling the track motors. Typically, the tracks do not operate identically. To accommodate the lead or lag of one track over the other, the operator must use some sideways deflection to achieve a straight line path. Engine overload is also largely left up to the operator in this mode.

At any time, releasing the joystick will allow it to automatically return to its neutral position stopping motion of the tracks. If a serious malfunction occurs immediately press the Hydraulics Off switch to abruptly disable TTV hydraulics.

During operation monitor the alarm light and the LCD display for the presence of objects, abnormal telemetry conditions or fault status of the container's lid and latch. 


\section{Driving the Vehicle: Mode 2 - Telerobotic Track Control}

Driving the TTV in Telerobotic mode is in most ways similar to driving it in Direct Track mode.

1) Once the REMOTE ENABLED indicator is lit and telemetry is active, move the Control Mode switch to the Robotic Track position.

2) Joystick operation is similar to Direct Track control.

In Robotic Track control the deflection of the stick indicates a desired velocity, angular and linear. The on-board electronic system implements a control loop which achieves this velocity by applying the amount of output signal to the track valves and monitoring the resultant track velocity reported by the encoders on each track until the desired matches the actual velocity. This makes straight line travel and controlled turning much easier and direct.

Confirmation of the operation of the vehicle occurs primarily through use of the cameras. Typically the cameras are set in the most suitable position for straight line travel using the pan and tilt controls.

The operator must regularly monitor the warning alarm light and the LCD display on the Remote Console for indications of system faults and obstacle reports from the sonic sensors on the TTV.

\section{Handling the Container: Mode 3 - Lid/Latch Control}

1) Verify that the REMOTE ENABLED indicator is lit. This will be on as soon as telemetry to the teleoperator's station verifies that the Mode Select switch on the TTV is in the REMOTE position.

2) Move the Control Mode select switch to the Lid/Latch position. The lid and latch of the TTV are now under remote control.

3) The lid and latch can be controlled by the switches located in the TTV Control group on the teleoperator's control panel.

Above and below each switch are lights indicating the current state of the device controlled by each switch. No interlock logic prevents moving the lid when the latch is locked or locking the latch with the lid open and then closing the lid... safe and consistent handling is up to the operator.

When shutting the lid if the closed light does not come on, the container lid is not considered secure and the latch may not lock. Similarly if the locked light does not come on after switching the latch to locked the operator should assume that the locking mechanism is not fully engaged and operate accordingly. 


\subsection{Camera Operation}

Note: The camera pan \& tilt and zoom/focus functions will operate only if the REMOTE ENABLED indicator is on.

Select either the front or rear camera using the select switch of the camera controls at lower left on the Remote Console. The controls will activate only the camera currently selected.

Move the camera horizontally by moving the Pan \& Tilt control left or right.

Move the camera vertically by moving the Pan \& Tilt forward and back.

Zoom in or out by holding the momentary contact zoom switch in the in or out position until the desired video image is centered.

Adjust the camera focus by holding the focus switch in its far or near position until the video image is clear.

\subsection{Speciai Features}

\section{Acoustic Range Sensors for Collision Avoidance}

The .TTV is equipped with four ultrasonic sensors. Two are located on the front of the vehicle pointing forward, and two are located on the rear of the vehicle pointing rearward. These sensors measure the distance to any object in their direct path. The distances measured (in feet) are shown on the LCD display, beside the label "Range:".

The LC.D displays a second set of numbers, labeled "Alarm:". While operating in Mode 2 - Teleorobotic Control, the vehicle will stop if objects are detected within these limits. These values can be changed at any time from the control panel.

The procedure for changing the alarm range is as follows:

1) Use the increment $(+)$ or decrement $(-)$ keys to move between the fields in the alarm row. When a displayed number blinks, it can be edited.

2) Key in the desired range, in feet. Fractions are not accepted.

3) Press the ENTR key to enter the value that has been keyed in. Or press CLR to erase the new value and restore the old. 

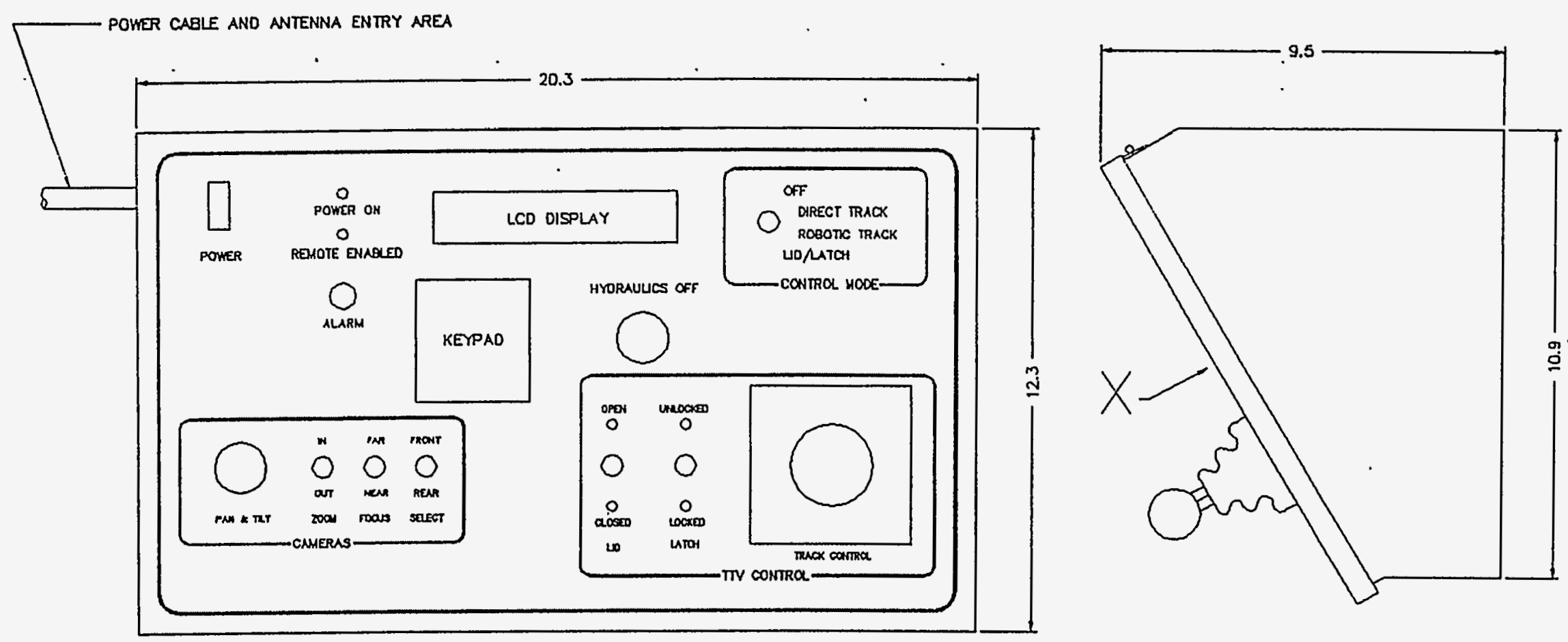

VIEW ON $X$

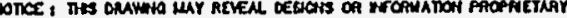

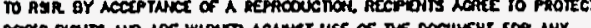

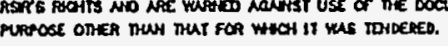

\begin{tabular}{|c|c|c|c|c|}
\hline \multirow{2}{*}{\multicolumn{2}{|c|}{ QVensors ARE $\mathrm{H}$ IH. }} & & & \multirow[b]{2}{*}{ CHWCE } \\
\hline & & & YN/D & \\
\hline \multirow{3}{*}{ 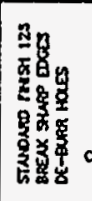 } & \multirow{3}{*}{ 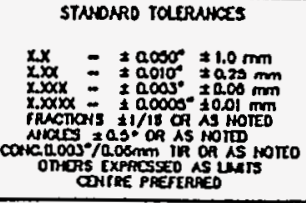 } & 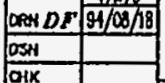 & & RSI RESEARCH LTD \\
\hline & & $\begin{array}{r}\text { Prover } \\
3050 \\
\end{array}$ & REMC & CONSOLE \\
\hline & & 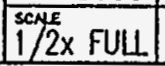 & 1 OF 1 & $3050-400-001 \mathrm{C}$ \\
\hline
\end{tabular}




\section{Appendix B}

Final Acceptance Test Records 



\begin{tabular}{|c|c|c|c|c|c|c|c|}
\hline \multicolumn{7}{|c|}{ Final Acceptance Test of TTV Control and Vision Systems } & \multirow{3}{*}{ 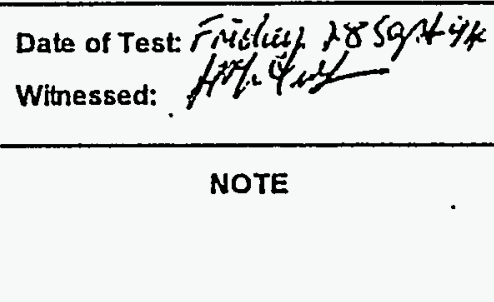 } \\
\hline \multirow[t]{2}{*}{ ITEM } & \multirow{2}{*}{$\begin{array}{l}\text { COMPONENT } \\
\text { OR SYSTEM }\end{array}$} & \multirow[t]{2}{*}{ TEST DESCRIPTION } & \multicolumn{2}{|c|}{ ACCEPT } & \multirow[t]{2}{*}{ ACTION } & \multirow[t]{2}{*}{ INIT } & \\
\hline & & & YES & No & & & \\
\hline \multirow[t]{14}{*}{ 4.3.1 } & $\begin{array}{l}\text { TTV Hydraulic } \\
\text { System }\end{array}$ & Quality of Parts (Documentation) & & & & & $\begin{array}{l}\text { Parts which have not previously } \\
\text { been covered should have } Q A \\
\text { papers }\end{array}$ \\
\hline & & Visual Quallty of Workmanship & & & & & Demonstrate using pendant controls \\
\hline & & - Hydraulle hoses, couplings \& supports & $\checkmark$ & & & & \multirow{5}{*}{$\begin{array}{l}\text { Prototypical plumbling \& system } \\
\text { configuration is noted }\end{array}$} \\
\hline & & - Valves, Manifolds \& Mounting blocks & $\checkmark$ & & & & \\
\hline & & - Slgnal and control wirlng & $\checkmark$ & & & & \\
\hline & & - Lid cylinders & $\checkmark$ & & & ' & \\
\hline & & - Lalch cyllinders & $\checkmark$ & & & & \\
\hline & & \multicolumn{2}{|l|}{ Function Test of Hydraulics } & & & & \\
\hline & & - TTV Forward & $\checkmark$ & & & & \\
\hline & & - TTV Reverse & $\checkmark$ & & & & \\
\hline & & - RH Track Forward & $\checkmark$ & & & & Could not control each track \\
\hline & & - RH Track Reverse & $\checkmark$ & & & & $\begin{array}{l}\text { separately since the control system } \\
\text { is issuing the command. }\end{array}$ \\
\hline & & - LH Track Fonward & $\checkmark$ & & & & Proportional control due to single \\
\hline & & - LH Track Reverso & $\checkmark$ & & & & controls). \\
\hline
\end{tabular}




\begin{tabular}{|c|c|c|c|c|c|c|c|}
\hline \multicolumn{7}{|c|}{ Final Acceptance Test of TTV Control and Vision Systems } & \multirow{3}{*}{ 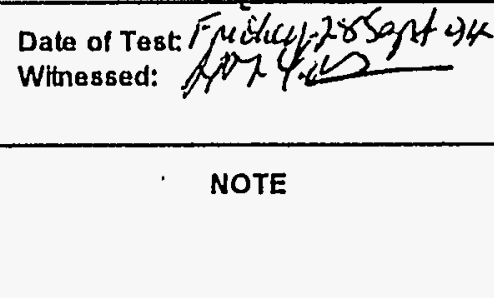 } \\
\hline \multirow[t]{2}{*}{ ITEM } & \multirow{2}{*}{$\begin{array}{l}\text { COMPONENT } \\
\text { OR SYSTEM }\end{array}$} & \multirow[t]{2}{*}{ TEST DESCRIPTION } & \multicolumn{2}{|c|}{ ACCEPT } & \multirow[t]{2}{*}{ ACTION } & \multirow[t]{2}{*}{ INIT } & \\
\hline & & & YES & No & & & \\
\hline 4.3.2 & $\begin{array}{l}\text { TTV Sensor } \\
\text { Systems }\end{array}$ & & & & & & \\
\hline \multirow[t]{8}{*}{ 4.3.2.1 } & - Velocity Sensors & Quality of components (Documentation) & & , & & & $\begin{array}{l}\text { Parts which have not previously } \\
\text { been covered should have all QA } \\
\text { papers provided }\end{array}$ \\
\hline & & Visual Quality of Workmanshlp & & & & & \\
\hline & & LEFT HAND & & & & & \\
\hline & & - Encoders and Encoder mounts & $\checkmark$ & & & & \\
\hline & & - Slgnal wire runs and fixings $\mathrm{LH}$ side & $\checkmark$ & & & & \\
\hline & & RIGHT HAND & & & & & \\
\hline & & - Encoder and Encoder mounts & $\checkmark$ & & & & \\
\hline & & - Signal wire runs and fixings RH side & $\checkmark$ & & & & \\
\hline
\end{tabular}




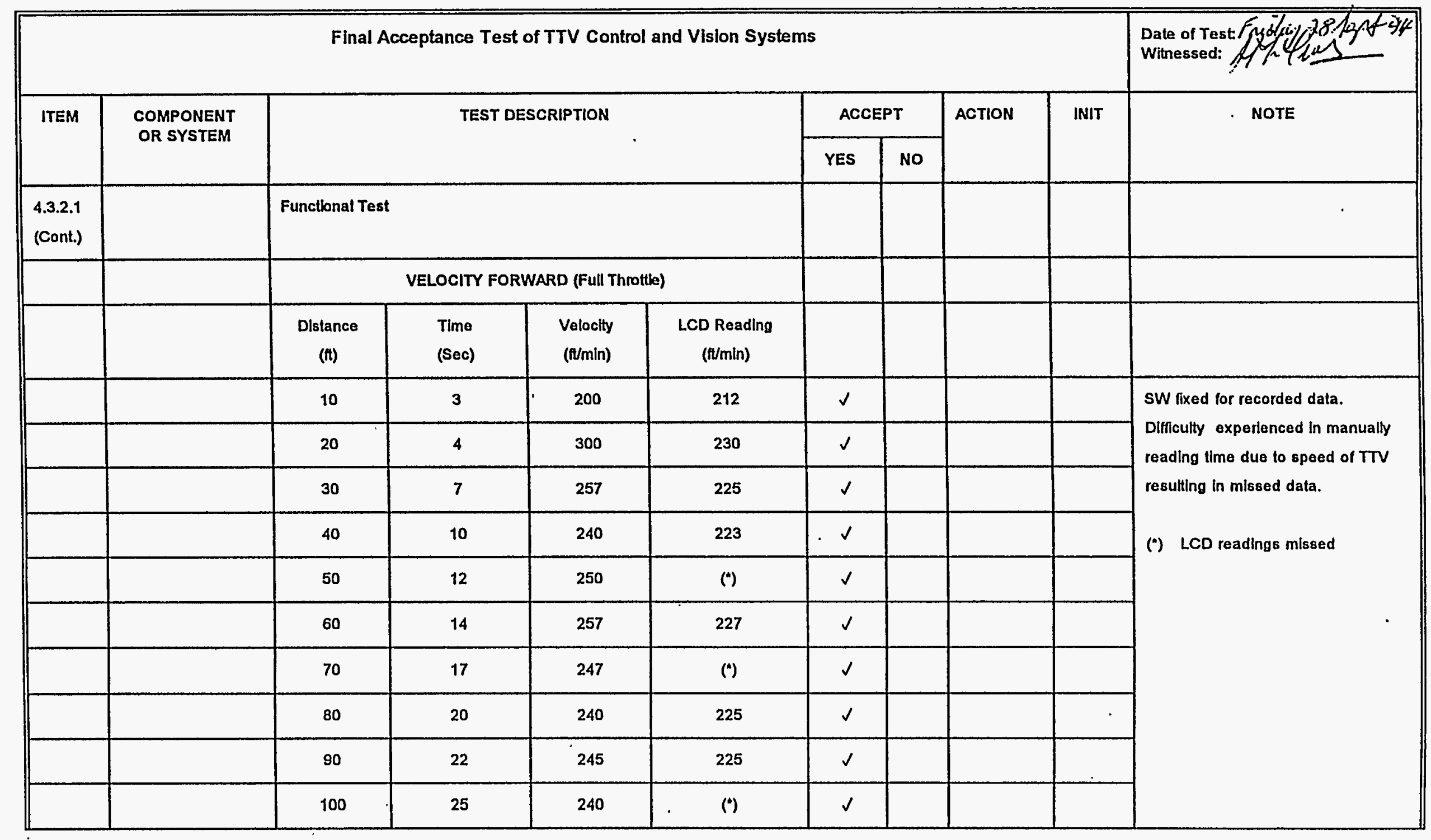




\begin{tabular}{|c|c|c|c|c|c|c|c|c|c|c|}
\hline \multicolumn{10}{|c|}{ Final Acceptance Test of TTV Control and Vision Systems } & \multirow{3}{*}{ 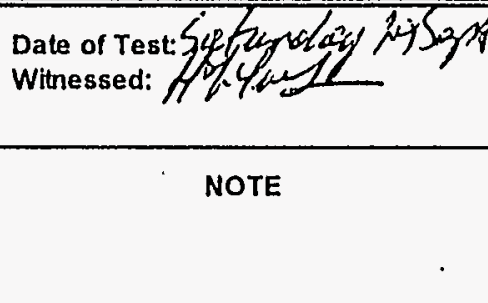 } \\
\hline \multirow[t]{2}{*}{ ITEM } & \multirow{2}{*}{$\begin{array}{l}\text { COMPONENT } \\
\text { OR SYSTEM }\end{array}$} & \multicolumn{4}{|c|}{ TEST DESCRIPTION } & \multicolumn{2}{|c|}{ ACCEPT } & \multirow[t]{2}{*}{ ACTION } & \multirow[t]{2}{*}{ INIT } & \\
\hline & & & & & & YES & No & & & \\
\hline \multirow{12}{*}{$\begin{array}{l}4.3 .2 .1 \\
\text { (Cont.) }\end{array}$} & & \multicolumn{4}{|c|}{ VELOCITY REVERSE (Part Throttle) } & & & & & \\
\hline & & $\begin{array}{c}\text { Distance } \\
\text { (it) }\end{array}$ & $\begin{array}{l}\text { TIme } \\
\text { (Sec) }\end{array}$ & $\begin{array}{l}\text { Velocity } \\
\text { (tt/min) }\end{array}$ & $\begin{array}{l}\text { LCD Reading } \\
\quad(\mathrm{f} / \mathrm{m} / \mathrm{n})\end{array}$ & & & & & \\
\hline & & 10 & 4 & 150 & 164 & $\checkmark$ & & & & \\
\hline & & 20 & 7 & 171 & 161 & $\checkmark$ & & & & \\
\hline & & 30 & 10 & 180 & 167 & $\checkmark$ & & & & \\
\hline & & 40 & 13 & 185 & 165 & $\checkmark$ & & & & \\
\hline & & 50 & 17 & 176 & 167 & $\checkmark$ & & & & \\
\hline & & 60 & 20 & 180 & 170 & $\checkmark$ & & & & \\
\hline & & 70 & 23 & 183 & 165 & $\checkmark$ & & & & \\
\hline & & 80 & 26 & 185 & 164 & $\checkmark$ & & & & \\
\hline & & 90 & 29 & 186 & 168 & $\checkmark$ & & & & \\
\hline & & 100 & 39 & 154 & 161 & $\checkmark$ & & & & \\
\hline
\end{tabular}




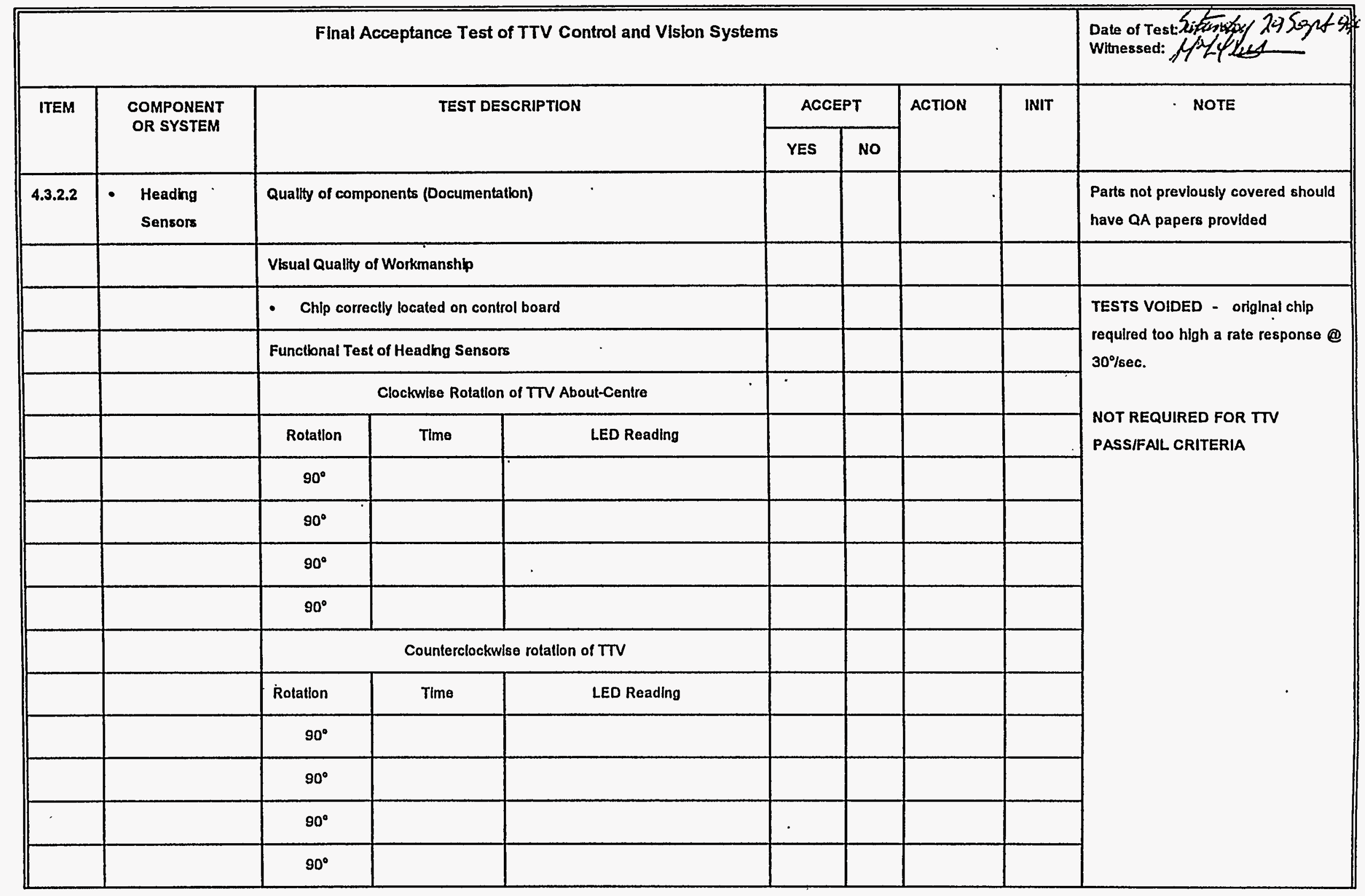

$$
\text { III - } 6
$$




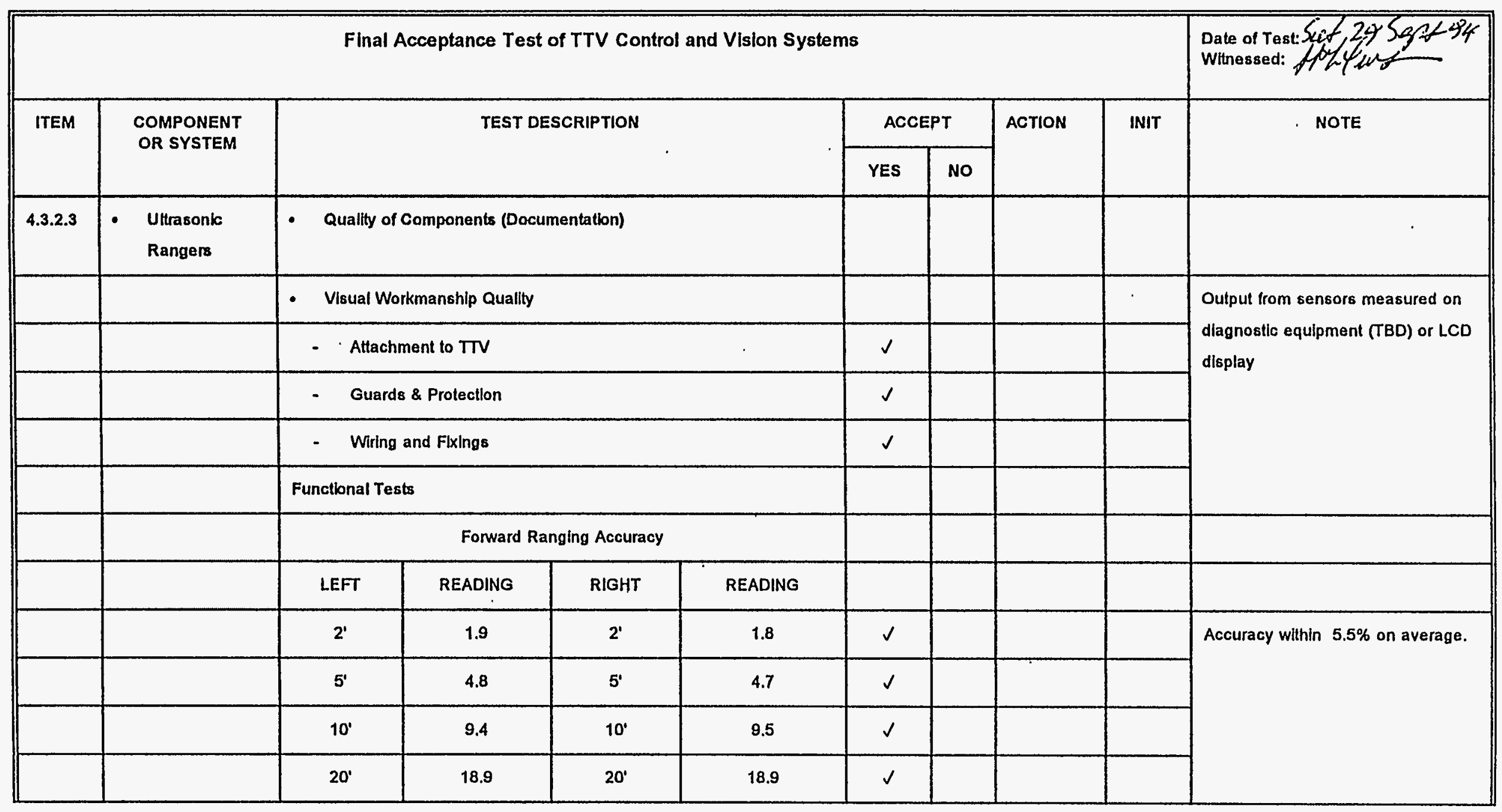




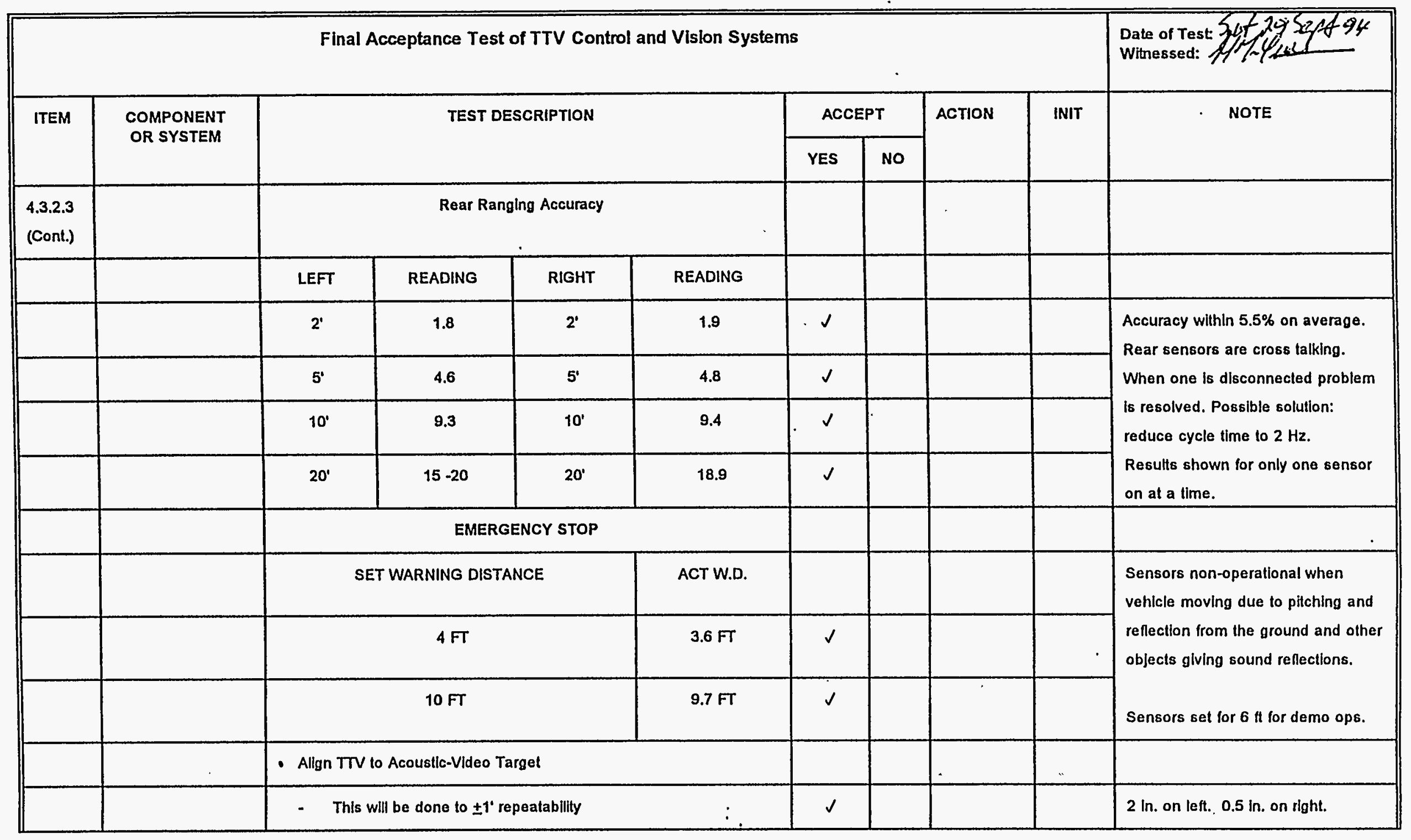




\begin{tabular}{|c|c|c|c|c|c|c|c|}
\hline \multicolumn{7}{|c|}{ Final Acceptance Test of TTV Control and Vision Systems } & \multirow{3}{*}{ 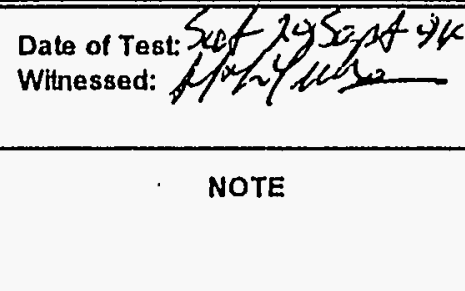 } \\
\hline \multirow[t]{2}{*}{ ITEM } & \multirow{2}{*}{$\begin{array}{l}\text { COMPONENT } \\
\text { OR SYSTEM }\end{array}$} & \multirow[t]{2}{*}{ TEST DESCRIPTION } & \multicolumn{2}{|c|}{ ACCEPT } & \multirow[t]{2}{*}{ ACTION } & \multirow[t]{2}{*}{ INIT } & \\
\hline & & & YES & No & & & \\
\hline \multirow[t]{12}{*}{ 4.3.2.4 } & - Limit Switches & Visual Quallty of Workmanship & & & & & \\
\hline & & - LID open/close & $\checkmark$ & & & & \\
\hline & & - Mounting and fixing of microswitch & $\checkmark$ & & & & \\
\hline & & - Wiring of microswitch and fasteners & $\checkmark$ & & & & \\
\hline & & - Latch open/close & $\checkmark$ & & & & \\
\hline & & - Mounting and fixlng of mircoswitch & $\checkmark$ & & & & \\
\hline & . & - Wiring of mircroswittch and fastening & $\checkmark$ & & & & \\
\hline & & Functional Test & & & & & - \\
\hline & & - Lid open/close & $\checkmark$ & & & & \\
\hline & & - Indicators displayed at panel & $\checkmark$ & & & & \\
\hline & & - Latch lock/unlock & $\checkmark$ & & & & \\
\hline & & - Indicators correctly display status at panel & $\checkmark$ & & & & \\
\hline
\end{tabular}




\begin{tabular}{|c|c|c|c|c|c|c|c|}
\hline \multicolumn{7}{|c|}{ Final Acceptance Test of TTV Control and Vision Systems } & \multirow{3}{*}{ 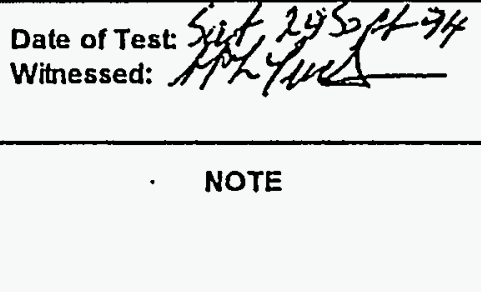 } \\
\hline \multirow[t]{2}{*}{ ITEM } & \multirow{2}{*}{$\begin{array}{l}\text { COMPONENT } \\
\text { OR SYSTEM }\end{array}$} & \multirow[t]{2}{*}{ TEST DESCRIPTION } & \multicolumn{2}{|c|}{ ACCEPT } & \multirow[t]{2}{*}{ ACTION } & \multirow[t]{2}{*}{ INIT } & \\
\hline & & & YES & No & & & \\
\hline \multirow[t]{15}{*}{ 4.3.2.5 } & $\begin{array}{l}\text { - Onboand control } \\
\text { HMW }\end{array}$ & Quality of components (Documentation) & & & & & $\begin{array}{l}\text { Documents: Those not already } \\
\text { covered will be provided }\end{array}$ \\
\hline & & Visual Qualty of workmanship & & & & & \\
\hline & . & - enclosure and attachment to TV & $\checkmark$ & & & & \\
\hline & & - closure devlce and lock & $\checkmark$ & & & & \\
\hline & & - control electronlc board \& attachments & $\checkmark$ & & & & Good quality components \\
\hline & & - cables/signal wires and fixtures & $\checkmark$ & & & & \\
\hline & & - Connectors & $\checkmark$ & & & & \\
\hline & & - Antenna and Protecllve Dome & $\checkmark$ & & & & \\
\hline & & Functional Trials & & & & & $\begin{array}{l}\text { Using pendant controls. Diferential } \\
\text { skid steering test falled due to track } \\
\text { locking on In turn. }\end{array}$ \\
\hline & & - TTV Forward & $\checkmark$ & & & & \\
\hline & & - TTV Reverse & $\checkmark$ & & & & \\
\hline & & - Rlght Track Forward & $\checkmark$ & & & & Joystick at $45^{\circ}$ \\
\hline & & - Rlght Track Reverse & $\checkmark$ & & & & \\
\hline & & - Left Track Forward & $\checkmark$ & & & & \\
\hline & & - Left Track Reverse & $\checkmark$ & & & & \\
\hline
\end{tabular}

III -10 


\begin{tabular}{|c|c|c|c|c|c|c|c|}
\hline \multicolumn{7}{|c|}{ Final Acceptance Test of TTV Control and Vision Systems } & \multirow{3}{*}{ 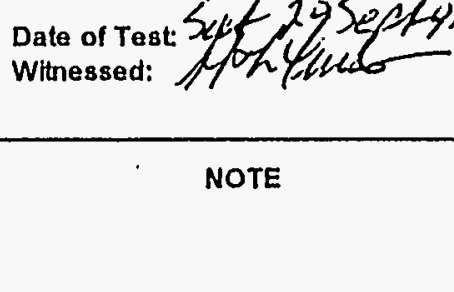 } \\
\hline \multirow[t]{2}{*}{ ITEM } & \multirow{2}{*}{$\begin{array}{l}\text { COMPONENT } \\
\text { OR SYSTEM }\end{array}$} & \multirow[t]{2}{*}{ TEST DESCRIPTION } & \multicolumn{2}{|c|}{ ACCEPT } & \multirow[t]{2}{*}{ ACTION } & \multirow[t]{2}{*}{ INIT } & \\
\hline & & & YES & NO & & & \\
\hline \multirow{5}{*}{$\begin{array}{l}4.3 .2 .5 \\
\text { (Cont.) }\end{array}$} & & - Emergency Stop & $\checkmark$ & & & & \\
\hline & & - Valve disable & $\checkmark$ & & & & \\
\hline & & - Fuel off & $\checkmark$ & & & & \\
\hline & & - Lid up/down & $\checkmark$ & & & & \\
\hline & & - Latch on/off & $\checkmark$ & & & & \\
\hline \multirow[t]{6}{*}{ 4.3.2.6 } & $\begin{array}{l}\text { TTV Vision System } \\
\text { (TV Remote } \\
\text { Transmission) }\end{array}$ & - Quality of Components & & & & & $\begin{array}{l}\text { If not covered by other tests, QA } \\
\text { papers will be provided. }\end{array}$ \\
\hline & & - Visual Inspection of Workmanship. & $\checkmark$ & & & & \\
\hline & & - Front camera & & & & & \\
\hline & & $\begin{array}{l}\text { Fastening of panfilt to TTV cables runs } \\
\text { and attachment }\end{array}$ & $\checkmark$ & & & & $\begin{array}{l}\text { Foam needed to put between } \\
\text { housing and camera to reduce } \\
\text { vibration }\end{array}$ \\
\hline & & - Rear camera & & & & & \\
\hline & & $\begin{array}{l}\text { Fastening of pan/till to TTV cables, runs } \\
\text { and attachment }\end{array}$ & $\checkmark$ & & & & \\
\hline
\end{tabular}




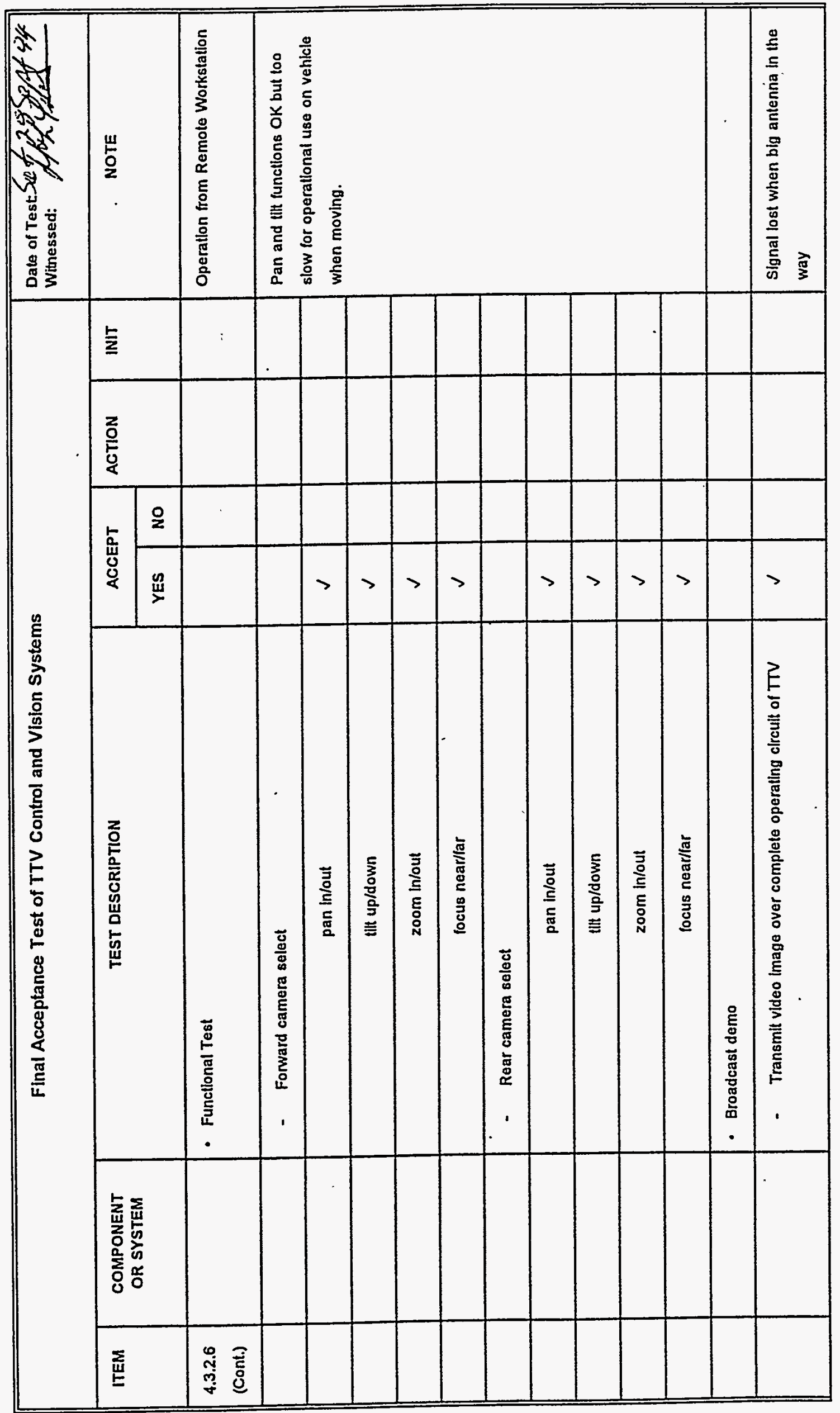




\begin{tabular}{|c|c|c|c|c|c|c|c|}
\hline \multicolumn{7}{|c|}{ Final Acceptance Test of TTV Control and VIsion Systems } & \multirow{3}{*}{ 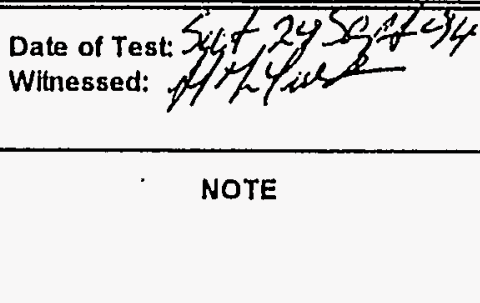 } \\
\hline \multirow[t]{2}{*}{ ITEM } & \multirow{2}{*}{$\begin{array}{l}\text { COMPONENT } \\
\text { OR SYSTEM }\end{array}$} & \multirow[t]{2}{*}{ TEST DESCRIPTION } & \multicolumn{2}{|c|}{ ACCEPT } & \multirow[t]{2}{*}{ ACTION } & \multirow[t]{2}{*}{ INIT } & \\
\hline & & & YES & No & & & \\
\hline \multirow[t]{12}{*}{ 4.3.2.7 } & $\begin{array}{l}\text { - Tele Operator } \\
\text { Station } \\
\text { (excluding } \\
\text { vislon) }\end{array}$ & - Quality of components & & & & & Document or parts to be provided \\
\hline & & - Visual Inspection of workmanshlp & & & & & Layout needs revlew. \\
\hline & & - enclosure and fàsteners & $\checkmark$ & & & & \\
\hline & & - power switch & $\checkmark$ & & & & \\
\hline & & - power on Indlcator & $\checkmark$ & & & & \\
\hline & & - remote enables & $\checkmark$ & & & & \\
\hline & & . emergency swilch & $\checkmark$ & & & & \\
\hline & & - control mode selector & $\checkmark$ & & & & \\
\hline & & - $\quad$ LCD displays & $\checkmark$ & & & & LCD colour/contrast too close \\
\hline & & - key pad with alpha-numerics & $\checkmark$ & & & & \\
\hline & & - TV motton hand controller & $\checkmark$ & & & & \\
\hline & & - Camera controller & $\checkmark$ & & & & \\
\hline
\end{tabular}




\begin{tabular}{|c|c|c|c|c|c|c|c|}
\hline \multicolumn{7}{|c|}{ Final Acceptance Test of TTV Control and Vision Systems } & \multirow{3}{*}{ 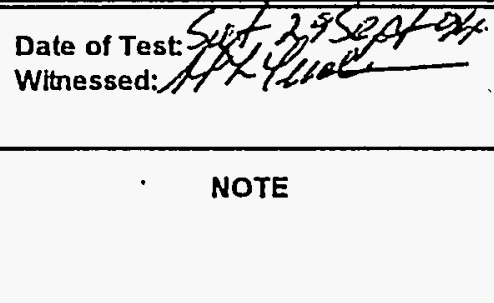 } \\
\hline \multirow[t]{2}{*}{ ITEM } & \multirow{2}{*}{$\begin{array}{l}\text { COMPONENT } \\
\text { OR SYSTEM }\end{array}$} & \multirow[t]{2}{*}{ TEST DESCRIPTION } & \multicolumn{2}{|c|}{ ACCEPT } & \multirow[t]{2}{*}{ ACTION } & \multirow[t]{2}{*}{ INIT } & \\
\hline & & & YES & No & & & \\
\hline \multirow{13}{*}{$\begin{array}{l}4.3 .2 .7 \\
\text { (Cont.) }\end{array}$} & . & - Internal: & & & & & \\
\hline & & cables & $\checkmark$ & & & & . \\
\hline & & electronlc boards & $\checkmark$ & & & & \\
\hline & & connectors \& fasteners & $\checkmark$ & & & & \\
\hline & & - Functlonal test & & & & & Using RF link from within trailer \\
\hline & & - Check keypad dlagnostlc operation & $\checkmark$ & & & & \\
\hline & & - Lld indicator up/down & $\checkmark$ & & & & \\
\hline & & - Latch indlcator on/off & $\checkmark$ & & & & \\
\hline & . & - TV fonward/reverse & $\checkmark$ & & & & \\
\hline & & - TTV turn leftright & $\checkmark$ & & & & \\
\hline & & - emergency stop & $\checkmark$ & & & & \\
\hline & & - lid up/down & $\checkmark$ & & & & \\
\hline & & - latch open/close & $\checkmark$ & & & & \\
\hline
\end{tabular}




\begin{tabular}{|c|c|c|c|c|c|c|c|}
\hline \multicolumn{7}{|c|}{ Final Acceptance Test of TTV Control and Vislon Systems } & \multirow{3}{*}{$\begin{array}{l}\text { Date of Test: } \\
\text { Witnessed: }\end{array}$} \\
\hline \multirow[t]{2}{*}{ ITEM } & \multirow{2}{*}{$\begin{array}{l}\text { COMPONENT } \\
\text { OR SYSTEM }\end{array}$} & \multirow[t]{2}{*}{ TEST DESCRIPTION } & \multicolumn{2}{|c|}{ ACCEPT } & \multirow[t]{2}{*}{ ACTION } & \multirow[t]{2}{*}{ INIT } & \\
\hline & & & YES & No & & & \\
\hline \multirow[t]{2}{*}{ 4.3.2.8 } & $\begin{array}{l}\text { TTV Visions } \\
\text { Systems } \\
\text { - Acoustle } \\
\text { Postlioning }\end{array}$ & - Repeatabillty demo & & & & & \\
\hline & & $\begin{array}{l}\text { - using a combination of video and acoustic feedback demonstrate } \\
\text { the the TTV can be posilloned with a repeatability of } \pm 1 \mathrm{ft} \text {. }\end{array}$ & $\checkmark$ & & & & $\begin{array}{l}\text { Accurate to } 2 \mathrm{in.} \text { on left and } 0.5 \mathrm{ln} \text {. } \\
\text { on right sensor }\end{array}$ \\
\hline 4.3.2.9 & Software & Software will be implictly tested by carrying out the foregoing lests & & & & & \\
\hline
\end{tabular}


Appendix C

\section{Derivation of the Dust Level}



- 


\section{Appendix C}

\section{Derivation of the Dust Level}

This is a description of the data and process used to develop the dust level requirement for the Innovative End Effector/Dust Free Dumping Demonstration.

Given the following average* contamination level expected for RWMC remediation activities:

$450 \mathrm{nCi} / \mathrm{g}: \quad$ Plutonium/Americium/mass soil. Based on calculations done by Guy Loomis that showed a level of $150 \mathrm{nCi} / \mathrm{g}$; this number was increased by a factor of 3 when Hazel O'Leary announced (1993) the factor of 3 increase in the amount of plutonium at the RWMC. Local hot spots may have higher contamination levels.

To allow for bubble suit entry, it is necessary to have an airborne source term of $<10 \mathrm{nCi} / \mathrm{g}$. [Based on Maximum Permissible Concentration (MPC) values and calculated by Burt Baldwin and Wayne Scofield.] Bubble suited entry into areas of higher airborne source term has been shown to lead to uptake during doffing of bubble suits.

Problem: Need to reduce the amount of aerosolized dust so that the airborne source term is $<10 \mathrm{nCi} / \mathrm{g}$.

If we completely aerosolized the dust, we would see airborne source term of $450 \mathrm{nCj} / \mathrm{g}$. (In actuality, this is not possible, because some particles are too heavy to become/remain airborne. To be conservative, we will use this worst case number.) To achieve an airborne source term of $<10 \mathrm{nCi} / \mathrm{g}$, we need to remove $98 \%$ of the dust $(10 \mathrm{nCi} / \mathrm{g} / 450 \mathrm{nCi} / \mathrm{g}=.02$, or $2 \%)$.

The next step is to identify a number (mass per unit volume, i.e., $g / L$ ) that represents a worst case (100\% aerosolized) dust measurement. A study done at the INEL (Menkhaus, 1991) regarding shower effectiveness included a test that aerosolized dust to simulate a worst case scenario. In the test, dust was aerosolized continuously and air particulate filters were used to measure the amount of airborne dust. The amount (mass) of airborne dust per unit volume of air seen was $5.5 \mathrm{E}-3 \mathrm{~g} / \mathrm{L}$, as shown below.

\begin{tabular}{|c|c|}
\hline Flowrate: & $6.85 \mathrm{~L} / \mathrm{min}$ \\
\hline Test time: & 3 minutes \\
\hline Volume of air: & $20.55 \mathrm{~L}(6.85 \mathrm{~L} / \mathrm{min} \times 3 \mathrm{~min}=20.55 \mathrm{~L})$ \\
\hline Mass: & $\begin{array}{l}0.113 \mathrm{~g} \text { (Value taken from average of filters at three heights; } 2,4 \text {, and } 6-\mathrm{ft} \\
\text { above ground.) }\end{array}$ \\
\hline Aerosolized dust: & $5.5 \mathrm{E}-3 \mathrm{~g} / \mathrm{L}(.113 \mathrm{~g} / 20.55 \mathrm{~L}=.0055 \mathrm{~g} / \mathrm{L})$ \\
\hline
\end{tabular}

This number, $5.5 \mathrm{E}-3 \mathrm{~g} / \mathrm{L}$, represents worst case aerosolized dust. To achieve the required airborne source term, we need to achieve a $98 \%$ improvement over this value. The maximum dust level allowed is therefore:

$1.1 \mathrm{E}-4 \quad[.0055 \mathrm{~g} / \mathrm{L} \times .02=.00011 \mathrm{~g} / \mathrm{L}]$ 
This number (1.1E-4 $\mathrm{g} / \mathrm{L}$ ) will be used as the goal for the dust level to be achieved by the Innovative End Effector/Dust Free Dumping Demonstration to be conducted in FY-94.

* Note: Much higher values could be encountered for regions containing waste containers with the maximum amount of allowable plutonium/americium, i.e., $200 \mathrm{~g} /$ container. These areas constitute local hot spots.

\section{REFERENCES}

Menkhaus, Daniel E., "Personnel Protective Equipment Total-Encapsulating Suit Decontamination Study Using Shower-Systems,” EGG-WTD-9475, EG\&G Idaho, Inc., January 1991. 


\section{Appendix D}

\section{Chemistry Laboratory Report, Dust and Rare Earth Trace Analysis}





\section{Determination of Praseodymium in Air Particulate Samples Collected on Glass Fiber Filters}

\section{INTRODUCTION}

This report details the analysis of the air particulate filters for praseodymium (Pr) submitted to the chemistry lab on October 3, 1994. The samples were analyzed by Inductively Coupled PlasmaMass Spectrometry (ICP-MS) after digestion/dissolution with nitric acid $\left(\mathrm{HNO}_{3}\right)$ and hydrogen peroxide $\left(\mathrm{H}_{2} \mathrm{O}_{2}\right)$. The $\operatorname{Pr}$ results from the analysis of the air particulate filters are shown in Table D-3. The Table D-3 results have been corrected for the mean Pr/filter result from the analysis of the "blank" filters. The "blank" filter results are presented in Table D-2 and a summary of the calibration checks and other QA/QC are shown in Table D-4. A copy of the spreadsheet detailing the ICP-MS run-log and the subsequent calculations is provided as Appendix 1. 


\section{SAMPLE PREPARATION AND ANALYSIS}

The filters were digested in $250 \mathrm{~mL}$ teflon beakers precleaned by heating with a $50 \% \mathrm{HNO}_{3}$ solution. Using PVC gloved hands the filters were removed from the petri dishes and transferred into the beakers. When folding the filter for placement in the beaker, the loaded side was kept inward to prevent any particulate from being lost. Prior to digestion, $0.5 \mathrm{~mL}$ of the surrogate spiking solution containing $25 \mu \mathrm{g} / \mathrm{mL}$ of holmium (Ho) and thulium ( $\mathrm{Tm}$ ) was spiked onto each filter and allowed to dry. A $50 \mathrm{~mL}$ aliquot of $50 \% \mathrm{HNO}_{3}$ was added to each beaker. The beakers were covered with a watch glass, placed on a hot plate and heated to about $95^{\circ} \mathrm{C}$ and allowed to reflux for 45 minutes. The samples were removed from the hot plate and allowed to cool. A $3 \mathrm{~mL}$ aliquot of $30 \% \mathrm{H}_{2} \mathrm{O}_{2}$ was added to each sample. When the effervescing subsided the samples were placed back on the hot plate and more hydrogen peroxide was added to each sample until the reaction was minimized. The solutions were refluxed again for one hour, removed from the hot plate and allowed to cool. The samples were then filtered using disposable filtering units with a $0.45 \mu \mathrm{m}$ filter, quantitatively transferred to a $250 \mathrm{~mL}$ volumetric flask and diluted to volume with deionized water (resistance $\geq 18 \mathrm{Mohm}$ ). A $9.75 \mathrm{~mL}$ sample aliquot was pipetted into a sample tube and a $250 \mu \mathrm{l}$ aliquot of the internal standard spiking solution $(5 \mu \mathrm{g} / \mathrm{mL}$ In as the IS and $5 \mu \mathrm{g} / \mathrm{mL} \mathrm{La}$ as a "precision" standard) was added to each sample before analysis by ICP-MS.

The ICP-MS was operated in the peak jump mode with three 30 second data acquisitions covering $\mathrm{m} / \mathrm{z} 115,129,141,165$, and 169 for $\mathrm{In}, \mathrm{La}, \mathrm{Pr}, \mathrm{Ho}$, and $\mathrm{Tm}$, respectively. The averaged, integrated counts per second (cps) for $\mathrm{La}, \mathrm{Pr}, \mathrm{Ho}$, and $\mathrm{Tm}$ were ratioed to the cps for $\mathrm{In}$. Calibration curves were constructed for each analyte from standards containing the five elements at the concentrations listed in Table D-1. Each standard contained $5 \% \mathrm{HNO}_{3}$ and the aliquots of each analyte added to a particular standard were weighed to assure consistency.

Table D-1. Concentrations of analytes used to calibrate the ICP-MS for $\operatorname{Pr}$ analysis.

\begin{tabular}{cccccc}
\hline & \multicolumn{5}{c}{ Concentration $(\mathrm{ng} / \mathrm{mL})$} \\
\cline { 2 - 6 } Standard & In & La & Pr & Ho & Tm \\
\hline 1 & 100 & 0 & 0 & 0 & 0 \\
2 & 100 & 104 & 79 & 49 & 152 \\
3 & 100 & 152 & 159 & 101 & 52 \\
4 & 100 & 54 & 230 & 151 & 101 \\
5 & 100 & 201 & 312 & 198 & 201 \\
\hline
\end{tabular}




\section{ANALYSIS RESULTS}

The analysis results for seven blank filters are shown in Table D-2. An eighth blank filter was submitted for analysis but was lost when the volumetric flask containing the final dilution was broken. The $\mathrm{Pr}$ results for the filters containing air particulates are presented in Table D-3. The results have been corrected for background $\mathrm{Pr}$ by subtracting the mean $\mu \mathrm{g} \mathrm{Pr} /$ filter determined from the 7 blank filters (Table D-2) from the $\mu \mathrm{g}$ of $\mathrm{Pr}$ found on every actual sample filter. The concentration in $\mu \mathrm{g} / \mathrm{g}$ of $\mathrm{Pr}$ in the collected particulate matter was then simply calculated by dividing by the particulate mass. The actual particulate masses had been determined prior to submission of the samples to the chemistry lab for analysis. The earth's crust is $\sim 5.5 \mu \mathrm{g} \mathrm{Pr} / \mathrm{g}$ so any levels of Pr greater than 5.5-6 $\mu \mathrm{g}$ $\mathrm{Pr} / \mathrm{g}$ would indicate that $\mathrm{Pr}$ spiked dirt/dust had been collected. Every sample appears to contain at least 10 times the $\mathrm{Pr}$ as would be expected naturally. In general, the recovery of the Ho and Tm surrogates was quite good indicating that the general sample preparation process was reproducible. Reagent blanks and reagent blank spikes were also prepared and taken through the whole sample preparation process. The reagent blank and spike results are given in Table D-4.

Table D-2. Pr contained in "blank" glass fiber filters.

\begin{tabular}{cl}
\hline Blank Filter 1 & $0.104 \mu \mathrm{g} \mathrm{Pr} /$ filter \\
Blank Filter 2 & $0.098 \mu \mathrm{g} \mathrm{Pr} /$ filter \\
Blank Filter 3 & $0.102 \mu \mathrm{g} \mathrm{Pr} /$ filter \\
Blank Filter 4 & $0.114 \mu \mathrm{g} \mathrm{Pr/filter}$ \\
Blank Filter 5 & $0.103 \mu \mathrm{g} \mathrm{Pr/filter}$ \\
Blank Filter 6 & $0.113 \mu \mathrm{g} \mathrm{Pr/filter}$ \\
Blank Filter 8 & $0.110 \mu \mathrm{g} \mathrm{Pr/filter}$ \\
\hline Mean & $0.106 \mu \mathrm{g} \mathrm{Pr/filter}$ \\
\hline Standard Deviation & $0.006 \mu \mathrm{g} \mathrm{Pr/Filter}$ \\
\hline
\end{tabular}


Table D-3. Results of $\operatorname{Pr}$ analysis of air particulate collected on filters.

\begin{tabular}{|c|c|c|c|c|c|}
\hline Sample & $\operatorname{Pr} \mu \mathrm{g} /$ filter & $\operatorname{Pr} \mu \mathrm{g} / \mathrm{g}$ & Sample & $\operatorname{Pr} \mu \mathrm{g} /$ filter & $\operatorname{Pr} \mu \mathrm{g} / \mathrm{g}$ \\
\hline P-001 & 0.65 & 67.0 & P-029 & 43.3 & 540 \\
\hline P-002 & 1.76 & 57.1 & P-030 & 25.3 & 468 \\
\hline P-003 & 0.46 & 46.0 & P-031 & 51.8 & 624 \\
\hline P-004 & 84.2 & 1460 & P-032 & 35.7 & 676 \\
\hline P-005 & 96.4 & 713 & P-033 & 46.3 & 921 \\
\hline P-006 & 90.1 & 741 & P-034 & 13.1 & 695 \\
\hline P-007 & 230 & 1160 & P-035 & 53.4 & 1070 \\
\hline P-008 & 6.13 & 356 & P-036 & 97.3 & 1030 \\
\hline P-009 & 9.14 & 405 & P-037 & 82.2 & 859 \\
\hline P-010 & 190 & 1070 & P-038 & 16.9 & 599 \\
\hline P-011 & 446 & 888 & P-039 & 62.7 & 922 \\
\hline P-012 & 4.55 & 350 & P-040 & 40.1 & 1090 \\
\hline P-013 & 0.62 & 63.5 & P-041 & 63.5 & 1260 \\
\hline P-014 & 0.31 & 53.5 & P-042 & 1.41 & 148 \\
\hline P-015 & 98.5 & 1670 & P-043 & 0.98 & 102 \\
\hline P-016 & 40.1 & 1030 & P-044 & 1.09 & 118 \\
\hline P-017 & 103 & 1100 & P-045 & 77 & 1110 \\
\hline P-018 & 78.5 & 1000 & P-046 & 18.9 & 694 \\
\hline P-019 & 58.3 & 874 & P-047 & 49.2 & 1390 \\
\hline P-020 & 140 & 1120 & P-048 & 49.2 & 1450 \\
\hline P-021 & 114 & 982 & P-049 & 117 & 2130 \\
\hline P-022 & 291 & 1490 & P-050 & 116 & 981 \\
\hline P-023 & 7.83 & 326 & P-051 & 331 & 4780 \\
\hline P-024 & 5.63 & 244 & P-052 & 322 & 2080 \\
\hline P-025 & 3.84 & 283 & back sd \#1 & 0.011 & \\
\hline P-026 & 25.0 & 790 & back sd \#2 & 0.015 & \\
\hline P-027 & 45.4 & 758 & & & \\
\hline P-028 & 78.8 & 719 & & & \\
\hline
\end{tabular}




\section{QA/QC ANALYSIS AND RESULTS}

Several QA/QC aspects were built into the analysis procedures and the results are summarized in Table D-4.

- Lanthanum was spiked to each sample from the same solution as the internal standard, In, so that $\mathrm{La}$ and In would always be in the exact same ratio so that an overall instrumental

Table D-4. Summary of QA/QC analyses performed during the course of the analysis of the air filters.

\begin{tabular}{|c|c|c|c|c|c|}
\hline & $\operatorname{Pr}$ & $\mathrm{La}$ & Ho & $\operatorname{Tm}$ & In $(\% \mathrm{Rec})$ \\
\hline $\begin{array}{l}\text { Mean Blank (ng/mL) } \\
(\mathrm{n}=17 \text { calibration } \\
\text { checks) }^{\mathrm{a}}\end{array}$ & $\begin{array}{c}0.001 \\
(0.029)\end{array}$ & $\begin{array}{c}0.006 \\
(0.012)\end{array}$ & $\begin{array}{l}-0.005 \\
(0.011)\end{array}$ & $\begin{array}{l}-0.010 \\
(0.010)\end{array}$ & $\begin{array}{l}104 \\
(4)\end{array}$ \\
\hline $\begin{array}{l}\text { Working Detection } \\
\text { Limit }\left(3^{*} \mathrm{SD}_{\text {blank }}\right)\end{array}$ & 0.088 & 0.036 & 0.032 & 0.029 & \\
\hline $\begin{array}{l}\text { Mean \% Error }(n=20 \\
\text { calibration checks) }^{\mathrm{a}}\end{array}$ & $2.3(3.3)$ & $-1.8(2.7)$ & $-2.9(5.7)$ & $-0.7(5.7)$ & $103(5)$ \\
\hline $\begin{array}{l}\% \text { Recovery of } \\
\text { Surrogates }(n=91 \text { preps } \\
\text { and analyses })^{a}\end{array}$ & & $\begin{array}{l}100.8 \\
(5.5)\end{array}$ & $\begin{array}{l}95.3 \\
(9.8)\end{array}$ & $\begin{array}{c}97 \\
(10)\end{array}$ & $\begin{array}{l}98.3 \\
(5.3)\end{array}$ \\
\hline Blank prep $1(\mathrm{ng} / \mathrm{mL})^{\mathrm{b}}$ & 0.224 & $94.1 \%$ & $107 \%$ & $109 \%$ & 104 \\
\hline Blank prep $2(\mathrm{ng} / \mathrm{mL})^{\mathrm{b}}$ & $<0.088$ & $94.9 \%$ & $110 \%$ & $111 \%$ & 96.1 \\
\hline Blank prep $3(\mathrm{ng} / \mathrm{mL})^{b}$ & $<0.088$ & $97.4 \%$ & $102 \%$ & $103 \%$ & 101 \\
\hline Blank prep $4(\mathrm{ng} / \mathrm{mL})^{\mathrm{b}}$ & 0.154 & $99.6 \%$ & $109 \%$ & $112 \%$ & 98.4 \\
\hline $\begin{array}{l}\text { Blank prep spike } 1 \\
\text { (\% recovery) }\end{array}$ & 114 & 94.1 & 104 & 106 & 93.2 \\
\hline $\begin{array}{l}\text { Blank prep spike } 2^{c} \\
\text { (\% recovery) }\end{array}$ & 126 & 97.4 & 118 & 119 & 94.4 \\
\hline $\begin{array}{l}\text { Blank prep spike } 3^{d} \\
\text { (\% recovery) }\end{array}$ & 188 & 97.9 & 103 & 104 & 97.8 \\
\hline $\begin{array}{l}\text { Blank prep spike } 4^{c} \\
\text { (\% recovery) }\end{array}$ & 103 & 99.6 & 107 & 111 & 99.7 \\
\hline $\begin{array}{l}\text { Blank prep spike } 5 \\
\text { (\% recovery) }\end{array}$ & 100 & 99.4 & 96.0 & 95.6 & 95.6 \\
\hline
\end{tabular}

a. Values in parentheses are the standard deviations.

b. La, Ho, and $\mathrm{Tm}$ expressed as \% recovery.

c. Over recovery of Ho and Tm implies that a similar positive bias exists for Pr.

d. Examination of the results implies that the sample was actually spiked with $2 \mathrm{Pr}$ aliquots and if so, the recovery is $94 \%$. 
analysis precision could be determined that is applicable to each and every sample. The result indicates that over the course of all the analyses the standard deviation will be $-5.5 \%$.

- The calibration check standard was prepared for a commercial mixed element standard (MISA 5 from PlasmaChem) at $100 \mu \mathrm{g} / \mathrm{mL}$ and the calibration standards were prepared from commercial $1000 \mu \mathrm{g} / \mathrm{mL}$ solutions of the individual analytes. This allows an overall determination of accuracy from an independent reference standard. This reference standard was analyzed after every 6 samples and one calibration blank, i.e. every seventh sample in the queue. The calibration checks using the independent standard was performed a total 19 times and a calibration standard was reanalyzed one time with a resulting overall \% error for $\mathrm{Pr}$ being $2.3 \pm 3.3$, i.e. mean accuracy was within $5 \%$ and the all inclusive accuracy within $10 \%$. Recalibration limits for the autosampler logic were $\pm 10 \%$.

- In order to assure that there was a not significant sample-to-sample carry over or low end drift, a calibration blank was included as every seventh sample in the queue for a total of 19 analyses. Two of these results exhibited $\sim 0.2 \mathrm{ng} / \mathrm{mL}$ carry over and were dropped from the resulting averages. The carry over of $0.2 \mathrm{ng} / \mathrm{mL}$ was not of great concern since, at most, any sample bias caused by carry over at this level would have been minimal $(-5 \%$ in the worst case for samples preparation at $\sim 4 \mathrm{ng} / \mathrm{mL}$ ). In actuality, most samples contained $\mathrm{Pr}$ at levels well in excess of $20 \mathrm{ng} / \mathrm{mL}$ and many had to be additionally diluted to fit into the calibration range which only extended to $300 \mathrm{ng} / \mathrm{mL}$. The remaining 17 calibration blank analyses were used to determine if there was a significant offset and a "working" detection limit of $0.088 \mathrm{ng} / \mathrm{mL}$ which is representative of the entire time frame of the actual instrumental analysis.

- To assess the sample preparation procedures and transfers, Ho and Tm were spiked to every sample prior to digestion. Recoveries of Ho and Tm that were significantly low or high would indicate problems that could be associated with sample preparation, transfers or inaccurate spiking of the internal standard solution. For all sample preparations, the mean recoveries of Ho and $\mathrm{Tm}$ were $95 \pm 10$ and $97 \pm 10$, respectively. Recoveries ranged from $76 \%$ for filter sample P-011 to $124 \%$ for reagent blank spike 2. A second dilution of filter sample P-011 confirmed the low recovery of Ho and Tm.

- Reagent blanks and reagent blank spikes were prepared analogously to the actual filter samples except that no filter was present. The results of the individual analyses of these preparations are shown in Table D-4. The first three reagent blank spikes cause some concern because of the over recovery. Reagent blank spike 3 appears to have been spiked with two aliquots of the $10 \mu \mathrm{g} \mathrm{Pr} / \mathrm{mL}$ spiking solution. Reagent blank 2 appears to have been under spiked with the internal standard during the additional sample dilution. A second dilution of reagent blank 2 was not prepared to confirm this. 


\section{Attachment 1}

Pages 1-6 Sample by sample analysis run log and subsequent data reductions for analyses performed on 10/24/94.

Pages 7-11 Sample by sample analysis run log and subsequent data reductions for analyses performed on 10/25/94.

Pages 12-16 Combined summary of all analyses from 10/24/95 and 10/25/95. 



\begin{tabular}{|c|c|c|c|c|c|c|c|c|c|c|c|c|c|}
\hline & & Volu & La 129 & Pr 141 & Ho 165 & $\operatorname{Tm} 169$ & $\ln 115$ & La 12 & Pr 141 & $\operatorname{Pr} 141$ & \multirow{2}{*}{\multicolumn{3}{|c|}{\begin{tabular}{|l|l|} 
Ho 16 & Tm 169 \\
$\%$ Re & $\% \operatorname{Rec}$ \\
\end{tabular}}} \\
\hline ICP-MS Run Log & Mass & $\mathrm{mL}$ & $\mathrm{ng} / \mathrm{mL}$ & $\mathrm{ng} / \mathrm{mL}$ & $\mathrm{ng} / \mathrm{mL}$ & $\mathrm{ng} / \mathrm{mL}$ & $\% \operatorname{Rec}$ & $\% \operatorname{Re}$ & $\mu \mathrm{g} /$ Filter & $\mu g / g$ & & & \\
\hline \multicolumn{14}{|c|}{ Sample Analysis - Filter Blanks } \\
\hline Filter Blk \#1 & 0.0004 & 250 & 96.840 & 0.405 & 42.250 & 43.580 & 107.100 & 96.8 & 0.101 & & 84.5 & 87.2 & \\
\hline Filter Blk \#1 rpt & 0.0004 & 250 & 101.100 & 0.431 & 43.490 & 44.250 & 93.360 & 101.1 & 0.108 & & 87.0 & 88.5 & \\
\hline Filter Blk \#2 & 0.0004 & 250 & 100.200 & 0.394 & 43.950 & 45.180 & 95.980 & 100.2 & 0.098 & & 87.9 & 90.4 & \\
\hline Filter BIk \#3 & 0.0005 & 250 & 96.760 & 0.398 & 44.940 & 45.810 & 104.200 & 96.8 & 0.099 & & 89.9 & 91.6 & \\
\hline Filter Blk \#3 rpt & 0.0005 & 250 & 101.200 & 0.418 & 44.550 & 46.010 & 95.760 & 101.2 & 0.104 & & 89.1 & 92.0 & \\
\hline Filter Blk \#4 & 0.0010 & 250 & 99.580 & 0.455 & 46.330 & 47.480 & 95.770 & 99.6 & $\overline{0.114}$ & & 92.7 & 95.0 & \\
\hline Filter Blk \#5 & -0.0001 & 250 & 99.740 & 0.411 & 41.850 & 43.010 & 98.200 & 99.7 & 0.103 & & 83.7 & 86.0 & \\
\hline Filter Blk \#6 & -0.0002 & 250 & 100.600 & 0.453 & 45.240 & 46.280 & 97.400 & 100.6 & 0.113 & & 90.5 & 92.6 & \\
\hline \multirow[t]{5}{*}{ Filter Blk \#8 } & -0.0006 & 250 & 101.200 & 0.442 & 47.070 & 48.460 & 97.890 & 101.2 & 0.110 & & 94.1 & 96.9 & \\
\hline & & & & & & & & & & & & & \\
\hline & & & & & \multicolumn{2}{|c|}{ Mean Filter Blank } & & & 0.106 & & & & \\
\hline & & & & & SD & & & & 0.006 & & & & \\
\hline & & & & & & & & & & & & & \\
\hline Sample Summary & & & & & & & & \multicolumn{6}{|c|}{ Pr data corrected for mean Filter blank } \\
\hline Back Side \#1 & & 250 & 101.300 & 0.468 & 41.250 & 41.480 & 95.700 & 101.3 & 0.011 & & 82.5 & 83.0 & Estimate $2 \mathrm{mg}$ Dus \\
\hline Back Side \#2 & & 250 & 101.300 & 0.485 & 43.680 & 43.660 & 95.080 & 101.3 & 0.015 & & 87.4 & 87.3 & Estimate $3 \mathrm{mg}$ Dus \\
\hline Filter\#1 & 0.0097 & 250 & 93.900 & 2.599 & 50.800 & 52.130 & 103.600 & 93.9 & 0.650 & 67.0 & 101.6 & 104.3 & \\
\hline Filter \#2 & 0.0309 & 250 & 94.140 & 7.052 & 50.980 & 52.460 & 100.100 & 94.1 & 1.763 & 57.1 & 102.0 & 104.9 & \\
\hline Filter \#3 & 0.0099 & 250 & 93.960 & 1.821 & 50.490 & 51.550 & 98.870 & 94.0 & 0.455 & 46.0 & 101.0 & 103.1 & \\
\hline Filter \#4 & 0.0575 & 250 & 97.200 & 341.800 & 51.990 & 53.210 & 96.590 & 97.2 & 85.450 & 1486.1 & 104.0 & 106.4 & Dilute to rerun \\
\hline Filter \#4 1:5 & 0.0575 & 1250 & 99.260 & 66.428 & 9.351 & 9.448 & 101.600 & 99.3 & 82.929 & 1442.2 & 93.5 & 94.5 & Diluted \\
\hline Filter \#5 & 0.1352 & 250 & 99.880 & 381.800 & 44.850 & 45.160 & 97.180 & 99.9 & 95.450 & 706.0 & 89.7 & 90.3 & Dilute to rerun \\
\hline Filter \#5 1:5 & 0.1352 & 1250 & 100.700 & 77.918 & 9.062 & 9.408 & 105.500 & 100.7 & 97.292 & 719.6 & 90.6 & 94.1 & Diluted \\
\hline Filter \#6 & 0.1216 & 250 & 99.280 & 362.800 & 44.170 & 44.470 & 99.850 & 99.3 & 90.700 & 745.9 & 88.3 & 88.9 & Dilute to rerun \\
\hline Filter\#6 1:5 & 0.1216 & 1250 & 98.990 & 71.648 & 8.596 & 8.756 & 104.700 & 99.0 & 89.454 & 735.6 & 86.0 & 87.6 & Diluted \\
\hline Filter \#7 & 0.1982 & 250 & 104.100 & 921.600 & 41.500 & 41.490 & 97.760 & 104.1 & 230.400 & 1162.5 & 83.0 & 83.0 & Dilute to rerun \\
\hline Filter \#8 & 0.0172 & 250 & 96.160 & 24.510 & 47.210 & 47.390 & 95.970 & 96.2 & 6.128 & 356.3 & 94.4 & 94.8 & \\
\hline Filter \#9 & 0.0226 & 250 & 95.350 & 36.570 & 47.080 & 47.690 & 97.260 & 95.4 & $\overline{9.143}$ & 404.5 & 94.2 & 95.4 & \\
\hline Filter \#10 & 0.1769 & 250 & 117.400 & 760.128 & 48.520 & 49.260 & 90.500 & 117.4 & 189.926 & 1073.6 & 97.0 & 98.5 & \\
\hline Filter \#11 & 0.5029 & 250 & 128.700 & 1824.000 & 38.720 & 38.390 & 91.960 & 128.7 & 456.000 & 906.7 & 77.4 & 76.8 & Diluted to rerun \\
\hline Filter \#11 1:10 & 0.5029 & 2500 & 100.200 & 174.828 & 3.821 & 3.806 & 107.800 & 100.2 & 436.964 & 868.9 & 76.4 & 76.1 & Diluted \\
\hline Filter \#12 & 0.0130 & 250 & 96.220 & 18.200 & 45.810 & 46.340 & 97.210 & 96.2 & 4.550 & 350.0 & 91.6 & 92.7 & \\
\hline Filter \#13 & 0.0097 & 250 & 95.580 & 2.463 & 47.420 & 47.640 & 95.030 & 95.6 & 0.616 & 63.5 & 94.8 & 95.3 & \\
\hline Filter \#14 & 0.0058 & 250 & 94.630 & 1.242 & 49.950 & 50.750 & 93.560 & 94.6 & 0.311 & 53.5 & 99.9 & 101.5 & \\
\hline
\end{tabular}

Attachment 1 Page 1 


\begin{tabular}{|c|c|c|c|c|c|c|c|c|c|c|c|c|c|}
\hline & & Volu & La 129 & Pr 141 & Ho 165 & $\operatorname{Tm} 169$ & $\ln 115$ & La 12 & $\operatorname{Pr} 141$ & $\operatorname{Pr} 141$ & Ho 1 & $\operatorname{Tm} 16$ & \\
\hline ICP-MS Run Log & Mass & $\mathrm{mL}$ & $\mathrm{ng} / \mathrm{mL}$ & $n g / m L$ & $n g / m L$ & $\mathrm{ng} / \mathrm{mL}$ & $\% \operatorname{Rec}$ & $\% \operatorname{Re}$ & $\mu \mathrm{g} /$ Filter & $\mu g / g$ & $\% \operatorname{Re}$ & $\% \operatorname{Rec}$ & \\
\hline Filter \#15 & 0.0590 & 250 & 101.400 & 394.000 & 57.740 & 58.270 & 90.800 & 101.4 & 98.500 & 1669.5 & 115.5 & 116.5 & \\
\hline Filter \#16 & 0.0389 & 250 & 98.300 & 160.200 & 53.570 & 54.510 & $\overline{92.010}$ & 98.3 & 40.050 & 1029.6 & 107.1 & 109.0 & \\
\hline Filter \#17 & 0.0936 & 250 & 102.500 & 411.400 & 54.510 & 54.940 & 88.910 & 102.5 & 102.850 & 1098.8 & 109.0 & 109.9 & \\
\hline Filter \#18 & 0.0784 & 250 & 102.500 & 313.900 & 53.170 & 53.480 & 92.640 & 102.5 & 78.475 & 1001.0 & 106.3 & 107.0 & \\
\hline Filter \#19 & 0.0667 & 250 & 99.720 & 233.100 & 48.470 & 48.930 & 96.150 & 99.7 & 58.275 & 873.7 & 96.9 & 97.9 & \\
\hline Filter \#20 & 0.1247 & 250 & 105.100 & 577.300 & 50.170 & 50.440 & 94.030 & 105.1 & 144.325 & 1157.4 & 100.3 & 100.9 & Diluted to rerun \\
\hline Filter \#20 1:5 & 0.1247 & 1250 & 99.740 & 108.328 & 9.635 & 9.682 & 107.800 & 99.7 & 135.304 & 1085.0 & 96.4 & 96.8 & Diluted \\
\hline Filter \#21 & 0.1164 & 250 & 104.200 & 470.500 & 49.280 & 49.290 & 90.590 & 104.2 & 117.625 & 1010.5 & 98.6 & 98.6 & Dilute to rerun \\
\hline Filter $\$ 211: 5$ & 0.1164 & 1250 & 99.180 & 88.908 & 9.815 & 9.807 & 107.100 & 99.2 & 111.029 & 953.9 & 98.2 & 98.1 & Diluted \\
\hline Filter \#22 & 0.1951 & 250 & 113.000 & 1198.000 & 49.770 & 49.900 & 92.010 & 113.0 & 299.500 & 1535.1 & 99.5 & 99.8 & Dilute to rerun \\
\hline Filter \#22 1:5 & 0.1951 & 1250 & 101.600 & 226.828 & 10.260 & 10.260 & 105.600 & 101.6 & 283.429 & 1452.7 & 102.6 & 102.6 & Diluted \\
\hline Filter \#23 & 0.0240 & 250 & 95.830 & 31.320 & 44.720 & 45.220 & 96.790 & 95.8 & 7.830 & 326.3 & 89.4 & 90.4 & \\
\hline Filter \#24 & 0.0231 & 250 & 94.410 & 22.500 & 44.950 & 45.440 & 101.500 & 94.4 & 5.625 & 243.5 & 89.9 & 90.9 & \\
\hline Filter \#25 & 0.0136 & 250 & 93.860 & 15.460 & 53.350 & 53.660 & 84.470 & 93.9 & 3.865 & 284.2 & 106.7 & 107.3 & \\
\hline Filter \#25 rpt & 0.0136 & 250 & 99.760 & 15.290 & 56.630 & 57.840 & 93.200 & 99.8 & 3.823 & 281.1 & 113.3 & 115.7 & \\
\hline Filter \#26 & 0.0317 & 250 & 95.020 & 99.930 & 47.160 & 47.870 & 93.010 & 95.0 & 24.983 & 788.1 & 94.3 & 95.7 & \\
\hline Filter \#26 rpt & 0.0317 & 250 & 101.700 & 100.400 & 53.860 & 54.910 & 96.150 & \begin{tabular}{|l|}
101.7 \\
\end{tabular} & 25.100 & 791.8 & 107.7 & 109.8 & \\
\hline Filter \#27 & 0.0599 & 250 & 103.500 & 181.700 & 46.400 & 47.290 & 96.990 & 103.5 & 45.425 & 758.3 & 92.8 & 94.6 & \\
\hline Filter \#28 & 0.1096 & 250 & 113.400 & 315.000 & 54.400 & 56.370 & 91.030 & 113.4 & 78.750 & 718.5 & 108.8 & 112.7 & \\
\hline Filter \#29 & 0.0802 & 250 & 106.000 & 173.200 & 46.580 & 47.410 & 97.620 & 106.0 & 43.300 & 539.9 & 93.2 & 94.8 & \\
\hline Filter \#30 & 0.0540 & 250 & 107.700 & 101.000 & 55.340 & 58.030 & 91.510 & \begin{tabular}{|l|}
107.7 \\
\end{tabular} & 25.250 & 467.6 & 110.7 & 116.1 & \\
\hline Filter \#31 & 0.0830 & 250 & 112.300 & 207.200 & 57.450 & 60.680 & 90.390 & $\mid$\begin{tabular}{|l|}
112.3 \\
\end{tabular} & 51.800 & 624.1 & 114.9 & 121.4 & \\
\hline Filter \#32 & 0.0528 & 250 & 105.500 & 142.800 & 52.260 & 55.160 & 95.690 & \begin{tabular}{|l|}
105.5 \\
\end{tabular} & 35.700 & 676.1 & 104.5 & 110.3 & \\
\hline Filter \#33 & 0.0503 & 250 & 102.100 & 185.200 & 43.300 & 44.070 & 97.450 & 102.1 & 46.300 & 920.5 & 86.6 & 88.1 & \\
\hline Filter \#34 & 0.0188 & 250 & 100.300 & 52.230 & 47.610 & 48.270 & 99.300 & \begin{tabular}{|l|}
100.3 \\
\end{tabular} & 13.058 & 694.5 & 95.2 & 96.5 & \\
\hline Filter \#35 & 0.0501 & 250 & 103.600 & 213.500 & 47.180 & 48.300 & 94.310 & 103.6 & 53.375 & 1065.4 & 94.4 & 96.6 & \\
\hline Filter \#36 & 0.0945 & 250 & 105.800 & 383.900 & 41.450 & 42.050 & 97.950 & 105.8 & 95.975 & 1015.6 & 82.9 & 84.1 & Dilute to rerun \\
\hline Filter \#36 1:5 & 0.0945 & 1250 & 99.190 & 78.998 & 8.083 & 8.071 & 105.900 & 99.2 & 98.642 & 1043.8 & 80.8 & 80.7 & Diluted \\
\hline Filter \#37 & 0.0956 & 250 & \begin{tabular}{|l|}
107.200 \\
\end{tabular} & 328.600 & 45.900 & 46.400 & 99.240 & \begin{tabular}{|l|}
107.2 \\
\end{tabular} & 82.150 & 859.3 & 91.8 & 92.8 & \\
\hline Filter \#38 & 0.0282 & 250 & 99.680 & 67.580 & 43.620 & 44.600 & 101.800 & 99.7 & 16.895 & 599.1 & 87.2 & 89.2 & \\
\hline Filter \#39 & 0.0680 & 250 & 103.100 & 250.800 & 46.060 & 46.910 & 101.100 & 103.1 & 62.700 & 922.1 & 92.1 & 93.8 & \\
\hline Filter \#40 & 0.0368 & 250 & 100.600 & 160.400 & 46.850 & 46.920 & 100.100 & 100.6 & 40.100 & 1089.7 & 93.7 & 93.8 & \\
\hline Filter \#41 & 0.0504 & 250 & 104.6 & 254 & 50.14 & 51.53 & 98.840 & 104.6 & 63.500 & 1259.9 & 100.3 & 103.1 & \\
\hline Filter \#42 & 0.0095 & 250 & 95.770 & 5.626 & 41.530 & 42.460 & 105.000 & 95.8 & 1.407 & 148.1 & 83.1 & 84.9 & \\
\hline Filter \#43 & 0.0096 & 250 & 98.970 & 3.915 & 46.410 & 47.820 & 100.900 & 99.0 & 0.979 & 102.0 & 92.8 & 95.6 & \\
\hline Filter \#44 & 0.0093 & 250 & 99.280 & 4.376 & 47.620 & 49.670 & 101.300 & 99.3 & 1.094 & 117.6 & 95.2 & 99.3 & \\
\hline
\end{tabular}

Attachment 1 Page 2 


\begin{tabular}{|c|c|c|c|c|c|c|c|c|c|c|c|c|c|}
\hline & & Volu & La 129 & $\operatorname{Pr} 141$ & Ho 165 & Tm 169 & $\ln 115$ & La 12 & Pr 141 & Pr 141 & Ho 16 & $\operatorname{Tm} 169$ & \\
\hline ICP-MS Run Log & Mass & $\mathrm{mL}$ & Ing/mL & $\mathrm{ng} / \mathrm{mL}$ & $n g / m L$ & $n g / m L$ & \% Rec & $\% \operatorname{Re}$ & $\mu \mathrm{g} /$ Filter & $\mu g / g$ & $\% \operatorname{Re} \mid$ & $\% \operatorname{Rec}$ & - \\
\hline Filter \#45 & 0.0695 & 250 & 102.800 & 308.000 & 44.460 & 45.190 & 99.000 & 102.8 & 77.000 & 1107.9 & 88.9 & 90.4 & \\
\hline Filter \#46 & 0.0272 & 250 & 102.600 & 75.918 & 41.510 & 42.020 & 95.010 & 102.6 & 18.874 & 693.9 & 83.0 & 84.0 & \\
\hline Filter \#47 & 0.0353 & 250 & 100.900 & 196.700 & 46.170 & 46.670 & 109.200 & 100.9 & 49.175 & 1393.1 & 92.3 & 93.3 & \\
\hline Filter \#48 & 0.0339 & 250 & 100.600 & 196.700 & 46.310 & 47.280 & 108.100 & 100.6 & 49.175 & 1450.6 & 92.6 & 94.6 & \\
\hline Filter \#49 & 0.0550 & 250 & 102.800 & 472.100 & 43.760 & 44.470 & 100.400 & 102.8 & 118.025 & 2145.9 & 87.5 & 88.9 & Dilute to rerun \\
\hline Filter\#49 1:5 & 0.0550 & 1250 & 97.860 & 92.808 & 7.934 & 7.942 & 104.100 & 97.9 & 115.904 & \begin{tabular}{|l|}
2107.3 \\
\end{tabular} & 79.3 & 79.4[ & Diluted \\
\hline Filter \#50 & 0.1185 & 250 & 101.900 & 461.700 & 44.230 & 44.920 & 104.200 & 101.9 & 115.425 & 974.1 & 88.5 & 89.8 & Dilute to rerun \\
\hline Filter \#50 1:5 & 0.1185 & 1250 & 98.380 & 93.738 & 8.092 & 8.074 & 106.100 & 98.4 & \begin{tabular}{|l|}
117.067 \\
\end{tabular} & 987.9 & 80.9 & 80.7 & Diluted \\
\hline Filter $\# 51$ & 0.0691 & 250 & 105.500 & 1287.000 & 43.010 & 43.350 & 103.800 & 105.5 & 321.750 & 4656.3 & 86.0 & 86.7 & Dilute to rerun \\
\hline Filter \#51 1:10 & 0.0691 & 2500 & 96.690 & 128.828 & 3.953 & 3.976 & 108.800 & 96.7 & 321.964 & 4659.4 & 79.1 & 79.5 & Diluted \\
\hline Filter \#51 1:10 rot & 0.0691 & 2500 & 102.200 & 139.228 & 4.557 & 4.607 & 98.640 & 102.2 & 347.964 & $5035.7 \mid$ & 91.1 & 92.1 & Diluted \\
\hline Filter $\# 52$ & 0.1548 & 250 & 111.900 & 1263.000 & 46.770 & 47.090 & 103.200 & 111.9 & 315.750 & 2039.7 & 93.5 & 94.2 & Dilute to rerun \\
\hline Filter \#52 1:10 & 0.1548 & 2500 & 97.330 & 125.728 & 4.184 & 4.219 & 108.200 & 97.3 & 314.214 & 2029.8 & 83.7 & 84.4 & Diluted \\
\hline Filter \#52 1:10 rpt & 0.1548 & 2500 & 101.300 & 134.628 & 4.926 & 4.878 & 96.260 & 101.3 & 336.464 & 2173.5 & 98.5 & 97.6 & Diluted \\
\hline & & & & & & & & & & & & & \\
\hline Prep Blk \#1 & 1.0000 & 250 & 94.080 & 0.224 & 53.360 & 54.720 & 104.200 & 94.1 & 0.056 & 0.1 & 106.7 & 109.4 & \\
\hline Prep BIk \#2 & 1.0000 & 250 & 94.870 & 0.023 & 55.270 & 55.660 & 96.070 & 94.9 & 0.006 & 0.0 & 110.5 & 111.3 & $\mathrm{Pr}<\mathrm{DL}$ \\
\hline Prep BIk \#3 & 1.0000 & 250 & 97.360 & 0.047 & 51.100 & 51.570 & 101.100 & 97.4 & 0.012 & 0.0 & 102.2 & 103.1 & $\mathrm{Pr}<\mathrm{DL}$ \\
\hline Prep Blk \#4 & 1.0000 & 250 & 99.630 & 0.154 & 54.370 & 56.090 & 98.390 & 99.6 & 0.039 & 0.0 & 108.7 & 112.2 & \\
\hline Prep Blk Spk \#1 & 1.0000 & 250 & 93.130 & 37.080 & 53.530 & 55.350 & 96.960 & 93.1 & 9.270 & 9.3 & 107.1 & 110.7 & \\
\hline Prep Blk Spk \#2 & 1.0000 & 250 & 95.010 & 40.540 & 56.130 & 57.020 & 93.780 & 95.0 & 10.135 & 10.1 & 112.3 & \begin{tabular}{|l|}
114.0 \\
\end{tabular} & \\
\hline Prep Blk Spk \#3 ?? & 1.0000 & 250 & 96.860 & 58.880 & 50.610 & 51.770 & 101.300 & 96.9 & 14.720 & 14.7 & 101.2 & 103.5 & Spiked twice? \\
\hline Prep Blk Spk \#4 & 1.0000 & 250 & 99.640 & 32.930 & 53.600 & 55.490 & 99.670 & 99.6 & 8.233 & 8.2 & 107.2 & 111.0 & Ho, Tm also high? \\
\hline Prep Blank Spike \#5 & 1.0000 & 250 & 99.430 & 32.048 & 47.990 & 47.890 & 95.870 & 99.4 & 8.012 & 8.0 & 96.0 & 95.8 & \\
\hline Prep Blank Spike (2) \#1 & 1.0000 & 250 & 95.160 & 36.338 & 50.580 & 50.970 & 89.470 & 95.2 & 9.084 & 9.1 & 101.2 & 101.9 & \\
\hline Prep Blank Spike (2) \#2 & 1.0000 & 250 & 99.770 & 40.118 & 61.860 & 62.180 & 91.770 & 99.8 & 10.029 & 10.0 & 123.7 & 124.4 & Ho, Tm also high \\
\hline Prep Blank Spike (2) \#3 & 1.0000 & 250 & 98.910 & 61.568 & 52.000 & 52.740 & 94.290 & 98.9 & 15.392 & 15.4 & 104.0 & 105.5 & Spiked Twice? \\
\hline & \multicolumn{2}{|c|}{ Expected } & & 32.000 & & & & & 8.000 & 8.0 & & & \\
\hline & & & & & & & & & & & & & \\
\hline Mean \% Recovery & & & & & & & 98.3 & 100.8 & & & 95.3 & 96.8 & \\
\hline \multicolumn{2}{|c|}{ standard deviation } & & & & & & 5.3 & 5.5 & & & 9.8 & 10.4 & \\
\hline Count & & & & & & & 91 & 91 & & & 91 & 91 & \\
\hline \multicolumn{6}{|c|}{ standard error of the mean - sd/sgrt(n) } & & 0.6 & 0.6 & & & 1.0 & 1.1 & \\
\hline \multicolumn{5}{|l|}{ t*sd/sqrt (n) } & $-1 . e$ & . conf & 1.1 & 1.1 & & & 2.0 & 2.2 & \\
\hline t*sd $(95 \% \mathrm{CL})-$ & i.e. & rang & le of ex & xpected & values & s at 9 & 10.5 & 10.9 & & & 19.4 & 20.6 & \\
\hline
\end{tabular}

Attachment 1 Page 3 


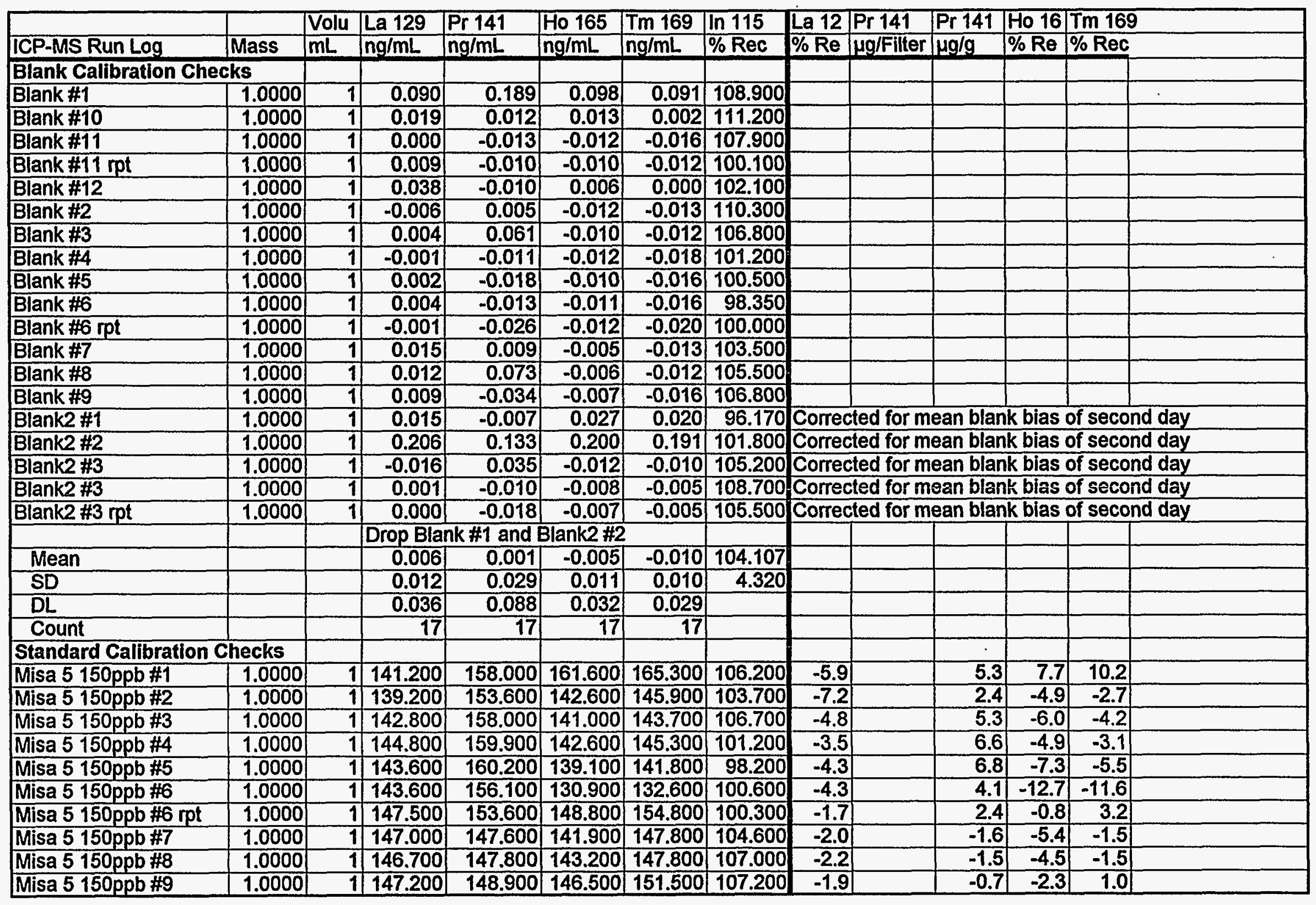

Attachment 1 Page 4 
Combined summary of all analyses for Pr on glass fiber air filters

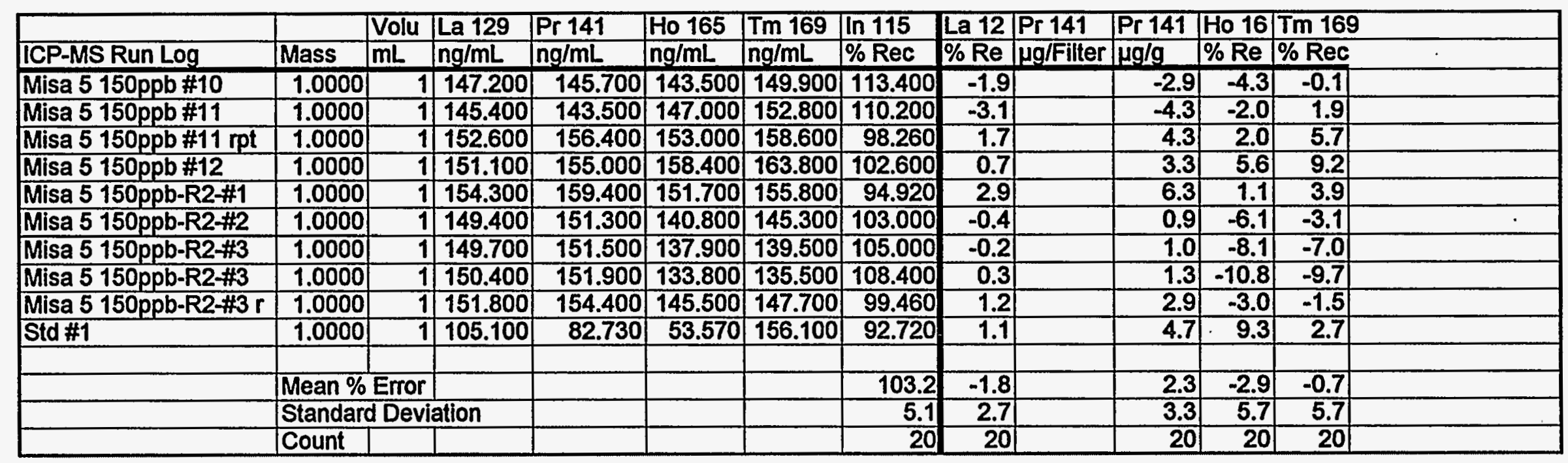

Attachment 1 -

Page 5 


\section{Appendix E}

Corrected Test Procedures 



\section{Appendix E \\ Test Procedure Phase I Demonstration Dust and Contamination Spread}

\section{TEST SET-UP}

1. Construct simulated waste test bed as per RAHCO drawing 2191AA-1201-00-00.

2. Attach end effector and ITM to excavator boom.

3. Position test equipment including excavator, transport container stands, ITMs, and transport containers. The excavator will be positioned near the test bed. The ITMs will be placed 90 degrees from the test bed with respect to the excavator boom rotation.

4. Start excavator and check out all movements and operations.

5. Confirm that all fuel tanks and lubricant levels are full and ready for operations.

6. Close west door of building. Seal any large cracks in building walls and doors.

7. Set up particulate air samplers. The air samplers will be positioned as follows:
a. two will be placed near the simulated waste pile;
b. two will be placed near the excavator chassis; and
c. four will be placed around the ITMs.

8. Position video cameras and lights.

9. Hold Test Readiness Review Meeting. Review test set-up, test procedures, safety issues, etc.

1. Obtain 60 minutes worth of background data from the HI-VOL samplers, then pull the background sample filters and replace with new filters.

2. Start the excavator.

3. Start the HI-VOL samplers (see separate procedures for start-up of the HI-VOL samplers).

4. Start video cameras. 
5. Begin excavating test bed per sequence shown on RAHCO Drawing 2191AA-1201-00-00 and place full ITM on to the floor.

6. Record volume of waste in ITM and spillage during transfer and record excavating/transfer time. Also, note any deviations from normal operating sequences.

7. Excavator deposits ITM onto the floor.

8. Excavator mates end effector with empty ITM, attaches, and lifts ITM up from floor.

9. Forklift enters building from south door of building.

10. Forklift transports ITM through south door of building.

11. Forklift empties ITM at designated location outside of building.

12. Wipe excess dust from ITM.

13. Forklift returns empty ITM near excavator.

14. Repeat steps 6 through 13 until $10 \mathrm{yd}^{3}$ of waste has been removed (or approximately 1 to 2 hours of testing have been completed).

15. Remove air sample filters.

16. Repeat steps 1 through 18 each new day until test bed is completely excavated (approximately 4 days).

NOTE: For removal of large items from test bed, follow steps 6 through 16 without the use of the ITM. 


\section{Appendix E \\ Test Procedure \\ Phase II Demonstration Retrieval and Conveyance}

\section{TEST SET-UP}

1. Construct simulated hill as per RAHCO drawing 2191BA-1200-00-00.

2. Construct simulated waste test bed. Note, this is built as the Phase I Demonstration and is performed by simply dumping the excavated waste into a pile at the designated location.

3. Attach end effector and ITM to excavator boom.

4. Position test equipment including excavator, TTV, ITMs, transport containers, transport container stands, and remote operations control trailer. The excavator will be positioned near the test bed. The TTV will be placed 180 degrees from the test bed with respect to the excavator boom rotation. A waste container and an ITM will be positioned on the TTV. The transport container stand will be positioned at the end of the TTV path. The other waste container and ITMs will also be placed at the end of the TTV path. The remote operations control trailer will be positioned as per RAHCO drawing 2191BA-1200-00-00.

5. Attach all TTV, excavator, and receiving station reference marks.

6. Position TTV path marking posts.

7. Start the generator for the remote operations control trailer and verify power to operate control station.

8. Start the excavator and check out all movements and operations.

9. Confirm the all fuel tanks and lubricant levels are full and ready for operation.

10. Position video cameras and lights.

11. Hold Test Readiness Review meeting. Review test set-up, test procedures, safety issues, etc.

TEST

1. Start the remote operations control trailer generator.

2. Start video cameras.

3. Start excavator. 
4. Begin excavating portion of Phase II testing.

5. Begin excavating test bed and place full ITM into transport container on TTV. Record time for - placement, release, and reattachment of ITMs.

6. Record volume of waste in ITM and spillage during transfer.

7. TTV lowers container lid.

8. TTV advances 9 feet.

9. Take time for TTV location measurements.

10. Excavator mates end effector with empty ITM, attaches, and lifts empty ITM up from TTV.

11. Note, while TTV is gone from digface, the excavator fills the empty ITM on the end effector with waste.

12. TTV travels to receiving station.

13. TTV docks at receiving station.

14. Take time for TTV location measurements.

15. Load empty ITM.

16. Open lid and remove loaded ITM on TTV using forklift.

17. Close lid.

18. Back TTV away from receiving station.

19. TTV travels to digface.

20. Note, while TTV is traveling and parking at digface the forklift empties the ITM.

21. TTV parks at digface.

22. TTV raises lid.

23. Take time for TTV location measurements.

24. Repeat steps 13 through 36 until $80 \mathrm{yd}^{3}$ of waste has been excavated (or end of day). Repeat steps 13 through 36 each new day until $400 \mathrm{yd}^{3}$ of waste has been excavated (approximately 4 days). 


\section{Appendix E \\ Test Procedure \\ Background Tracer Measurement}

NOTES

A. The test should be performed in the building to be used during the dust test. The area should be free of equipment use or dust generating activities for 24 hours before the start of this test.

B. Although it is not industry standard, the manometers were calibrated using readings taken from the top of the meniscus. For consistency, all manometer readings should be taken from the top of the meniscus.

C. Caution: Always handle the paper filters with tweezers. Do not touch the filters with fingers.

\section{TEST PROCEDURE}

1. Note filter number in logbook.

2. Open cover (lid) of HI-VOL air monitor, and unscrew/open filter holder cover.

3. Using tweezers, remove filter from petri dish and place on HI-VOL filter holder. Replace the lid on the empty petri dish.

4. Replace filter holder cover and close HI-VOL lid.

5. Turn on power to HI-VOL.

6. Note start time, barometric pressure, temperature, and manometer reading in logbook.

7. Note manometer reading, barometric pressure, temperature, time, and level of dust (visual) on filter every 30 minutes for the first 2 hours of testing. Thereafter, take these readings every hour.

8. Take a final reading of manometer, barometric pressure, temperature, time, and level of dust (visual) after 8 hours of operation.

9. Turn off power to HI-VOL.

10. Using tweezers, remove the filter and replace it in the petri dish. Seal the petri dish using tape.

11. Repeat steps 1 through 10 two times, for a total of two background tests.

12. Send photocopies of logbook entries and filter in petri dish to Ann Marie Smith at the INEL.

13. Store HI-VOL at RAHCO until demonstration begins. 


\section{Appendix E \\ Test Procedure \\ HI-VOL Air Monitors}

\section{NQTES}

A. The area should be free of equipment use or dust generating activities for 24 hours before the start of this test.

B. Although it is not industry standard, the manometers were calibrated using readings taken from the top of the meniscus. For consistency, all manometer readings should be taken from the top of the meniscus.

C. Caution: Always handle the paper filters with tweezers. Do not touch the filters with fingers.

D. The filter must be oriented properly in the air monitor. The correct orientation is checkerboard (rough) side down, fuzzy side up.

\section{TEST PROCEDURE}

1. Note filter number and weight in logbook.

2. Open cover (lid) of HI-VOL air monitor, and unscrew/open filter holder cover.

3. Using tweezers, remove filter from petri dish and place on HI-VOL filter holder. The filter must be oriented properly in the air monitor. The correct orientation is checkerboard (rough) side down, fuzzy side up. Replace the lid on the empty petri dish.

4. Replace filter holder cover and close HI-VOL lid.

5. Zero the manometer so that with power off, the manometer reads zero, and note the manometer reading in the logbook.

6. Turn on power to HI-VOL.

7. Note start time, barometric pressure, temperature, and manometer reading in logbook.

8. Note manometer reading, barometric pressure, temperature, and time every 15 minutes for the duration of testing.

9. At the completion of the test, take a final reading of manometer, barometric pressure, temperature, and time.

10. Turn off power to HI-VOL.

11. Using tweezers, remove the filter and replace it in the petri dish. Seal the petri dish using tape, and identify (label) it per the Statement of Work. 


\section{Appendix F}

\section{Dust and Rare Earth Tracer Analysis}





\section{Appendix F}

\section{Test Results for Dust Generation and Contamination Spread}

F-1. DUST GENERATION

\section{Analytical Procedure}

To assess whether the dust detected on the air sample filters exceeds the background level of dust in the air by $1.1 \times 10^{-4} \mathrm{~g} / \mathrm{L}$, a statistical t-test was employed. The procedure tests the hypothesis of equality of the mean dust detected during operations to the background dust plus the target level of $1.1 \times 10^{-4} \mathrm{~g} / \mathrm{L}$ (e.g., is the amount of dust detected on the air sample filters equal to the background level of dust in the air plus $1.1 \times 10^{-4} \mathrm{~g} / \mathrm{L}$ ?)

Null Hypothesis: $\quad H_{o}: \quad$ Mean Dust Detected $\leq$ Background Dust $+1.1 \times 10^{-4} \mathrm{~g} / \mathrm{L}$ or

Null Hypothesis:

$H_{o}: \quad u_{d} \leq u_{b}+1.1 \times 10^{-4}$

Alternate Hypothesis: $\quad H_{1}: \quad$ Mean Dust Detected $>$ Background Dust $+1.1 \times 10^{-4} \mathrm{~g} / \mathrm{L}$ or

Alternate Hypothesis: $\quad H_{1}: \quad u_{d}>u_{b}+1.1 \times 10^{-4}$

where

$$
\begin{aligned}
& u_{d}=\text { the mean of the dust detected on the filters } \\
& u_{b}=\text { the mean of the background dust measurements taken during the test }
\end{aligned}
$$

Because the two means are unknown, a pooled t-test or two-sample t-test will be used in the analysis. The assumption is that both distributions are normal.

$$
\text { The test statistic is therefore: } \quad \cdot t=\frac{\bar{x}_{d}-\left(\bar{x}_{b}+1.1 \times 10^{-4}\right)}{\sqrt{S_{d}^{2} / n_{d}+S_{b}^{2} / n_{b}}}
$$

where

$$
\begin{aligned}
& t=\text { the critical value } \\
& \bar{x}_{d}=\text { the observed mean of the dust detected } \\
& \bar{x}_{b}=\text { the observed mean of the background dust detected } \\
& n_{d} \quad=\quad \text { the number of test filters } \\
& n_{b} \quad=\quad \text { the number of background filters }
\end{aligned}
$$




$$
\begin{aligned}
& S_{d}^{2}=\text { the variance of the dust detected } \\
& S_{b}^{2}=\text { the variance of the background dust }
\end{aligned}
$$

The null hypothesis $\left(H_{o}\right)$ is not rejected when:

$$
t<t\left(\alpha, n_{d}+n_{b}-2\right)
$$

where

$$
\alpha \quad=\quad \text { level of significance }
$$

The null hypothesis $\left(H_{O}\right)$ is rejected in favor of the alternate hypothesis $\left(H_{l}\right)$ when:

$$
t>t\left(\alpha, n_{d}+n_{b}-2\right)
$$

Data Quality Objective: Determine with a $95 \%$ confidence level if dust at $1.1 \times 10^{-4} \mathrm{~g} / \mathrm{L}$ of air above background levels was detected.

Test Data Analysis: Figures F-1 through F-5 show graphs of the dust measured for the test backgrounds and test samples. The raw data, calculation of the mean and standard deviation, and the t-test analysis for each test are presented in Tables F-1 through F-5. 
Dust Measurement - Test \#1

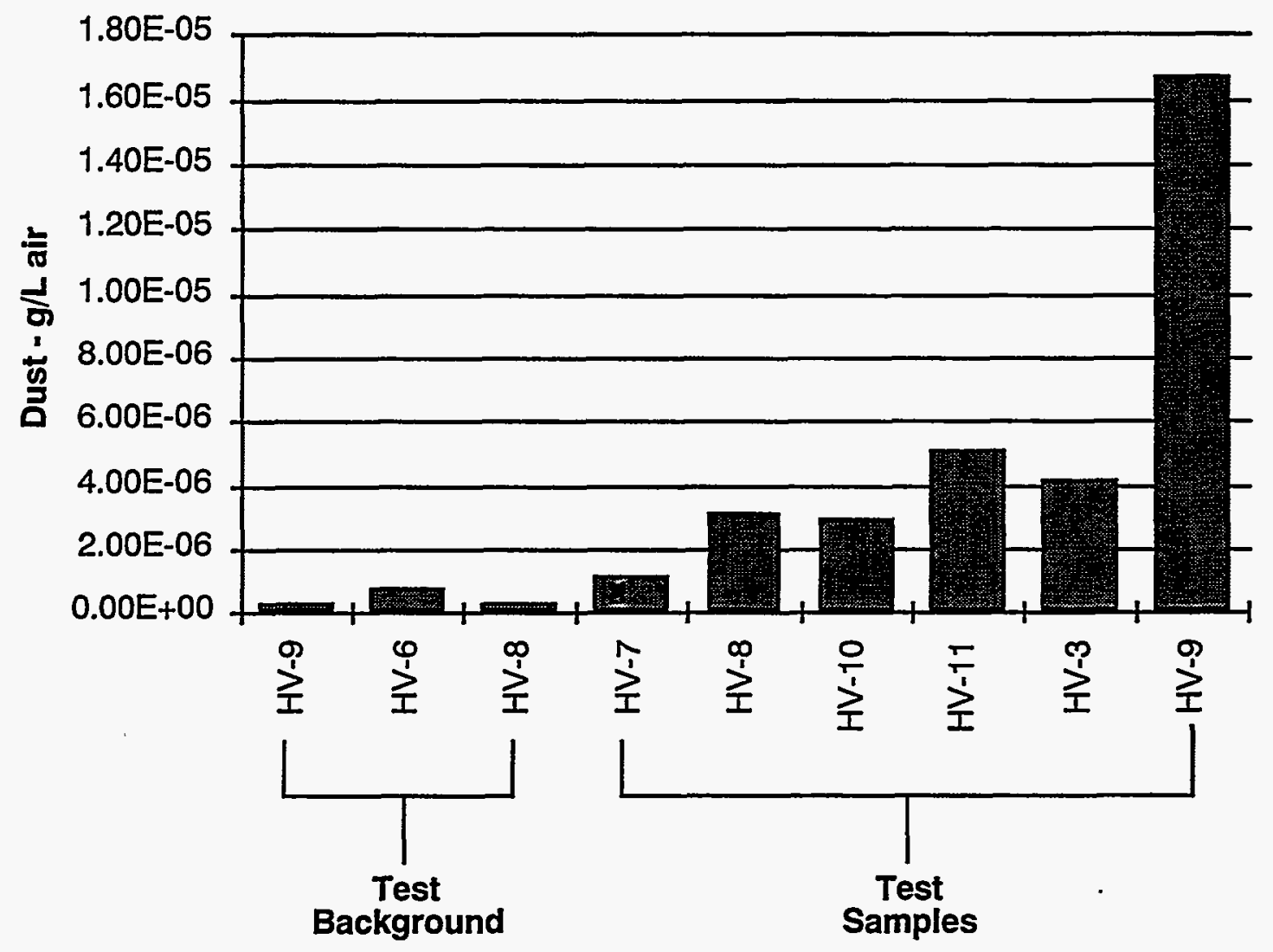

Air Sampler

Figure F-1. The dust measurement graph for test 1 'shows the test sample levels were less then $1.1 \times$ $10^{-4} \mathrm{~g} / \mathrm{L}$ of air above the background levels detected. 
Table F-1. Data and analysis for the dust measurement test 1.

\begin{tabular}{|c|c|r|}
\hline Description & Monitor & \multicolumn{1}{c|}{$\begin{array}{c}\text { Dust loading } \\
\text { (g/L-air) }\end{array}$} \\
\hline \multirow{3}{*}{ Test Background } & HV-9 & $3.17 \mathrm{E}-07$ \\
\cline { 2 - 3 } & $H V-6$ & $8.22 \mathrm{E}-07$ \\
\cline { 2 - 3 } & HV-8 & $3.09 \mathrm{E}-07$ \\
\hline \multirow{5}{*}{ Test Samples } & HV-7 & $1.16 \mathrm{E}-06$ \\
\cline { 2 - 3 } & HV-8 & $3.12 \mathrm{E}-06$ \\
\cline { 2 - 3 } & HV-10 & $2.95 \mathrm{E}-06$ \\
\cline { 2 - 3 } & HV-17 & $5.06 \mathrm{E}-06$ \\
\cline { 2 - 3 } & HV-3 & $4.19 \mathrm{E}-06$ \\
\cline { 2 - 3 } & HV-9 & $1.67 \mathrm{E}-05$ \\
\hline
\end{tabular}

\begin{tabular}{|c|c|}
\hline & Basis: test bkg \\
\hline Test Mean $=$ & 5.54E-06 \\
\hline Test Std Dev $=$ & $5.65 \mathrm{E}-06$ \\
\hline $\mathrm{nd}=$ & 6 \\
\hline$n u=(n d+n b-2)=$ & 7 \\
\hline Critical 1 -Sided $t(.95, n u)=$ & $\overline{1.89}$ \\
\hline Pooled Std Dev $=$ & 4.77E-06 \\
\hline Critical Value t Statistic $=$ & -45.40 \\
\hline Calculated $P$-value $=$ & $\mathrm{n} / \mathrm{a}$ \\
\hline Background Mean = & $4.83 \mathrm{E}-07$ \\
\hline Background Std Dev $=$ & $2.94 \mathrm{E}-07$ \\
\hline $\mathrm{nb}=$ & 3 \\
\hline (Test Mean /Background Mean) $=$ & 11.47 \\
\hline
\end{tabular}

HV-\# = HI-VOL Air Sampler 


\section{Dust Measurement - Test \#2}

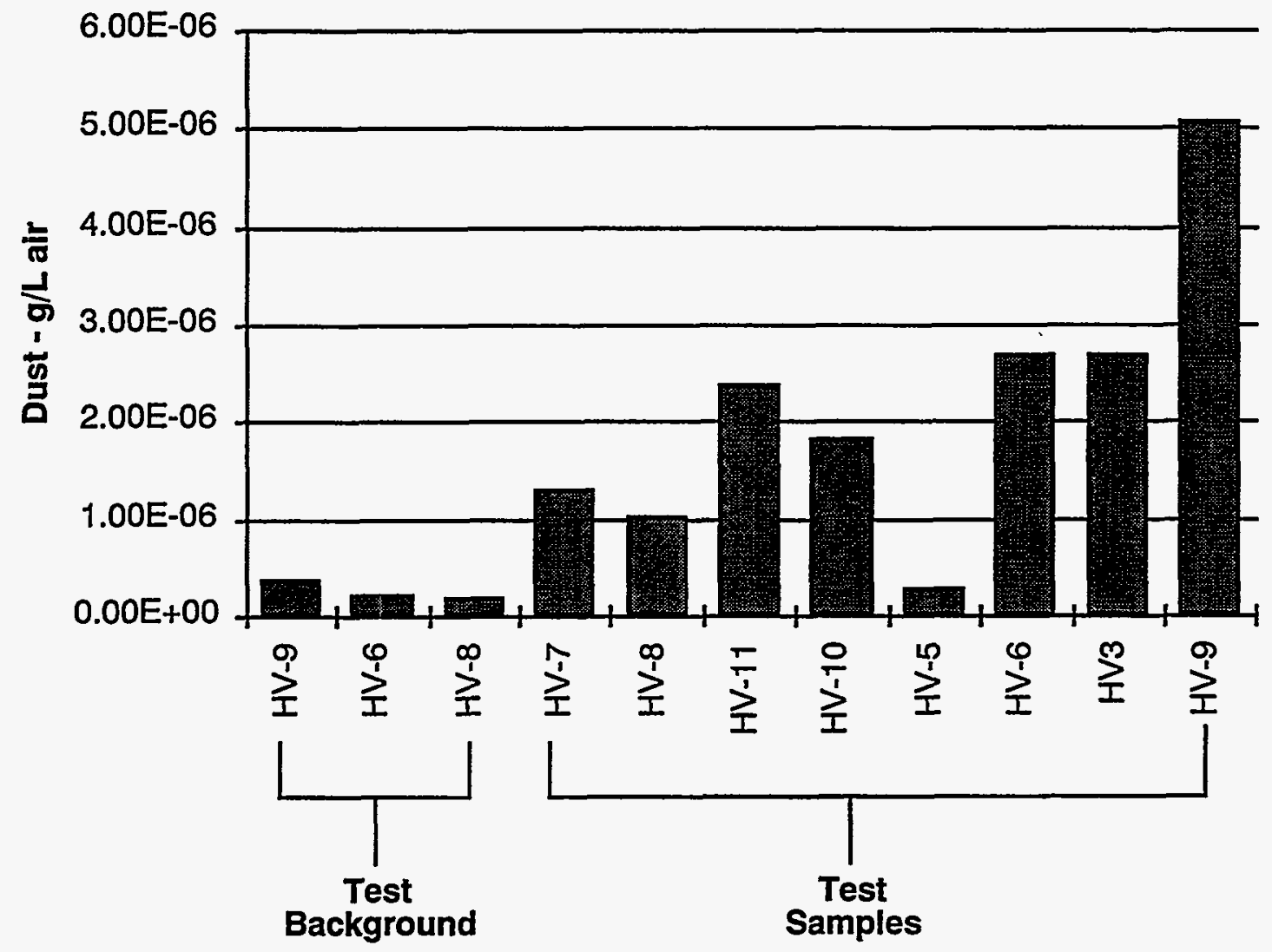

Air Sampler

Figure F-2. The dust measurement graph for test 2 shows the test sample levels were less than $1.1 \times$ $10^{-4} \mathrm{~g} / \mathrm{L}$ of air above the background levels detected. 
Table F-2. Data and analysis for the dust measurement test 2.

\begin{tabular}{|c|c|r|}
\hline \multicolumn{1}{|c|}{ Description } & Monitor & $\begin{array}{c}\text { Dust loading } \\
\text { (g/L-air) }\end{array}$ \\
\hline \multirow{3}{*}{ Test Background } & HV-9 & $3.75 \mathrm{E}-07$ \\
& HV-6 & $2.13 \mathrm{E}-07$ \\
\cline { 2 - 3 } & HV-8 & $1.77 \mathrm{E}-07$ \\
\cline { 2 - 3 } & HV-7 & $1.31 \mathrm{E}-06$ \\
\cline { 2 - 3 } & HV-8 & $1.02 \mathrm{E}-06$ \\
\cline { 2 - 3 } & HV-11 & $2.38 \mathrm{E}-06$ \\
\cline { 2 - 3 } & HV-10 & $1.83 \mathrm{E}-06$ \\
\cline { 2 - 3 } & HV-5 & $2.80 \mathrm{E}-07$ \\
\cline { 2 - 3 } & HV-6 & $2.68 \mathrm{E}-06$ \\
\cline { 2 - 3 } & HV3 & $2.70 \mathrm{E}-06$ \\
\cline { 2 - 3 } & HV-9 & $5.07 \mathrm{E}-06$ \\
\hline
\end{tabular}

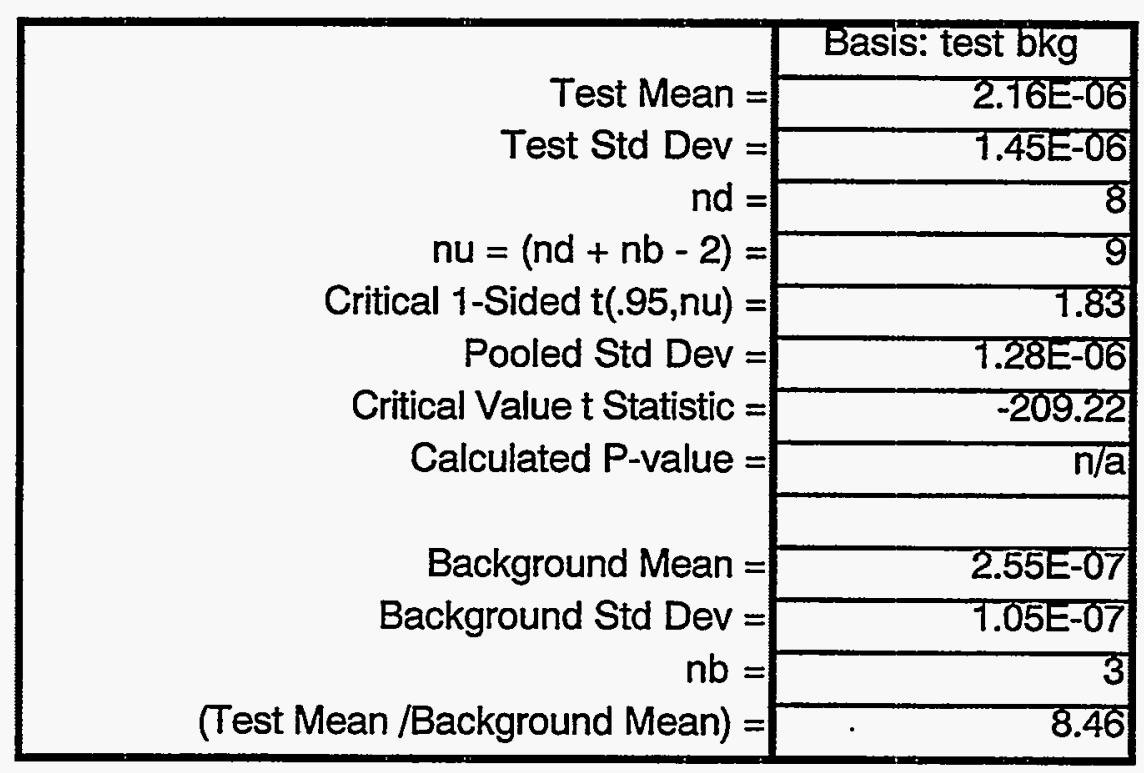

HV-\# = HI-VOL Air Sampler 
Dust Measurement - Test \#3

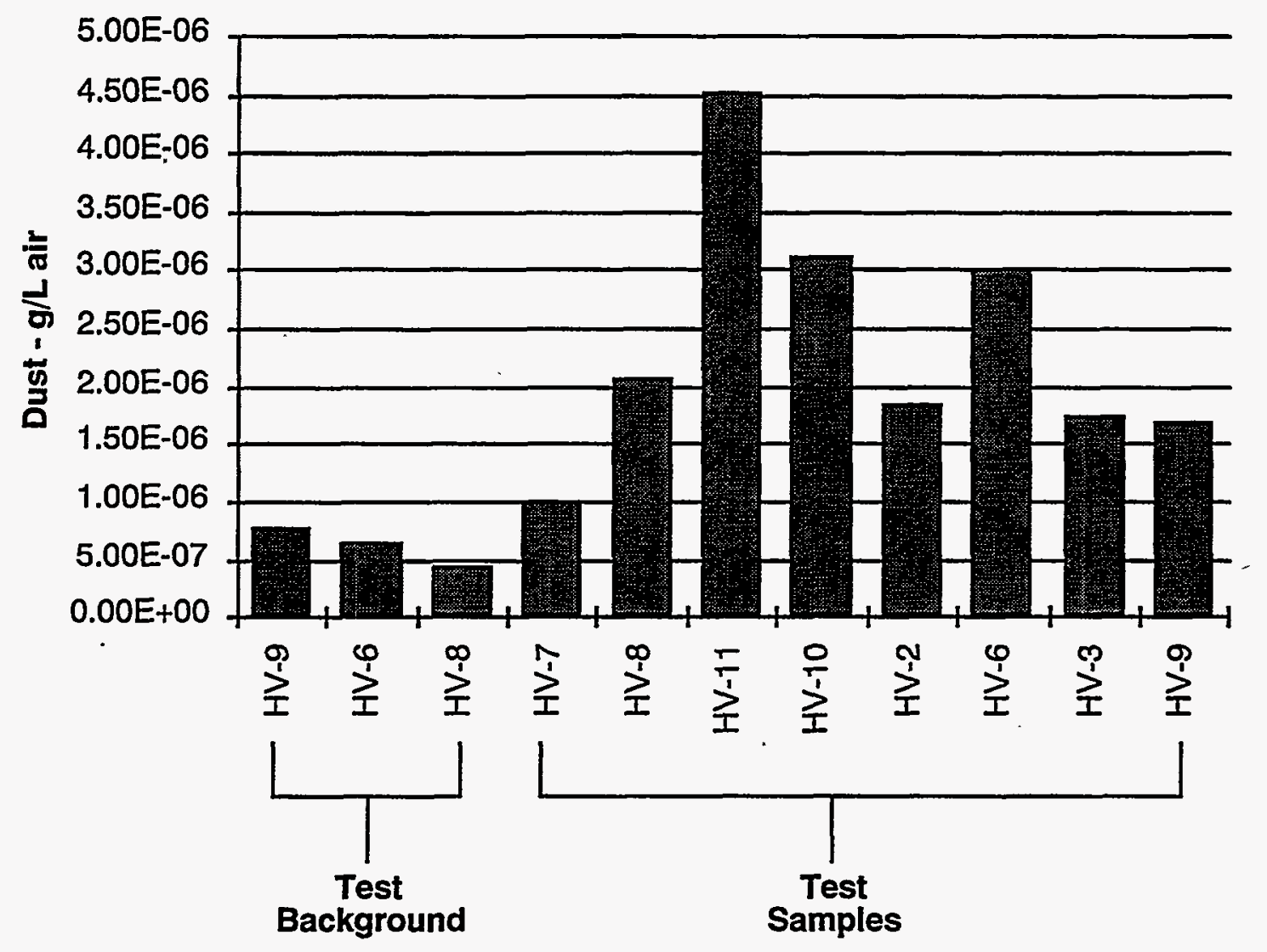

Air Sampler

Figure F-3. The dust measurement graph for test 3 shows the test sample levels were less then $1.1 \times$ $10^{-4} \mathrm{~g} / \mathrm{L}$ of air above the background levels detected. 
Table F-3. Data and analysis for the dust measurement test 3.

\begin{tabular}{|c|c|r|}
\hline Description & Monitor & $\begin{array}{c}\text { Dust loading } \\
\text { (g/L-air) }\end{array}$ \\
\hline \multirow{3}{*}{ Test Background } & HV-9 & $7.60 \mathrm{E}-07$ \\
\cline { 2 - 3 } & $\mathrm{HV}-6$ & $6.53 \mathrm{E}-07$ \\
\cline { 2 - 3 } & $\mathrm{HV}-8$ & $4.36 \mathrm{E}-07$ \\
\hline \multirow{5}{*}{ Test Samples } & $\mathrm{HV}-7$ & $9.97 \mathrm{E}-07$ \\
\cline { 2 - 3 } & $\mathrm{HV}-8$ & $2.08 \mathrm{E}-06$ \\
\cline { 2 - 3 } & $\mathrm{HV}-11$ & $4.51 \mathrm{E}-06$ \\
\cline { 2 - 3 } & $\mathrm{HV}-10$ & $3.11 \mathrm{E}-06$ \\
\cline { 2 - 3 } & $\mathrm{HV}-2$ & $1.85 \mathrm{E}-06$ \\
\cline { 2 - 3 } & $\mathrm{HV}-6$ & $3.01 \mathrm{E}-06$ \\
\cline { 2 - 3 } & $\mathrm{HV}-3$ & $1.73 \mathrm{E}-06$ \\
\cline { 2 - 3 } & $\mathrm{HV}-9$ & $1.69 \mathrm{E}-06$ \\
\hline
\end{tabular}

\begin{tabular}{|c|c|}
\hline & Basis: test bkg \\
\hline Test Mean $=$ & 2.37E-06 \\
\hline Test Std Dev $=$ & $1.11 \mathrm{E}-06$ \\
\hline $\mathrm{nd}=$ & 8 \\
\hline$n u=(n d+n b-2)=$ & $\overline{9}$ \\
\hline Critical 1-Sided $\mathrm{t}(.95, \mathrm{nu})=$ & 1.83 \\
\hline Pooled Std Dev $=$ & 9.83E-07 \\
\hline Critical Value $\mathrm{t}$ Statistic $=$ & -267.68 \\
\hline Calculated $\mathrm{P}$-value $=$ & $n / a$ \\
\hline Background Mean = & $6.16 \mathrm{E}-07$ \\
\hline Background Std Dev = & $1.65 \mathrm{E}-07$ \\
\hline $\mathrm{nb}=$ & 3 \\
\hline (Test Mean /Background Mean) $=$ & 3.85 \\
\hline
\end{tabular}

HV-\# = HI-VOL Air Sampler 


\section{Dust Measurement - Test \#4}

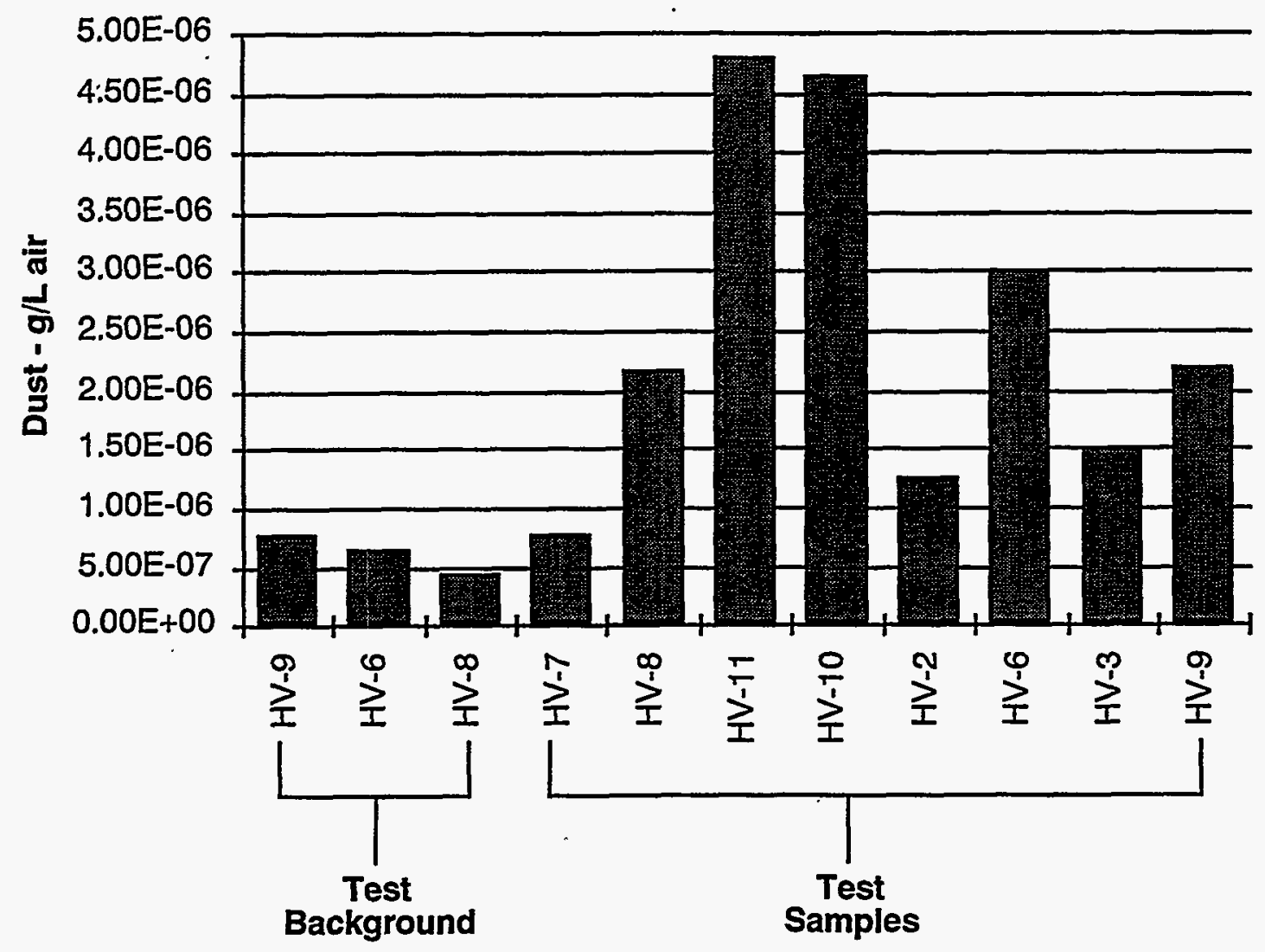

Air Sampler

Figure F-4. The dust measurement graph for test 4 shows the test sample levels were less then $1.1 \times$ $10^{-4} \mathrm{~g} / \mathrm{L}$ of air above the background levels detected. (Background samples from test 3 were included because test 4 was performed immediately following test 3.) 
Table F-4. Data and analysis for the dust measurement test 4.

\begin{tabular}{|c|c|c|}
\hline Description & Monitor & $\begin{array}{l}\text { Dust loading } \\
\text { (g/L-air) }\end{array}$ \\
\hline \multirow{3}{*}{ Test Background } & HV-9 & $7.60 \mathrm{E}-07$ \\
\hline & HV-6 & $6.53 \mathrm{E}-07$ \\
\hline & HV-8 & 4.36E-07 \\
\hline \multirow{8}{*}{ Test Samples } & HV-7 & $7.59 \mathrm{E}-07$ \\
\hline & HV-8 & $2.16 \mathrm{E}-06$ \\
\hline & HV-11 & $4.81 E-06$ \\
\hline & HV-10 & 4.64E-06 \\
\hline & HV-2 & $1.25 \mathrm{E}-06$ \\
\hline & HV-6 & 3.01E-06 \\
\hline & $\mathrm{HV}-3$ & 1.52E-06 \\
\hline & HV-9 & $2.19 \mathrm{E}-\mathrm{C}$ \\
\hline
\end{tabular}

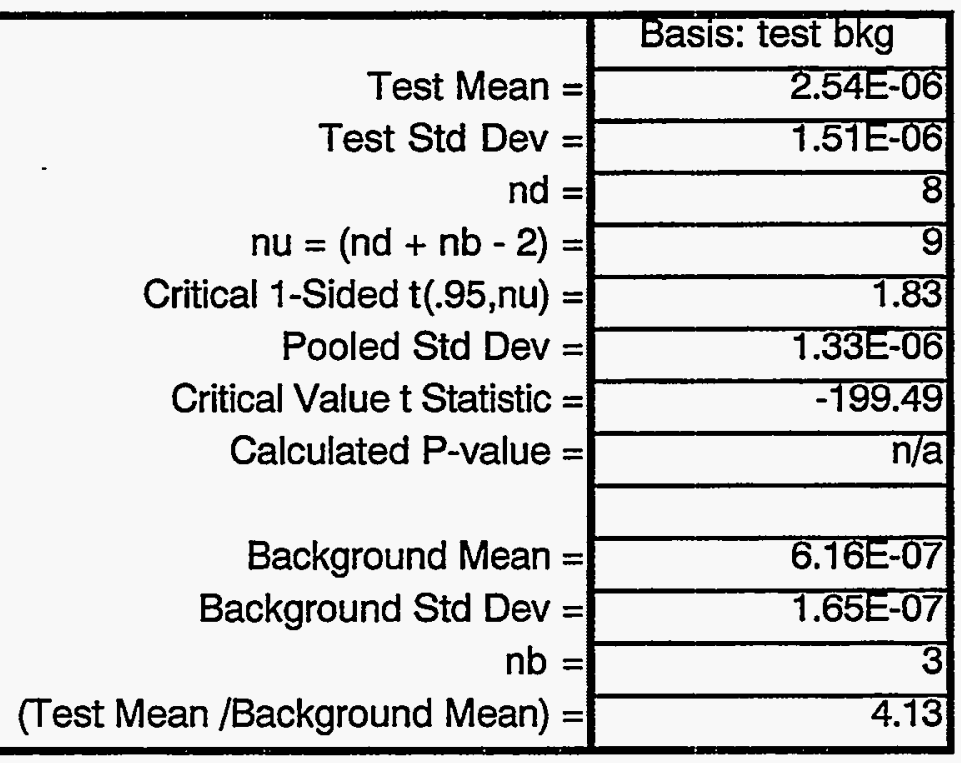

HV-\# = HI-VOL Air Sampler 


\section{Dust Measurement - Test \#5}

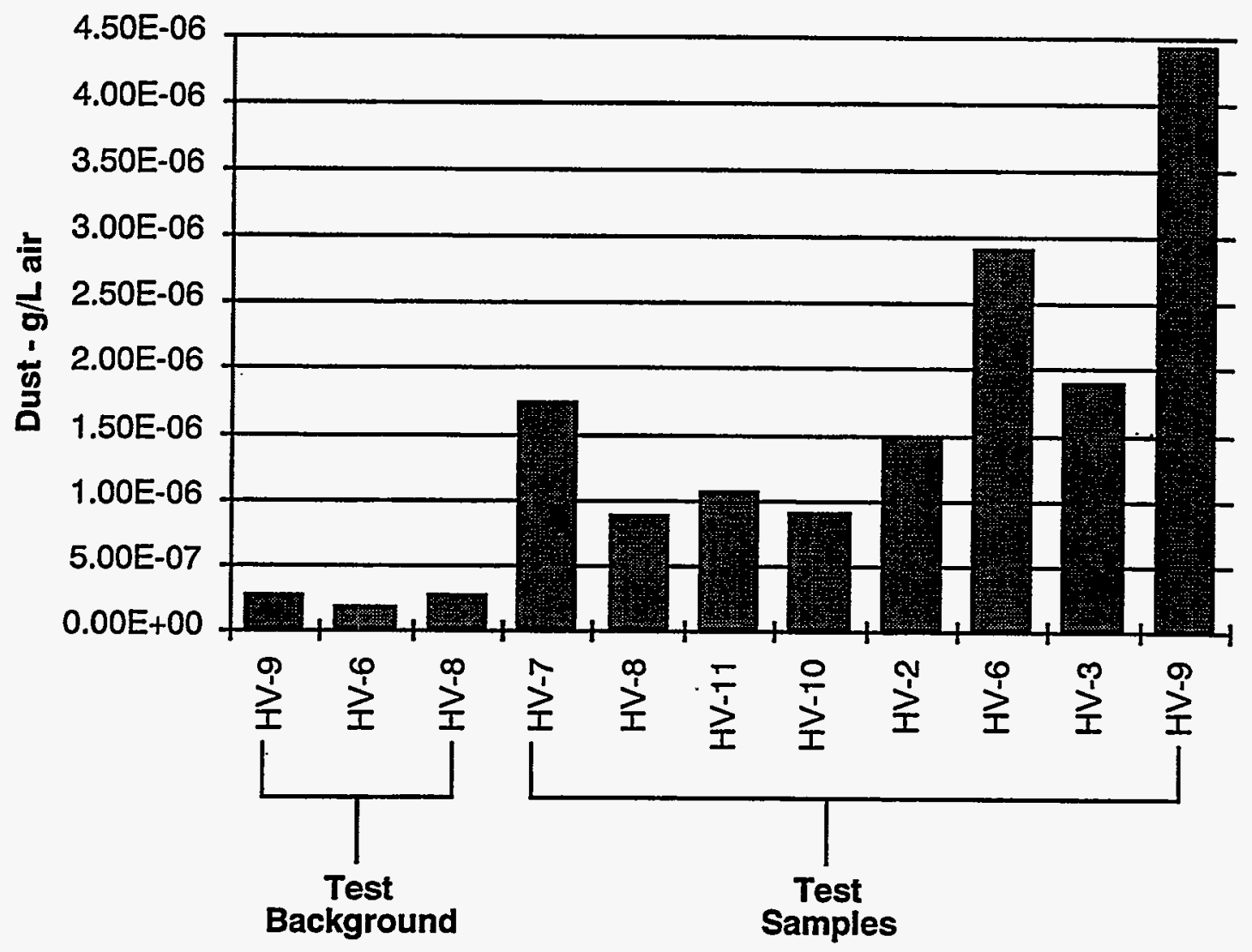

Air Sampler

Figure F-5. The dust measurement graph for test 5 shows the test sample levels were less then $1.1 \times$ $10^{-4} \mathrm{~g} / \mathrm{L}$ of air above the background levels detected. 
Table F-5. Data and analysis for the dust measurement test 5.

\begin{tabular}{|c|c|c|}
\hline Description & Monitor & $\begin{array}{l}\text { Dust loading } \\
\text { (g/L-air) }\end{array}$ \\
\hline \multirow{3}{*}{ Test Background } & HV-9 & 2.63E-07 \\
\hline & $H V-6$ & 1.87E-07 \\
\hline & HV-8 & 2.83E-07 \\
\hline \multirow{8}{*}{ Test Samples } & HV-7 & 1.75E-06 \\
\hline & HV-8 & 8.85E-07 \\
\hline & $\mathrm{HV}-11$ & 1.06E-06 \\
\hline & $H V-10$ & 9.15E-07 \\
\hline & HV-2 & 1.49E-06 \\
\hline & HV-6 & 2.92E-06 \\
\hline & HV-3 & $1.89 \mathrm{E}-06$ \\
\hline & HV-9 & 4.42E-06 \\
\hline
\end{tabular}

\begin{tabular}{|c|c|}
\hline & Basis: test bkg \\
\hline Test Mean $=$ & 1.92E-06 \\
\hline Test Std Dev $=$ & 7.21E-06 \\
\hline $\mathrm{nd}=$ & 8 \\
\hline$n u=(n d+n b-2)=$ & $\overline{9}$ \\
\hline Critical 1-Sided $t(.95, \mathrm{nu})=$ & -1.83 \\
\hline Pooled Std Dev = & 1.07E-06 \\
\hline Critical Value t Statistic $=$ & -252.18 \\
\hline Calculated P-value $=$ & $n / a$ \\
\hline Background Mear $=$ & $2.44 \mathrm{E}-07$ \\
\hline Background Std Dev = & 5.06E-08 \\
\hline $\mathrm{nb}=$ & 3 \\
\hline (Test Mean /Background Mean) $=$ & 7.84 \\
\hline
\end{tabular}

HV-\# = HI-VOL Air Sampler 
Conclusions: Since the t-statistic calculated in all cases is less than the critical 1-sided t test (see Table F-6), then the null hypothesis (Mean Dust Detected $\leq$ Background Dust $+1.1 \times 10^{-4} \mathrm{~g} / \mathrm{L}$ ) is not rejected. Thus the average amount of dust detected on the air sample filters was not greater than 1.1 $\times 10^{-4} \mathrm{~g} / \mathrm{L}$ of air above background levels for any of the tests in the demonstration. This conclusion is intuitively obvious since the observed dust loadings were two orders of magnitude less than the target goal of $1.1 \times 10^{-4} \mathrm{~g} / \mathrm{L}$.

Table F-6. The statistical analysis strongly supports the null hypothesis that the mean dust detected was, for all tests, less than the test goal of $98 \%$ dust removal.

\begin{tabular}{cccccc}
$\begin{array}{c}\text { Test } \\
\text { number }\end{array}$ & Calculated t statistic & $\begin{array}{c}\text { Critical 1-sided } \\
\mathrm{t}(.95, \mathrm{nu})\end{array}$ & Null hypothesis & $\begin{array}{c}\text { Do not reject } \\
\text { or reject }\end{array}$ \\
\hline 1 & -45.408 & 1.89 & $H_{o}:$ & $u_{d} \leq u_{b}+1.1 \times 10^{-4}$ & Do not reject \\
2 & -209.22 & 1.83 & $H_{o}:$ & $u_{d} \leq u_{b}+1.1 \times 10^{-4}$ & Do not reject \\
3 & -267.68 & 1.83 & $H_{o}:$ & $u_{d} \leq u_{b}+1.1 \times 10^{-4}$ & Do not reject \\
4 & -199.49 & 1.83 & $H_{o}:$ & $u_{d} \leq u_{b}+1.1 \times 10^{-4}$ & Do not reject \\
5 & -252.18 & 1.83 & $H_{O}:$ & $u_{d} \leq u_{b}+1.1 \times 10^{-4}$ & Do not reject \\
\hline
\end{tabular}

Discussion: The dust measurement for test 1 experienced the highest average amount of dust detected at $5.54 \mathrm{E}-06 \mathrm{~g} / \mathrm{L}$ compared with the lowest, Test 5 at $1.92 \mathrm{E}-06 \mathrm{~g} / \mathrm{L}$, or approximately $288 \%$ higher. See Figure F-6 for a comparison of the test averages and background averages.

\section{Dust Average by Test}

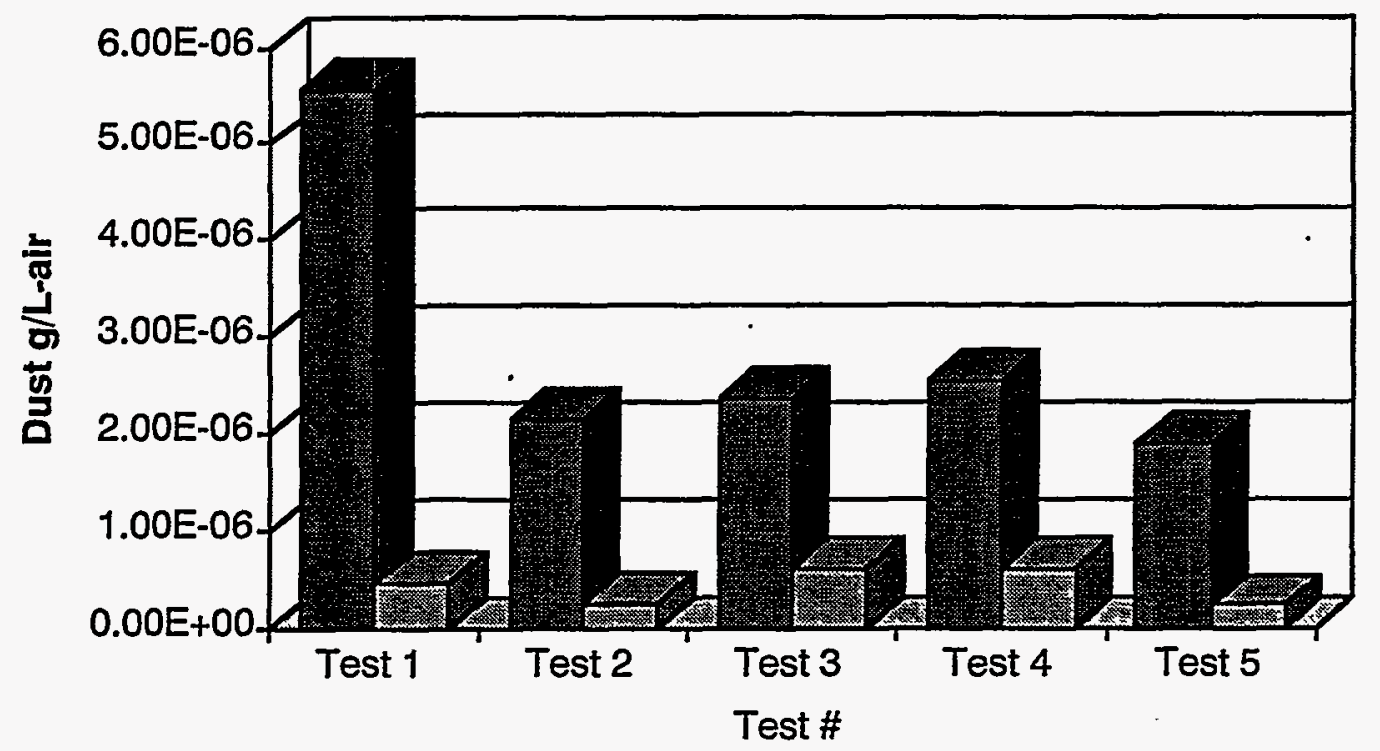

Test Mean

숭 Background Mean

Figure F-6. The test means for the 5 dust measurement tests clearly show that all of the dust samples taken are significantly (two orders of magnitude) below the target goal of $1.1 \times 10^{-4} \mathrm{~g} / \mathrm{L}$-air. 
The relatively high test average amounts for test 1 were primarily due to the high loadings from the HV-9. It was obvious from visual observations, and agreed upon by all those witnessing the demonstration that clearly virtually all of the dust generated and thus collected on the air monitors came from the digging operations. Virtually no dust was generated during the 'dumping' operations. This statement is supported by the data displayed in Figure F-7 which shows the average dust collected for each air monitor across all the tests. HV-9 (the air monitor closest to the digging in the path of the movement of the dust) shows significantly higher dust loadings than the rest of the air monitors. HV-9 was closest to the dig pile and along the direction of the prevailing movement of the dust cloud generated during digging (Figure F-8). This also supports why HV-7 has one of the lowest average dust loadings. Although it was also next to the pile, the dust cloud was always moving away from this air monitor and the waste wall partially shielded the monitor.

As previously discussed, much more dust was generated during the first test than any of the rest. The reason for this is that the operator of the excavator had very little practice with the innovative end effector prior to the start of the demonstration, and also had no experience digging in a waste pile like the one built for this demonstration. After the operator gained some experience, and the test conductors instructed him on how to more effectively retrieve the waste, the amount of dust generated was significantly reduced for the remainder of the demonstration.

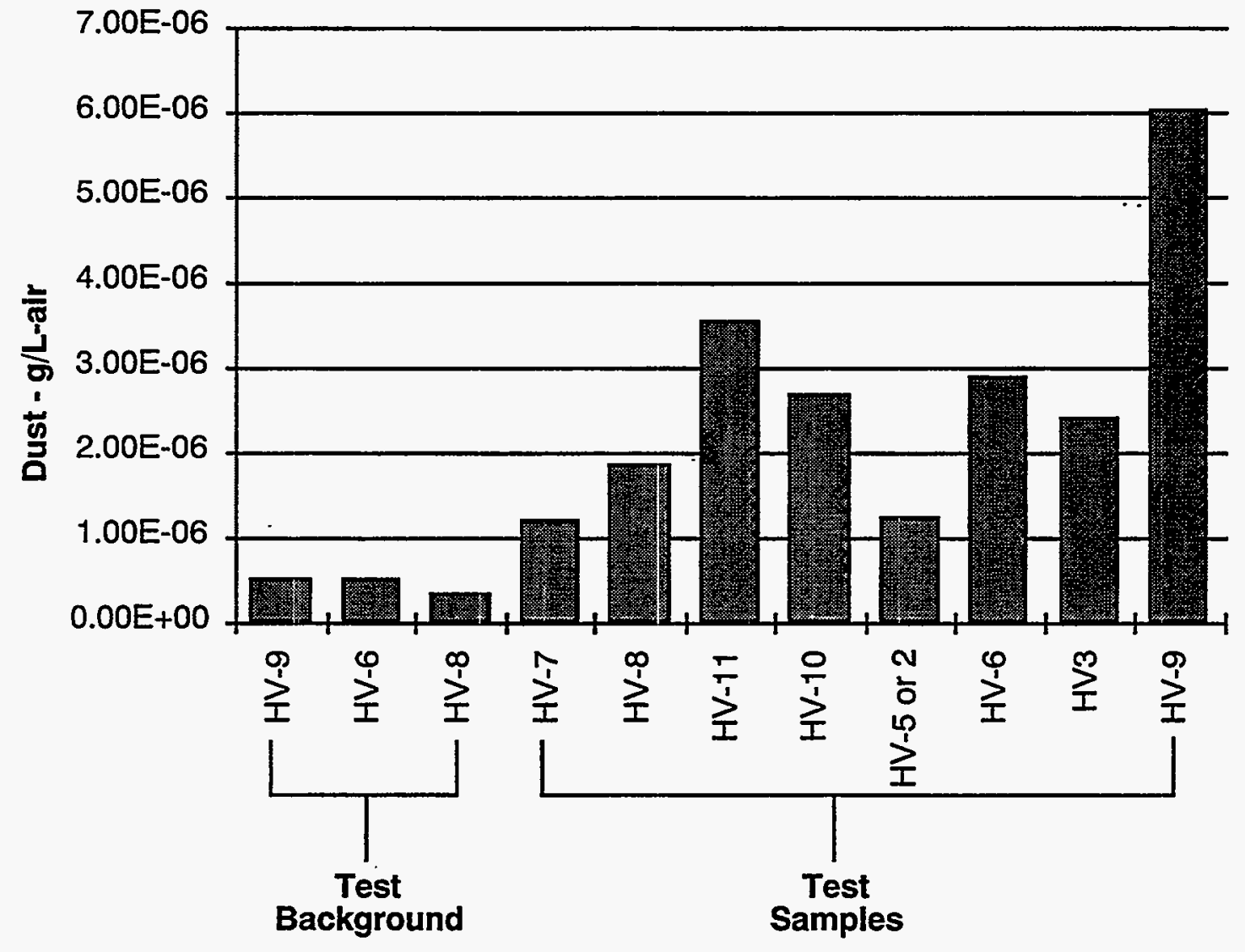

Monitor Number

Figure F-7. The average dust collected for each air monitor during the demonstration clearly shows that HV-9, the monitor closest to the digging, had the highest average dust loading. This clearly supports the visual observations that virtually all of the dust generation was a result of the digging operations, and that the dumping operations (or placement of the ITM) created virtually no dust. 


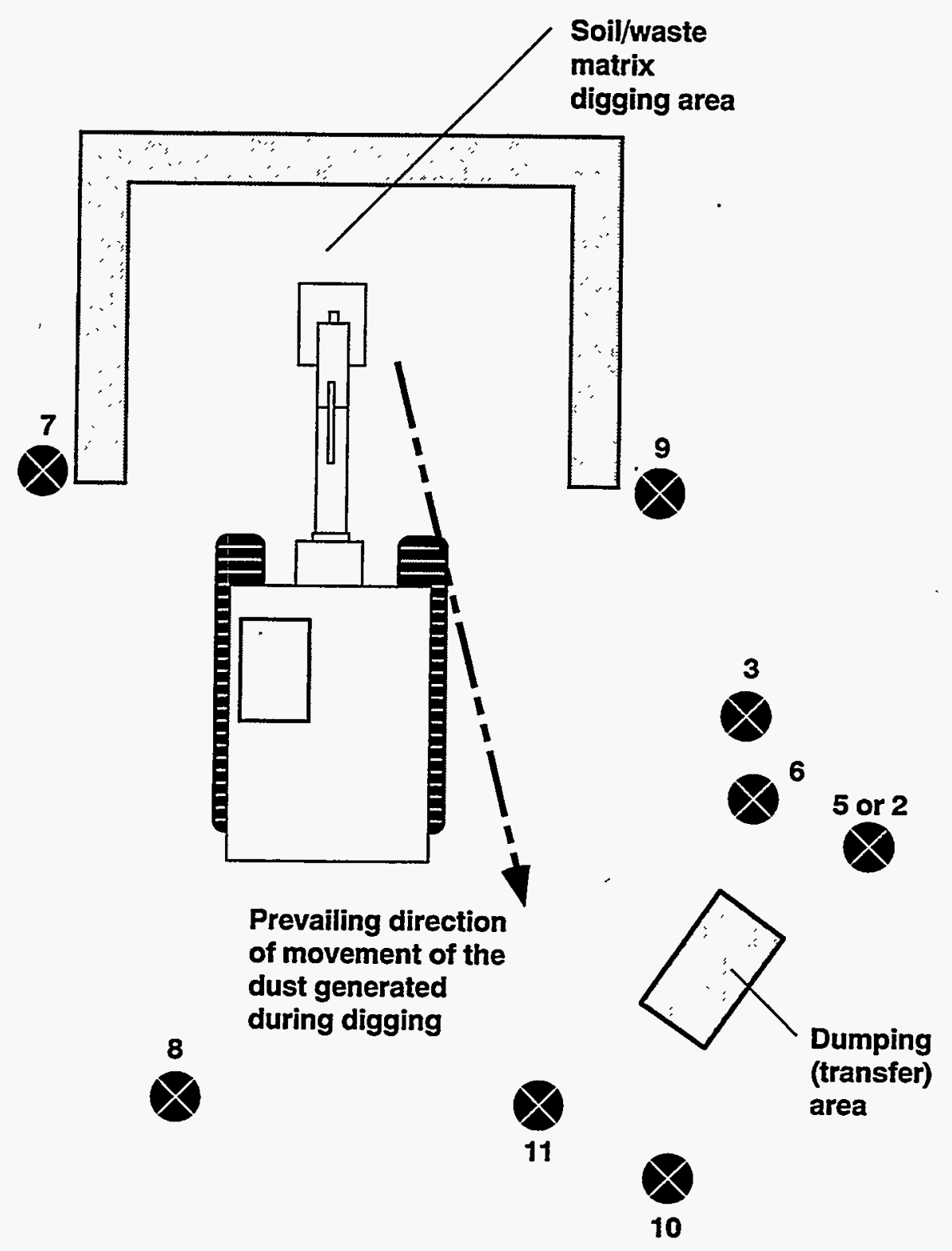

X94 0502

Figure F-8. Placement of the air monitors for the Phase I demonstration was intended to isolate the dust generation due to digging from that of the dumping. Prevailing air currents in the building carried the dust from the digging zone out across the test arena toward the dumping zone. However, the air monitor data (especially from HV-9) along with visual data clearly shows that the dumping operation was performed virtually dust free. 


\section{F-2. CONTAMINATION SPREAD}

\section{Analytical Procedure}

To assess whether the rare earth tracer (praseodymium) detected on the air sample filters exceeds the background level of praseodymium in the air, a statistical t-test was employed. The procedure tests the hypothesis of equality of the mean praseodymium (e.g., is the amount of praseodymium detected on the air sample filters equal the background level of praseodymium in the air in units of microgram-Pr/L-air?).

Null Hypothesis: $\quad H_{O}: \quad$ Mean Praseodymium Detected $=$ Background Praseodymium

or

Null Hypothesis: $\quad H_{O}: \quad u_{d}=u_{b}$

Alternate Hypothesis: $\quad H_{1}: \quad$ Mean Praseodymium Detected $>$ Background Praseodymium

or

Alternate Hypothesis: $\quad H_{1}: \quad u_{d}>u_{b}$

where

$u_{d} \quad=\quad$ the mean of the praseodymium measurements detected on the filters

$u_{b}=$ the mean of the background praseodymium measurements taken during the test

Because the two means are unknown, a pooled t-test or two-sample t-test will be used in the analysis. The assumption is that both distributions are normal.

The test statistic is therefore:

$$
t=\frac{\bar{x}_{d}-\bar{x}_{b}}{\sqrt{S_{d}^{2} / n_{d}+S_{b}^{2} / n_{b}}}
$$

where

$t=$ the critical value

$x_{d}=$ the observed mean of the praseodymium detected

$x_{b}=$ the observed mean of the background praseodymium detected

$n_{d} \quad=\quad$ the number of test filters

$n_{b} \quad=\quad$ the number of background filters

$S_{d}^{2} \quad=\quad$ the variance of the praseodymium detected

$S_{b}^{2} \quad=\quad$ the variance of the background praseodymium 
The null hypothesis $\left(H_{O}\right)$ is not rejected when:

$$
t<t\left(\alpha, n_{d}+n_{b}-2\right)
$$

where

$\alpha .=\quad$ level of significance

The null hypothesis $\left(H_{o}\right)$ is rejected in favor of the alternate hypothesis $\left(H_{1}\right)$ when:

$$
t>t\left(\alpha, n_{d}+n_{b}-2\right)
$$

Data Quality Objective: Determine with a 95\% confidence level if rare-earth tracer (praseodymium) was detected above background levels.

Test Data Analysis: Figures F-9 through F-13 show graphs of the praseodymium measured for the test backgrounds and test samples. The raw data, calculation of the mean and standard deviation, and the t-test analysis for each test are presented in Tables F-7 through F-11. 


\section{Rare Earth Tracer Measurement - Test \#1}

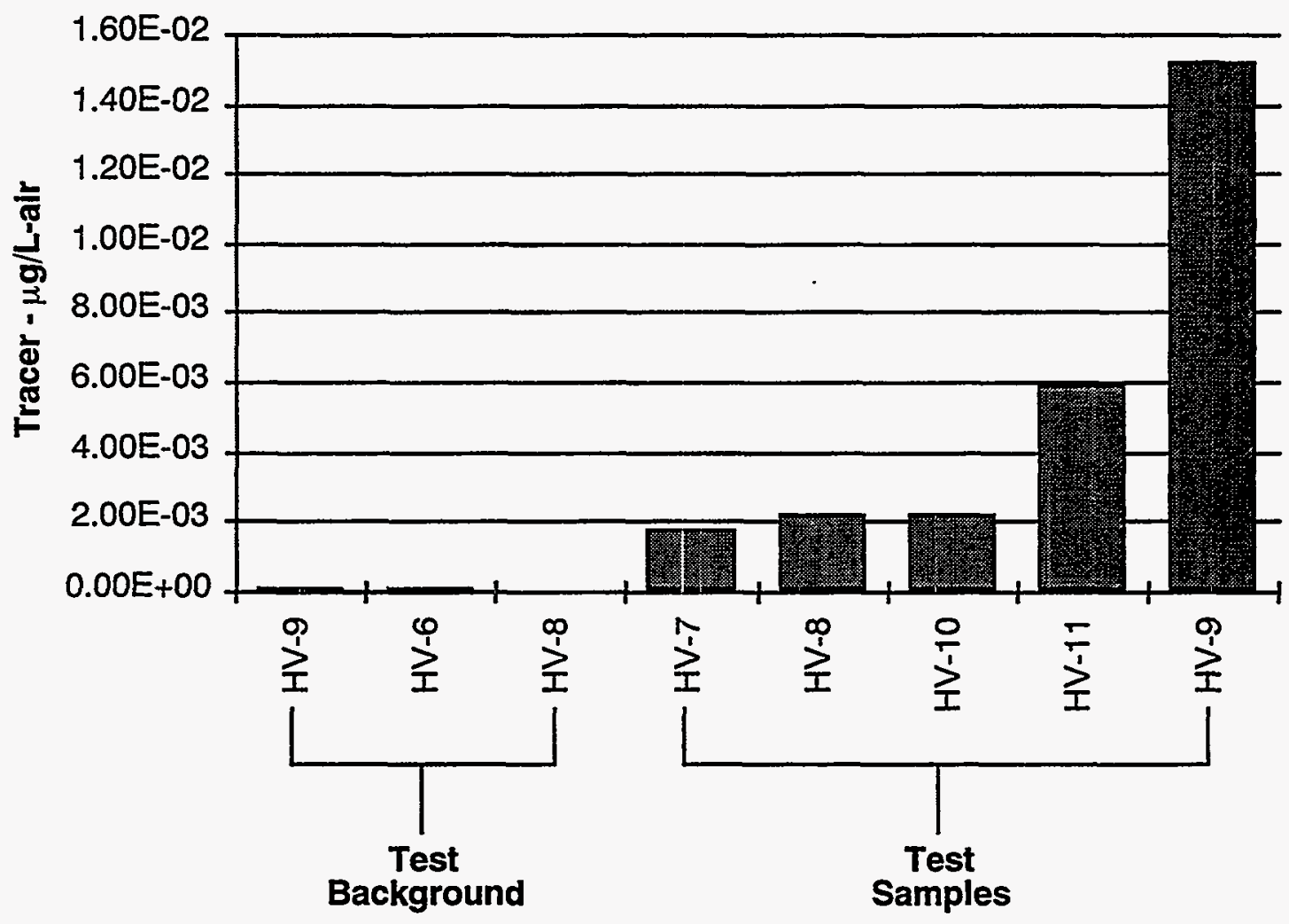

\section{Air Sampler}

Figure F-9. The rare earth tracer quantities graph for test 1 shows the praseodymium test sample levels and the background levels detected. 
Table F-7. Data and analysis for the rare earth tracer quantities test 1.

\begin{tabular}{|c|c|c|}
\hline Description & Monitor & $\begin{array}{c}\text { Tracerloading } \\
(\mu \mathrm{g} / \mathrm{L}-\mathrm{ir})\end{array}$ \\
\hline \multirow{3}{*}{ Test Background } & HV-9 & $1.78 \mathrm{E}-05$ \\
\cline { 2 - 3 } & $H V-6$ & $4.41 \mathrm{E}-05$ \\
\cline { 2 - 3 } & $H V-8$ & $1.09 \mathrm{E}-05$ \\
\hline \multirow{4}{*}{ Test Samples } & $H V-7$ & $1.73 \mathrm{E}-03$ \\
& $H V-8$ & $2.20 \mathrm{E}-03$ \\
\cline { 2 - 3 } & $H V-10$ & $2.20 \mathrm{E}-03$ \\
\cline { 2 - 3 } & $H V-11$ & $5.88 \mathrm{E}-03$ \\
\cline { 2 - 3 } & $H V-9$ & $1.52 \mathrm{E}-02$ \\
\hline
\end{tabular}

\begin{tabular}{|c|c|}
\hline & Basis: test bkg \\
\hline Test Mean $=$ & 5.44E-03 \\
\hline Test Std Dev $=$ & $5.70 \mathrm{E}-03$ \\
\hline & 5 \\
\hline degrees of freedom $=$ & 4 \\
\hline Critical 1-Sided $t(.95, n u)=$ & 2.13 \\
\hline Pooled Std Dev $=$ & $n / a$ \\
\hline Critical Value t Statistic $=$ & 2.12 \\
\hline Calculated P-value $=$ & $\mathrm{n} / \mathrm{a}$ \\
\hline Background Mean $=$ & $2.43 E-05$ \\
\hline Background Std Dev $=$ & 1.75E-05 \\
\hline $\mathrm{nb}=$ & 3 \\
\hline$($ Test Mean /Background Mean) $=$ & 224.19 \\
\hline
\end{tabular}

HV-\# = HI-VOL Air Sampler 


\section{Rare Earth Tracer Quantities - Test \#2}

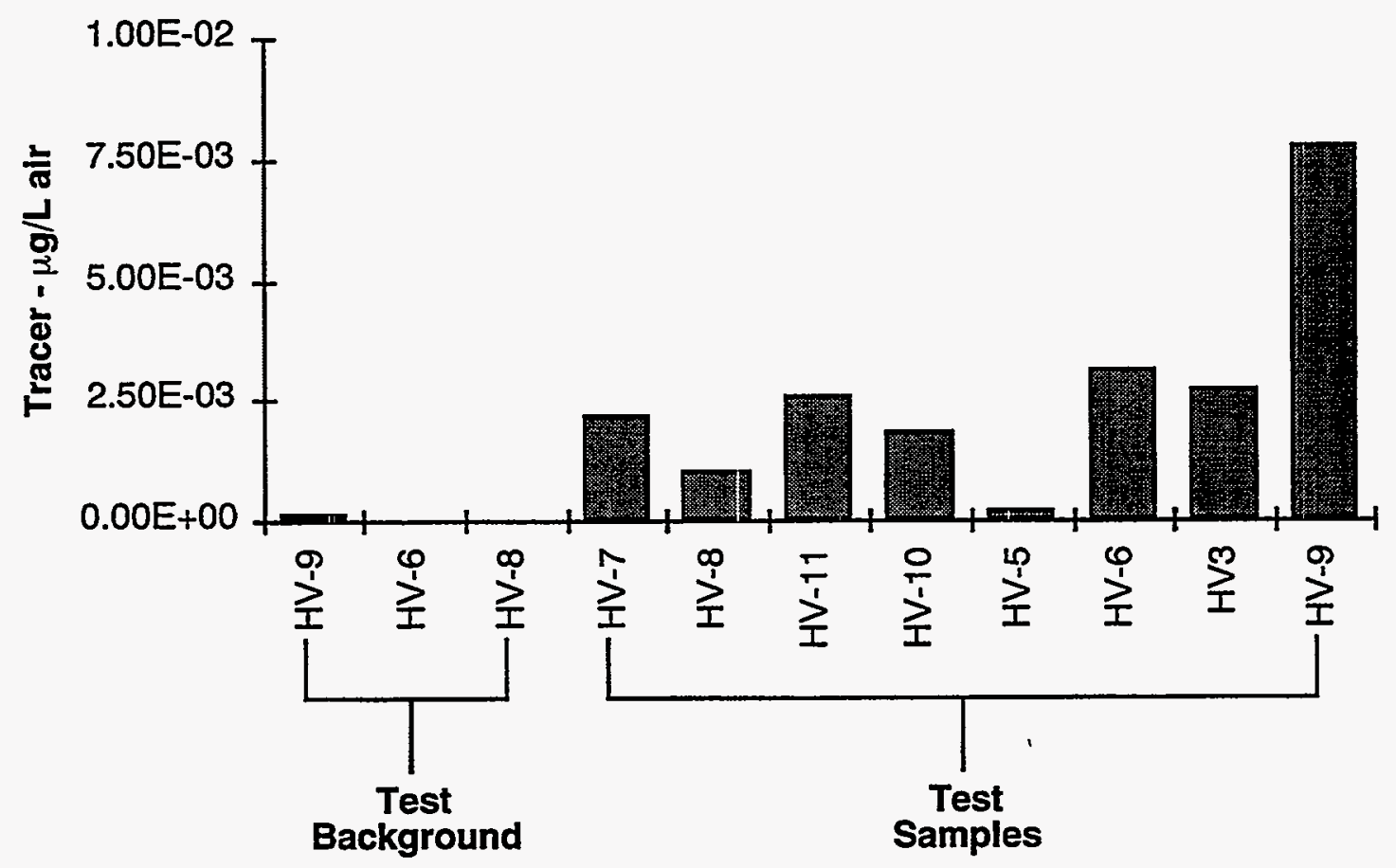

\section{Air Sampler}

Figure F-10. The rare earth tracer quantities graph for test 2 shows the praseodymium test sample levels and the background levels detected. 
Table F-8. Data and analysis for the rare earth tracer quantities test 2.

\begin{tabular}{|c|c|c|}
\hline Description & Monitor & $\begin{array}{l}\text { Tracer loading } \\
\text { ( } \mu \mathrm{g} / \mathrm{L} \text {-air) }\end{array}$ \\
\hline \multirow{3}{*}{ Test Background } & HV-9 & $1.28 \mathrm{E}-04$ \\
\hline & HV-6 & 1.12E-05 \\
\hline & HV-8 & 6.25E-06 \\
\hline \multirow{8}{*}{ Test Samples } & $\mathrm{HV}-7$ & $2.18 \mathrm{E}-03$ \\
\hline & HV-8 & $7.05 E-03$ \\
\hline & $H V-11$ & $2.61 E-03$ \\
\hline & HV-10 & 1.83E-03 \\
\hline & HV-5 & 2.44E-04 \\
\hline & HV-6 & $3.10 \mathrm{E}-03$ \\
\hline & $\mathrm{HV} 3$ & 2.72E-03 \\
\hline & HV-9 & 7.78E-03 \\
\hline
\end{tabular}

\begin{tabular}{|c|c|}
\hline \multirow{13}{*}{$\begin{aligned} \text { Test Mean } & = \\
\text { Test Std Dev } & = \\
\text { nd } & = \\
\text { degrees of freedom } & = \\
\text { Critical 1-Sided } t(.95, \mathrm{nu}) & = \\
\text { Pooled Std Dev } & = \\
\text { Critical Value t Statistic } & = \\
\text { Calculated P-value } & = \\
\text { Background Mean } & = \\
\text { Background Std Dev } & = \\
\mathrm{nb} & = \\
\text { (Test Mean /Background Mean) } & =\end{aligned}$} & Basis: test bkg \\
\hline & $2.69 \mathrm{E}-03$ \\
\hline & $2.26 \mathrm{E}-03$ \\
\hline & \\
\hline & \\
\hline & 7.89 \\
\hline & $n / a$ \\
\hline & 3.30 \\
\hline & $\pi / a$ \\
\hline & $4.86 E-05$ \\
\hline & $6.90 \mathrm{E}-05$ \\
\hline & \\
\hline & 55.39 \\
\hline
\end{tabular}

HV-\# = HI-VOL Air Sampler 


\section{Rare Earth Tracer Quantities - Test \#3}

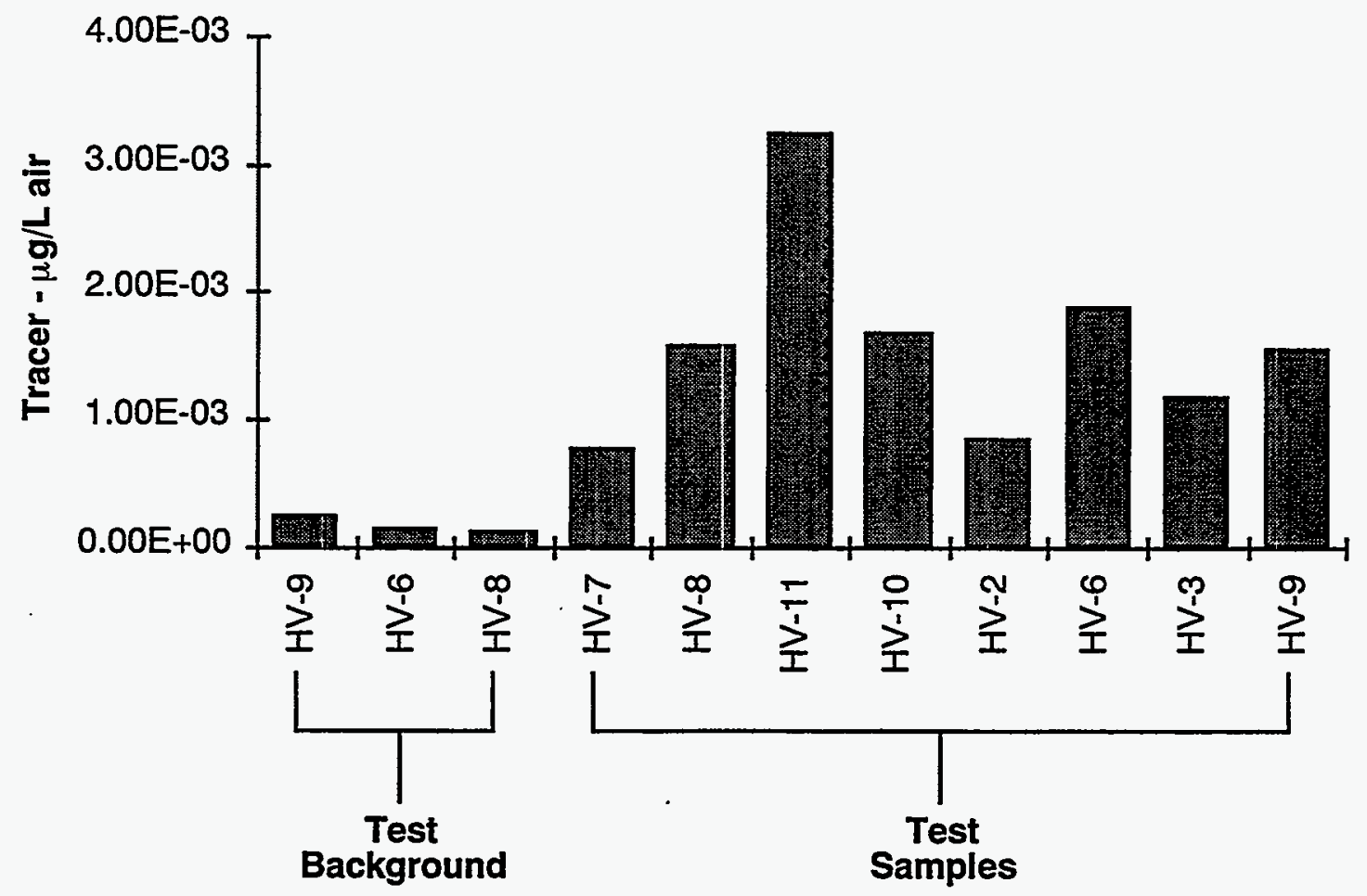

Air Sampler

Figure F-11. The rare earth tracer quantities graph for test 3 shows the praseodymium test sample levels and the background levels detected. 
Table F-9. Data and analysis for the rare earth tracer quantities test 3.

\begin{tabular}{|c|c|c|}
\hline Description & Monitor & $\begin{array}{c}\text { Tracer loading } \\
\langle\mu \mathrm{g} / \mathrm{L}-\mathrm{Lir})\end{array}$ \\
\hline \multirow{3}{*}{ Test Background } & $\mathrm{HV}-9$ & $2.45 \mathrm{E}-04$ \\
\cline { 2 - 3 } & $\mathrm{HV}-6$ & $1.56 \mathrm{E}-04$ \\
\cline { 2 - 3 } & $\mathrm{HV}-8$ & $1.20 \mathrm{E}-04$ \\
\hline \multirow{5}{*}{ Test Samples } & $\mathrm{HV}-7$ & $7.84 \mathrm{E}-04$ \\
\cline { 2 - 3 } & $\mathrm{HV}-8$ & $1.57 \mathrm{E}-03$ \\
\cline { 2 - 3 } & $\mathrm{HV}-11$ & $3.24 \mathrm{E}-03$ \\
\cline { 2 - 3 } & $\mathrm{HV}-10$ & $1.68 \mathrm{E}-03$ \\
\cline { 2 - 3 } & $\mathrm{HV}-2$ & $8.63 \mathrm{E}-04$ \\
\cline { 2 - 3 } & $\mathrm{HV}-6$ & $1.88 \mathrm{E}-03$ \\
\cline { 2 - 3 } & $\mathrm{HV}-3$ & $1.17 \mathrm{E}-03$ \\
\cline { 2 - 3 } & $\mathrm{HV}-9$ & $1.55 \mathrm{E}-03$ \\
\hline
\end{tabular}

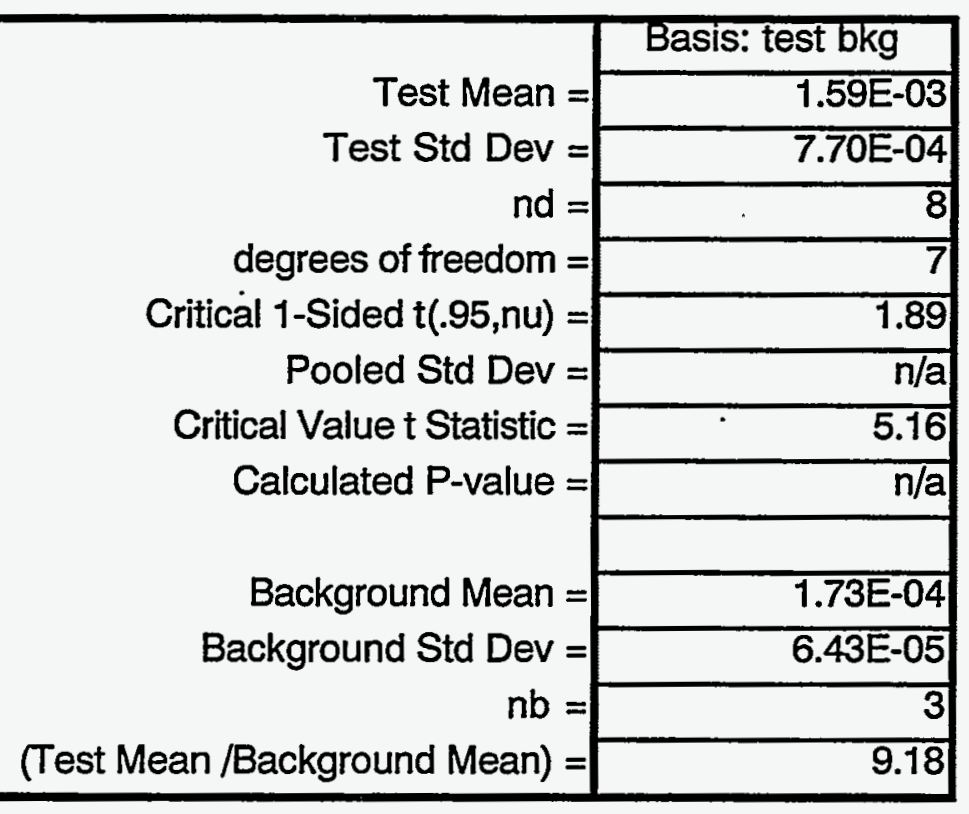

HV-\# = HI-VOL Air Sampler 


\section{Rare Earth Tracer Quantities - Test \#4}

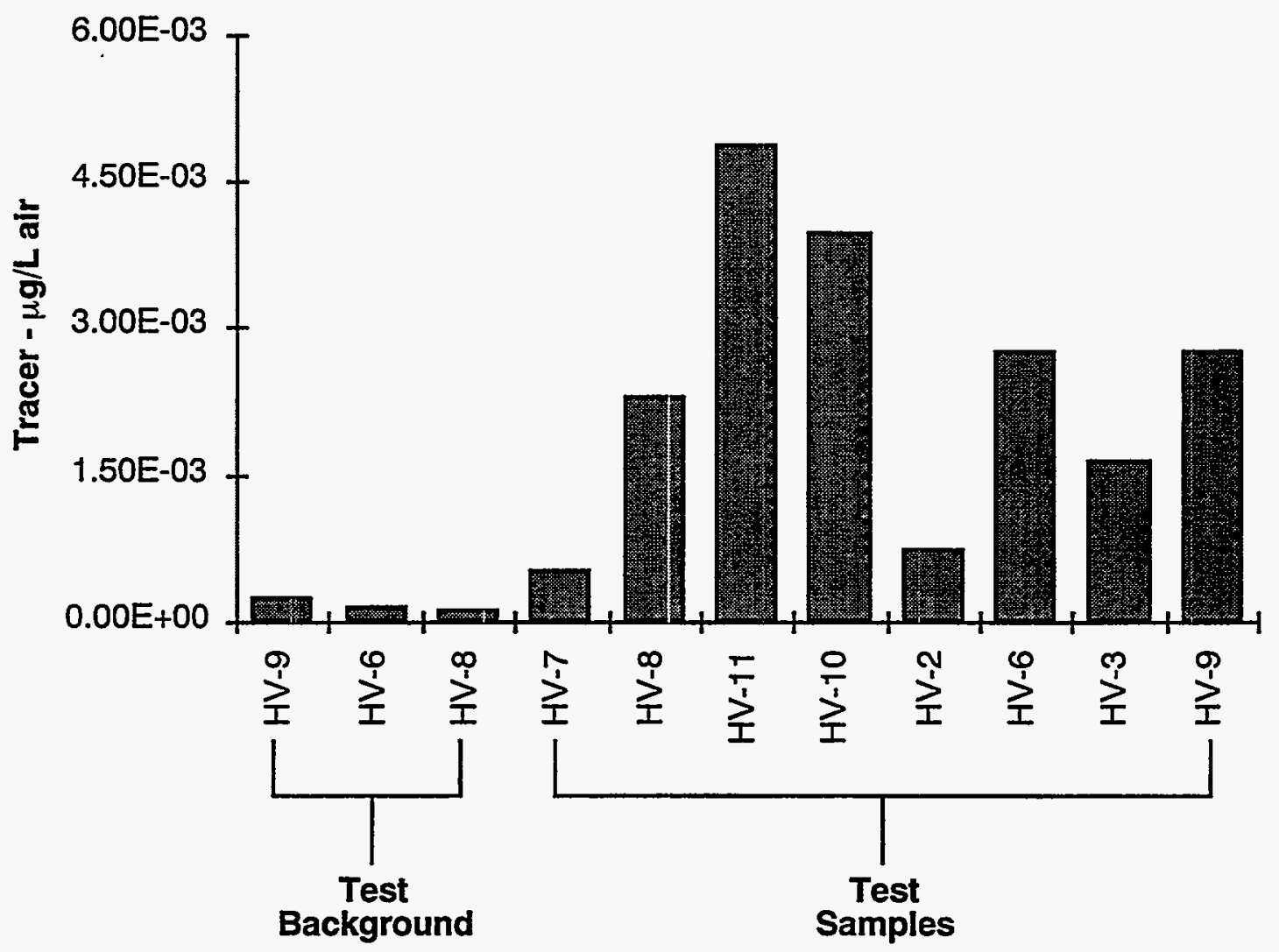

Air Sampler

Figure F-12. The rare earth tracer quantities graph for test 4 shows the praseodymium test sample levels and the background levels detected. (Background samples from Test 3 were included because Test 4 was performed shortly after Test 3.) 
Table F-10. Data and analysis for the rare earth tracer quantities test 4.

\begin{tabular}{|c|c|c|}
\hline Description & Monitor & $\begin{array}{l}\text { Tracer loading } \\
(\mu \mathrm{g} / \mathrm{L} \text {-air) }\end{array}$ \\
\hline \multirow{3}{*}{ Test Background } & HV-9 & 2.45E-04 \\
\hline & HV-6 & $1.56 \mathrm{E}-04$ \\
\hline & HV-8 & $1.20 E-04$ \\
\hline \multirow{8}{*}{ Test Samples } & $\mathrm{HV}-7$ & $5.23 \mathrm{E}-04$ \\
\hline & HV-8 & $2.30 \mathrm{E}-03$ \\
\hline & HV-11 & $4.88 \mathrm{E}-03$ \\
\hline & HV-10 & $3.99 \mathrm{E}-03$ \\
\hline & HV-2 & $7.46 E-04$ \\
\hline & HV-6 & 2.77E-03 \\
\hline & HV-3 & 1.65E-03 \\
\hline & HV-9 & $2.76 \mathrm{E}-03$ \\
\hline
\end{tabular}

\begin{tabular}{|c|c|}
\hline & Basis: test bkg \\
\hline Test Mean $=$ & $2.45 E-03$ \\
\hline Test Std Dev $=$ & $7.50 \mathrm{E}-03$ \\
\hline & \\
\hline degrees of freedom $=$ & \\
\hline Critical 1 -Sided $t(.95, \mathrm{nu})=$ & 7.89 \\
\hline Pooled Std Dev $=$ & $n / a$ \\
\hline Critical Value t Statistic $=$ & 4.29 \\
\hline Calculated $P$-value $=$ & $\mathrm{n} / \mathrm{a}$ \\
\hline Background Mean = & $1.73 \mathrm{E}-04$ \\
\hline Background Std Dev = & 6.43E-05 \\
\hline $\mathrm{nb}=$ & 3 \\
\hline (Test Mean /Background Mean) = & 14.13 \\
\hline
\end{tabular}

HV-\# = HI-VOL Air Sampler 
Rare Earth Tracer Quantities - Test \#5

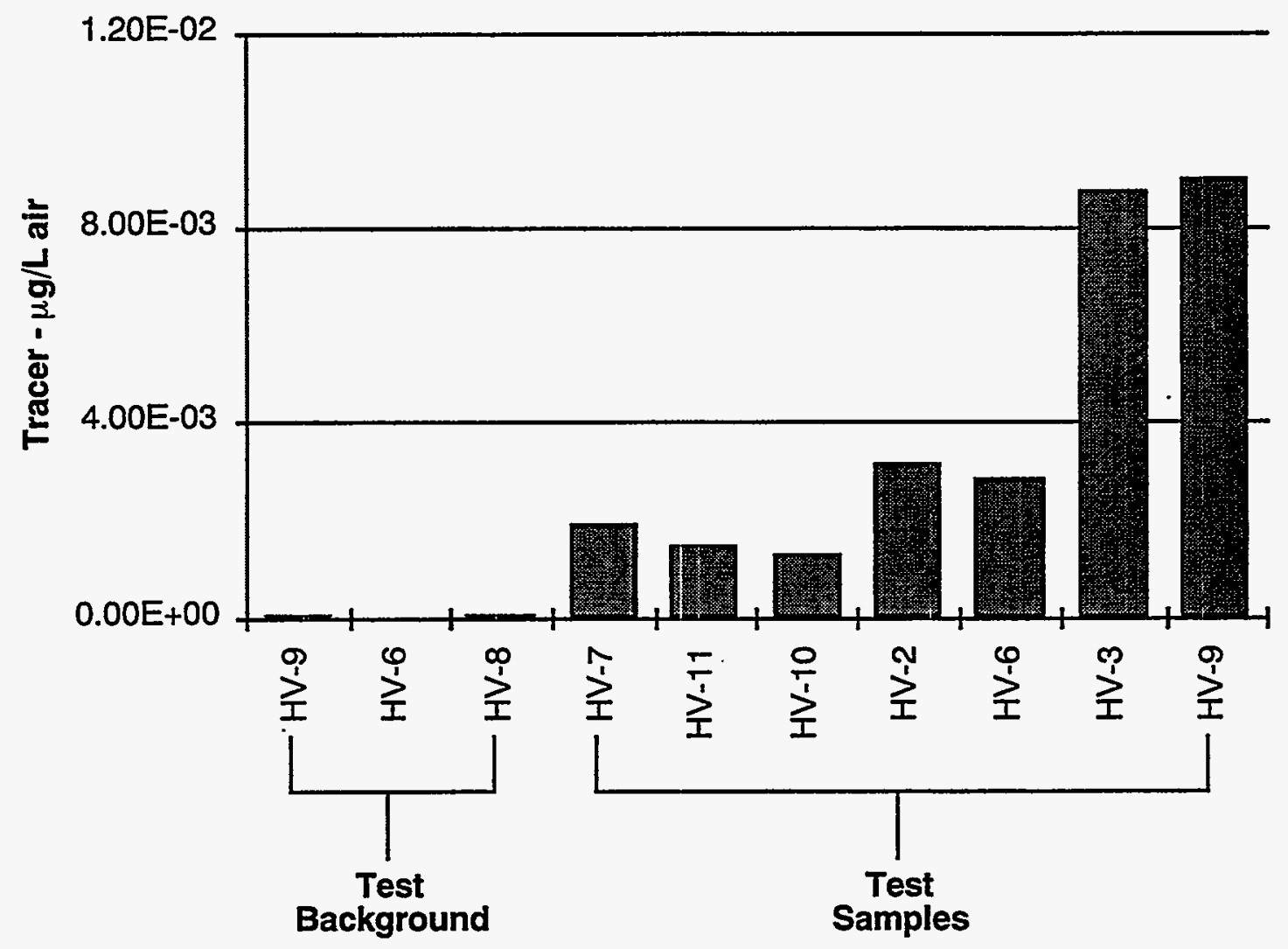

Air Sampler

Figure F-13. The rare earth tracer quantities graph for test 5 shows the praseodymium test sample levels and the background levels detected. 
Table F-11. Data and analysis for the rare earth tracer quantities test 5.

\begin{tabular}{|c|c|c|}
\hline Description & Monitor & $\begin{array}{l}\text { Tracer loading } \\
\text { ( } \mathrm{g} / \mathrm{L} \text {-air) }\end{array}$ \\
\hline \multirow{3}{*}{ Test Background } & HV-9 & $3.60 \mathrm{E}-05$ \\
\hline & HV-6 & 7.70E-05 \\
\hline & HV-8 & 3.01E-05 \\
\hline \multirow{7}{*}{ Test Samples } & HV-7 & $1.93 \mathrm{E}-03$ \\
\hline & HV-11 & $1.48 \mathrm{E}-03$ \\
\hline & HV-10 & $1.32 \mathrm{E}-03$ \\
\hline & $H V-2$ & $3.19 \mathrm{E}-03$ \\
\hline & HV-6 & $2.84 E-03$ \\
\hline & $H V-3$ & $8.78 \mathrm{E}-03$ \\
\hline & HV-9 & 9.01E-03 \\
\hline
\end{tabular}

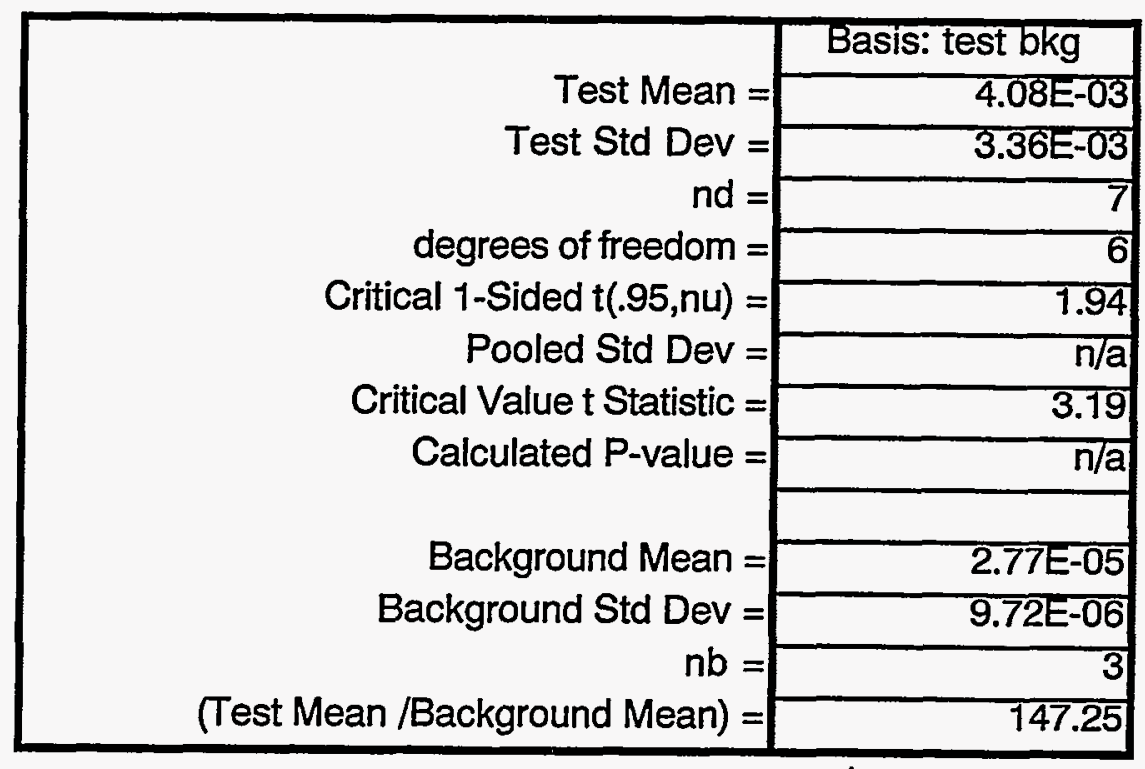

HV-\# = HI-VOL Air Sampler 
Conclusions: The t-statistic calculated in all but one case is less than the critical 1-sided t-test (see Table F-12). The null hypothesis (Mean Praseodymium Detected = Background Praseodymium) is therefore rejected in favor of the alternative hypothesis (Mean Praseodymium Detected > Background Praseodymium). Thus the average amount of Praseodymium detected on the air sample filters was greater than background levels for four of the five tests in the demonstration. Failure to determine that the tracer detected exceeded the background levels for the first test is a statistical problem caused by the failure to meet the data quality objective for this test. This was due to a power failure that caused several of the air monitors to prematurely shut down. Statistically speaking, the lack of enough data and the high variance of the data collected made it impossible to determine if the tracer detected was higher than background. Practically speaking, the data obviously shows that the tracer levels detected were above background. This is indicative of a failure to meet a data quality objective (i.e., the quality of the data was not enough to make a statistically significant determination of the results).

Table F-12. The statistical analysis supports the alternative hypothesis that the mean tracer detected was, for most tests, greater than the background levels.

\begin{tabular}{|c|c|c|c|c|}
\hline $\begin{array}{c}\text { Test } \\
\text { number }\end{array}$ & Calculated $\mathrm{t}$ statistic & $\begin{array}{c}\text { Critical 1-sided } \\
\mathrm{t}(.95, \mathrm{nu})\end{array}$ & Null hypothesis & $\begin{array}{c}\text { Do not reject } \\
\text { or reject }\end{array}$ \\
\hline 1 & 2.12 & 2.13 & $H_{O}: \quad u_{d}=u_{b}$ & Do not reject \\
\hline 2 & 3.30 & 1.83 & $H_{o}: \quad u_{d}=u_{b}$ & Reject \\
\hline 3 & 5.16 & 1.83 & $H_{O}: \quad u_{d}=u_{b}$ & Reject \\
\hline 4 & 4.29 & 1.83 & $H_{o}: \quad u_{d}=u_{b}$ & Reject \\
\hline 5 & 3.19 & 1.86 & $H_{o}: \quad u_{d}=u_{b}$ & Reject \\
\hline
\end{tabular}

Discussion: The rare earth tracer measurement for test 1 experienced the highest average amount of tracer detected at $5.44 \times 10^{-3} \mu \mathrm{g} / \mathrm{L}$-air compared with the lowest, test 3 at $1.59 \times 10^{-3} \mu \mathrm{g} / \mathrm{L}$-air. See Figure F-14 for a comparison of the test averages and background averages.

For all sampler positions, analysis of the filters for tracer concentration showed that tracer spread to all sampler positions during the combined digging/dumping operation. All filter positions showed tracer concentrations above background as shown in Figure F-15. For all five tests, the ratio of the test mean data to the background mean data is greater than 1 . The range of rare earth tracer concentrations on the filters was between 9.18 and 224.19 times the background levels. Again, as with the dust data, the average tracer collected for each air monitor during the demonstration (Figure F-15) clearly shows that HV-9, the monitor closest to the digging, had the highest average tracer loading. This clearly supports the visual observations that virtually all of the dust generation was a result of the digging operations, and that the dumping operations (or placement of the ITM) created virtually no dust. (Note: Only simple statistical techniques were used to examine the relationships between each dust monitor and across tests. Assumptions about the variance across tests were made based on similar patterns of dust and tracer loading for each test and upon visual observations. More sophisticated statistical techniques could yield interesting data relationships, however the capability of the author to perform these tests (minimal), and the availability and cost of statisticians to perform more thorough analysis, prohibited expansion of the analysis. A statistician was consulted and 
reviewed the results. There did not seam to be a great deal of value in performing a more in-depth statistical study of the results. This will be left to the reader).

\section{Rare Earth Tracer Average by Test}

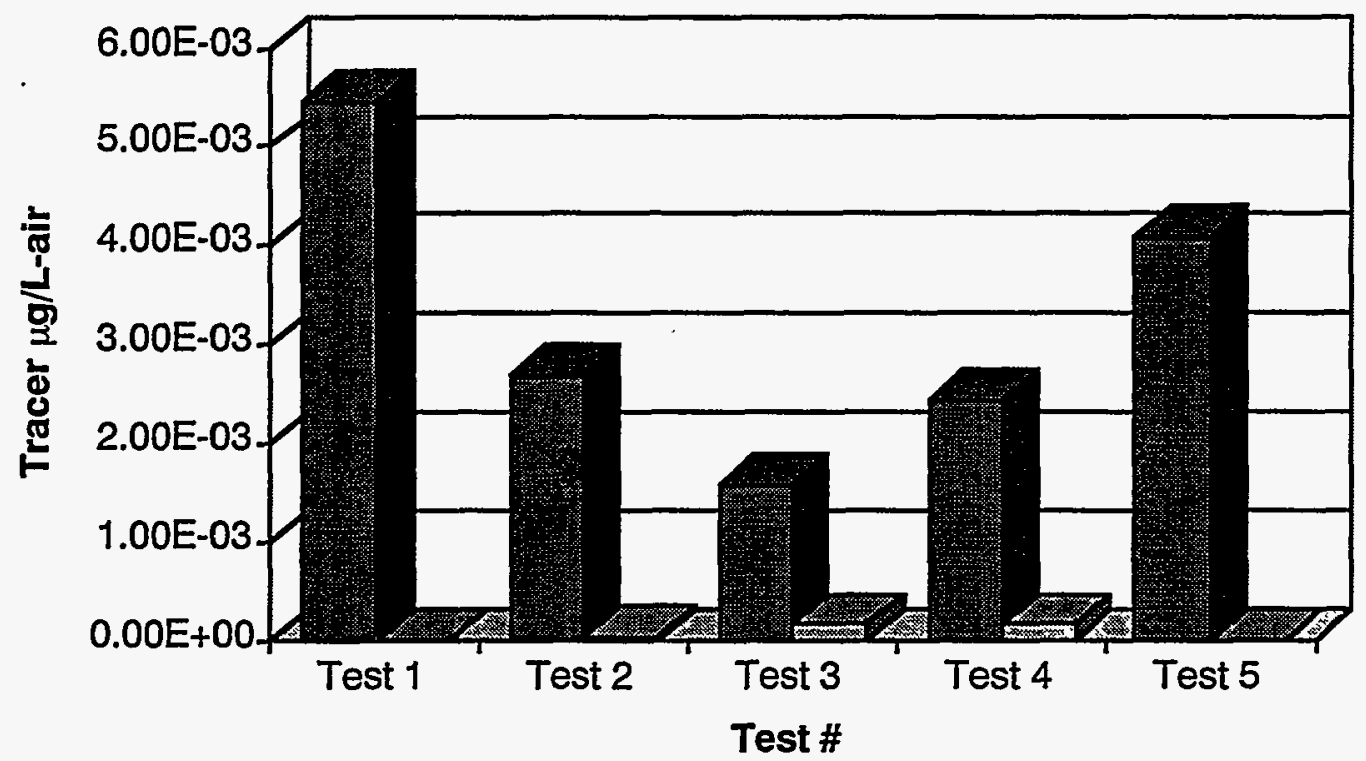

Test Mean

图 Background Mean

Figure F-14. Test means for the five rare earth tests.

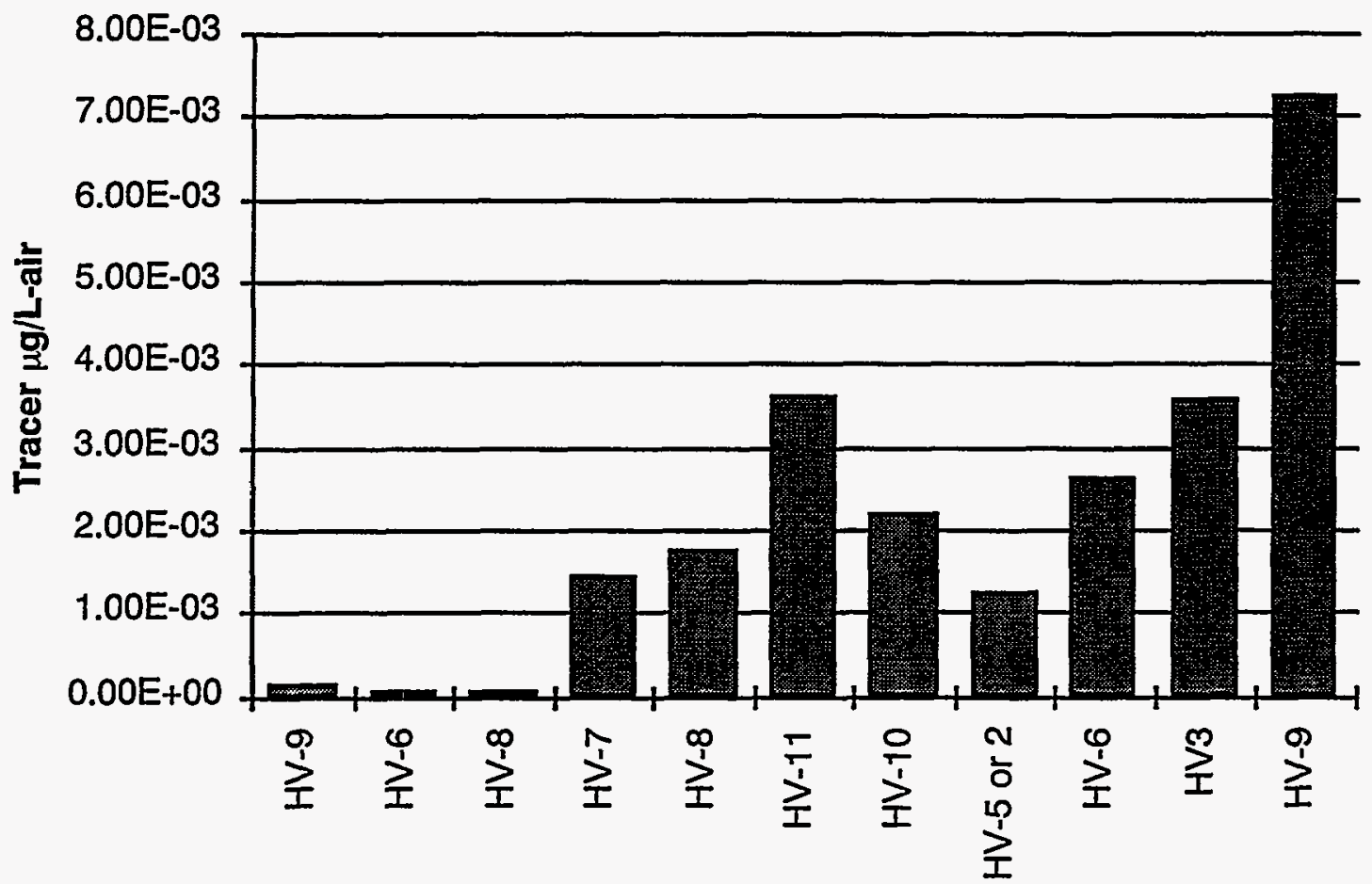

Monitor Number

Figure F-15. The average tracer collected for each air monitor during the demonstration clearly shows that HV-9, the monitor closest to the digging, had the highest average tracer loading. This 
supports the visual observations that virtually all of the dust generation was a result of the digging operations, and that the dumping operations (or placement of the ITM) created virtually no dust.

The purpose of performing rare earth tracer analysis has traditionally been to confirm the source of potential contamination spread (dust). An interesting finding in this demonstration is the almost perfect correlation ( 0.98 with 1.0 being perfect) between dust and rare earth tracer spread (see Figure F-16). Intuitively this should be obvious, however because of difficulty in controlling dust sources in the past, it has been deemed necessary to perform the rare earth tracer study. The implications of this are that if performing a demonstration inside a structure that sufficiently controls the environment, and only a single source of potential dust exists, then the rare earth tracer study need not be performed. This can lead to a tremendous cost savings, as the ICP-MS analysis of the filters for rare earths is very expensive.

\section{Dust and Rare Earth Tracer Correlation}

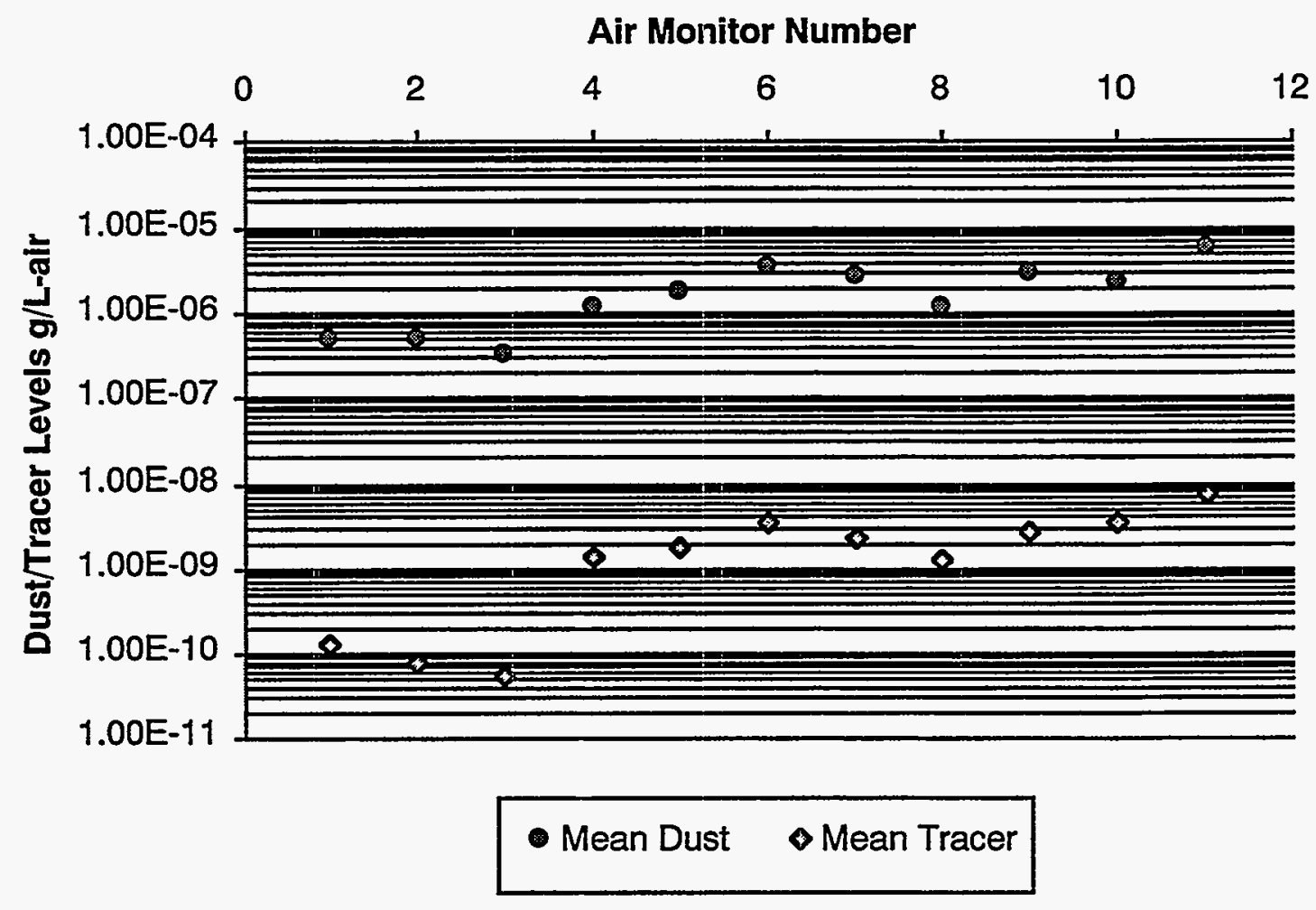

Figure F-16. There is a very high correlation between dust and rare earth tracer collected on each air monitor. Note that the curves for the mean dust and the mean tracer collected on each air monitor (averaged across tests) match very closely. 


\section{F-3. SOIL MOISTURE DATA}

The raw data is contained in Table F-13. The method of determining soil moisture content occurred as follows. The soils were first weight, dried overnight at $105^{\circ} \mathrm{C}$, cooled in a desiccator then reweighed and the moisture content was then calculated.

Table F-13. Soil moisture.

\begin{tabular}{cc}
\hline Field sample number & Total \% moisture \\
\hline Test 1 Initial & 5.90 \\
Test 1 Post & 9.00 \\
Test 2 Pre & 8.50 \\
Test 2 Post & 9.10 \\
Test 3 Pre & 8.80 \\
Test 4 Post & 7.60 \\
Test 5 Pre & 6.50 \\
Test 5 Post & 10.40 \\
\hline
\end{tabular}

The average moisture content for the demonstration program is $8.23 \%$ for each of the samples with a standard deviation of 1.48. Although the natural soil moisture is approximately $18 \%$ under normal dry conditions, a worst case (driest) soil moisture content was determined to be 4 to $8 \%$. Correlations were calculated and plotted between the soil moisture content and the average amount of dust and rare earth tracer measured on the filters for each test. The correlation coefficient for soil moisture vs. dust was -0.98 (significant for a levels of 0.05 and 0.01 ), and for soil moisture vs. tracer was -0.66 (not significant) indicating on the surface that a strong correlation exists between soil moisture and dust generation. Intuitively this should be correct and probably is; the more soil moisture, the less dust. This should also be true of tracer, but the data does not bear this out. This is probably due to the lack of a sufficient number of data points, and variability across the data. 


\section{F-4. CALIBRATION DATA}

Figure F-17 provides a plot of the data for correlation purposes bases on the raw data contained in Table F-14 for the calibration data of the high volume air samples. The data was corrected to standard temperature and pressure at 29.92 in $\mathrm{Hg}$ and $70^{\circ} \mathrm{C}$. The data are relatively consistent between each of the high volume air samples. The air flow calculations for the test data was extrapolated from the raw data utilizing a polynomial curve fit equation.

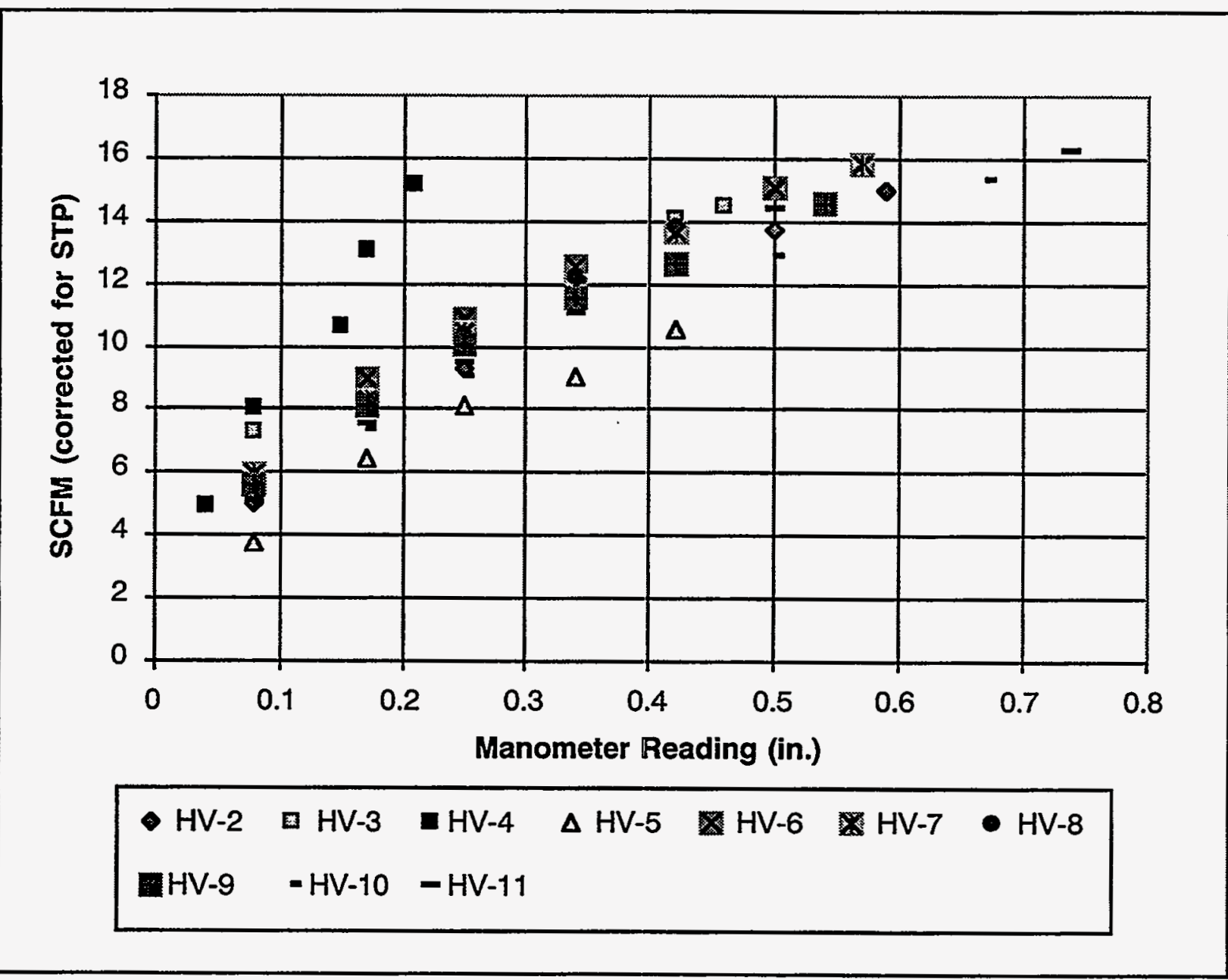

Figure F-17. High volume air samples (corrected to standard temperature and pressure). 
Table F-14. Calibration data for high volume air samplers (corrected to standard temperature and pressure).

\begin{tabular}{|c|c|c|c|c|c|c|c|c|c|c|}
\hline Manometer & HV-2 & $\mathrm{HV}-3$ & $\mathrm{HV}-4$ & HV-5 & HV-6 & $\mathrm{HV}-7$ & HV-8 & HV-9 & HV-10 & $\mathrm{HV}-11$ \\
\hline $\begin{array}{l}0.04 \\
0.08\end{array}$ & 499 & 7.29 & $\begin{array}{l}4.98 \\
8.03\end{array}$ & 3.77 & 500 & 508 & 554 & 562 & $50 ?$ & 5.12 \\
\hline 0.15 & & & 10.63 & & & & & & & \\
\hline 0.17 & & 8.31 & 13.05 & 6.48 & 8.96 & 8.26 & 7.98 & 8.15 & 7.37 & 7.51 \\
\hline 0.21 & & & 15.15 & & & & & & & \\
\hline 0.25 & 9.30 & 10.78 & & 8.16 & 10.87 & 10.43 & 10.39 & 10.04 & 9.01 & 9.50 \\
\hline 0.34 & & 12.45 & & 9.02 & 12.52 & 11.94 & 12.20 & 11.58 & 12.09 & 11.05 \\
\hline 0.42 & 12.63 & 14.05 & & 10.54 & & 13.62 & 13.78 & 12.66 & & \\
\hline 0.46 & & 14.45 & & & & & & & & \\
\hline 0.50 & 13.72 & & & & 15.11 & & & & 12.90 & 14.44 \\
\hline 0.54 & & & & & & & & 14.54 & & \\
\hline 0.57 & & & & & & 15.81 & & & & \\
\hline 0.59 & 14.95 & & & & & & & & & \\
\hline 0.67 & & & & & & & & & 15.36 & \\
\hline 0.74 & & & & & & & & & & 16.24 \\
\hline
\end{tabular}




\section{F-5. RAW TEST DATA}

The following raw data is provided in tabulated form for each of the tests. The data contains the sample number, pre-weight and post-weights, the HV-\# utilized during the testing, the calculated g/L-air for both dust and tracer tests. Each UUT or HV manometer was corrected to standard temperatures and pressures before final calculation. 
Table F-15. Test 1 data.

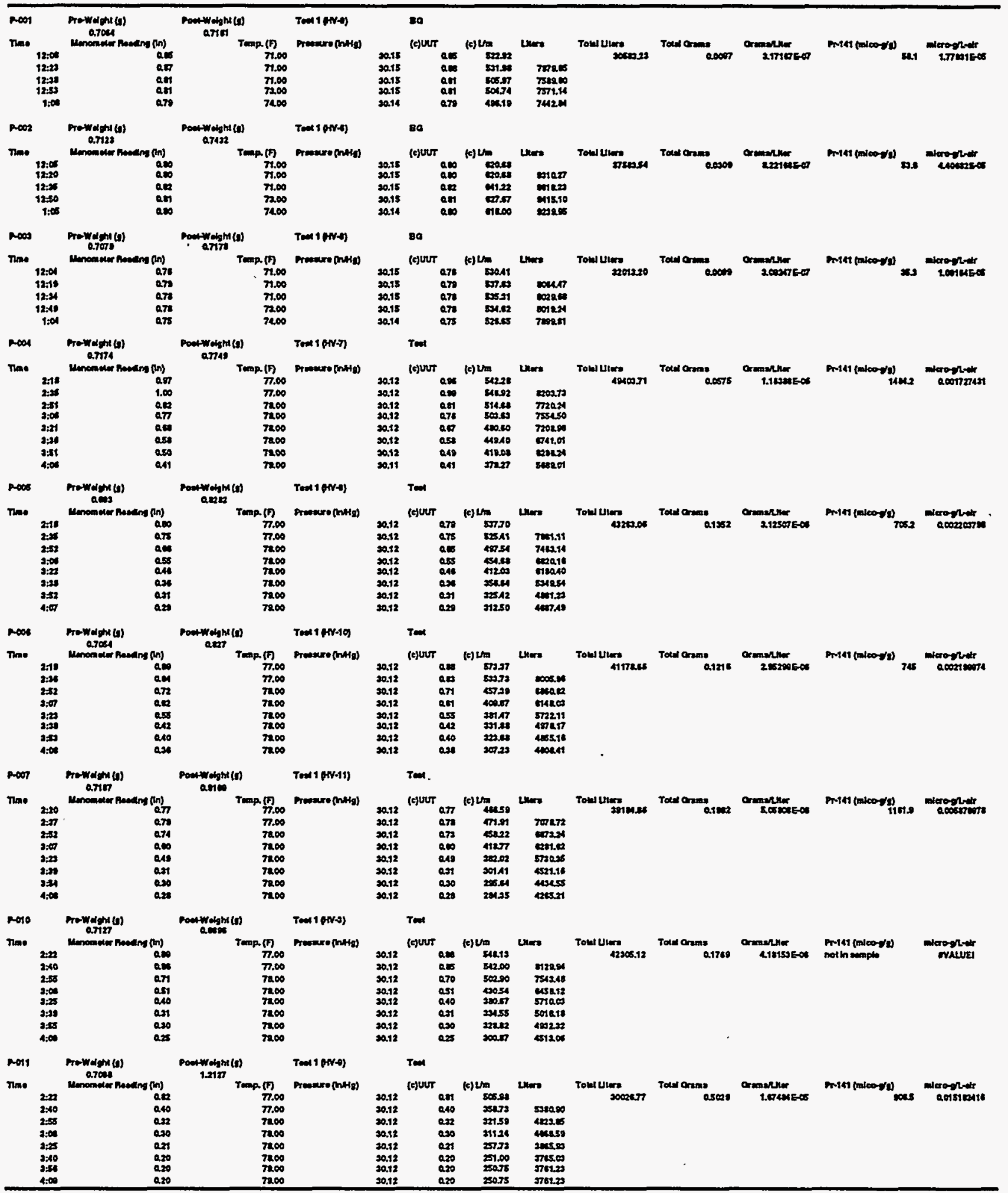


Table F-16. Test 2 data.

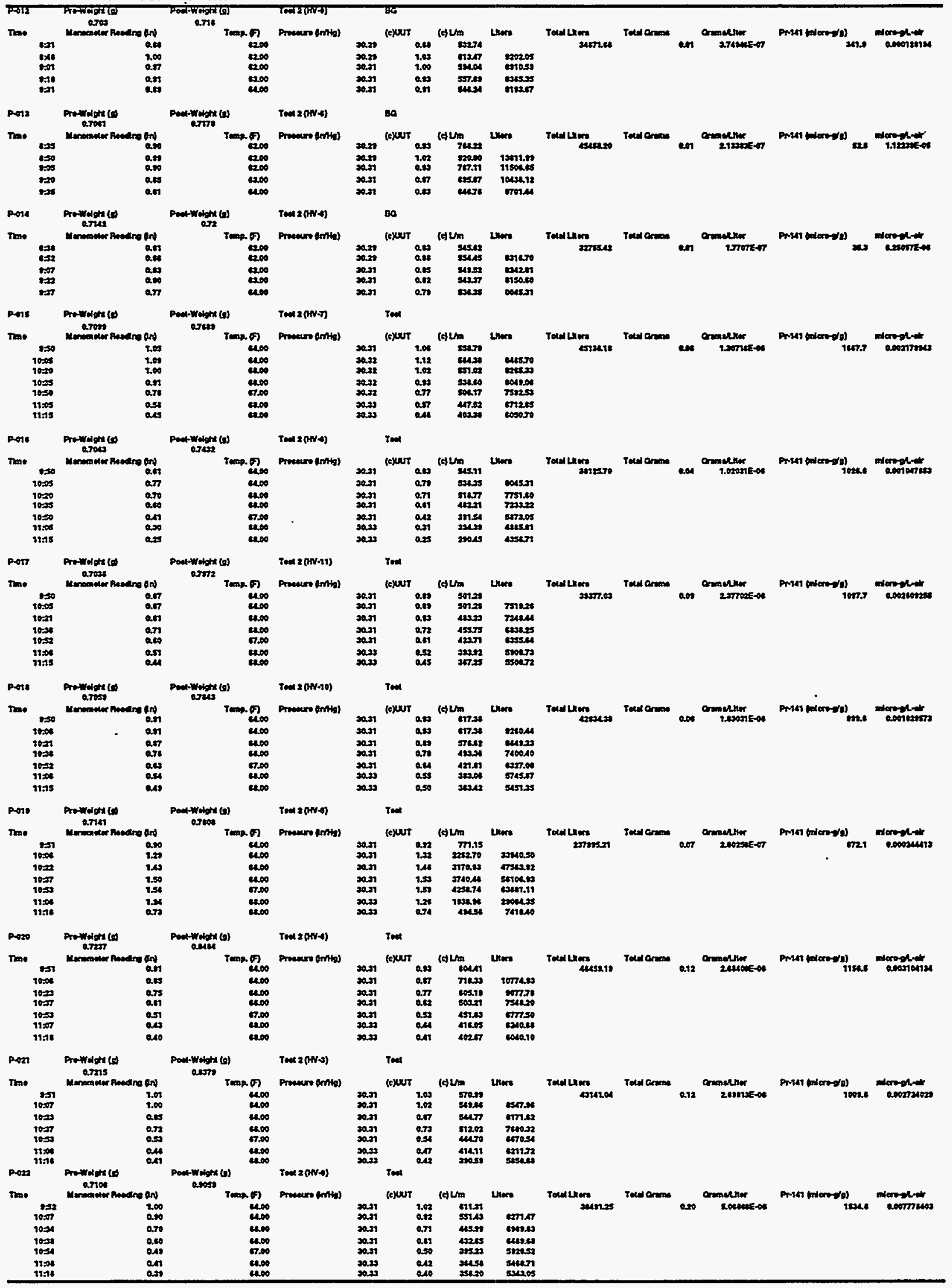


Table F-17. Test 3 data.

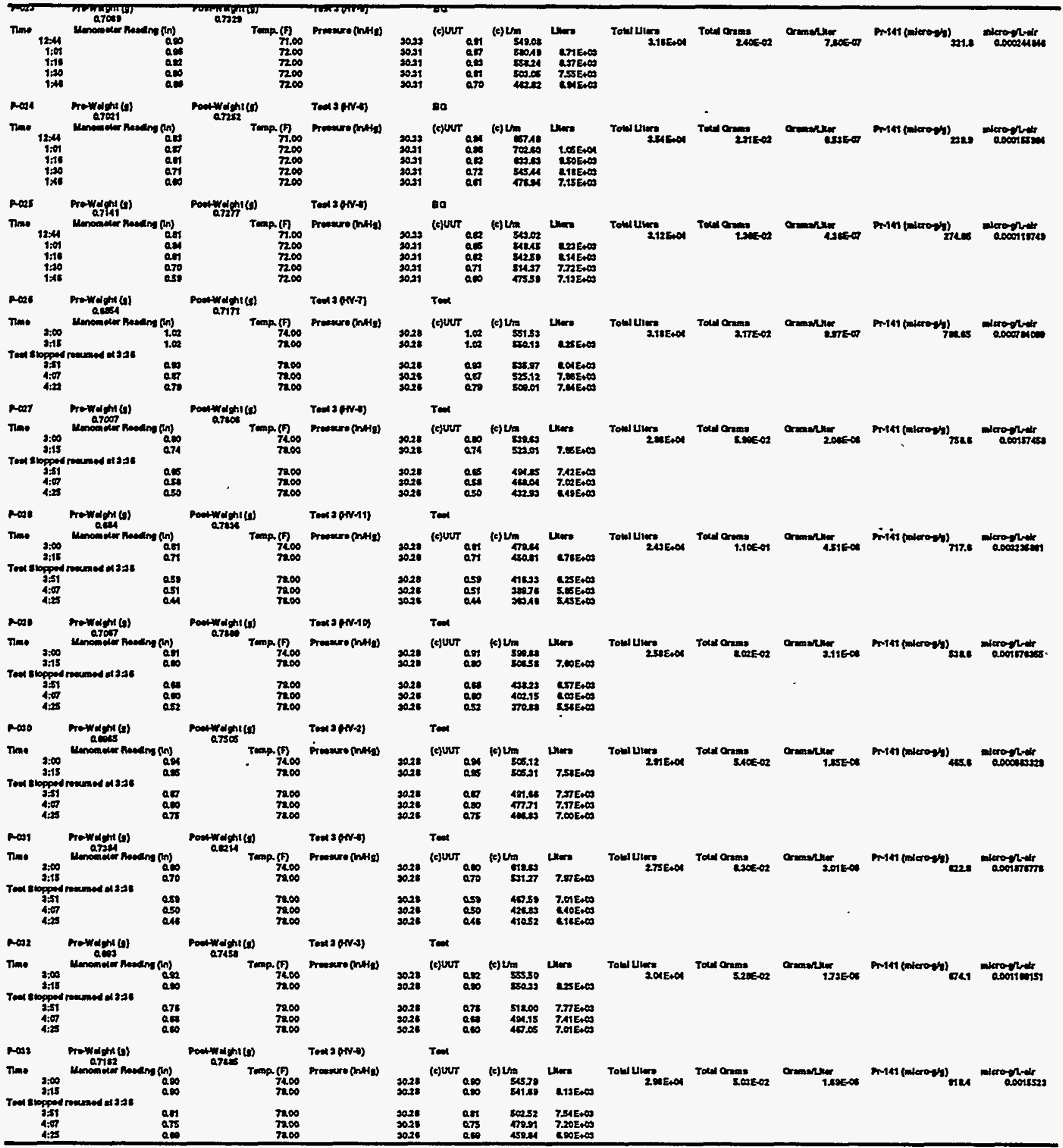




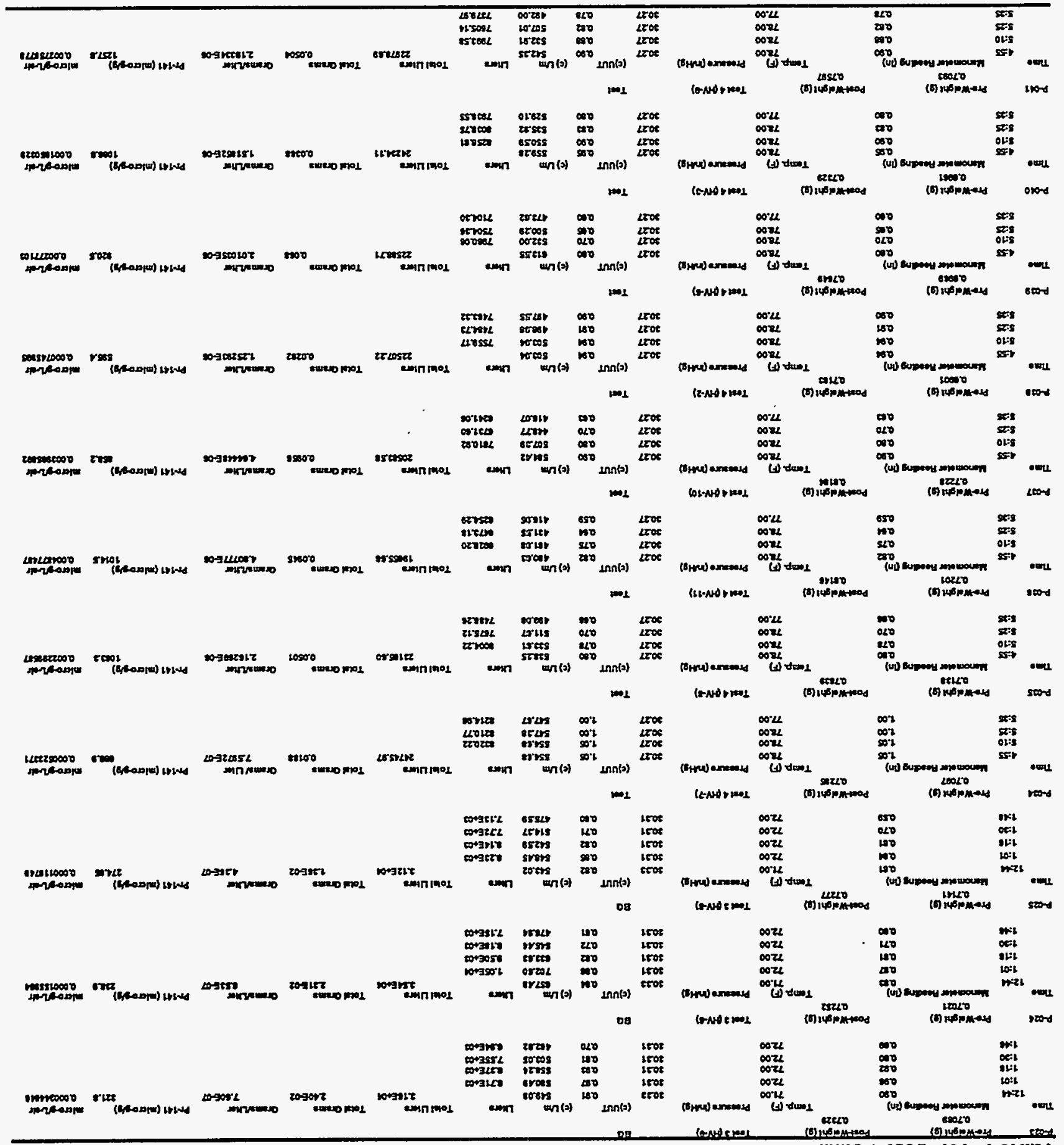


Table F-19. Test 5 data.

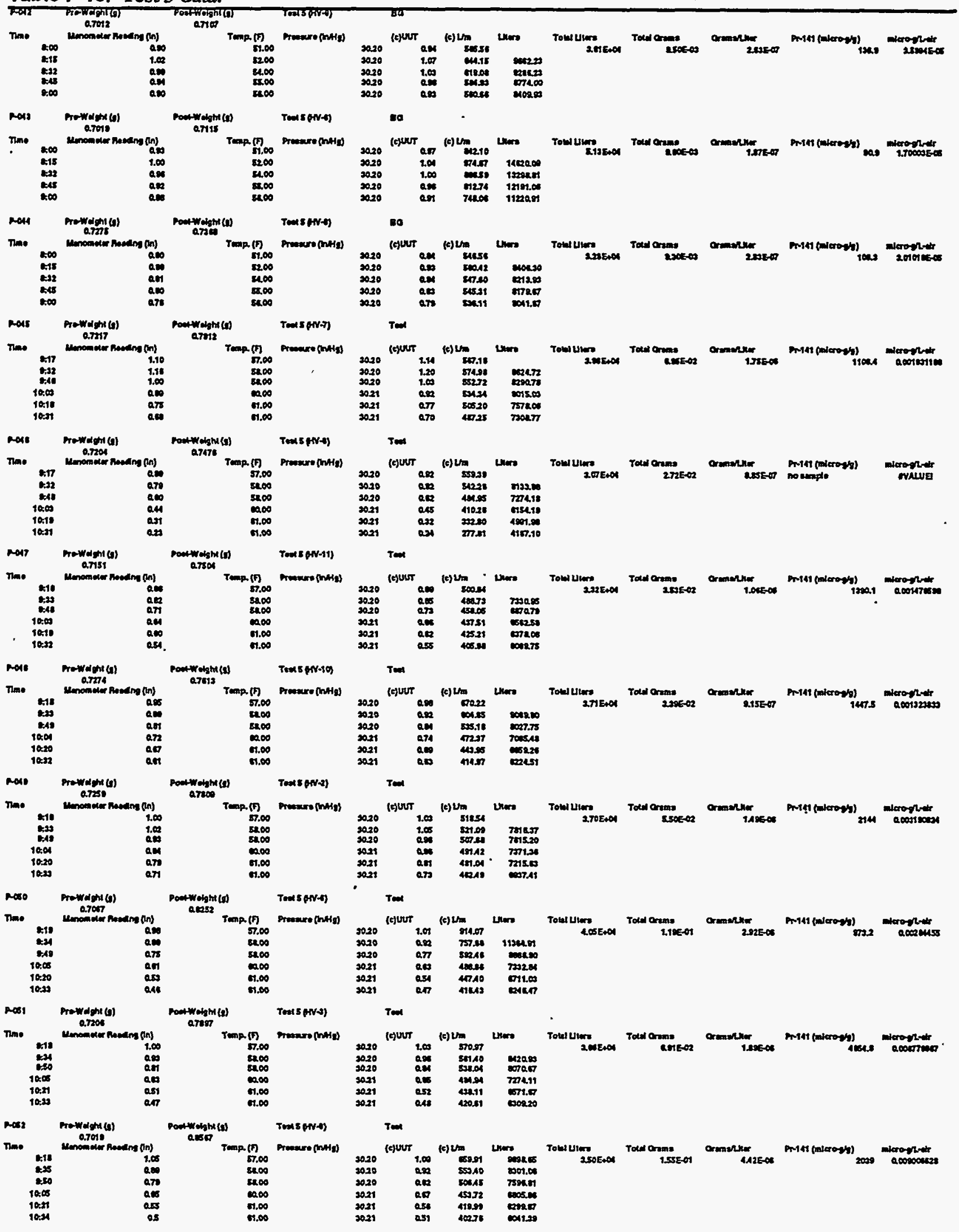




\section{Appendix G \\ Phase I Detailed Test Data}



Table G-1. Test bed waste form volumes.

\begin{tabular}{||l|c|c|c||}
\hline \multicolumn{3}{|c|}{ Test Bed Waste Form Volumes } \\
\hline \multicolumn{1}{||c|}{ Waste Type } & $\begin{array}{c}\text { Volume } \\
\left(\mathbf{y d}^{3}\right)\end{array}$ & Quantity & $\begin{array}{c}\text { Total Volume } \\
\left(\mathbf{y d}^{3}\right)\end{array}$ \\
\hline INEL Dirt & 40.00 & & 40.00 \\
\hline Paper & 12.00 & & 12.00 \\
\hline $\begin{array}{l}\text { 55 Gal. Drums Barrel (filled } \\
\text { with dry Portland cement) }\end{array}$ & 00.27 & 18.0 & 4.86 \\
\hline Cement Rounds & 00.08 & 20.0 & 1.60 \\
\hline 3' Large Steel Beam & 00.10 & 15.0 & 1.50 \\
\hline 7' Large Steel Beams & 0.24 & 1.0 & 0.24 \\
\hline \multicolumn{1}{|c|}{ TOTAL } & & & 60.20 \\
\hline
\end{tabular}

Table G-2. Phase I testing-Test \#1.

\begin{tabular}{|c|c|c|c|c|}
\hline & \multicolumn{4}{|c|}{$\begin{array}{c}\text { PHASE I TESTING - TEST \#1 } \\
\text { (Test Date 9-20-94, Test Time 2:30 p.m. - 4:06 p.m.) }\end{array}$} \\
\hline Cycle & $\begin{array}{c}\text { Dig \& Release } \\
\text { Cycle Time } \\
\text { (min.) }\end{array}$ & $\begin{array}{c}\text { Type \& Amount of Removed } \\
\text { Objects }\end{array}$ & $\begin{array}{l}\text { Volume in } \\
\text { ITM } \\
\left(\mathrm{yd}^{3}\right)\end{array}$ & $\begin{array}{c}\text { Volume } \\
\text { Spilled } \\
\left(y d^{3}\right)\end{array}$ \\
\hline 1 & 7 & 1/16 Dirt & 0.09 & .0 \\
\hline 2 & 6 & 1/2 Dirt & 0.70 & .0 \\
\hline 3 & 3 & 1 Barrel & 0.27 & .0 \\
\hline 4 & 6 & 1 Barrel \& 1/16 Dirt & 0.36 & .0 \\
\hline 5 & 11 & 2 Barrels & 0.54 & .0 \\
\hline 6 & 5 & 1 Barrel \& 1/4 Dirt & 0.62 & .0 \\
\hline 7 & 9 & $7^{\prime}$ Beam & 0.24 & .0 \\
\hline 8 & 10 & 7/8 Dirt & 1.22 & .0 \\
\hline TOTAL & $57 \mathrm{~min}$. & & $4.04 \mathrm{yd}^{3}$ & .0 \\
\hline
\end{tabular}


Table G-3. Phase I testing-Test \#2.

\begin{tabular}{|c|c|c|c|c|}
\hline & \multicolumn{4}{|c|}{$\begin{array}{l}\text { PHASE I TESTING - TEST \#2 } \\
\text { (Test Date 9-21-94, Test Time 10:00 a.m. - 11:09 a.m.) }\end{array}$} \\
\hline Cycle & $\begin{array}{c}\text { Dig \& Release } \\
\text { Cycle Time } \\
\text { (min) }\end{array}$ & $\begin{array}{l}\text { Type \& Amount of } \\
\text { Objects Removed }\end{array}$ & $\begin{array}{c}\text { Volume } \\
\text { in ITM } \\
\left(\mathrm{yd}^{3}\right)\end{array}$ & $\begin{array}{c}\text { Volume } \\
\text { Spilled } \\
(\mathrm{yd} 3)\end{array}$ \\
\hline 1 & 3 & 7/8 Dirt & 1.22 & Light Dust \\
\hline 2 & 3 & 1/2 Dirt \& Paper & 0.70 & Light Dust \\
\hline 3 & 2 & 1/2 Dirt \& 3' Beam & 0.80 & Light Dust \\
\hline 4 & 3 & 1/2 Dirt & 0.70 & Light Dust \\
\hline 5 & 5 & 1 Barrel & 0.27 & Light Dust \\
\hline 6 & 5 & 1 Barrel & 0.27 & Light Dust \\
\hline 7 & 4 & 1 Barrel (Paper, Dirt) & 0.27 & Light Dust \\
\hline 8 & 3 & 3/4 Dirt \& Paper & 1.05 & Light Dust \\
\hline 9 & 7 & 1 Barrel \& Paper & 0.27 & Light Dust \\
\hline 10 & 5 & 1 Barrel & 0.27 & Light Dust \\
\hline 11 & 5 & 1 Barrel \& 1 Cement Round & 0.35 & Light Dust \\
\hline 12 & 6 & 1/2 Dirt & 0.70 & Light Dust \\
\hline 13 & 5 & Full \& Heaped with Paper & 1.40 & Light Dust \\
\hline$* 14$ & not timed & not recorded & 0 & Light Dust \\
\hline TOTAL & $56 \mathrm{~min}$. & & $8.27 \mathrm{yd}^{3}$ & $\begin{array}{c}2 \text { cups of dirt } \\
0.0006 \mathrm{yd}^{3}\end{array}$ \\
\hline & Removal R & $8.27 \mathrm{yd}^{3} \times 1 / 56 \min \times 6$ & $=8$ & \\
\hline
\end{tabular}


Table G-4. Phase I testing-Test \#3.

\begin{tabular}{|c|c|c|c|c|}
\hline & \multicolumn{4}{|c|}{$\begin{array}{c}\text { PHASE I TESTING - TEST \#3 } \\
\text { (Test Date 9/21/94, Test Time 3:05 p.m. - 4:22 p.m.) }\end{array}$} \\
\hline Cycle & $\begin{array}{l}\text { Dig \& Release } \\
\text { Cycle Time } \\
\text { (min) }\end{array}$ & $\begin{array}{l}\text { Type \& Amount of } \\
\text { Objects Removed }\end{array}$ & $\begin{array}{l}\text { Volume in } \\
\text { ITM } \\
\left(\mathrm{yd}^{3}\right)\end{array}$ & $\begin{array}{l}\text { Volume } \\
\text { Spilled } \\
\left(\mathrm{yd}^{3}\right)\end{array}$ \\
\hline 1 & 2 & 1/2 Dirt \& Paper & 0.70 & Light Dust \\
\hline 2 & 7 & 1 Barrel \& $1 / 2$ Dirt & 0.97 & Light Dust \\
\hline 3 & 7 & $\begin{array}{l}\text { 3/4 Dirt } \\
\text { \& Paper }\end{array}$ & 1.05 & $\begin{array}{l}\text { Light Dust } \\
\text { \& Paper }\end{array}$ \\
\hline 4 & 7 & 1 Barrel \& 1/4 Dirt & 0.62 & Light Dust \\
\hline 5 & 9 & 1 Cement Round \& 1/2 Dirt & 0.78 & Light Dust \\
\hline 6 & 5 & $\begin{array}{c}1 \text { Barrel, } 13^{\prime} \text { Beam \& } 1 / 2 \\
\text { Dirt }\end{array}$ & 1.07 & Light Dust \\
\hline 7 & 4 & 1 Cement Round \& 3/4 Dirt & 1.13 & Light Dust \\
\hline 8 & 2 & $\begin{array}{l}\text { 3/4 Dirt } \\
\text { \& Paper }\end{array}$ & 1.05 & Light Dust \& Paper \\
\hline 9 & - & $\begin{array}{l}\text { 7/8 Dirt } \\
\text { \& Paper }\end{array}$ & 1.22 & Light Dust \\
\hline 10 & 4.0 & $\begin{array}{l}\text { 1/2 Dirt } \\
\text { \& Paper }\end{array}$ & 0.7 & Light Dust \\
\hline TOTAL & $\overline{47}$ & & 9.29 & $\begin{array}{c}1 / 2 \text { Cup Dirt \& } 1 / 2 \\
\text { Paper or } \\
0.00015 \mathrm{yd}^{3} \\
\end{array}$ \\
\hline \multicolumn{5}{|c|}{ *Cycle \#9 not included in Remove Rate Calculations } \\
\hline & Removal Ra & $8.07 \mathrm{yd}^{3} \times 1 / 47 \min \times 60$ & $\mathrm{r}=\mathbf{1 0 . 3}$ & \\
\hline
\end{tabular}


Table G-5. Phase I testing-Test \#4.

\begin{tabular}{|c|c|c|c|c|}
\hline & \multicolumn{4}{|c|}{$\begin{array}{c}\text { PHASE I TESTING - TEST \#4 } \\
\text { (Test Date 9-21-94, Test Time 5:00 - 5:34 p.m.) }\end{array}$} \\
\hline Cycle & $\begin{array}{l}\text { Dig \& Release } \\
\text { Cycle Time }\end{array}$ & $\begin{array}{l}\text { Type \& Amount of } \\
\text { Objects Removed }\end{array}$ & $\begin{array}{c}\text { Volume In } \\
\text { ITM } \\
\left(\mathrm{yd}^{3}\right) \\
\end{array}$ & $\begin{array}{c}\text { Volume } \\
\text { Spilled } \\
\left(\mathrm{yd}^{3}\right)\end{array}$ \\
\hline 1 & 3 & 1 Cement \& 3/4 Dirt & 1.13 & Light Dust \\
\hline 2 & 2 & 1/2 Dirt \& Paper & .7 & Light Dust \\
\hline 3 & 2 & 1/2 Dirt \& Paper & .7 & Light Dust \\
\hline$\overline{4}$ & 3 & $\begin{array}{l}1 \text { Cement Round \& Full } \\
\text { Dirt }\end{array}$ & 1.48 & Light Dust \\
\hline 5 & 2 & 7/8 Dirt \& Paper & 1.22 & Light Dust \\
\hline 6 & 5 & 7/8 Dirt \& Paper & 1.22 & Light Dust \\
\hline 7 & 2 & 1 Cement Round \& 3/4 Dirt & 1.13 & Light Dust \\
\hline 8 & 3 & 1/2 Dirt \& Paper & .7 & Light Dust \\
\hline 9 & 2 & 3/4 Dirt \& Paper & 1.05 & Light Dust \\
\hline 10 & 3 & 3/4 Dirt \& Paper & 1.05 & Light Dust \\
\hline TOTAL & 27 & & 10.38 & $\begin{array}{c}6 \text { Cups Dirt } \\
\& 1 / 4 \text { Paper or } \\
0.0018 \text { yd }^{3} \\
\end{array}$ \\
\hline \multicolumn{5}{|c|}{ Removal Rate $=10.38 \mathrm{yd}^{3} \times 1 / 27 \mathrm{~min} \times 60 \mathrm{~min} / \mathrm{hr}=23.1 \mathrm{yd}^{3} / \mathrm{hr}$} \\
\hline
\end{tabular}


Table G-6. Phase I testing-Test \#5.

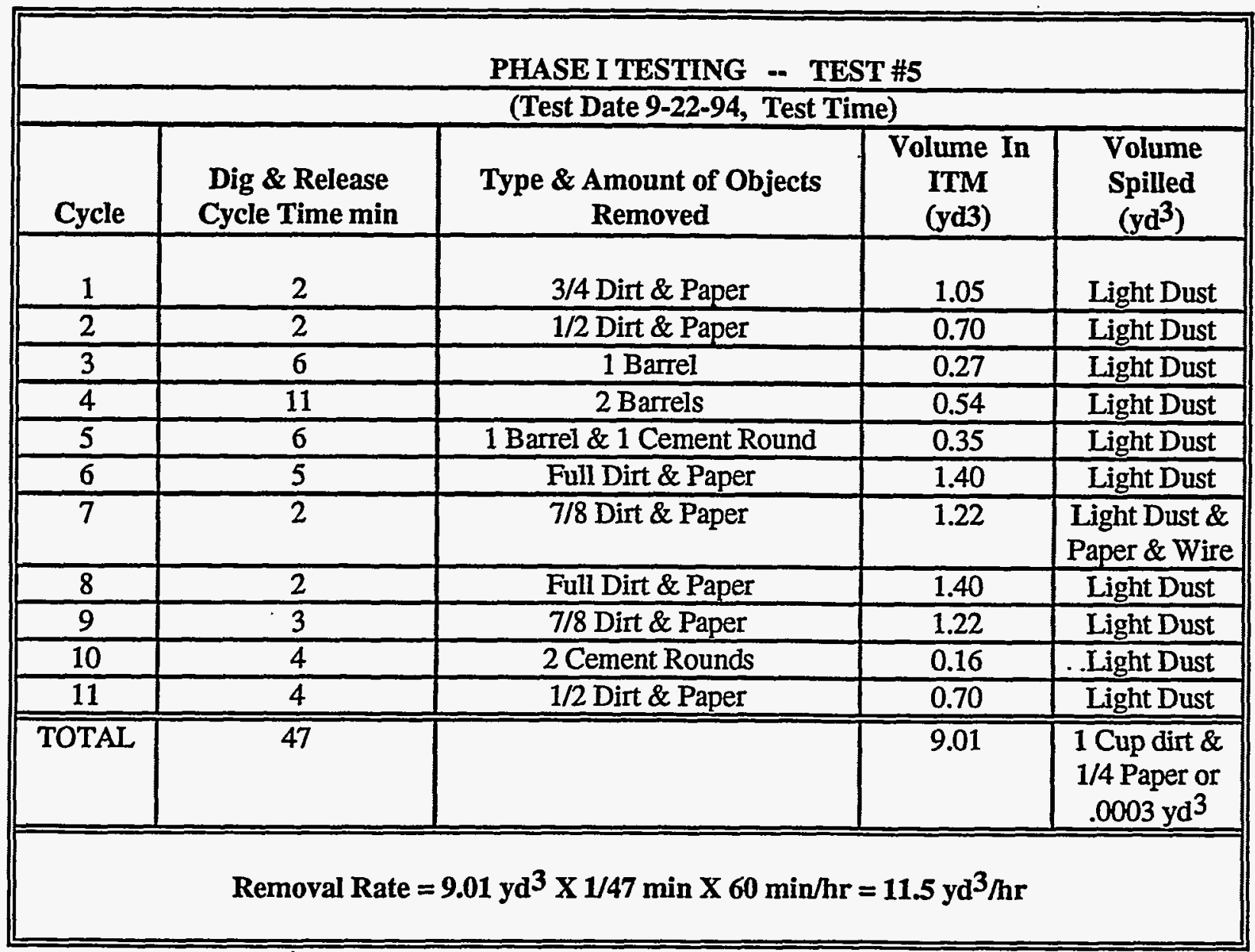




\section{Appendix $\mathrm{H}$}

\section{Phase II Detailed Test Data}


Table H-1.

\begin{tabular}{|c|c|c|c|c|c|}
\hline \multicolumn{6}{|c|}{ DATE: $9.27-94$} \\
\hline Cycle & $\begin{array}{c}\text { Excavator } \\
\text { Cycle Time } \\
\text { (min) }\end{array}$ & $\begin{array}{c}\text { Conveyance } \\
\text { Cycle Time } \\
\text { (min) }\end{array}$ & $\begin{array}{c}\text { Total } \\
\text { Cycle Time } \\
\text { (min) }\end{array}$ & $\begin{array}{l}\text { Type \& Amount of } \\
\text { Removed Objects }\end{array}$ & $\begin{array}{c}\text { Volume } \\
\text { in ITM } \\
\left(\mathrm{yd}^{\mathbf{3}}\right)\end{array}$ \\
\hline 1 & $2: 29$ & $16: 37$ & 19:06 & $\begin{array}{l}\text { 7/8 Dirt \& Paper \& Barrel, 3' } \\
\text { Beam }\end{array}$ & 1.22 \\
\hline
\end{tabular}

Table H-2.

\begin{tabular}{|c|c|c|c|c|c|}
\hline \multicolumn{6}{|c|}{ DATE: 9-28-94 } \\
\hline Cycle & $\begin{array}{l}\text { Excavator } \\
\text { Cycle Time } \\
\text { (min) }\end{array}$ & $\begin{array}{c}\text { Conveyance } \\
\text { Cycle .Time } \\
\text { (min) }\end{array}$ & $\begin{array}{c}\text { Total } \\
\text { Cycle Time } \\
\text { (min) }\end{array}$ & $\begin{array}{l}\text { Type \& Amount of } \\
\text { Removed Objects }\end{array}$ & $\begin{array}{c}\text { Volume } \\
\text { in ITM } \\
\left(\mathrm{yd}^{3}\right)\end{array}$ \\
\hline 1 & $6: 32$ & $11: 46$ & $18: 18$ & 7/8 Paper \& Dirt \& Barrel & 1.22 \\
\hline 2 & $4: 12$ & 12:09 & $16: 21$ & $\begin{array}{l}\text { 1/2 Paper \& Dirt, Cement } \\
\text { Round, Barrel }\end{array}$ & 0.70 \\
\hline 3 & $4: 36$ & $11: 37$ & $16: 13$ & 1/2 Paper \& Dirt \& Barrel & 0.70 \\
\hline 4 & $3: 34$ & $11: 36$ & $15: 10$ & 3/4 Paper \& Dirt \& 2 Barrels & 1.05 \\
\hline 5 & $3: 10$ & $10: 45$ & $13: 55$ & $\begin{array}{l}\text { 1/2 Paper \& Two 50' Lengths } \\
\text { of Cable }\end{array}$ & 0.70 \\
\hline 6 & -- & $10: 09$ & -- & 1/2 Paper \& Dirt \& Wood & 0.70 \\
\hline 7 & $3: 06$ & $10: 02$ & $13: 08$ & $\begin{array}{l}\text { Full Paper \& Dirt, Barrel \& } \\
\text { Cement Round }\end{array}$ & 1.40 \\
\hline 8 & $3: 56$ & $10: 15$ & $14: 11$ & Full Paper \& Dirt \& 2 Barrels & 1.40 \\
\hline 9 & $3: 54$ & $8: 38$ & $12: 32$ & 7/8 Paper \& Dirt \& 3' Beam & 1.05 \\
\hline 10 & $2: 57$ & $8: 44$ & $11: 41$ & $\begin{array}{l}\text { Full Paper \& Dirt, Barrel, } 31 \\
\text { Beam }\end{array}$ & 1.40 \\
\hline 11 & $2: 49$ & $8: 23$ & $11: 12$ & $\begin{array}{l}\text { Full Paper \& Dirt, Barrel, } \\
\text { Cement Round }\end{array}$ & 1.40 \\
\hline 12 & $3: 10$ & $10: 26$ & $13: 36$ & Full Paper \& Dirt, Barrel & 1.40 \\
\hline 13 & $2: 59$ & $8: 55$ & $11: 54$ & Full Paper \& Dirt, Barrel & $\overline{1.4}$ \\
\hline 14 & $\overline{---}$ & $8: 57$ & $\overline{-\cdots}$ & $\begin{array}{l}\text { Full (heaped) Paper \& Dirt, } 3^{\prime} \\
\text { Beam }\end{array}$ & 1.40 \\
\hline 15 & $3: 39$ & $10: 50$ & $14: 29$ & $\begin{array}{l}\text { Full (heaped) Paper \& Dirt, } \\
\text { Barrel, } 3 \text { Cement Rounds }\end{array}$ & 1.40 \\
\hline 16 & $3: 46$ & $8: 35$ & $12: 21$ & $\begin{array}{l}\text { Full Paper \& Dirt, } 2 \text { Cement } \\
\text { Rounds, 3' Beam }\end{array}$ & 1.40 \\
\hline 17 & $3: 04$ & $10: 10$ & $13: 14$ & Full Paper \& Dirt & 1.40 \\
\hline 18 & $3: 06$ & $10: 23$ & $13: 29$ & $\begin{array}{l}\text { 7/8 Paper \& Dirt, Cement } \\
\text { Round }\end{array}$ & 1.22 \\
\hline 19 & $\overline{-\cdots}$ & $9: 42$ & $\overline{-\cdots}$ & $\begin{array}{l}\text { Filled WTC with Truck Bed } \\
\& 2 \text { Barrels }\end{array}$ & 0 \\
\hline
\end{tabular}


Table H-3.

\begin{tabular}{|c|c|c|c|c|c|}
\hline \multicolumn{6}{|c|}{ DATE: 9-29-94 } \\
\hline Cycle & $\begin{array}{c}\text { Excavator } \\
\text { Cycle Time } \\
\text { (min) }\end{array}$ & $\begin{array}{l}\text { Conveyance } \\
\text { Cycle Time } \\
\text { (min) }\end{array}$ & $\begin{array}{c}\text { Total } \\
\text { Cycle Time } \\
\text { (min) }\end{array}$ & $\begin{array}{c}\text { Type \& Amount } \\
\text { of Removed Objects }\end{array}$ & $\begin{array}{c}\text { Volume } \\
\text { in ITM } \\
\left(\mathrm{yd}^{3}\right)\end{array}$ \\
\hline$\overline{1}$ & $2: 33$ & $\cdots$ & $\overline{-\infty}$ & $\begin{array}{l}\text { Full Paper \& Dirt, Wood, } \\
\text { Cement Round }\end{array}$ & 1.40 \\
\hline 2 & $3: 15$ & $8: 16$ & $11: 31$ & 1/2 Paper \& Dirt, Barrel & 0.70 \\
\hline 3 & $3: 31$ & $8: 35$ & $12: 06$ & $\begin{array}{l}\text { 2/3 Paper \& Dirt, Wire, } \\
\text { Barrel }\end{array}$ & 0.93 \\
\hline 4 & $2: 38$ & $10: 19$ & $12 ; 57$ & 7/8 Paper \& Dirt & 1.22 \\
\hline 5 & $3: 29$ & $8: 30$ & $11: 59$ & 1/2 Paper \& Dirt & 0.70 \\
\hline 6 & $2: 50$ & $8: 34$ & $11: 24$ & $\begin{array}{l}\text { 3/4 Paper \& Dirt, } 2 \times 4 \times 6 \\
\text { Wood }\end{array}$ & 1.05 \\
\hline$\overline{7}$ & $2: 37$ & $8: 20$ & $10: 57$ & $\begin{array}{l}\text { 3/4 Paper \& Dirt, 3' Beam, } \\
\text { Cement Round }\end{array}$ & 1.05 \\
\hline 8 & $2: 37$ & $8: 45$ & $11: 22$ & Full Paper \& Dirt & 1.40 \\
\hline 9 & $2: 16$ & $8: 57$ & $11: 13$ & Full Paper \& Dirt, 3' Beam & 1.40 \\
\hline 10 & $2: 30$ & $8: 24$ & $10: 54$ & $\begin{array}{l}\text { 3/4 Paper \& Dirt } 3 \text { Beam, } \\
\text { Cement Round }\end{array}$ & 1.05 \\
\hline 11 & $2: 16$ & $8: 21$ & $10: 37$ & 3/4 Paper \& Dirt & 1.05 \\
\hline 12 & $3: 36$ & $8: 21$ & $11: 57$ & Full Paper \& Dirt & 1.40 \\
\hline 13 & $4: 17$ & $8: 04$ & $12: 21$ & 4/3 Paper \& Dirt & 1.05 \\
\hline 14 & 3:09 & $-\cdots$ & $-\cdots$ & 1/2 Paper \& Dirt, Barrel & 0.70 \\
\hline 15 & $5: 37$ & $8: 28$ & $14: 05$ & Full Paper \& Dirt, Barrel & 1.40 \\
\hline 16 & $2: 55$ & 16:01 & $18: 56$ & Full Paper \& Dirt, 2 Barrels & 1.40 \\
\hline 17 & $2: 43$ & 9:25 & $12: 08$ & 3/4 Paper \& Dirt & 1.05 \\
\hline 18 & $2: 35$ & $8: 18$ & $10: 53$ & Full Dirt, Barrel & 1.40 \\
\hline 19 & $2: 00$ & $8: 16$ & $10: 16$ & Full Dirt, 2 Barrels & 1.40 \\
\hline 20 & $2: 08$ & $8: 50$ & $10: 58$ & 1/2 Barrel, 2 Cement Rounds & 0.70 \\
\hline 21 & $2: 30$ & $8: 20$ & $10: 50$ & 1/2 Two Barrels, 3' Beam & 0.70 \\
\hline 22 & $1: 47$ & $8: 33$ & $10: 20$ & 1/4 Barrel, (1) 50' Cable & 0.35 \\
\hline 23 & $3: 18$ & $8: 33$ & $11: 51$ & 3/4 Paper \& Dirt & 1.05 \\
\hline 24 & $1: 27$ & $9: 15$ & $10: 42$ & $\begin{array}{l}\text { 3/4 Paper \& Dirt } 6 \times 6 \times 3^{\prime} \\
\text { Wood }\end{array}$ & 1.05 \\
\hline 25 & $1: 33$ & 9:03 & $10: 36$ & Full Paper \& Dirt, 3' Beam & 1.40 \\
\hline
\end{tabular}


Table H-4. TTV placement measurements at load station.

\begin{tabular}{|c|c|c|c|c|c|}
\hline \multicolumn{6}{|c|}{ TEST DATE: 9.28-94 } \\
\hline & \multicolumn{3}{|c|}{ FRONT MEASUREMENTS } & \multicolumn{2}{|c|}{ SIDE MEASUREMENTS } \\
\hline Cycle & $\begin{array}{l}\text { Ultrasonic } \\
\text { (inches) }\end{array}$ & $\begin{array}{l}\text { Manual } \\
\text { (inches) }\end{array}$ & $\begin{array}{l}\text { Difference } \\
\text { (inches) }\end{array}$ & $\begin{array}{l}\text { Manual } \\
\text { (inches) }\end{array}$ & $\begin{array}{c}\text { Difference } \\
\text { (inches) }\end{array}$ \\
\hline 1 & 91.2 & 94.0 & 0 & 28.0 & 0 \\
\hline 2 & 85.2 & 88.5 & 5.5 & 32.0 & 4.0 \\
\hline 3 & 94.0 & 86.0 & 8.0 & 39.0 & 11.0 \\
\hline 4 & 87.6 & 90.5 & 3.5 & $* 61.5$ & 0 \\
\hline 5 & 85.2 & 88.0 & 6.0 & 55.0 & 6.5 \\
\hline 6 & 87.6 & 90.5 & 3.5 & 43.5 & 18.0 \\
\hline 7 & 85.2 & 89.5 & 4.5 & 55.5 & 6.5 \\
\hline 8 & 84.0 & 87.5 & 6.5 & 59.5 & 2.0 \\
\hline 9 & 87.6 & 90.0 & 4.0 & 62.5 & 1.0 \\
\hline 10 & 87.6 & 86.5 & 7.5 & 66.5 & 5.0 \\
\hline 11 & 84.0 & 88.0 & 6.0 & 75.0 & 13.5 \\
\hline 12 & 86.4 & 90.5 & 3.5 & 70.5 & 9.0 \\
\hline 13 & 84.0 & 88.5 & 5.5 & 91.0 & 30.5 \\
\hline 14 & 79.2 & 84.0 & 10.0 & 108.5 & 47.0 \\
\hline 15 & 79.2 & 83.0 & 11.0 & 67.5 & 6.0 \\
\hline 16 & 80.4 & 84.0 & 10.0 & 69.5 & 8.0 \\
\hline 17 & 82.8 & 87.5 & 6.5 & 61.5 & 0 . \\
\hline 18 & 85.2 & 92.0 & 2.0 & 48.0 & 13.5 \\
\hline 19 & 88.8 & 96.0 & 2.0 & 58.0 & 3.5 \\
\hline $\begin{array}{l}\text { NOTE: } \\
\text { column i } \\
\text { measure } \\
\text { *Change }\end{array}$ & $\begin{array}{l}\text { le } 1 \text { Manual } N \\
\text { difference b } \\
t \text { at cycle } 1 . \\
\text { e Target -- Ne }\end{array}$ & $\begin{array}{l}\text { isurement w: } \\
\text { seen that cyc } \\
\text { Base Measur }\end{array}$ & $\begin{array}{l}\text { manual mea } \\
\text { me the } B\end{array}$ & $\begin{array}{l}\text { iasurement. } \\
\text { ent and the }\end{array}$ & $\begin{array}{l}\text { Difference } \\
\text { sual }\end{array}$ \\
\hline
\end{tabular}


Table H-5. TTV placement measurements at load station.

\begin{tabular}{|c|c|c|c|c|c|}
\hline \multicolumn{6}{|c|}{ TEST DATE: 9-29-94 } \\
\hline \multirow[b]{2}{*}{ Cycle } & \multicolumn{3}{|c|}{ FRONT MEASUREMENTS } & \multicolumn{2}{|c|}{ SIDE MEASUREMENTS } \\
\hline & $\begin{array}{l}\text { Ultrasonic } \\
\text { (inches) }\end{array}$ & $\begin{array}{l}\text { Manual } \\
\text { (inches) }\end{array}$ & $\begin{array}{l}\text { Difference } \\
\text { (inches) }\end{array}$ & $\begin{array}{l}\text { Manual } \\
\text { (inches) }\end{array}$ & $\begin{array}{c}\text { Difference } \\
\text { (inches) }\end{array}$ \\
\hline 1 & 118.8 & 125.5 & 0 & 64.0 & 0 \\
\hline 2 & 114.0 & 119.5 & 6.0 & 64.0 & 0 \\
\hline 3 & 115.2 & 122.0 & 3.5 & 64.0 & 0 \\
\hline 4 & 117.6 & 124.0 & 1.5 & 64.0 & 0 \\
\hline 5 & 120.0 & 127.0 & 1.5 & 65.5 & 1.5 \\
\hline 6 & 114.0 & 120.5 & 5.0 & 61.0 & 3.0 \\
\hline 7 & 117.6 & 124.0 & 1.5 & 68.0 & 4.0 \\
\hline 8 & 117.6 & 115.5 & 10.0 & 55.0 & 9.0 \\
\hline 9 & 114.0 & 110.0 & 15.5 & 53.0 & 10.0 \\
\hline 10 & 118.8 & 115.5 & 10.0 & 50.5 & 13.5 \\
\hline 11 & 120.0 & 114.5 & 11.0 & 57.5 & 6.5 \\
\hline 12 & 126.0 & 122.0 & 3.5 & 51.5 & 12.5 \\
\hline 13 & 121.2 & 118.5 & 7.0 & 57.0 & 7.0 \\
\hline 14 & 123.6 & 117.0 & 8.5 & 59.0 & 5.0 \\
\hline 15 & 116.4 & 123.5 & 2.0 & 45.5 & 18.5 \\
\hline 16 & 116.4 & 122.0 & 3.5 & 58.5 & 5.5 \\
\hline 17 & 122.4 & 128.5 & 3.0 & 53.0 & 11.0 \\
\hline 18 & 120.0 & 125.5 & 0 & 60.5 & 3.5 \\
\hline 19 & 120.0 & 125.5 & 0 & 55.5 & 8.5 \\
\hline 20 & 117.6 & 125.5 & 0 & 56.5 & 7.5 \\
\hline 21 & 118.8 & 125.5 & 0 & 54.5 & 9.5 \\
\hline 22 & 118.8 & 127.0 & 1.5 & 59.0 & 5.0 \\
\hline 23 & 123.6 & 129.0 & 3.5 & 51.0 & 13.0 \\
\hline 24 & 118.8 & 125.5 & 0 & 58.0 & 6.0 \\
\hline 25 & 118.8 & 125.5 & 0 & 54.5 & 9.5 \\
\hline
\end{tabular}


Table H-6. TTV placement measurements at unload station.

\begin{tabular}{||c|c|c|c||}
\hline \multicolumn{2}{||c|}{$\begin{array}{c}\text { Ultrasonic } \\
\text { (inches) }\end{array}$} & \multicolumn{2}{c|}{$\begin{array}{c}\text { Manual } \\
\text { (inches) }\end{array}$} \\
\hline 1 & 92.4 & 96.0 & $\begin{array}{c}\text { Difference } \\
\text { (inches) }\end{array}$ \\
\hline 2 & 100.8 & 105.0 & 0 \\
\hline 3 & 98.4 & 102.5 & 9.0 \\
\hline 4 & 98.4 & 96.5 & 6.5 \\
\hline 5 & 85.2 & 89.0 & 0.5 \\
\hline 6 & 85.2 & 89.5 & 7.0 \\
\hline 7 & 68.4 & $* 72.0$ & 6.5 \\
\hline 8 & 66.0 & 70.0 & 0 \\
\hline 9 & 66.0 & 69.5 & 2.0 \\
\hline 10 & 64.8 & 68.0 & 2.5 \\
\hline 11 & 63.7 & 67.25 & 4.0 \\
\hline 12 & 63.7 & 66.0 & 4.75 \\
\hline 13 & 61.2 & 65.0 & 6.0 \\
\hline 14 & 61.2 & 64.5 & 7.0 \\
\hline 15 & 62.4 & 66.5 & 7.5 \\
\hline 16 & 60.0 & 64.0 & 5.5 \\
\hline 17 & 64.8 & 68.5 & 8.0 \\
\hline 18 & 64.8 & 68.5 & 3.5 \\
\hline
\end{tabular}


Table H-7. TTV placement measurements at unload station.

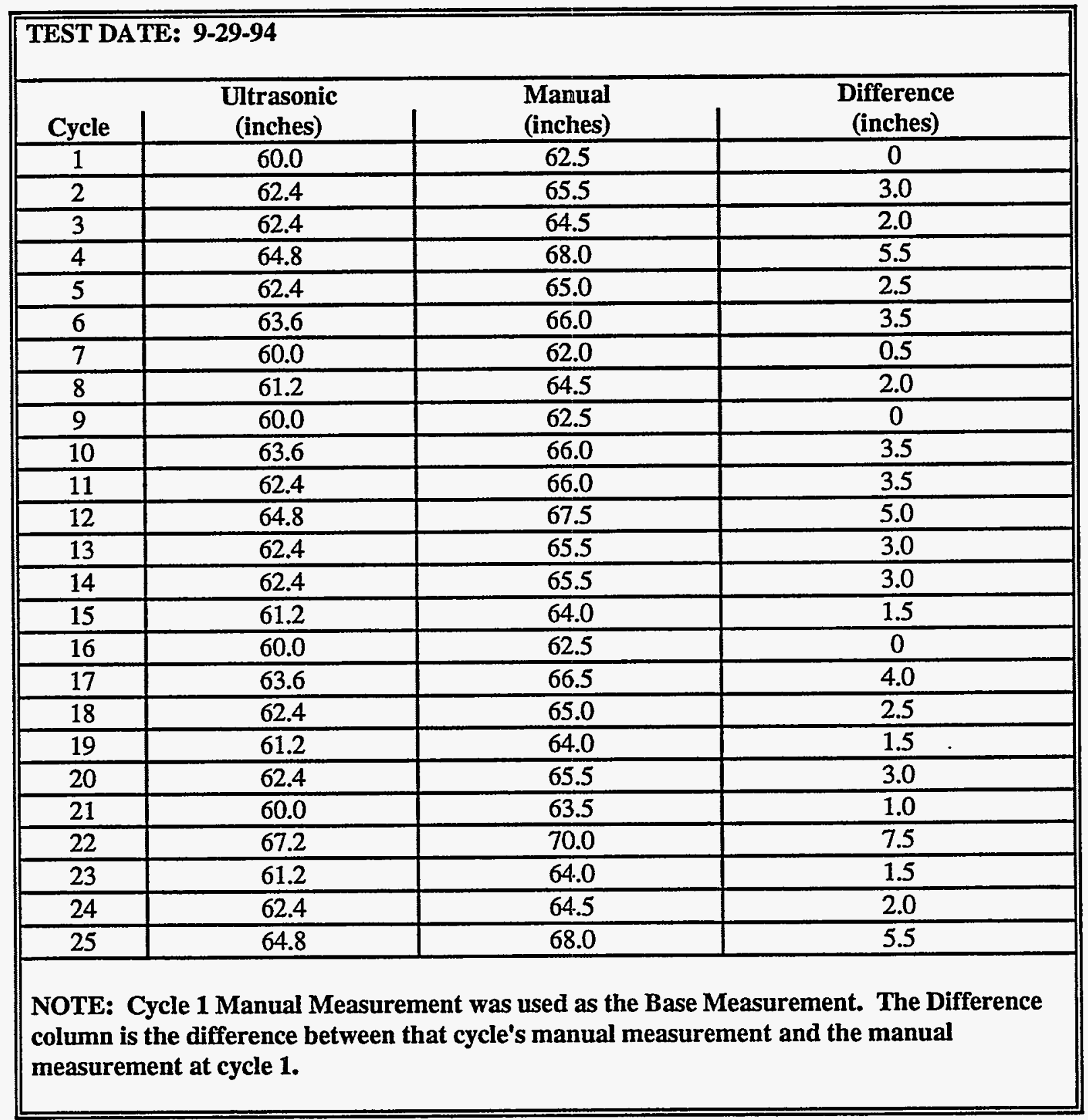


Table H-8. TTV maneuverability performance test data.

\begin{tabular}{|c|c|c|c|c|c|c|c|c|c|c|}
\hline \multirow{2}{*}{$\begin{array}{l}\text { Marker } \\
\text { Number }\end{array}$} & \multicolumn{10}{|c|}{ Performance Run } \\
\hline & $1^{* *}$ & $2^{* *}$ & $3^{* *}$ & 4 & 5 & 6 & 7 & 8 & 9 & 10 \\
\hline 1 & -0.5 & 0.0 & 0.0 & -0.5 & 1.0 & 2.5 & 2.0 & -4.0 & -12.0 & -2.0 \\
\hline 2 & 1.5 & 0.5 & 1.5 & 1.5 & 3.0 & 2.5 & 5.0 & 4.0 & 4.0 & -2.5 \\
\hline 3 & 7.0 & 4.5 & 4.0 & 5.0 & 3.5 & 3.5 & 11.5 & 8.0 & 9.0 & -1.5 \\
\hline 4 & 5.5 & 5.0 & 6.5 & 6.0 & 4.5 & 3.5 & 8.0 & 7.0 & 23.0 & 10.5 \\
\hline 5 & 0.5 & 3.0 & 2.0 & 4.0 & 4.0 & 4.0 & 3.5 & 8.5 & 20.0 & 7.5 \\
\hline 6 & 2.0 & 1.5 & 3.0 & 2.0 & 1.0 & 3.0 & 2.0 . & 4.0 & 13.0 & 1.5 \\
\hline 7 & 5.0 & 2.0 & 3.0 & 1.5 & 1.5 & 1.0 & 5.0 & 7.5 & 8.0 & 2.0 \\
\hline 8 & 8.0 & 7.5 & 6.0 & 4.0 & 0.0 & 3.0 & 8.0 & 9.0 & 11.0 & 1.0 \\
\hline 9 & $-1.0^{\circ}$ & -2.0 & -0.5 & -2.0 & -2.5 & -4.0 & -1.0 & 0.0 & -2.0 & -1.0 \\
\hline 10 & - & - & - & - & - & - & - & - & - & - \\
\hline Average & 3.1 & 2.4 & 2.8 & 2.4 & 1.8 & 2.1 & 4.9 & 4.9 & 8.2 & 1.7 \\
\hline Std. Deviation & 3.1 & 2.7 & 2.3 & 2.5 & 2.1 & 2.3 & 3.6 & 4.2 & 10.1 & 4.2 \\
\hline Time & - & $2: 45$ & $2: 49$ & $2: 55$ & $2: 50$ & $2: 53$ & $2: 20$ & $2: 22$ & $2: 54$ & $3: 11$ \\
\hline
\end{tabular}

${ }^{* *}$ Runs 1 through 3 were performed using Direct Track. All others used the Remote Track control mode.

The table shows the distance (in feet) from the desired path to the actual path of the TTV for each of the different operators. The time is how long it took the operator to guide the TTV through the course.

The Maneuverability Performance Test was conducted by having 5 people maneuver the TTV through a course. Performance run numbers 1 through 6 were performed by the demonstration TTV operator. Runs 7 through 10 were performed by novice operators who had never before operated the TTV.

The values in the table were measured by two people using a tape measure. They measured the distance from the centerline of the vehicle to the outside marker post on the course as the vehicle passed each marker post. The numbers in the table were computed from that data to show the distance by which the vehicle path differed from the intended path. 


\section{APPENDIX H CALCULATION DEFINITIONS}

The following cycles were not included in the average production rate (See Table H-2) calculations:

\begin{tabular}{rrl}
\hline Test date & Cycle & \multicolumn{1}{c}{ Comment } \\
\hline $9-27-94$ & 1 & Practice cycle \\
$9-28-94$ & 1 & Practice cycle \\
& 2 & Supervisory pack default delay \\
& 3 & Supervisory pack default delay \\
& 4 & Blown fuse delay \\
& 6 & Not timed \\
& 14 & Not timed \\
& 19 & Not timed \\
& 1 & Not timed \\
& 14 & Not timed \\
& 16 & Supervisory pack default delay \\
\hline
\end{tabular}

